\title{
The Memory Tesseract: Developing A Unified Framework for Modelling Memory and Cognition
}

by

Matthew A. Kelly

A thesis submitted to the Faculty of Graduate and Postdoctoral Affairs in partial fulfillment of the requirements for the degree of

Doctor of Philosophy

in

Cognitive Science

Carleton University

Ottawa, Ontario

(C) 2016, Matthew A. Kelly 


\section{Abstract}

Computational memory models can explain the behaviour of human memory in diverse experimental paradigms - whether it be recall or recognition, short-term or long-term retention, implicit or explicit learning. Simulation has led to parsimonious theories of memory, but at a cost of a profusion of competing models. As different models focus on different phenomena, there is no best model. However, the models share many characteristics, indicating wide agreement on the mathematics of how memory works in the brain.

On the basis of an analysis of computational memory models, we argue that these models can be understood in terms of a single neurally-plausible computational and theoretical framework. We present a proof of concept neural implementation, integration with the ACT-R cognitive architecture, and demonstrate model performance on procedural, declarative, episodic, and semantic learning tasks.

This research aims to advance cognitive psychology towards a single integrated, computational model of human memory that can account for human performance on diverse experimental tasks, that can be implemented at a neural level of detail, and can be scaled to modelling arbitrarily long-term learning. 


\section{Acknowledgements}

Thanks to Dr. Robert West, for his supervision, encouragement, and insight, and many conversations over hot chocolate in Rooster's. Thanks to my method rotation supervisors, Dr. Douglas Mewhort and Dr. Terrence Stewart, for their insight and advice. Thanks to Dr. Kam Kwok, for working with me on modelling the fan effect, and explaining the ACT-R fan effect model to me. Thanks also to Dr. Matthew Rutledge-Taylor for discussing his DSHM model with me and for collaborating with me on the journal publication of Chapter 8 . Thanks to my mom for her academic advice and my dad for his help with running simulations in FORTRAN. Thanks also to my sister, Sarah, for collaborating me with on writing projects completely unrelated to this thesis. Thanks to friends and fellow students of cognitive science Eric Iacono, for many esoteric late night conversations, Dr. Liz Christie, for hosting our weekly D\&D game, and Dr. Deirdre Kelly, for too many things to name. Finally, thanks to my parents, sister, and all of my friends for all of their support. 


\section{Table of Contents}

Abstract

Acknowledgements

Table of Contents iv

List of Tables $\quad$ ix

List of Figures $\quad$ x

List of Symbols $\quad$ xii

List of Computational Models $\quad$ xiii

List of Memory Models and Example Applications $\quad$ xv

Taxonomy of Computational Models of Human Memory $\quad$ xvi

$\begin{array}{ll}\text { Chapter } 1 \text { Introduction } & 1\end{array}$

1.1 Overview of Research Goals 1

1.2 Developing a Theoretical Framework for Vector-Based Memory Models 3

$\begin{array}{ll}1.3 \text { How is Memory Embedded in the Mind? } & 6\end{array}$

1.4 How is Memory Instantiated in Neurons?

1.5 Organization of Thesis 9

$\begin{array}{ll}1.6 \text { Prior Publication } & 15\end{array}$

Chapter 2 Vectors To Symbols: The Sub-Symbolic Level 16

2.1 Introduction 16

2.2 The Sub-Symbolic Level 18

2.3 n-Space and Similarity 19

2.4 Atomic versus Complex representations 20

2.5 Superposition (+) versus Binding (*) 21 
$\begin{array}{ll}2.7 \text { Permutation } & 24\end{array}$

Chapter 3 Addressing Criticisms of Connectionist and Symbolic Models 26

3.1 Addressing Criticism of Connectionism 26

$\begin{array}{ll}3.2 \text { Productivity Critique } & 27\end{array}$

3.3 Part-Whole Problem 28

3.4 Binding in Working v.s. Long-Term Memory 29

3.5 Vector Symbolic versus Symbolic 30

3.6 Best of Both Worlds? 31

3.7 Conclusions $\quad 32$

Chapter 4 Symbols to Cognition: The Symbolic Level 34

4.1 The Symbolic Level 34

4.2 Encoding and Storage 35

$\begin{array}{ll}4.3 \text { Retrieval } & 39\end{array}$

4.4 Conclusions 43

Chapter 5 The Memory Tesseract: Distributed MINERVA 46

5.1 Introduction 46

5.2 How does MINERVA work?

5.3 A comparison of memory models $\quad 54$

5.4 Composite vector models $\quad 55$

5.5 Matrix and tensor models $\quad 56$

5.6 Holographic vector models $\quad 58$

5.7 Multi-vector models $\quad 61$

5.8 MINERVA versus vector and matrix models 65 
$\begin{array}{ll}5.9 \text { Scalability of MINERVA } & 68\end{array}$

$\begin{array}{ll}5.10 \text { Similarity in MINERVA } & 70\end{array}$

5.11 MINERVA as a fourth order tensor $\quad 71$

5.12 Implications of the memory tesseract 78

5.13 Is the memory tesseract practical? $\quad 80$

5.14 Using holographic vectors rather than tensors 82

5.15 Neural implementation of the holographic model 87

$\begin{array}{ll}5.16 \text { Simulations } & 92\end{array}$

5.17 Approximating the Exemplar Production Model 96

$\begin{array}{ll}5.18 \text { Discussion } & 100\end{array}$

$\begin{array}{ll}5.19 \text { Conclusion } & 105\end{array}$

Chapter 6 Proof of Concept Neural Implementation of MINERVA 106

6.1 The Semantic Pointer Unified Architecture 106

6.2 Implementing MINERVA in NENGO 108

$\begin{array}{ll}\text { 6.3 Testing the MINERVA Model } & 108\end{array}$

$\begin{array}{lr}6.4 \text { Results } & 109\end{array}$

6.5 Recurrent MINERVA in NENGO 111

$\begin{array}{ll}\text { 6.6 Discussion } & 113\end{array}$

6.7 Holographic Approximation to MINERVA 115

$\begin{array}{ll}6.8 \text { Conclusions } & 116\end{array}$

$\begin{array}{lr}\text { Chapter } 7 \text { The Fan Effect and ACT-R } & 117\end{array}$

$\begin{array}{ll}7.1 \text { Introduction } & 117\end{array}$

$\begin{array}{ll}\text { 7.2 Holographic Models of Memory } & 119\end{array}$

$\begin{array}{llr}7.3 & \text { Fan Effect } & 120\end{array}$ 
7.4 ACT-R's Declarative Memory (DM)

$\begin{array}{ll}7.5 \text { Holographic Declarative Memory (HDM) } & 124\end{array}$

7.6 Memory Encoding and Recall with Slots 126

$\begin{array}{ll}7.7 \text { Vectors without Slots } & 129\end{array}$

7.8 Recognition with Holographic Declarative Memory 130

7.9 The HDM Model of the Fan Effect 130

7.10 Future Work and Applications of HDM 135

$\begin{array}{ll}7.11 \text { Conclusion } & 136\end{array}$

Chapter 8 Procedural Learning and Decision Making 138

$\begin{array}{ll}8.1 \text { Introduction } & 138\end{array}$

$\begin{array}{ll}8.2 \text { Experiment } & 140\end{array}$

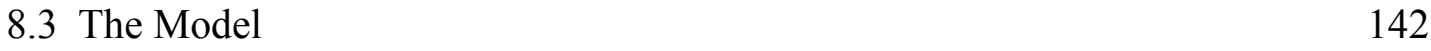

8.4 Comparing the model to human performance 144

8.5 Representing positive and negative feedback 146

8.6 Initializing memory to a state of optimism 147

8.7 Updating memory using open n-grams 149

$\begin{array}{ll}8.8 \text { Probing memory } & 150\end{array}$

$\begin{array}{ll}8.9 \text { Discussion } & 153\end{array}$

Chapter 9 Memory for Abstract Associations $\quad 155$

$\begin{array}{ll}9.1 \text { Overview } & 155\end{array}$

9.2 Orders of Association 157

$\begin{array}{ll}\text { 9.3 Distributional Models of Semantics } & 160\end{array}$

$\begin{array}{ll}\text { 9.4 The BEAGLE Model } & 162\end{array}$

9.5 Hierarchical Holographic Model 164 
9.6 Simulation 1: Results

$\begin{array}{ll}9.6 \text { Simulation 1: Discussion } & 167\end{array}$

$\begin{array}{ll}9.7 \text { Simulation } 2 & 168\end{array}$

$\begin{array}{ll}9.8 \text { Simulation 3: Method } & 169\end{array}$

$\begin{array}{ll}9.9 \text { Simulation 3: Results } & 171\end{array}$

$\begin{array}{ll}\text { 9.10 Simulation 3: Discussion } & 176\end{array}$

$\begin{array}{ll}9.11 \text { Simulation } 4 & 176\end{array}$

$\begin{array}{ll}9.12 \text { General Discussion } & 184\end{array}$

$\begin{array}{ll}9.13 \text { Conclusion } & 187\end{array}$

Chapter 10 Memory Model Framework $\quad 189$

$\begin{array}{lll}10.1 \text { Towards Unification } & 189\end{array}$

10.2 Hetero-Associative versus Auto-Associative 191

10.3 Errorless versus Error-driven Learning 199

10.4 Concept-based versus instance-based models and the placeholder vector $\quad 200$

10.5 HDM, MINERVA and the Fan Effect 203

10.6 Generating Response Time Predictions 210

10.7 Framework For Computational Models of Human Memory 213

$\begin{array}{ll}10.8 \text { Proposal for Future Models of Memory } & 220\end{array}$

$\begin{array}{ll}10.9 \text { Conclusion } & 222\end{array}$

$\begin{array}{lr}\text { Chapter } 11 \text { Conclusion } & 223\end{array}$

$\begin{array}{ll}\text { 11.1 Overview of Contributions } & 227\end{array}$

$\begin{array}{ll}\text { References } & 233\end{array}$ 


\section{List of Tables}

$2.1 \quad$ Comparison of VSAs and tensor representations 18

8.1 Stimulus and choices for model of the Walsh and Anderson's (2011) task 143

$\begin{array}{lll}9.1 & \text { Artificial data set for Simulation } 1 & 158\end{array}$

9.2 Cosine similarities between pairs of memory vectors in Simulation 1

9.3 Animal facts for two of the animals in Simulation 3 171

9.4 Top 10 word-pairs with the highest cosines on each layer in Simulation $4 \quad 177$

9.5 Cosine means and standard deviations for each layer in Simulation $4 \quad 178$

10.1 Comparison across memory models of matching strengths in recognition 192

10.2 Equations for analytic matching strengths of models of the fan effect task 207 


\section{List of Figures}

5.1 MINERVA 2 represented as a localist neural network $\quad 53$

5.2 Iterative retrieval from five memory models $\quad 82$

5.3 Iterative retrieval from the holographic approximation to the tesseract 86

5.4 A biologically plausible neural network for computing circular convolution $\quad 88$

5.5 A fully distributed biologically plausible neural model of MINERVA $2 \quad 90$

5.6 A comparison of the echoes from four memory models 95

$5.7 \%$ correct on test items by the holographic approximation to the EPM 98

5.8 Mean ranking of test items by the holographic approximation to the EPM 99

6.1 Echo from MINERVA with an exponent of $1 \quad 110$

6.2 Echo from MINERVA with an exponent of 2 111

6.3 Echo from MINERVA with an exponent of 3 111

6.4 Direct and neural recurrent MINERVA models in NENGO 112

6.5 Echo from the recurrent MINERVA model with an exponent of $3 \quad 113$

6.6 Echo from the recurrent MINERVA model with an exponent of 1

7.1 Real versus ACT-R DM reaction times for the fan effect 124

7.2 Real versus HDM reaction times for the fan effect 131

7.3 HDM vectors for the fan effect represented as points on a sphere 132

8.1 States and transition probabilities from the Walsh and Anderson's (2011) task 141

8.2 Response accuracy for human participants from Walsh and Anderson (2011) 145

8.3 Response accuracy for the HDM model of Walsh and Anderson's (2011) task 145

9.1 Cosine distances between memory vectors for Simulation $1 \quad 166$

9.2 Cosine distances between memory vectors for Simulation $2 \quad 169$

9.3 Cosine distances between memory vectors for Simulation $3 \quad 172$ 
9.4 Cosine similarities between sample vector pairs across layers for Simulation $3 \quad 173$

9.5 Cosine similarities between sample vector pairs across layers for Simulation $3 \quad 174$

9.6 Cosine distances for target animals and near neighbours in Simulation $3 \quad 175$

9.7 Cosine similarities between word pairs across layers in Simulation 4

9.8 Effect of each layer of the model in Simulation 4 on selected word pairs 181

9.9 Cosine distances for our and your and near neighbours in Simulation $4 \quad 182$

9.10 Cosine distances for back and up and near neighbours in Simulation 4

10.1 Simulated matching strengths for models of the fan effect task 204

10.2 Analytic matching strengths for models of the fan effect task 208 


\section{List of Symbols}

$n \quad$ Dimensionality of a vector

$m \quad$ Number of traces stored in memory

$p \quad$ Number of memory vectors in a Levy and Gayler (2009) network

- Dot product

- Element-wise multiplication

| Concatenation of vectors

* Circular convolution

\# Circular correlation

$\otimes \quad$ Tensor product

$\boldsymbol{\delta} \quad$ Identity vector for circular convolution: $\boldsymbol{\delta}=[1,0,0,0 \ldots]$

$\mathbf{a}^{\mathrm{T}} \quad$ Transpose of vector $\mathbf{a}$

$a_{i} \quad$ The $i$ th element of vector $\mathbf{a}$

Ф Placeholder vector, a random vector that represents the item in question

P Permutation matrix

$\mathbf{e}_{\text {item }}$ environment vector representing the percept of the item item

$\mathbf{m}_{\text {item }}$ memory vector representing all known about item

$\mathbf{q}_{\text {?bc }} \quad$ cue vector that asks "what came before the items $b$ and $c$ in this sequence?"

$\Delta$ Delta, change in. 


\title{
List of Computational Models
}

\author{
Acronym Model name (citation) \\ ACT-R Adaptive Control of Thought - Rational (Anderson \& Lebiere, 1999) \\ ACT-R DM $\quad$ ACT-R Declarative Memory module (Anderson \& Lebiere, 1999) \\ BEAGLE Bound Encoding of the Aggregate Language Environment (Jones \& Mewhort, 2007) \\ Binary spatter codes, a type of VSA (Kanerva, 1996) \\ CHARM Composite Holographic Associative Recall Model (Eich, 1982) \\ DSHM Dynamically Structured Holographic Memory (Rutledge-Taylor et al., 2014) \\ EPM Exemplar Production Model (Johns et al., 2016) \\ Franklin and Mewhort (2015) holographic vector model \\ GCM Generalized Context Model (Nosofsky, 1986, 1991) \\ HDM Holographic Declarative Memory (Chapter 7) \\ HEM Holographic Exemplar Model (Jamieson \& Mewhort, 2011) \\ HHM Hierarchical Holographic Model (Chapter 9) \\ HRR Holographic Reduced Representation, a type of VSA (Plate, 1995) \\ HyGene Hypothesis Generation model, a variant of MINERVA-DM (Thomas et al., 2008) \\ IRM Iterative Resonance Model (Mewhort \& Johns, 2005) \\ Levy and Gayler (2009) lateral inhibition network \\ many-to-many Class of memory models, stores many experiences across many vectors (Chapter 4) \\ many-to-one Composite/distributed storage models, many experiences in one tensor (Chapter 4) \\ MAP Multiply-add-permute coding (Gayler, 2003) \\ Matched Filter Model (Anderson, 1973; Murdock, 1989) \\ Matrix Matrix Model and 3D Matrix Model (Humphreys et al., 1989a, 1989b) \\ MINERVA 2 MINERVA 2, a model of episodic and semantic memory (Hintzman, 1986) \\ MINERVA-AL MINERVA - Associative Learning (Jamieson et al., 2012) \\ MINERVA-DM MINERVA - Decision Making (Dougherty et al., 1999) \\ NEF Neural Engineering Framework, a theory of neuro-computation (Eliasmith, 2013)
}




\section{Acronym Model name (citation)}

NENGO Neural Engineering Objects, platform for NEF models (Eliasmith, 2013)

one-to-one $\quad$ Class of memory models, stores one experience per one vector (Chapter 4)

- $\quad$ Osth and Dennis (2015) matrix model

Quantum Quantum models of human probability judgement (Busemeyer et al., 2011)

REM Retrieving Effectively from Memory model (Shiffrin \& Steyvers, 1997)

RSS Recognition through Semantic Synchronization (Johns et al., 2012)

SAM Search of Associative Memory (Gillund \& Shiffrin, 1984)

Soar The State, Operator, And Result cognitive architecture (Laird, 2012)

SOB \& C-SOB (Context) Serial-Order-in-a-Box model (Farrell \& Lewandowsky, 2002)

SPAUN Semantic Pointer Architecture Unified Network (Eliasmith et al., 2012)

TCM Temporal Context Model (Howard \& Kahana, 2002)

Tensor Tensor product representation (Smolensky 1990)

Tesseract Memory tesseract, an auto-associative fourth order tensor memory (Chapter 5)

TODAM Theory of Distributed Associative Memory (Murdock, 1993)

VSA Vector-symbolic architecture (Gayler, 2003; see Kelly et al., 2013 for review) 


\title{
List of Memory Models and Example Applications
}

\author{
Model \\ ACT-R DM \\ BEAGLE \\ CHARM \\ DSHM \\ EPM \\ Franklin \& Mewhort \\ GCM \\ HEM \\ HyGene \\ IRM \\ Levy \& Gayler \\ Matched Filter \\ Matrix \\ MINERVA 2 \\ MINERVA-AL \\ MINERVA-DM \\ REM \\ RSS \\ SAM \\ SOB \& C-SOB \\ TCM \\ TODAM

\section{Applications} \\ fan effect task, recency, frequency, cued recall, recognition, response times \\ distributional semantics \\ cued recall, prototype abstraction \\ fan effect task, problem size effect, rock-paper-scissors \\ syntactic ordering of words into sentences \\ primacy effect, recency effect, free recall, order of recall \\ category learning \\ artificial grammar task \\ hypothesis making, judgement of likelihood, and decision making \\ response time prediction, rejection of the foil \\ analogical mapping \\ recognition \\ cued recall, recognition, amnesia \\ category learning, prototype abstraction, recognition, cued recall \\ associative learning \\ likelihood judgements \\ list strength effects, recognition \\ list strength effects, mirror effect, recognition, false recognition \\ recognition, cued recall, part list cueing \\ response time prediction, serial recall, primacy effect, recency effect \\ recency effect, temporal contiguity effects \\ recency effect, recognition, cued recall, frequency effects
}


Taxonomy of Computational Models of Human Memory

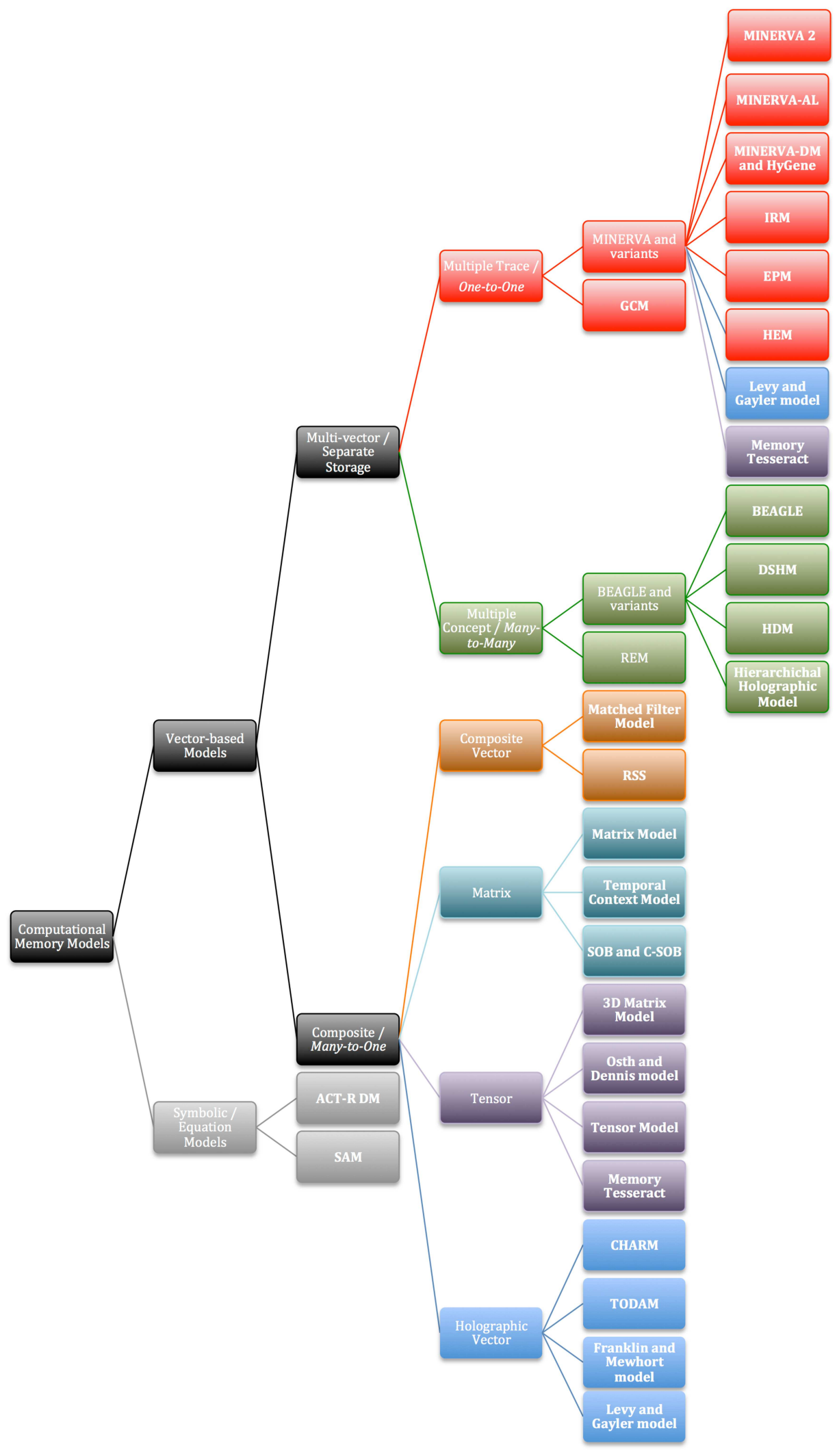

xvi 


\section{Chapter 1 Introduction}

\subsection{Overview of Research Goals}

Experimental work has suggested to researchers that humans have many different kinds of memory, such as episodic and semantic, short-term and long-term, and implicit and explicit. However, by developing computational models of memory, researchers have found that many of the behavioural phenomena that had been taken as evidence for disparate memory systems can be accounted for by unified memory systems (e.g., Anderson \& Lebiere, 1998; Franklin \& Mewhort, 2015; Hintzman, 1984; Humphreys, Bain, \& Pike, 1989a; Jamieson \& Mewhort, 2011; Raaijmakers \& Shiffron, 1981). The use of computational models has lead to more parsimonious and unified theories, but it replaces a profusion of different kinds of memory with a profusion of competing models. As different models tend to focus on different subsets of memory phenomena, there is no best model.

Our research has two goals:

(1) To work towards a unified theory of memory through careful analysis of the competing memory models in the literature. A unified model of memory should be able to be implemented at a neural level of detail, scaled to modelling arbitrarily long-term learning, and account for human performance on diverse experimental tasks.

(2) Through integration of a powerful model of memory with a cognitive architecture, work towards a unified theory of cognition. 
Achieving these goals in full is beyond the scope of a mere Ph.D. thesis, but we hope to help provide a foundation upon which a unified theory of memory can be achieved, and we demonstrate integration of our memory model with a cognitive architecture.

One could argue that the profusion of different models in the literature is indicative of a healthy amount of experimentation, as the discipline is too young to say in advance what ideas will ultimately be the right ones. Nevertheless, we ask, is research in human memory leading us toward a unified theory of memory? Or is it leading us toward a patchwork quilt of different theories, and a particularly poor quilt at that, as the patches haven't even been sewn together? In this thesis, we explore the unification of different models and theories of memory. By unification, we mean the task of answering the following questions:

(1) To what extent do differences in theoretical description and computational implementation reflect actual differences in behavioural prediction? Models that have been understood as distinct may be different ways of presenting the same theoretical assumptions.

(2) Do the different memory models share common theoretical, mathematical, and computational assumptions about human memory? If so, these assumptions form a core theoretical framework that characterizes current memory theory.

(3) If we can characterize a common framework of assumptions across models of memory, can we also characterize a set of parameters within that framework that describes the space of exploration for current and future models of memory?

(4) When different models account for different sets of behavioural phenomena, is this evidence that there is in fact separate memory systems (e.g., declarative and procedural, or 
episodic and semantic), or is it evidence that both models are incorrect and that the correct model would be able to account for both sets of behavioural phenomena?

(5) Can a computational model of memory be developed that accounts for all basic human memory phenomena?

Answering question (5), that is, creating a unified model of human memory is beyond the scope of this thesis. Indeed, it is beyond the scope of what can be undertaken by any one researcher. To ever achieve a unified theory of memory, we need researchers working in a common theoretical framework. Does such a framework exist implicitly in the memory models in the literature? In this thesis we explore the answers to questions (1), (2), and (3) to lay a foundation for future research that works towards answering (4) and (5).

\subsection{Developing a Theoretical Framework for Vector-Based Memory Models}

The various memory models have a great deal in common, indicating wide agreement on how memory works. Through mathematical analysis, we show that many of the memory models in the literature can be understood as sharing a common mathematical framework. We demonstrate that this framework is neurally plausible and scalable to long-term learning.

The memory models in this framework include the MINERVA 2 (Hintzman, 1984) model and variants (e.g., Jamieson, Crump, \& Hannah, 2012; Jamieson \& Mewhort, 2011; Kwantes, 2005; Thomas et al., 2008), which together account for a wide swath of episodic, semantic, declarative, and procedural memory phenomena, as well as holographic models of short and long term memory (e.g., Murdock, 1993; Franklin \& Mewhort, 2015), the BEAGLE model of distributional semantics (Jones \& Mewhort, 2007), the DSHM model of declarative memory 
(Rutledge-Taylor, Kelly, West, \& Pyke, 2014), SAM (Raaijmakers \& Shiffrin, 1981; Gillund \& Shiffrin, 1984), the Generalized Context Model of category learning (Nosofsky, 1986, 1991), composite vector models (e.g., Anderson, 1973; Johns, Jones, \& Mewhort, 2012; Murdock, 1989), matrix models (e.g., Farrell \& Lewandowsky, 2002; Humphreys, Pike, Bain, \& Tehan, 1989b; Howard \& Kahana, 2002; Lewandowsky \& Farrell, 2008), tensor models (e.g., Humphreys, Bain, \& Pike, 1989a; Osth and Dennis, 2015; Smolensky, 1990), and ACT-R's model of declarative memory (ACT-R DM; Anderson \& Lebiere, 1998). In our analysis, we have chosen to focus on vector-based models of memory, though we include two models of memory that are not vector-based: ACT-R DM and SAM.

We use the MINERVA 2 model (Hintzman, 1984) as a starting point for developing our theoretical framework. We choose MINERVA 2 because it is a model that can capture a wide variety of human memory phenomena across differing experimental paradigms and as such seems a good candidate for a basis for theoretical unification. While there are many experimental phenomena that MINERVA 2 cannot account for, our interest is not so much in MINERVA 2 per se, but in the broader class of models based on MINERVA 2. We refer to this broader class of models as MINERVA models and use MINERVA 2 to refer specifically to the model proposed by Hintzman (1984). While MINERVA 2 has many limitations, the MINERVA framework as a whole has proved a fruitful research paradigm. In particular, MINERVA-AL (Jamieson et al., 2012), which extends MINERVA 2 to model associative learning, and HEM (Jamieson \& Mewhort, 2011), which integrates MINERVA with a scheme of knowledge representation called holographic vectors, are proving to be promising directions for the MINERVA framework. 
We prove that MINERVA is equivalent to a distributed memory system, namely, a fourth order tensor, or "memory tesseract" (Chapter 5). In geometry, a tesseract is a four dimensional analog to a cube, just as the cube is a three dimensional analog to a square. We use the term memory tesseract for a memory system that stores all knowledge distributed across a data structure with four equal dimensions. We additionally suggest an alternative implementation of the memory tesseract as a holographic lateral inhibition network (Levy \& Gayler, 2009). This alternative implementation is less computationally expensive, though only approximately equivalent to MINERVA due to lossy compression.

The equivalence between MINERVA and the memory tesseract provides new insight into the relationship between MINERVA and other memory models. On the basis of this equivalence, we argue that models such as BEAGLE (Jones \& Mewhort, 2007), DSHM (Rutledge-Taylor et al., 2014), and ACT-R's DM (Anderson \& Lebiere, 1998), can be understood as higher-level descriptions of the memory tesseract, and that other memory models, such as TODAM (Murdock, 1993) or the Matrix Model (Humphreys, Bain, \& Pike, 1989a; Humphreys, Pike, Bain, \& Tehan, 1989b), are only minor variations on the tesseract model.

To aid the reader, at the beginning of this thesis, we provide two lists of models and a taxonomy (pp. xiii-xvi). List of Computational Models (pp. xiii-xiv) is a list of all of the computational models that we will discuss, which includes each model's acronym, name, and citation. Not all of the models listed are memory models. For example, ACT-R is a cognitive architecture, a model of the mind as a whole, and HRRs are a tool for representing knowledge in cognitive models. 
List of Memory Models and Example Applications (p. xv) is a list of computational memory models annotated with the kinds of behavioural data and experimental tasks accounted for by each model. We provide this list to give the reader a sense of the applications of each memory model. The list of applications is not comprehensive and is for illustrative purposes only. As we will discuss, there are strong mathematical similarities between these models. As such, many of these models could be adapted to applications listed under other models.

Lastly, the Taxonomy of Computational Models of Human Memory (p. xvi) illustrates how the models we discuss are related to each other. The taxonomy is not comprehensive: it is limited to the models discussed in this thesis. The taxonomy includes many of the vector-based memory models in the literature, but only two equation models: SAM and ACT-R DM. Vectorbased memory models are the focus of this thesis. However, we include the SAM model (Raaijmakers \& Shiffrin, 1981; Gillund \& Shiffrin, 1984) in our discussion because SAM's equations can be understood as a description of a vector-based memory model, as noted by Humphreys et al. (1989b). We include ACT-R DM because we explore integration of a vectorbased model of memory into the ACT-R cognitive architecture (Chapter 7).

\subsection{How is Memory Embedded in the Mind?}

Newell (1973) famously argued that you can't play twenty questions with nature and win: that the accumulation of binary answers to carefully asked experimental questions, by either rejecting or accepting the null hypothesis, is not in itself leading psychology to a theory of the mind. Newell's suggested solution is to attempt to create a computational model of cognition in its 
entirety. To develop a unified theory of cognition we need a computational model sufficiently flexible and powerful to account for how minds perform a wide variety of tasks.

Newell is not, by any means, alone in this view. Other prominent cognitive scientists such as Brooks (1991) and Pylyshyn (1978) have also made the case that the divide and conquer approach has been working against psychologists. Newell, Pylyshyn, and Brooks' observations emphasize the importance of striving towards theoretical unification, not just in modelling memory, but in modelling cognition as a whole. In order to understand the parts, we must have an idea how they fit into a comprehensive whole. To understand memory, we need to understand how memory is embedded in the mind.

To embed a theory of memory into a theory of the mind as a whole, we integrate a memory model from our theoretical framework into the ACT-R cognitive architecture. ACT-R (Adaptive Control of Thought - Rational; Anderson \& Lebiere, 1998) is a widely used system aimed at the goal of providing a unified theory of cognition. ACT-R serves as a sort of scripting language with built-in cognitive constraints that allows it to model human behaviour, specifically, to produce accuracy and timing predictions. To do this, the ACT-R Declarative Memory (DM) system is integrated into a larger architecture that allows the entire process of a task to be modelled, as opposed to only the memory storage and retrieval portions. However, the ACT-R DM system is limited due to its symbolic architecture, which uses words or symbols to represent ideas and stimuli.

We substitute ACT-R's memory system with a new model based on the BEAGLE (Jones \& Mewhort, 2007) model of distributional semantics and the DSHM (Rutledge-Taylor et al., 2014) memory model (Chapter 7). This new model, Holographic Declarative Memory (HDM), 
replaces the symbols of ACT-R with holographic vectors, with the aim of adding three new capabilities to ACT-R: (1) long-term learning and inference from a large knowledge base, (2) better partial-matching of situations to procedures and the ability to represent ambiguous or noisy stimuli typical of real-world environments, and (3) the ability to address questions of how memory may be realized in the brain. Reciprocally, situating our memory model within a broader theory of cognition, such as ACT-R, confers advantages for creating cognitive models using our memory model.

\subsection{How is Memory Instantiated in Neurons?}

A unified model of memory should be able to be implemented at a neural level of detail. Implementing a model of memory at a neural level of detail could allow the model to make detailed biological predictions and account for the influence of quirks of neuron behaviour on the memory system (e.g., in cases of neural dysfunction, such as Alzheimer's disease). At a minimum, a neural implementation demonstrates that the model can be realized in the human brain, and as such, is not an impossible theory of human memory.

ACT-R provides a description of mental processes and brain areas associated with them, but does not address the question of how those mental processes could be carried out at the neural level. Holographic models are easy to implement in realistic spiking neuron models and thus can address questions of neural implementation. Using the Neural Engineering Framework (Eliasmith, 2013), we present a proof of concept neural implementation of MINERVA as a holographic network (Chapter 5) and as the memory tesseract (Chapter 6). We demonstrate that MINERVA can mimic the behaviour of HDM (Chapters 5 and 10). Thus, HDM can be 
understood as a higher-level description of MINERVA. Because MINERVA can be realized in neurons (Chapters 5 and 6), and HDM can be realized by MINERVA (Chapter 5 and 10), and HDM can be substituted for ACT-R DM (Chapter 7), we thereby address the question of neural implementation of memory for MINERVA, HDM, ACT-R, and other models of memory in our theoretical framework.

\subsection{Organization of Thesis}

In the following chapters, we first provide an introduction to the linear algebra of vectorsymbolic architectures (Chapter 2) and our reasons for using vector-symbolic architectures (Chapter 3). We then survey the vector-based memory modelling literature (Chapters 4 and 5), present novel theoretical analysis and mathematical proofs relating to that literature (Chapter 5), then move on to work that uses that theoretical analysis to build neural models of memory (Chapters 5 and 6) and to model human performance on diverse tasks (Chapters 7 to 9). Finally, on the basis of further theoretical analysis of the memory models in the literature, we define a framework to guide development of future models of human memory (Chapter 10).

Chapter 2, Vectors to symbols: The sub-symbolic level is an introduction to the mathematics of vector symbolic architectures and a survey of the different vector symbolic architectures in the literature. Vector Symbolic Architectures (VSAs) are a set of techniques for instantiating and manipulating symbolic structures in distributed representations. These symbolic structures can be used to represent concepts and percepts in a cognitive model. VSAs have both a sub-symbolic level, at which they are understood in terms of linear algebra, and a symbolic level, at which they are understood in terms of manipulations of symbols that stand for concepts. 
Decisions need to be made about the model at both of these levels. In Chapter 2, we discuss the sub-symbolic, that is to say, mathematical decisions, and provide a brief overview of the different types of VSA in the literature. For our cognitive models, we adopt Holographic Reduced Representations (HRRs), a type of vector symbolic architecture formalized by Plate (1995).

In Chapter 3, Addressing criticisms of connectionist and symbolic models, we present our motivation for deciding to use VSAs for cognitive modelling. Using VSAs addresses a number of criticisms of traditional, non-recurrent neural networks, and traditional, purely symbolic modelling.

In Chapter 4, Symbols to cognition: The symbolic level, we discuss the decisions made at the symbolic level when using VSAs. We review a number of vector-based computational cognitive models of memory in the literature and classify the models into three types according to their structural differences: many-to-one, one-to-one, and many-to-many memory models.

In many-to-one models, all memories are distributed across a shared set of units. Many experiences are all summed into one composite store, a data structure that takes the form of a vector, matrix, or higher order tensor (such as the tesseract). Many-to-one models are known as distributed memory models, distributed storage models, or composite storage models in the literature.

In one-to-one models, each individual experience is stored as as a separate vector. There is a one-to-one relationship between the number of vectors in memory and the number of experiences stored in memory. These models are referred to as multiple-trace models in the literature, where a trace is a single memory, a record of a particular experience. MINERVA is an example of a one-to-one model. 
In many-to-many models, many experiences are stored across many vectors. Each concept or item in memory has a vector and the vector stores the relationships of that concept to all other concepts. By analogy to multiple-trace models, one can think of these as multiple-concept models. Just as a multiple-trace / one-to-one model has one vector per trace, these models have one vector per concept. BEAGLE (Jones \& Mewhort, 2007) and variants, such as DSHM (Rutledge-Taylor et al., 2014), the HDM model (Chapter 7), and Hierarchical Holographic Model (Chapter 9) are many-to-many models.

Collectively, many-to-many and one-to-one constitute what we refer to as multi-vector models, and what is referred to in the literature as separate storage models. The taxonomy of computational models of human memory presented at the beginning of this thesis illustrates the relationship between these models.

In Chapter 5, The memory tesseract: Distributed MINERVA, we present a formal proof of equivalence between the MINERVA 2 memory model (Hintzman, 1988) and an auto-associative fourth-order tensor memory, or memory tesseract. This proof shows that the many-to-one and one-to-one classes of model identified in Chapter 4 are not, in fact, structurally distinct from each other. We further prove that MINERVA 2 is approximately equivalent to the memory tesseract compressed into a holographic vector. This proof has three implications:

(1) The relationship between MINERVA-based models of memory and other kinds of memory models is clarified, moving us toward theoretical unification. This work also suggests that MINERVA and the memory tesseract may be suitable as a basis for all memory modelling. (2) MINERVA, implemented as a distributed system such as the memory tesseract, is scalable to arbitrarily long-term learning. 
(3) MINERVA can be plausibly implemented in neurons.

On the basis of this proof, we present a neural model of MINERVA implemented as the holographic vector approximation of the memory tesseract.

In Chapter 6, Proof of concept neural implementation of MINERVA, we present a proof of concept model of memory retrieval in MINERVA implemented as a recurrent, spiking neural network. The network is a realistic simulation of neurons built using the Neural Engineering Framework (NEF; Eliasmith, 2013). Unlike the model in Chapter 5, this model can be understood as a neural implementation of the memory tesseract as a tensor, rather than the memory tesseract as a holographic vector.

In Chapter 7, The fan effect and ACT-R, we integrate a vector-symbolic memory model with the ACT-R cognitive architecture (Anderson \& Lebiere, 1998). We argue that the vectorsymbolic memory model confers a number of advantages over the standard symbolic model of declarative memory used by ACT-R. Reciprocally, situating our memory model within a broader theory of cognition such as ACT-R confers advantages for creating cognitive models using our memory model. The memory model introduced in this chapter is Holographic Declarative Memory (HDM), which belongs to the many-to-many class of memory models discussed in Chapter 4. Notably, HDM is not a MINERVA-variant or memory tesseract model. Rather, HDM is a variant of the BEAGLE and DSHM models. However, as we demonstrate in Chapters 5 and 10 , it is possible to re-implement a many-to-many memory model in a MINERVA or memory tesseract architecture. In light of these demonstrations, we consider HDM an instantiation of the broader theoretical framework outlined in Chapter 5. 
Chapter 7 demonstrates performance of HDM on a recognition memory task, the fan effect (Anderson, 1974). According to Anderson's theory (1991), the availability of a memory in a given situation is an estimate of the probability of that memory being useful in that situation. Response times in the fan effect task seem to vary in accordance with that probability. We present an analysis of the behaviour of HDM, showing that an estimate of the probability that a memory is relevant to the current context arises naturally from the geometry of the vector space. This analysis has implications for HDM beyond the particulars of the fan effect task. Because the vector algebra of HDM estimates probability, HDM can be understood as approximating the behaviour of ACT-R DM and, more generally, Bayesian models. This also shows that HDM is closely related to the quantum probability model of human probability judgements (Busemeyer, Pothos, Franco, \& Trueblood, 2011), which also uses vector algebra to calculate probabilities. In Chapter 8, Procedural Learning and Decision Making, we use HDM to model human performance on a sequential decision-making task. ACT-R has two memory systems: a declarative memory, which stores knowledge of the probabilities of events, and a procedural memory, which stores knowledge of the utility of actions. In the previous chapter, we demonstrated that HDM can serve as an effective declarative memory. In Chapter 8, we show that HDM can also serve as an effective substitute for procedural memory and simulate human decision making. HDM's ability to model both declarative and procedural memory, as well as the ability of related models in the literature to model both rapid short-term learning (RutledgeTaylor et al., 2014) and long-term learning of large quantities of knowledge (Jones \& Mewhort 2007; Rutledge-Taylor et al, 2008), provide converging evidence of the suitability of HDM-like models as a versatile basis for modelling human memory. 
In Chapter 9, Memory for Abstract Associations, we ask, what happens if we aggregate across the units of a BEAGLE model to create new units? And then aggregate across those units to create new units? And so on? We show that a hierarchy of BEAGLE / HDM models, which we refer to as the Hierarchical Holographic Model, can detect higher-order associations between concepts. We demonstrate performance of the Hierarchical Holographic Model on simple artificial data sets and a natural language corpus consisting of a collection of children's novels. While the BEAGLE model is already capable of capturing a great deal of the semantic and syntactic relationships between words, by using the representations developed by one model and feeding them into the next model in the hierarchy, we show that we can bootstrap learning, allowing the model to detect more subtle syntactic and semantic relationships at higher layers. The work presented in Chapter 9 is a proof of concept of the utility of orders of association as a theoretical construct and the Hierarchical Holographic Model as an extension of the BEAGLE and HDM models.

In Chapter 10, Memory Model Framework, we build further on the comparative theoretical analysis of memory models presented in Chapters 4, 5, and 7 for the sake of furthering theoretical unification. We present a framework for computational memory models. This framework consists of common assumptions shared widely across memory models, a set of practical implementation decisions at symbolic and sub-symbolic levels of description, as well as important theoretical decisions in areas where consensus has yet to be reached. This framework is both descriptive and proscriptive: we intend to both describe the state of the literature and outline what we believe is the space of possibilities that will be most fruitful in the development of future memory models. In particular, we discuss the relationship between the MINERVA class 
and BEAGLE class of memory models in more detail, as these two classes of model have been the focus of this thesis. As we discuss, the three classes of model identified in Chapter 4 can be understood as multiple realizations of what is essentially the same architecture, allowing for theoretical unification, as well as a path to neural implementation for high-level memory models such as HDM.

Ultimately, we aim to provide a sketch of a foundation for the development of a unified computational model and theory of human memory. By necessity, this sketch is incomplete as it is more than what can be accomplished in a Ph.D., but we hope that it is compelling in its scope.

\subsection{Prior Publication}

Parts of this thesis have been published. Chapters 2 and 4 were published in the Proceedings of the 34th Annual Conference of the Cognitive Science Society (Kelly \& West, 2012). Table 2.1 of Chapter 2 is a modified version of a table previously published in an article in Canadian Journal of Experimental Psychology (Kelly, Blostein, \& West, 2013). An earlier version of Chapter 5 was published in the Proceedings of the 36th Annual Conference of the Cognitive Science Society (Kelly, Mewhort, \& West, 2014). Chapter 5 has since been submitted, reviewed, revised, and resubmitted for publication with a journal. Chapter 7 was published in the Proceedings of the 13th International Conference on Cognitive Modeling (Kelly, Kwok, \& West, 2015). Chapter 8 was published in the Proceedings of the 12th International Conference on Cognitive Modeling (Kelly \& West, 2013), and as part of a longer journal article in Biologically Inspired Cognitive Architectures (Rutledge-Taylor, Kelly, West, \& Pyke, 2014). 


\section{Chapter 2 Vectors To Symbols: The Sub-Symbolic Level}

To achieve a full, theoretical understanding of a cognitive process, explanations of the process need to be provided at both symbolic (i.e., representational) and sub-symbolic levels of description. We argue that cognitive models implemented in vector-symbolic architectures (VSAs) intrinsically operate at both of levels and thus provide a needed bridge. We characterize the sub-symbolic level of VSAs in terms of a small set of linear algebra operations.

\subsection{Introduction}

Developed in the 1960s and 1970s, the symbol processing models are the earliest mechanistic models of cognition. The LISP (list processing) language, developed in 1958, became popular in the budding fields of artificial intelligence and cognitive modelling. LISP programs operate on lists of discrete symbols. LISP heavily influenced the nature of early cognitive models, such as the cognitive architecture ACT-R, which is standardly implemented in LISP.

Symbolic models are systems most appropriately described at the level of expressions of symbols and the manipulation of those symbols (Clark, 2001). Symbolic models take inspiration from linguistics and logic. Typically, in a symbolic architecture, the atomic units of the model are concepts (e.g., dog, glowing, noun, or anger) and complex units arise from characterizing the relations between those concepts. Arguably, John Locke (1690) proposed one of the first symbolic architectures, well before there were computers, analyzing the mind in terms of a set of processes that manipulate expressions of elementary ideas. 
With the advent of parallel distributed processing in the 1980s, neurally-inspired connectionist models became a popular alternative to traditional symbolic models. By contrast to symbolic models, the atomic units of a connectionist system are artificial neurons or simple neuron-like nodes, whereas a concept will have a complex implementation as a pattern of activation across those units. Connectionist models transform vectors of activation values using matrices of connection weights. While the patterns of activation at the input or output of a connectionist model may be interpretable as a known concept, the patterns of activation on the hidden layers of neurons or nodes between the input and output are often difficult to interpret.

To achieve a full, theoretical understanding of a cognitive process and how it relates to the physical world, explanations of the process need to be provided at both symbolic (i.e., representational) and sub-symbolic levels of description. The classic symbolic approaches to modelling do not account for how the symbol manipulations described in the model could arise from neural tissue, or account for how the symbols themselves come into existence. Classic connectionist approaches are more concerned with neural plausibility, but are notoriously opaque, doing little to aid our understanding of the cognitive processes modelled. By contrast, the vector-symbolic approach to modelling explicitly provides an account at both symbolic and sub-symbolic levels of description.

In vector symbolic architectures, the vectors represent symbolic information. These vectors, or symbols, can be combined and manipulated using a small number of operations, which can be understood as sub-symbolic processes. However, the information processing models built from these operations are best characterized at a symbolic level of description. Importantly, the modelling decisions made at the sub-symbolic level are to some degree 
independent of the modelling decisions made at the symbolic level. This chapter discusses the sub-symbolic level. Chapter 4 discusses the symbolic level.

Table 2.1. Comparison of VSAs and tensor representations.

\begin{tabular}{|c|c|c|c|c|c|}
\hline $\begin{array}{l}\text { Vector Symbolic } \\
\text { Architecture }\end{array}$ & Representation & Binding & $\begin{array}{l}\text { Binding Time } \\
\text { Complexity }\end{array}$ & Superposition & $\begin{array}{l}\text { Unbinding } \\
\text { Accuracy }\end{array}$ \\
\hline $\begin{array}{l}\text { Tensor representations } \\
\text { (Smolensky, 1990) }\end{array}$ & $\begin{array}{l}\text { rank } k \text { tensor of } n^{k} \text { real } \\
\text { values }\end{array}$ & tensor product & $\mathrm{O}\left(n^{k}\right)$ & addition & 1 \\
\hline $\begin{array}{l}\text { HRRs } \\
\text { (Plate, 2003, chap. 3) }\end{array}$ & vector of $n$ real values & $\begin{array}{l}* \text {, circular } \\
\text { convolution }\end{array}$ & $\mathrm{O}(k n \log n)$ & addition & 0.71 \\
\hline $\begin{array}{l}\text { Frequency-domain HRRs } \\
\text { (Plate, 2003, chap. 4) }\end{array}$ & $\begin{array}{l}\text { vector of } n \text { complex } \\
\text { values on the unit circle }\end{array}$ & $\begin{array}{l}\text { ॰, element-wise } \\
\text { multiplication }\end{array}$ & $\mathrm{O}(k n)$ & $\begin{array}{l}\text { normalized } \\
\text { addition }\end{array}$ & 1 \\
\hline $\begin{array}{l}\text { Binary spatter codes } \\
\text { (Kanerva, 1996) }\end{array}$ & $\begin{array}{l}\text { vector of } n \text { binary } \\
\text { values }\end{array}$ & bit-wise XOR & $\mathrm{O}(k n)$ & majority & 1 \\
\hline $\begin{array}{l}\text { MAP coding } \\
\text { (Gayler, 2003) }\end{array}$ & $\begin{array}{l}\text { vector of } n \text { real values, } \\
\text { initially }+1 \mathrm{~s} \text { and }-1 \mathrm{~s}\end{array}$ & $\begin{array}{l}\circ \text {, element-wise } \\
\text { multiplication }\end{array}$ & $\mathrm{O}(k n)$ & $\begin{array}{l}\text { normalized } \\
\text { addition }\end{array}$ & 1 \\
\hline $\begin{array}{l}\text { Square matrix } \\
\text { representations } \\
\text { (Kelly, 2010) }\end{array}$ & $\begin{array}{l}n^{1 / 2} \times n^{1 / 2} \text { square matrix } \\
\text { of } n \text { real values }\end{array}$ & $\begin{array}{l}\text { matrix } \\
\text { multiplication }\end{array}$ & $\mathrm{O}\left(k n^{3 / 2}\right)$ & addition & 0.70 \\
\hline $\begin{array}{l}\text { Used in CHARM } \\
\text { (Eich, 1982) }\end{array}$ & vector of $n$ real values & $\begin{array}{l}\text { truncated } \\
\text { aperiodic } \\
\text { convolution }\end{array}$ & $\mathrm{O}(k n \log n)$ & addition & 0.70 \\
\hline $\begin{array}{l}\text { Geometric analogues of } \\
\text { HRRs (Aerts, Czachor, \& } \\
\text { De Moore, 2009) }\end{array}$ & $\begin{array}{l}\text { multivector of } \\
\sum_{i=0}^{\left\lfloor\frac{k}{2}\right\rfloor}\left(\begin{array}{l}n \\
2 i+(k \bmod 2)\end{array}\right) \\
\text { real values }\end{array}$ & $\begin{array}{l}\text { geometric } \\
\text { product }\end{array}$ & not reported & addition & $\begin{array}{l}\text { not } \\
\text { reported }\end{array}$ \\
\hline
\end{tabular}

Note. Complexity expressed in terms of vectors (or square matrices) of $n$ values and the number of vectors associated together, $k$. An earlier version of this table appeared as Table 1 in Kelly, Blostein, and Mewhort (2013).

\subsection{The Sub-Symbolic Level}

VSAs are closely related to tensor product representations (Smolensky, 1990), but unlike tensor product representations, VSAs can compactly represent symbolic expressions of arbitrary complexity. A number of VSA techniques exist in the literature, including Holographic Reduced 
Representations (HRRs; Plate, 1995), frequency-domain HRRs (Plate, 2003, chap. 4), the geometric analogue to HRRs (Aerts, Czachor, \& De Moore, 2009), some earlier forms of holographic associative memory (Eich, 1982; Murdock, 1982), as well as binary spatter codes (Kanerva, 1992), Multiply-Add-Permute coding (Gayler, 2003), and square matrix representations (Kelly, 2010). See Table 2.1 for an overview of the different VSAs.

Each VSA technique uses the same set of basic operations, but implements the operations differently. Thus the choice of a particular VSA dictates how symbols are instantiated and manipulated and defines the model at the sub-symbolic level. To ground the discussion, we mainly discuss Holographic Reduced Representations (HRRs; Plate, 1995), as HRRs are the most widely used VSAs in the cognitive modelling literature. Also, HRRs are used as the basis for the Neural Engineering Framework (NEF; Eliasmith, 2013) and thus demonstrably have a clearly defined and plausible neural implementation. However, the other VSA techniques are similar and most anything that can be done with an HRR can be done with any VSA technique.

\section{3 $n$-Space and Similarity}

In a VSA, a symbol, or representation, is an $n$-dimensional vector: a list of $n$ numbers that defines the coordinates of a point in an $n$-dimensional space. VSAs work best for values of $n$ in the hundreds or thousands (Plate, 1995).

A vector can be understood as a line drawn from the origin (the zero coordinates) to the coordinates specified by the vector. The length of the line is the vector's magnitude. The direction of the vector encodes the meaning of the representation. Similarity in meaning can thus be measured by the size of the angles between vectors. This is typically quantified as the cosine of 
the angle between vectors (Goebel \& Lewandowsky, 1991). The cosine of vectors a and b can be calculated as:

$$
\operatorname{cosine}(\mathbf{a}, \mathbf{b})=(\mathbf{a} \cdot \mathbf{b}) /\left((\mathbf{a} \cdot \mathbf{a})^{0.5}(\mathbf{b} \cdot \mathbf{b})^{0.5}\right)
$$

where $\bullet$ is the dot product. A cosine of 1 means the vectors are identical, -1 means they are opposites, and 0 means they are completely dissimilar. If each vector has a magnitude of one, the cosine is just the dot-product of the vectors. Thus, some systems rescale all vectors to a magnitude of one after vector operations. In memory systems where new memories are superimposed on old memories, such re-scaling causes a recency effect and rapid forgetting because new memories will make-up a fixed fraction of memory, regardless of the quantity of previous experience.

While the cosine measures the angle between two vectors, the cosine is often described as a measure of distance. As it is more intuitive to describe similarity as a measure of distance than as a measure of the angle, for convenience, we can imagine the vectors as describing points on a hypersphere, such that the size of the angles are the distances between them.

\subsection{Atomic versus Complex representations}

Representations in a VSA are either atomic or complex. An atomic representation is a unique representation, a symbol that cannot be broken down into sub-symbols. In an HRR, values for an atomic representation are typically generated by random sampling from a standard normal distribution. By assigning random values to the vectors, atomic representations will be uniformly 
distributed across the surface of the hypersphere, such that the atomic representations will have little to no similarity to each other.

Complex representations can be created by either combining atomic representations or recursively combining complex representations. Critically, in a VSA, a complex representation has the same dimensionality as an atomic representation, allowing representations both atomic and complex to be compared, or combined together to create representations of arbitrary complexity. VSAs have two operators for combining representations: superposition and binding. In HRRs, superposition is vector addition and binding is circular convolution. We denote vector addition by + , and circular convolution by *. Binding and superposition, along with random permutation, are the basic operations used to create complex representations in VSAs.

\subsection{Superposition (+) versus Binding (*)}

The key difference between superposition and binding is their effect on similarity. Superposition is similarity-preserving: the sum of two vectors is a vector that falls in the angle between them. Conversely, binding is similarity destroying: the circular convolution of two vectors is roughly orthogonal to the two original vectors. The purpose of superposition is to combine representations to create a new representation that is similar to all of the combined representations. The purpose of binding, on the other hand, is to create "chunks": unique identifiers for combinations of representations.

Most VSA use a form of vector addition for superposition. Vector addition is computed by adding together the corresponding elements of the two vectors. So, for example, $(1,4,7)+$ $(5,4,2)=(6,8,9)$. 
To bind, HRRs use circular convolution, * , which can be computed rapidly using element-wise multiplication, ${ }^{\circ}$, and the fast Fourier transform, $f f t$, and its inverse, $f f t^{-1}$ :

$$
\mathbf{a}^{*} \mathbf{b}=\mathrm{fft}^{-1}(\mathrm{fft}(\mathbf{a}) \circ \mathrm{fft}(\mathbf{b}))
$$

Essentially circular convolution is a lossy way of scrambling the information of the two vectors together to produce a new vector of the same dimensionally. The mathematical operation used varies from one VSA to another (see Table 2.1), but all binding operations are a "multiplicationlike” operator (Gayler, 2003).

Consider the problem of learning the meaning of the phrase "kick the bucket", a colloquial euphemism for death. Suppose the cognitive model has a vector representation of the concept kick and a vector representation of the concept bucket. The sum (superposition) of those two vectors will produce a vector that is close to both kick and bucket, indicating that the phrase "kick the bucket" has a meaning similar to kick and to bucket. But in order for the cognitive model to be able to learn that the phrase "kick the bucket" has a distinct meaning that is not a function of its parts, the model needs to be able to assign to "kick the bucket" a distinct identifier. Binding is the operation that performs this function in VSA-based models. The vector kick* bucket is dissimilar to the vectors kick, bucket, and kick + bucket.

Binding and superposition can also be used jointly to address the binding problem (Gayler, 2003), that is, the question of how to couple sets of attributes together such that the attributes of one object are not confused with the attributes of another. For example, given a small red square and a large blue circle, the complex representation (small * red * square) + 
(large * blue * circle) creates a single vector that distinctively represents the knowledge that the square is small and red and the circle is large and blue.

\subsection{Unbinding}

Unbinding is an inverse of binding that allows vectors that have been bound together in a complex representation to be unpacked and recovered. Circular correlation, \#, is the unbinding operator for HRRs. Given a pair of vectors bound together, and one of the pair, referred to as the probe, unbinding produces an approximation of the other vector, referred to as the target, i.e.,

$$
\mathbf{p} \#(\mathbf{p} * \mathbf{t})=\mathbf{t}^{\prime} \approx \mathbf{t}
$$

where $\mathbf{p}$ is the probe, $\mathbf{t}$ is the target, and $\mathbf{t}$ ' is an approximation of the target. The similarity between $\mathbf{t}$ and $\mathbf{t}$ ' varies depending on the choice of VSA. Table 2.1 shows the cosine similarities between $\mathbf{t}$ ' and $\mathbf{t}$ for various VSAs.

Unbinding can be understood as binding with the inverse of the probe. The inverse of any vector $\mathbf{x}$ is a re-ordering of the elements of $\mathbf{x}$, i.e. a permutation of $\mathbf{x}$, such that,

$$
\mathbf{x} \# \mathbf{x}=\mathbf{x} * \operatorname{inverse}(\mathbf{x}) \approx \boldsymbol{\delta}
$$

where $\boldsymbol{\delta}$ is the identity vector for binding, i.e. for any vector $\mathbf{x}, \mathbf{x} * \boldsymbol{\delta}=\mathbf{x}$. Thus binding with the inverse of a vector unbinds what that vector has been associated with: 


$$
\mathbf{a} \#(\mathbf{a} * \mathbf{b})=\operatorname{inverse}(\mathbf{a}) *(\mathbf{a} * \mathbf{b}) \approx \boldsymbol{\delta} * \mathbf{b}=\mathbf{b}
$$

In HRRs, the inverse of any vector $\mathbf{x}=\left(\mathbf{x}_{1} \ldots \mathbf{x}_{\mathrm{n}}\right)$ is:

$$
\operatorname{inverse}(\mathbf{x})=\left(\mathbf{x}_{1}, \mathbf{x}_{\mathrm{n}}, \mathbf{x}_{\mathrm{n}-1}, \ldots \mathbf{x}_{3}, \mathbf{x}_{2}\right)
$$

Circular convolution, *, is commutative, i.e., the order of binding does not matter when using circular convolution. Given vectors $\mathbf{a}$ and $\mathbf{b}$, their association $\mathbf{a} * \mathbf{b}=\mathbf{b} * \mathbf{a}$, and likewise, when unbinding, $\mathbf{b} \#(\mathbf{a} * \mathbf{b})=\mathbf{b} \#(\mathbf{b} * \mathbf{a}) \approx \mathbf{a}$.

\subsection{Permutation}

A permutation matrix $\mathbf{P}$ is an $n \times n$ matrix obtained by permuting the rows of the identity matrix (i.e., a matrix where the entries along the main diagonal are ones and all other entries are zeros) according to a permutation of the numbers $1 \ldots n$. Permuting a vector a can be expressed as multiplying by a permutation matrix $\mathbf{P}$, i.e., $\mathbf{P}$ a. A randomly chosen permutation of a vector is unlikely to be similar to the original vector, $\operatorname{cosine}(\mathbf{P} \mathbf{a}, \mathbf{a}) \approx 0$. The permutation is also reversible. Given the permutation $\mathbf{P}$, the transpose $\mathbf{P}^{\mathrm{T}}$ is such that $\mathbf{P}^{\mathrm{T}}(\mathbf{P}(\mathbf{a}))=\mathbf{a}$.

Gayler (2003) describes random permutation as an operation used "to quote or protect the vectors from the other operations". When permuted, the information within a vector is essentially hidden and protected from being affected by other vector operations. For example, as noted above, circular convolution is commutative, that is, $\mathbf{a}^{*} \mathbf{b}=\mathbf{b}^{*} \mathbf{a}$. This property of circular convolution can be useful, but it can be a hindrance in situations where the order of items matter, 
e.g. "dog feed" and "feed dog" are phrases which carry different meanings by virtue of differences in word order.

A non-commutative variant of circular convolution can be defined using a random permutation $\mathbf{P}$ and its inverse $\mathbf{P}^{\mathrm{T}}$. By always randomly permuting one of the arguments before convolution, one defines a binding operation that is non-commutative, i.e. while $\mathbf{a}^{*} \mathbf{b}=\mathbf{b}^{*} \mathbf{a},(\mathbf{P}$ $\mathbf{a})^{*} \mathbf{b} \neq(\mathbf{P} \mathbf{b})^{*} \mathbf{a}$. Unbinding then uses the inverse permutation $\mathbf{P}^{\mathrm{T}}$, e.g.

$$
\begin{aligned}
& \operatorname{cosine}\left(\mathbf{P}^{\mathrm{T}}(\mathbf{a} \#(\mathbf{a} *(\mathbf{P} \mathbf{b}))), \mathbf{b}\right) \approx 0.71 \\
& \operatorname{cosine}\left(\mathbf{P}^{\mathrm{T}}(\mathbf{b} \#(\mathbf{a} *(\mathbf{P} \mathbf{b}))), \mathbf{a}\right) \approx 0
\end{aligned}
$$

Non-commutative binding is used by the BEAGLE model (Jones \& Mewhort, 2007) to bind vectors that stand for words in sentences in order to construct representations of the semantics of each of those words. For a variety of other uses of random permutation in VSAs, see Gayler (2003), Sahlgren, Holst, and Kanerva (2008), and Kelly, Blostein, and Mewhort (2013). 


\section{Chapter 3 Addressing Criticisms of Connectionist and Symbolic Models}

AI and cognitive modelling have historically been divided into connectionist and symbolic approaches, each with their respective merits and demerits. Vector symbolic architectures (VSAs), such as holographic reduced representations (HRRs), can be understood as a scheme for knowledge representation with qualities of both approaches.

In this chapter, we outline the advantages of the VSA approach. Unlike traditional connectionist approaches, VSAs can represent knowledge with complicated or recursive structure. Unlike traditional symbolic approaches, VSAs are easily implemented in neural models, and easily scaled to large problems. Whether VSAs can be applied to problem domains that cannot be trivially discretized into symbols remains an open question.

\subsection{Addressing Criticism of Connectionism}

VSAs and tensor representations are motivated in the literature as an answer to criticisms of connectionism (Gayler, 2003; Plate, 1995; Smolensky, 1990). Artificial neural networks (i.e., connectionist models) have often been criticized for an inability to represent or manipulate knowledge with complicated or recursive structure (Fodor \& Pylyshyn, 1988; Hinton, 1988; Jackendoff 2002). VSAs provide an approach to knowledge representation that can easily be implemented in realistic neural models (Stewart \& Eliasmith, 2008). VSAs address Fodor and Pylyshyn's (1988) productivity critique, are capable of representing XOR, implement Hinton's solution (1988) to the part-whole problem (Plate, 1995), and may provide a basis for a unified theory of working and long-term memory (Gayler, 2003). 


\subsection{Productivity Critique}

Productivity is a term from grammar theory, meaning the ability of a grammar to produce new utterances. A grammar is infinitely productive if it can produce an infinite number of utterances over a finite alphabet. Viewed as a grammar, a simple associative memory, such as Hopfield network or one-layer neural network, is not infinitely productive: given a finite set of atomic structures $\mathbf{a} \ldots \mathbf{z}$, it can only produce the associations $\mathbf{a a}, \mathbf{a b}, \mathbf{a c}, \ldots \mathbf{z x}, \mathbf{z y}, \mathbf{z z}$, that is, all possible pairs, which is a finite number of associations.

Alternatively, given a vector $\mathbf{a}$, one can use the tensor product $\otimes$ to produce an infinite number of associations: $\mathbf{a}, \mathbf{a} \otimes \mathbf{a}, \mathbf{a} \otimes \mathbf{a} \otimes \mathbf{a}, \mathbf{a} \otimes \mathbf{a} \otimes \mathbf{a} \otimes \mathbf{a}, \ldots$ etc. Thus, tensor representations define an infinitely productive grammar and address Fodor and Pylyshyn's (1988) productivity critique.

Productivity gives tensor representations and VSAs a great deal of power. Famously, Minsky and Papert (1969) demonstrated that single-layer perceptrons are incapable of modelling exclusive disjunction (XOR). In spite of their simplicity, tensor representations and VSAs such as HRRs can model XOR (Kelly, 2010). Given three atomic structures, $\mathbf{a}, \mathbf{b}$, and $\mathbf{x}$, respectively representing "A is true", "B is true", and "Either A or B is true, but not both", and a random permutation representing not, the complex structure m models XOR, where,

$$
\mathbf{m}=\left(\mathbf{a}^{*} \mathbf{b}^{*}\left(\mathbf{P}_{\text {not }} \mathbf{x}\right)\right)+\left(\mathbf{a}^{*}\left(\mathbf{P}_{\text {not }} \mathbf{b}\right) * \mathbf{x}\right)+\left(\left(\mathbf{P}_{\text {not }} \mathbf{a}\right) * \mathbf{b}^{*} \mathbf{x}\right)+\left(\left(\mathbf{P}_{\text {not }} \mathbf{a}\right)^{*}\left(\mathbf{P}_{\text {not }} \mathbf{b}\right)^{*}\left(\mathbf{P}_{\text {not }} \mathbf{x}\right)\right)
$$

Given any of the four possible pairs of inputs, $\mathbf{m}$ can be used to retrieve the correct output, 


$$
\begin{aligned}
& (\mathbf{a} * \mathbf{b}) \# \mathbf{m} \approx \mathbf{P}_{\mathrm{not}} \mathbf{x} \\
& \left(\mathbf{a} *\left(\mathbf{P}_{\mathrm{not}} \mathbf{b}\right)\right) \# \mathbf{m} \approx \mathbf{x} \\
& \left(\left(\mathbf{P}_{\mathrm{not}} \mathbf{a}\right) * \mathbf{b}\right) \# \mathbf{m} \approx \mathbf{x} \\
& \left(\left(\mathbf{P}_{\mathrm{not}} \mathbf{a}\right) *\left(\mathbf{P}_{\mathrm{not}} \mathbf{b}\right)\right) \# \mathbf{m} \approx \mathbf{P}_{\mathrm{not}} \mathbf{x}
\end{aligned}
$$

\subsection{Part-Whole Problem}

While very similar, a distinction can be made between VSAs and tensor representations in terms

of representational power. Tensor representations do not address the part-whole problem (Hinton, 1988). Imagine a room in which there is a picture on the wall, and that picture is of the room.

How can one design a system that can recognize that the room one is in and the room depicted in the picture are the same? Suppose we represent the room, r, as the association of all the things in the room. Suppose the picture on the wall is $\mathbf{p}$ and the other stuff in the room is $\mathbf{s}$. Then, $\mathbf{r}=\mathbf{s} \otimes$ $\mathbf{p}$, where $\otimes$ is the tensor product. The tensor $\mathbf{s}$ is of rank equal to the number of things in the room other than the picture. The tensor of $\mathbf{r}$ is of rank equal to the rank of $\mathbf{s}$ plus the rank of $\mathbf{p}$. Since the picture on the wall is of the room, $\mathbf{p}=\mathbf{r}$. Thus, $\mathbf{r}=\mathbf{s} \otimes \mathbf{r}$, and $\mathbf{r}$ must be a tensor of infinite rank, which is not an easily computable representation.

Hinton (1988) proposes a solution: use reduced representations to represent parts. The reduced descriptions can then be expanded into full descriptions should those parts become the focus of attention. The description of the room contains a reduced description of the picture on the wall. When the description of the picture is expanded, that description is identical (or nearly so) to the description of the room, allowing the system to recognize an identity 
between the room and the picture. Because the dimensions of a VSA does not grow with the number of associations, VSAs implement Hinton's reduced representations.

\subsection{Binding in Working v.s. Long-Term Memory}

Jackendoff (2002) argues that language challenges the traditional long-term versus working memory distinction. Jackendoff notes that linguistic structures need to be rapidly accessed and manipulated, but also remembered for a long time. It is commonly believed that working memory is instantiated by fast-changing patterns of neural activation, whereas long-term memory is instantiated by slow-changing connections between neurons. Jackendoff speculates that patterns of activation are incapable of representing the complexity of language, and synaptic connections change too slowly to allow for the rapidity with which we process language. Jackendoff notes this poses a problem not just for modelling language, but for any domain requiring rapid processing of complex representations, such as vision.

Gayler (2003) observes that VSAs could provide a solution to this problem. Encoding an association in VSAs is a vector operation, and does not need iteration, thus VSAs are a fast, plausible algorithm for how working memory (i.e., patterns of activation in the brain) could represent knowledge with complicated structure. Furthermore, VSA representations could also be encoded in synaptic connections, such that VSAs could provide a basis for uniting long-term and working memory.

In spite of the difference in neural implementation, if both long-term and working memory represented information using VSAs, there would be little functional difference between the two systems. The definitional functional difference between representations in working and 
long-term memory is the longevity of the representations. Using VSAs to model both long-term and working memory, the difference between these systems can be reduced to the definitional, which naturally arises as a consequence of the difference in neural implementation. Indeed, the ability of VSA-based models to simulate human behaviour in both long-term (e.g. memory for word meaning; Jones \& Mewhort, 2007) and short-term memory tasks (e.g. rock-paper-scissors; Rutledge-Taylor et al., 2014) suggests that the functional difference between these memory systems may be minimal.

\subsection{Vector Symbolic versus Symbolic}

Why bother with vector algebra if a VSA can be fully understood in terms of symbol manipulation? Consider manipulating lists of symbols, as in LISP and LISP implemented cognitive models and architectures such as ACT-R (Anderson \& Lebiere, 1998).

Both list-based models, such as ACT-R, and VSA-based models, such as TODAM (Murdock, 1982) and DHSM (Rutledge-Taylor et al., 2014), have an established ability to model how human behaviour is controlled by knowledge. However, VSAs, by implementing symbols as vectors, provide a bridge between low-level neural models and high-level symbolic models (e.g. Stewart \& Eliasmith, 2008).

Furthermore, as lossy representations, VSAs are practical when the amount of data to be stored is large. If the VSA stores its data in $m$ complex structures of dimensionality $n$, the time and space complexity for storage and retrieval of data will be a function of $m$ and $n$, irrespective of the amount of data put into the system. VSAs are thus scalable to large problems, such as representing the word-meaning knowledge that can be gleaned from a corpus of text equivalent 
in size to the amount of material a college-level student has read in their lifetime (Jones \& Mewhort, 2007).

Conversely, in a loss-less representation, the time and space demands of the system grow with the amount of data stored. Referred to as the utility problem (Minton, 1990), many cognitive architectures become impractical as the quantity of data in their long-term memory grows (Langley, Laird, \& Rogers, 2009). Rutledge-Taylor and West (2007) suggest using an HRRbased system as a solution to the utility problem for ACT-R.

The term 'lossy' in the context 'VSAs are lossy representations' is perhaps misleading. While data is lost, that loss can itself be a gain. As a lossy compression of a set of associations, a complex structure in a VSA is a generalization. By storing all the associations between a word and other words from a large sample of the language, a complex structure can be used to represent the meaning of that word (Jones \& Mewhort, 2007). The inaccuracy of retrieval from an VSA can be used to model the behaviour of human memory (Eich, 1982; Murdock, 1983). VSAs are interesting by virtue of their power not just to act as a knowledge representation, but as a mechanism for discovering the underlying function in the data.

\subsection{Best of Both Worlds?}

If VSAs are to address criticism of connectionism, VSAs should also retain the strengths of connectionism, or else we are simply substituting the weaknesses of one system for the weaknesses of another. Can VSAs accomplish all the things that connectionism is lauded for? This question has largely not been addressed by the VSA or HRR literature. 
Can VSAs be used to implement a perceptual system? Or, given a perceptual system, can a VSA operate on the vectors produced by that system? VSA-based models generally operate on vectors of random values. An atomic structure is generated randomly - it is truly and purely a symbol, as it contains no information about what it represents. A vector from the environment produced by a sensory device, or a vector passed to the VSA by a perceptual system, will contain information about what it represents.

Using vectors of structured, representative data as atomic structures in HRRs is explored by Kelly, Blostein, and Mewhort (2013) and it raises interesting questions, such as the importance of a model of attention, and the power of VSAs to do function discovery. Given a set of vectors $\mathbf{x}_{1} \ldots \mathbf{x}_{\mathrm{n}}$ and a function $f$, using circular convolution $*$, the structure $\mathbf{x}_{1} * f\left(\mathbf{x}_{1}\right)+\ldots+\mathbf{x}_{\mathrm{n}}$ $* f\left(\mathbf{x}_{\mathrm{n}}\right)$, approximates the function $f$, if $f$ is a linear function (Kelly, 2010). If $f$ is a non-linear function, is there a good algorithm for constructing a complex structure in a VSA to represent that function? As the process of perception can be understood as developing internal models of the functions in the environment that produce sensations, the ability of a VSA-based model to do function discovery may be critical to its power as a model of the human mind.

\subsection{Conclusions}

VSAs provide a promising bridge between connectionist and symbolic approaches to building cognitive models. VSAs retain the expressive power of symbolic approaches, with the added benefit of being lossy representations. As lossy, fixed dimensional representations, VSAs are easily scalable to large problems (e.g. Jones \& Mewhort, 2007), and the error introduced by their lossy nature makes them suited to modelling human memory (e.g., Eich, 1982; Murdock, 1982; 
Rutledge-Taylor et al., 2014; Franklin \& Mewhort, 2015). As symbols in VSAs are represented as vectors that can be understood as patterns of activation, VSAs can be implemented in realistic neural models (e.g. Stewart \& Eliasmith, 2008). VSAs are agnostic as to whether the vectors are implemented in patterns of neural activation or encoded in synaptic connectivity. As a result, both working memory and long-term memory could be described by an integrated VSA architecture.

While VSAs are generally considered a connectionist approach, and motivated by the need for a mechanism to express complicated structure in connectionist models, little has been done to demonstrate that VSAs retain the strengths of connectionist models. While VSAs can represent non-linear functions, can VSAs be used for non-linear function discovery? Can a VSAbased model be designed to interface with a perceptual, rather than symbolic, environment? 


\section{Chapter 4 Symbols to Cognition: The Symbolic Level}

We characterize the symbolic level of VSAs in terms of cognitive processes, in particular how information is represented, stored, and retrieved. We classify vector-symbolic cognitive models in the literature according to their implementation of these processes. On the basis of our analysis, we suggest means for theoretical unification of existent models.

\subsection{The Symbolic Level}

When making a vector-symbolic model, decisions need to be made at both the symbolic and subsymbolic levels. At the sub-symbolic level, the modeller needs to decide how to instantiate symbols as vectors and symbol-manipulation as vector algebra. Conversely, at the symbolic level, the modeller needs to make decisions about how to structure, manage, store, and retrieve those symbols. Choosing to use HRRs rather than another kind of VSA can define the subsymbolic level, but this choice is largely independent of the decisions to be made at the symbolic level.

In Chapter 2, there were two examples of manipulations at the symbolic level. The first was combining binding and addition to create a vector that encodes information about bound entities (e.g., small red square and large blue circle). The second was combining permutation and binding to create a bound entity that maintained information about order. Essentially, all VSA systems work in the same way. Vectors encode the desired information according to some sort of scheme (i.e., by combining the operations discussed above), and then, when needed, the information is retrieved from the vectors. 


\subsection{Encoding and Storage}

BEAGLE (Jones \& Mewhort, 2007) and DSHM (Rutledge-Taylor et al., 2014) use the terms environment vectors and memory vectors. We extend the use of this terminology to other vectorsymbolic models. An environment vector is a vector that stands for atomic perceptions from the environment (e.g., a red circle needs two environment vectors, one for circle and one for red). Environment vectors are fixed and do not change. A memory vector is a complex representation stored by the model and used to produce behaviour. In some systems, memory vectors change with experience. Additionally, we use the term experience vector to refer to a representation that stands for the model's current experience of its environment created by combining environment vectors (e.g., an experience vector could represent the perception of a red circle by convolving the environment vectors of circle and red).

By examining the relationship between environment, experience, and memory vectors across vector-symbolic models, we distinguish between three main approaches to storage. In a many-to-one vector model, all experience vectors are summed into a single memory vector for storage. In a one-to-one vector model, each experience vector is stored as a separate memory vector among an ever-growing number of memory vectors. In a many-to-many vector model, there are a fixed number of memory vectors, and incoming environment vectors are used to update them. Each of these approaches has strengths and weaknesses.

Many-to-one In a many-to-one vector model, such as TODAM (Murdock, 1983) or CHARM (Eich, 1982), memory is modelled as a single, high-dimensional vector. All experience vectors 
are added to the memory vector. There is a limit to how much can be stored in the vector before mistakes start to be made. Mistakes are, of course, of interest to psychologists, and the pattern of mistakes made by a many-to-one vector model allow it to mimic human forgetting in list-recall tasks. If the goal is to model how people store a small amount of recently learned or closely related information, a single memory vector suffices.

Many-to-one vector models also have the advantage of a clear neural implementation. In the Neural Engineering Framework (NEF; Eliasmith, 2013) binding, unbinding, and superposition can all be implemented through neural connectivity. In the NEF interpretation, a many-to-one memory is a neural group with self-recurrent connections that acts as a working memory or buffer, and many such buffers could exist in the brain.

One-to-one In a one-to-one vector model, such as MINERVA (Hintzman, 1986), the Iterative Resonance Model (Mewhort \& Johns, 2005), and the Holographic Exemplar Model (Jamieson \& Mewhort, 2011), each experience vector is represented as a separate memory. While this approach to modelling memory is both simple and successful, the ever growing number of vectors that need to be stored and accessed by the memory system seems neurally implausible and computationally impractical for modelling tasks in which very large amounts of knowledge are relevant, e.g., semantic priming tasks (Jones \& Mewhort, 2007). However, these models are able to reproduce a wide variety of memory effects, providing a unitary account of episodic, semantic, and implicit memory, indicating that, although their warehouse-style management of vectors seems implausible, their processes of storage and retrieval provide a good analogue for biological memory. 
Many-to-many Many-to-many vector models, such as BEAGLE (Jones \& Mewhort, 2007) and DSHM (Rutledge-Taylor et al., 2014), can be understood as a hybrid of the earlier many-to-one and one-to-one approaches. In many-to-many memory, for each item of interest, there is a randomly generated environment vector and a specially constructed memory vector. In BEAGLE the items of interest are words: the environment vector stands for the word's orthography or phonology and the memory vector stands for the word's meaning. In DSHM, the items are objects relevant to the experimental task: the environment vector stands for the percept of the object and the memory vector stands for the concept of the object.

Like the one-to-one models, the management of the vectors in many-to-many systems is computationally expensive and also seems neurally implausible. However, the ability to generate memory vectors that stand for particular concepts in very powerful (e.g., Rutledge-Taylor, Vellino, \& West, 2008) and allows these systems to capture numerous different phenomena (e.g., Rutledge-Taylor et al., 2014) and represent vast quantities of data (Jones \& Mewhort, 2007).

For example, to create an association between keyboards and computers, each time a computer and keyboard co-occur a copy of the environment vector for keyboard can be added to the memory vector for computer and a copy of the environment vector for computer can be added to the memory vector for keyboard. The effect of this would be to move the memory vector for computer closer to the environment vector for keyboard and move the memory vector for keyboard closer to the environment vector for computer. Over time, the result of this is to organize the space so that memory vectors are clustered around environment vectors that they co-occur with so that the distance between the vectors equals strength of association. 
Another, more complicated example involves binding and the use of the placeholder vector, denoted by $\boldsymbol{\Phi}$. The placeholder vector is an atomic (i.e, random) vector, but it is used to encode all associations, and thus can be used as a universal retrieval cue. Consider the phrase or stimulus blue triangle. Without using the placeholder, we could update memory as follows:

$$
\begin{aligned}
& \mathbf{m}_{\text {blue }, t+l}=\mathbf{m}_{\text {blue }, t}+\mathbf{e}_{\text {blue }} * \mathbf{e}_{\text {triangle }} \\
& \mathbf{m}_{\text {triangle, } t+l}=\mathbf{m}_{\text {triangle }, t}+\mathbf{e}_{\text {blue }} * \mathbf{e}_{\text {triangle }}
\end{aligned}
$$

where $\mathbf{m}$ is a memory vector, $\mathbf{e}$ is an environment vector and $t$ is the timestep in the model. By binding together the environment vectors for blue and triangle and adding the result to the memory vectors for blue and triangle, we move the two memory vectors towards the point in space described by the vector blue * triangle, and thereby move $\mathbf{m}_{\text {blue }}$ and $\mathbf{m}_{\text {triangle }}$ closer together. But people almost never get the concepts blue and triangle confused with each other. This is because blue is a colour (or an adjective), and triangle is a shape (or a noun), i.e. they are different sorts of thing.

Conversely, consider updating using the placeholder:

$$
\begin{aligned}
& \mathbf{m}_{\text {blue, } t+1}=\mathbf{m}_{\text {blue }, t}+\boldsymbol{\Phi} * \mathbf{e}_{\text {triangle }} \\
& \mathbf{m}_{\text {triangle, } t+l}=\mathbf{m}_{\text {triangle }, t}+\mathbf{e}_{\text {blue }} * \boldsymbol{\Phi}
\end{aligned}
$$

This moves $\mathbf{m}_{\text {blue }}$ towards $\boldsymbol{\Phi} * \mathbf{e}_{\text {triangle, }}$ i.e. towards all properties of triangles, and moves $\mathbf{m}_{\text {triangle }}$ towards $\mathbf{e}_{\text {blue }} * \boldsymbol{\Phi}$, i.e. towards all things that are blue. Thus, by using a placeholder, the memory 
vectors for nouns will cluster together in one region of space, and the vectors for adjectives will cluster together in another region of space, and things that are colours will cluster separately from things that are coloured. This is a subtle distinction but Jones and Mewhort (2007) have shown it to be an important and powerful one.

\subsection{Retrieval}

There are two categories of information retrieval processes used in vector-symbolic models: unbinding, which retrieves information from a particular vector, and resonance, which allows information to be retrieved from the entire library of vectors in memory. Many-to-one models, such as TODAM (Murdock, 1982) only use unbinding. One-to-one models that do not use binding to encode associations, such as MINERVA (Hintzman, 1986), only use resonance. In many-to-many systems, these two retrieval processes are complementary. For example, resonance can be used to retrieve a vector, which can then be unbound.

Unbinding Consider a simple example where the agent is given a set of coloured shapes to remember: blue triangle, green square, red circle. In a many-to-one vector model this could be encoded by binding $(*)$ the vectors for the shapes to the colours, then summing to create a memory vector:

$$
\mathbf{m}=\mathbf{e}_{\text {blue }} * \mathbf{e}_{\text {triangle }}+\mathbf{e}_{\text {green }} * \mathbf{e}_{\text {square }}+\mathbf{e}_{\text {red }} * \mathbf{e}_{\text {circle }}
$$


The colour of any one of these shapes could then be recalled by unbinding (\#) using the shape to probe memory:

$$
\mathbf{e}_{\text {triangle }} \# \mathbf{m} \approx \mathbf{e}_{\text {blue }}
$$

In a many-to-many vector model, unbinding may use the placeholder as the probe. The placeholder is a special, randomly generated atomic vector that acts as a key to all of memory. The placeholder is initially used in binding:

$$
\begin{aligned}
& \mathbf{m}_{\text {blue }}=\boldsymbol{\Phi} * \mathbf{e}_{\text {triangle }} \\
& \mathbf{m}_{\text {triangle }}=\mathbf{e}_{\text {blue }} * \boldsymbol{\Phi}
\end{aligned}
$$

The placeholder can then be used in unbinding:

$$
\boldsymbol{\Phi} \# \mathbf{m}_{\text {triangle }} \approx \text { blue }
$$

Resonance (one-to-one) The term resonance comes from MINERVA (Hintzman, 1986), but it is implemented differently across different models. In MINERVA, the process of resonance begins by measuring the similarity (cosine) of each vector in memory to the probe. Then resonance computes a weighted sum of all vectors in memory. This sum, termed the echo, is what the model retrieves from memory. Each vector in the sum is weighted by its similarity to the probe raised to an exponent. 
For example, the three shapes might be represented as:

$$
\begin{aligned}
& \mathbf{m}_{\text {blue triangle }}=\mathbf{e}_{\text {blue }}+\mathbf{e}_{\text {triangle }} \\
& \mathbf{m}_{\text {green square }}=\mathbf{e}_{\text {green }}+\mathbf{e}_{\text {square }} \\
& \mathbf{m}_{\text {circle red }}=\mathbf{e}_{\text {red }}+\mathbf{e}_{\text {circle }}
\end{aligned}
$$

If the probe is triangle, then the echo would approximate:

$$
\begin{aligned}
& 0.5^{b} \mathbf{m}_{\text {blue triangle }}+0.0^{b} \mathbf{m}_{\text {green square }}+0.0^{b} \mathbf{m}_{\text {green square }} \\
& =0.5^{b}\left(\mathbf{e}_{\text {blue }}+\mathbf{e}_{\text {triangle }}\right)
\end{aligned}
$$

such that the memory system would remember that the triangle is blue. The exponent $b$ is a small, positive integer that is odd-numbered so as to preserve the sign of the similarity. Note that the similarity values of 0.5 and 0.0 are approximate. Random vectors in a high dimensional space have an expected cosine of 0 , but the actual cosine between any two random vectors will be a little more or a little less.

The exponent $b$ critically allows one-to-one vector models to function even when there is a very large amount of data in memory. If the exponent $b$ is 1 , the result of resonance roughly imitates decoding in a simple associative memory, such as a Hopfield network. With an exponent greater than one, resonance increases the signal to noise ratio in the echo by increasing the relative weighting of the memory vectors most similar to the probe. If $b$ is too low, a large number of partial matches in memory could easily overwhelm an exact match to the probe, 
resulting in a poor echo. With a high $b$, the echo will essentially just be the most similar vector in memory to the probe. In MINERVA, a $b$ of 3 is standardly used, but a $b$ of 3 may be too low when modelling a larger sum of knowledge than what is typically necessary to model a psychology experiment (e.g., in modelling word pronunciation, such as in Kwantes \& Mewhort, 1999).

In the Iterative Resonance Model (IRM; Mewhort \& Johns, 2005), resonance is iterated, and with each iteration $b$ is increased until a decision to stop iterating is made, resulting in either successful retrieval or a failure to retrieve. This approach has two benefits: (1) the number of iterations can be used to predict response time in memory tasks, and (2) it eliminates $b$ as a tweaking parameter by introducing a theory-driven approach to setting its value.

Resonance (many-to-many) Although the term resonance is used to describe retrieval in manyto-many vector models, the implementation is different and simpler: Essentially, the memory vector most similar to the probe is retrieved. This can be understood as a kind of spreading activation (Rutledge-Taylor et al., 2014). The probe and memory vectors can be understood as points on a hypersphere, such that the cosine measures the distance between them. One can imagine a ripple of activation spreading out from the probe across the surface of the hypersphere. The memory vectors closest to the probe become active in working memory, with the closer vectors becoming active sooner. This model of resonance allows BEAGLE (Jones \& Mewhort, 2007) to make semantic priming reaction time predictions (e.g., that doctor is recognized faster when preceded by nurse than when preceded by an unrelated prime such as stapler) and to model the fan-effect in DSHM (Rutledge-Taylor et al., 2014). 


\subsection{Conclusions}

We hold that, in order to bridge the gap between human experience and neural connectivity, explanations at both the symbolic and sub-symbolic levels of description are necessary parts of theory in cognitive science. As we have illustrated in this chapter and the previous two chapters, cognitive models that use vector-symbolic architectures intrinsically operate at both of these levels of description and thereby provide a needed bridge between the two kinds of explanation.

At the sub-symbolic level is the vector-symbolic architecture itself, and the linear algebra operations on vectors that comprise the architecture: similarity, superposition, binding, unbinding, permuting, un-permuting. All of these operations are easily amenable to neural implementation, as in the NEF (Eliasmith, 2013).

At the symbolic level, we have the cognitive model itself, and the cognitive processes that define it. On the basis of their storage and retrieval mechanisms, we classify existing vectorsymbolic cognitive models into many-to-one, one-to-one, and many-to-many vector models. This classification scheme highlights stark differences between these models.

Many-to-many vector models differ from the other two classes of model in two important ways. First, many-to-many models use a placeholder vector to stand for "this item I am thinking about". The placeholder acts as a symbol with an important functional role but no perceptual or conceptual meaning. It may be useful to incorporate other kinds of function vectors in future models, e.g., a wildcard vector to stand for "that item that I'm not thinking about", vectors to stand for emotional states, for positive and negative valence (see Chapter 8), for truth values, et cetera. 
Secondly, in many-to-many models, memory vectors stand for particular concepts, whereas in one-to-one models concepts are an emergent phenomenon produced by the echoes retrieved using resonance (Hintzman, 1986). While the conceptual representations in many-tomany models are powerful, having a predefined number of concepts is implausible and limiting.

We agree with Hintzman (1986) that concepts are an emergent property of retrieval. As we argue in the next chapter, the memory vectors that stand for concepts in many-to-many vector models are, in fact, the echoes in one-to-one vector models. But using a one-to-one model to do the kind of large scale modelling in many-to-many models is impossible because one-to-one models store all experiences without any form of compression. However, a neural implementation of a one-to-one model would naturally be lossy in its storage, and so could provide a plausible account of concept formation over a lifetime of experiences.

Many-to-one vector models can be constructed in the Neural Engineering Framework (Eliasmith, 2013) as self-recurrent neural groups. By contrast, one-to-one and many-to-many models as yet lack a neural explanation. Finding a means of translating one-to-one and many-tomany vector models into neural models may provide a route to a unified, vector-symbolic account of memory storage and retrieval. As we noted earlier, a one-to-one model behaves somewhat like a Hopfield network when the resonance exponent $b$ is set to 1 . To implement a one-to-one model as a network, one needs to find a mechanism analogous to $b$ that can act to increase the signal to noise ratio in the echo.

Unification in other areas, such as representation, is important too. Incorporating a vector-symbolic model of string encoding (Hannagan et al., 2011) into the BEAGLE model of 
semantics (Jones \& Mewhort, 2007) could, for instance, allow BEAGLE to model how shared orthography can help and hinder in understanding the meaning of words.

Eventually, we hope to see developed a vector-symbolic cognitive architecture, which not only presents a unified and neurally plausible approach to representation, storage, and retrieval, but also extends the vector-symbolic account beyond its roots in memory theory, and integrating it into accounts of emotions, attention, perception, and consciousness. As cognitive scientists, it is important to keep in mind our ultimate, lofty, and collective goal of a theory that unifies not only all aspects of the cognition, but all relevant levels of description. 


\section{Chapter 5 The Memory Tesseract: Distributed MINERVA}

Computational memory models can explain the behaviour of human memory in diverse experimental paradigms. But research has produced a profusion of competing models, and, as different models focus on different phenomena, there is no best model. However, by examining commonalities between models we can move toward theoretical unification. Computational memory models can be grouped into composite and separate storage models. We prove that MINERVA 2, a separate storage model of long-term memory, is mathematically equivalent to composite storage memory implemented as a fourth order tensor, and approximately equivalent to a fourth-order tensor compressed into a holographic vector. Building of these demonstrations, we show that MINERVA 2 and related separate storage models can be implemented in neurons. Our work clarifies the relationship between MINERVA 2 and other memory models, and thereby moves memory models a step closer to theoretical unification.

\subsection{Introduction}

In what follows, we argue that the class of memory models that use high dimensional vectors can be understood as belonging to a single mathematical framework. These memory models include the MINERVA 2 (Hintzman, 1984) model and variants (e.g., Dougherty, Gettys, \& Ogden, 1999; Jamieson, Crump, \& Hannah, 2012; Jamieson \& Mewhort, 2011; Kwantes, 2005; Thomas, Dougherty, Sprenger, \& Harbison, 2008), composite vector models (e.g., Anderson, 1973; Johns, Jones, \& Mewhort, 2012; Murdock, 1989), matrix models (e.g., Farrell \& Lewandowsky, 2002; Humphreys, Pike, Bain, \& Tehan, 1989b; Howard \& Kahana, 2002; Lewandowsky \& Farrell, 
2008), tensor models (e.g., Humphreys, Bain, \& Pike, 1989a; Osth and Dennis, 2015;

Smolensky, 1990), holographic models of short-term and long-term memory (e.g., Eich, 1982;

Franklin \& Mewhort, 2015; Murdock, 1993), the Generalized Context Model of categorization

(GCM; Nosofsky, 1986, 1991), the BEAGLE model of distributional semantics (Jones \&

Mewhort, 2007), and variants of BEAGLE, such as the DSHM (Rutledge-Taylor, Kelly, West, \& Pyke, 2014) and HDM (Kelly, Kwok, \& West, 2015) models of memory.

In particular, we examine the relationship these models have to MINERVA 2 (Hintzman, 1984), a computational model of human memory intended to describe long-term memory (both episodic and semantic). MINERVA 2 has been applied to a number of experimental paradigms, including judgement of frequency tasks (Hintzman 1984), recognition tasks (Hintzman, 1984), "schema-abstraction" or category learning (Hintzman, 1984; 1986), implicit learning tasks such as artificial grammar learning (Jamieson \& Mewhort, 2009; 2011), the production effect (Jamieson, Mewhort, \& Hockley, 2016) as well as speech perception (Goldinger, 1998), and naming words from print (Kwantes \& Mewhort, 1999).

Variations on the MINERVA 2 model address an even broader range of phenomena. MINERVA-AL makes and corrects predictions to capture numerous associative learning phenomena from both the animal and human learning literature (Jamieson et al., 2012). Kwantes (2005) used a MINERVA variant to study how semantic similarity can be learned from word cooccurrence in the language. Johns, Jamieson, Crump, Jones, and Mewhort (2016) use a MINERVA variant to model the production of natural language syntax given sentence exemplars. MINERVA-DM models judgements of likelihood to account for heuristics and biases in decision-making (Dougherty et al., 1999). The HyGene model (Thomas et al. 2008) is an 
extension of MINERVA-DM to hypothesis generation and accounts for how errors in hypothesis generation lead to errors in judgement and decision-making.

We will use the term MINERVA 2 to refer specifically to Hintzman's model, and MINERVA to refer collectively to MINERVA 2 and any model based on it.

In this chapter, we begin by providing an introduction to the MINERVA 2 memory model. Then, we compare the various memory models that use high-dimensional vectors. We note that the structure of the memory models vary according to the demands of the experimental tasks they are designed for. If the aim of research in memory is to develop a single, unified model of human memory, then there is a need for a model that has a consistent structure across tasks.

The number of representations that a memory model associates together (one, two, three, etc.) dictates the structure of the model. A composite vector model (e.g., Anderson, 1973; Johns et al., 2012; Murdock, 1989) does not associate representations together (i.e., the model stores singleton items). A matrix model associates together pairs of representations (e.g., Farrell \& Lewandowsky, 2002; Howard \& Kahana, 2002; Humphreys et al., 1989b). A third-order tensor associates triples of representations (e.g., Humphreys et al., 1989a; Osth \& Dennis, 2015). More generally, a tensor of order $k$ stores associations of $k$ representations (Smolensky, 1990). As the number of representations associated together increases, the tensor of that association becomes exponentially larger, and the computational demands also increase exponentially.

Holographic vector models (e.g., Eich, 1982; Franklin \& Mewhort, 2015; Murdock, 1993 ) provide a solution. Holographic vectors can store associations of an arbitrary number of 
items in a vector of fixed dimensionality (Plate, 1995). Thus, holographic vectors address both the problem of growing computational demands and variations in model structure across tasks.

However, because of the way holographic vectors compress associations into a vector, holographic vectors add considerable noise to memory retrieval operations. One solution to the problem of noise is to embed holographic vectors into a MINERVA model, as in the Holographic Exemplar Model (Jamieson \& Mewhort, 2011). By combining holographic vectors with the MINERVA architecture, the Holographic Exemplar Model creates a system that can store arbitrarily complex associations and retrieve them with fidelity.

If the aim of memory research is to develop a unified model of memory, able to explain behaviour on a wide range of memory tasks, something like the Holographic Exemplar Model seems like a good basis. The literature demonstrates that the MINERVA architecture can already account for a wide variety of tasks. Combining MINERVA with holographic vectors allows the Holographic Exemplar Model to flexibly represent the different information structures of different tasks.

However, MINERVA has several limitations. One limitation is that it is not clear that MINERVA can be realized in neurons without making implausible assumptions. Another limitation is that MINERVA scales poorly to large-scale tasks. The model grows linearly in scale with the number of experiences it stores in memory. Large-scale MINERVA-like models (e.g., Jones and Mewhort, 2007; Kwantes \& Mewhort, 1999; Kwantes, 2005; Rutledge-Taylor et al., 2014) modify the model's assumptions in order to scale to large tasks. This threatens the validity of MINERVA as a theory of both episodic and semantic memory. According to the MINERVA theory, semantic memories are not a separate memory system but are the result of aggregate 
retrieval across many episodes. That MINERVA needs to be modified to accommodate largescale tasks means that there is a de facto separation of semantic and episodic memory models in the literature.

To address this issue, we present a proof of exact mathematical equivalence between MINERVA and an auto-associative fourth-order tensor memory, which we refer to as a "memory tesseract" as it is a matrix with four equal dimensions. Implemented as a fourth-order tensor, MINERVA is invariant in scale with respect to the number of experiences it has had, allowing the model to be scaled up to arbitrarily large tasks.

In what follows, we discuss the implications of this equivalence in detail. One implication is that MINERVA is not different in kind from composite vector, matrix, tensor, and holographic vector memories. Unfortunately, fourth-order tensors are very large, which is one reason why holographic vectors are such an appealing alternative to tensors. Implementing MINERVA as a fourth-order tensor is impractical except when simulating very large-scale memory and learning tasks.

However, because a fourth-order tensor can be compressed into a holographic vector, we can compress MINERVA into a holographic vector to create a noisy approximation to MINERVA. But we return to our earlier problem: holographic vectors introduce a great deal of noise. Levy and Gayler (2009) provide a solution: by averaging across many holographic vectors, the noise can be eliminated. We present a proof of approximate equivalence between MINERVA and a modification of the model proposed by Levy and Gayler (2009).

We show that MINERVA 2, understood as the holographic approximation to the memory tesseract, can be built out of realistic, simulated neurons using the Neural Engineering 
Framework (Eliasmith, 2013). While we do not have evidence that a structure like this exists in the brain, we show that it is, at least, in principle, possible for neurons to implement the type of memory system we have described.

To illustrate the behaviour of the memory models, we present a set of simulations of the models on artificial data. Models included in the simulations are MINERVA 2, the memory tesseract, the holographic approximation to the memory tesseract based on Levy and Gayler's model, a holographic vector model, and a matrix model.

We also compare performance of the holographic approximation to Johns et al.'s (2016) MINERVA model of a sentence production task. We find that the holographic approximation provides a means of implementing MINERVA as a memory system that is invariant in scale with respect to the number of experiences stored, but does so at the cost of increased noise from the compression of the memory traces into a smaller data structure. Additionally, to be feasibly implemented on very large-scale tasks, the holographic approximation should be simulated on a highly parallel computer, such as a neuromorphic computer.

This work clarifies the relationship between MINERVA and other memory models that use high dimensional vectors. This work serves to demonstrate that MINERVA can potentially be used as a basis for unifying high dimensional vector memory modelling, that MINERVA is scalable to arbitrarily long-term learning if implemented on a massively parallel computer, and that MINERVA can be plausibly realized in neurons. This work clarifies the relationship between MINERVA and other memory models that use high dimensional vectors. This work serves to demonstrate that MINERVA can potentially be used as a basis for unifying high dimensional 
vector memory modelling, that MINERVA is scalable to arbitrarily long-term learning, and that MINERVA can be plausible realized in neurons.

\subsection{How does MINERVA work?}

In MINERVA, each individual experience, or episode, is represented by a high dimensional vector, a list of features represented by numerical values. Memory is a table where each row is a vector representing an episode trace corresponding to a stored experience. Each new experience is stored as a new row in the memory table. New experiences do not need to be novel. A repeated experience is also stored as a new row, separate from previous instances of that experience.

In MINERVA, memory retrieval is not a look-up process, it is a reconstruction process. In the words of Tulving and Watkins (1973, p. 744), a retrieval cue "combines or interacts with the stored information to create the memory of a previously experienced event”. When a retrieval cue is presented, each vector in the table "resonates" with the cue in proportion to its similarity to the cue (Hintzman, 1986).

Similarity is computed as a normalized dot-product of the cue's vector with the stored vector. Each stored vector is activated by its cubed similarity to the cue. Information is retrieved from memory in the form of a new vector, called an echo. The echo is a weighted sum of the vectors in the table, each vector weighted by its activation. By computing activation as the cube of similarity, the contribution of the most similar vectors (or experiences) is emphasized and that of the least similar (and least relevant) is minimized. The echo is used by the model to respond to the cue as appropriate for a task. Abstract, conceptual, semantic, and categorical information reflect aggregate retrieval over many experiences (e.g., Goldinger, 1998; Kwantes, 2005). 
Hintzman (1986, p. 411) summarizes MINERVA 2's key assumptions:

(1) only episode traces are stored in memory,

(2) repetition produces multiple traces of an item,

(3) a retrieval cue contacts all traces simultaneously,

(4) each trace is activated according to similarity to the cue,

(5) all traces respond in parallel, retrieved information reflects their summed output.

According to Hintzman (1990), MINERVA 2 can be understood as an artificial neural network (see Figure 5.1). A layer of input nodes represent the cue, a layer of output nodes represent the echo, and between the two is a hidden layer of nodes. In the hidden layer, each node corresponds to an episode trace. It follows that MINERVA's hidden layer is a localist network: specific nodes represent specific pieces of information.

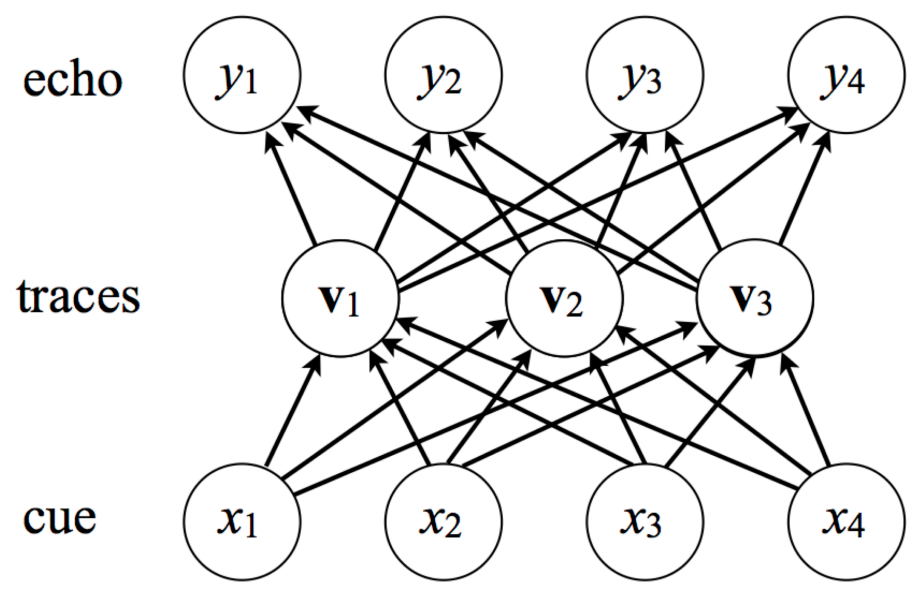

Figure 5.1. MINERVA represented as a neural network that operates on four-dimensional vectors $(n=4)$ and has three memory traces stored $(m=3)$. Given a cue $\mathbf{x}$, the network retrieves an echo $\mathbf{y}$, a weighted sum of the traces $\mathbf{v}_{1}, \mathbf{v}_{2}$, and $\mathbf{v}_{3}$. The input and output layers can alternatively be represented by a single input/output layer with recurrent connections from the hidden layer, as in Hintzman (1990). 
Modellers using MINERVA are generally agnostic as to how the model is related to the brain. No one claims that for each new experience one grows a new neuron that is forever singly dedicated to that particular experience. But no other interpretation of how MINERVA can be implemented in neurons has been previously proposed, leaving open the question of MINERVA's neural plausibility.

\subsection{A comparison of memory models}

The memory models discussed here use vector and tensor representations to simulate the processes of storage and retrieval. Tensors are a generalization of matrices. A vector is a first order tensor, a matrix is a second order tensor, a third order tensor is a "3D matrix" or a stack of matrices, and we use the term tesseract to refer to a fourth order tensor or "4D matrix."

For our purposes, vector-based memory models can be divided into five categories: composite vector memory (Anderson, 1973; Murdock, 1989; Johns et al., 2012), where all memories are stored in a single vector; matrix memory (e.g., Farrell \& Lewandowsky, 2002; Humphreys et al. 1989b; Howard \& Kahana, 2002; Lewandowsky \& Farrell, 2008), where all memories are stored in a single matrix; tensor memory (e.g., Humphreys et al., 1989a; Osth \& Dennis, 2015; Smolensky, 1990), where all memories are stored in a single, higher-order tensor (e.g., a "3D" or “4D” matrix); holographic vector memory, which compress tensors into a vector (e.g., Eich, 1982; Franklin \& Mewhort, 2015; Murdock, 1993); and multi-vector memory, where multiple vectors are used to store memories (e.g., MINERVA; Hintzman, 1984; REM; Shiffrin \& Steyvers, 1997; BEAGLE; Jones \& Mewhort, 2007; and variants; Kelly et al., 2015; RutledgeTaylor et al., 2014). Composite vector, matrix, tensor, and holographic vector memory are all 
examples of what Clark and Gronlund (1996) refer to as composite or distributed storage models because all stored experiences are represented as distributed across a shared set of units. Conversely, multi-vector memory is what Clark and Gronlund refer to as a separate storage model because different experiences are represented in different sets of units.

Humphreys et al. (1989b) note that their matrix memory, MINERVA 2, and the holographic vector model TODAM (Murdock, 1993) retrieve information as a sum of all traces in memory, each trace weighted by its similarity to the cue. As we will show, these models are not different in kind, and so we elect to use the term echo, normally reserved for MINERVA, to refer to the retrieved vector in all of the memory models we discuss in this chapter. We will use the term probe to refer to the vector used as a retrieval cue.

In vector-based memory models, a to-be-remembered item is represented as a vector. These vectors are typically high dimensional with randomly generated values. For example, in the simulations illustrated in Figures 5.2, 5.3, and 5.6, we use vectors of 64 dimensions. The 64 values for each vector are randomly sampled from a normal distribution.

Here we compare composite vector, matrix, tensor, holographic vector, and multi-vector models of memory.

\subsection{Composite vector models}

A composite vector memory is simple: it is the sum of the vectors that represent the to-beremembered items. To store an item in memory, the vector representing that item is weighted by how well it is encoded and then added to the memory vector. The familiarity of a given item is 
the dot product of that item with the memory vector. Anderson (1973), Murdock (1989), and Johns et al. (2012) use composite vector models to account for performance on recognition tasks.

Unlike the other models we discuss, composite vector memories store items individually. Composite vector memories do not store associations between items. Unlike the other models, a composite vector memory does not retrieve an echo given a probe. Rather, the memory vector itself is a linear combination of item vectors, like the echoes retrieved by the more complicated memory models.

\subsection{Matrix and tensor models}

A key point of comparison is how vector-based models represent associations between items. Using the Hebbian learning rule, for a pair of items, each represented as a vector of features, an association between that pair of items is represented by the matrix outer-product of the vectors. Smolensky (1990) notes that the tensor product, a generalization of the matrix product, can be used to form associations between an arbitrary number of items (pairs, triples, quadruples, etc.), though at the cost of producing progressively larger and more unwieldy tensors.

The order of the tensor used to store memories indicates the number of vectors the memory model associates together. Items that are not associated can be represented in a composite vector memory, which is a first order tensor. An association between a pair of items is represented as the tensor product of the items' vectors, which is a second order tensor, or matrix.

A matrix memory is the sum of such associations between pairs of vectors. Howard and Kahana's (2002) matrix memory is context $\mathrm{x}$ item; it associates a vector representing an item with a vector representing a context. Humphreys et al.'s (1989b) matrix memory is item a x item 
b; it associates two different items together. Farrell and Lewandowky's (2002) matrix memory, the SOB model, is item a $\mathrm{x}$ item $\mathbf{a}$; it associates an item with itself. The SOB model is thus autoassociative whereas the Howard and Kahana (2002) and Humphreys et al. (1989b) models are hetero-associative. Lewandowky and Farrel's (2008) C-SOB model is both, consisting of two matrices: one is item a $\mathrm{x}$ item a and the other is context $\mathrm{x}$ item, allowing the model to associate an item with itself, but also with a context.

If three items are being associated, the result is a "3D matrix", or third order tensor. Humphreys et al.'s (1989a) third order tensor memory is context $\mathbf{x}$ item a x item b, that is, it associates two different items together with a representation of context. Building on Humphreys et al.'s model, Osth and Dennis (2015) use a context $\mathrm{x}$ item a $\mathrm{x}$ item $\mathbf{b}$ third order tensor to model memory for pairs of items and a context $\mathrm{x}$ item matrix for memory of individual items. Osth and Dennis note that the two memories they use, one for individual items and one for pairs, are separate strictly due to mathematical necessity. Osth and Dennis are not committed to pairs and singletons of items having distinct neural substrates or memory systems.

There is a problem here. Across these models, the architecture of memory is being modified to suit the particulars of the tasks being modelled. If we are testing for the familiarity of an item, we can use a composite vector memory. If we need a cue (be it an item or context), we use a matrix memory. If we use two cues (be it an item and context, or two items) we use a third order tensor. But what if we need to use three cues? Do we then need to use a fourth order tensor? What about four cues? Using this approach, not only does the architecture of memory need to be changed depending on the particulars of the task, but also the architecture becomes increasingly unwieldy as the task becomes more complex. 


\subsection{Holographic vector models}

Given $k$ vectors, each of $n$ dimensions, an association between those vectors can be represented as the tensor product of the vectors, which is a tensor of $n^{k}$ values (Smolensky, 1990). A tensor is a potentially unwieldy representation if $k$ is large. Furthermore, using tensors necessitates a separate memory store for each value of $k$ because tensors of different orders cannot be added together (e.g., one cannot add together a vector and a matrix).

Conversely, in a holographic vector memory, an association between a set of items is the convolution of the vectors representing those items. If circular convolution is used instead of the tensor product, the association of $k$ vectors of $n$ dimensions is itself a vector of $n$ dimensions, irrespective of $k$ (Plate, 1995).

Holographic vectors can represent arbitrarily complex associations of items and context. Using holographic vectors makes it unnecessary to use matrices or higher order tensors to represent associations and allows modellers to adopt a memory architecture that is compact and invariant with respect to the complexity of the associations.

Holographic vectors are part of a family of related computational memory systems called vector-symbolic architectures (Gayler, 2003) that also includes MAP codes (Gayler, 2003), square matrix representations (Kelly, 2010) and binary spatter codes (Kanerva, 1996; see Kelly, Blostein, \& Mewhort, 2013 for a review). All vector-symbolic architectures can be understood as compressing the tensor product of a set of item vectors into a memory vector that compactly represents the association between those items. As holographic vectors are the most commonly used vector-symbolic architecture in the literature, we restrict our discussion to holographic 
vectors, and in particular, holographic reduced representations (Plate, 1995), which use circular convolution to encode associations. As Vector-Symbolic Architectures all have similar properties, much of our discussion thus still pertains to all memory models that use VectorSymbolic Architectures.

Cognitive models of memory that use holographic vectors can explain and predict a variety of human memory phenomena (e.g., Eich, 1982; Franklin \& Mewhort, 2015; Murdock, 1993). The majority of models that use holographic vectors are purely cognitive and, as such, do not concern themselves with the question of how the models could be implemented in the brain. However, the Neural Engineering Framework (Eliasmith, 2013) uses holographic vectors as the representation scheme for their neurocognitive model of the brain (Eliasmith et al., 2012). Thagard and Stewart (2011) suggest that holographic vectors are the neural underpinning of creativity. Holographic vectors present a plausible means by which the brain could recursively combine patterns of neural activations representing simple concepts to generate new patterns of activation that represent novel, complicated concepts.

However, circular convolution is a lossy compression of the tensor-product (Plate, 1995; for discussion see Kelly et al., 2013). Information is lost in the act of compressing a tensor of size $n^{k}$ down to a vector of size $n$.

Holographic vector models differ from matrix and tensor models in that:

(1) the lossy compression of the tensor to a vector adds noise to the echo,

(2) individual items and associations between sets of any size (singles, pairs, triples, quadruples, etc.) can all be stored together as a sum in a single vector memory, and 
(3) convolution is commutative whereas the matrix or tensor product are noncommutative.

With regard to (3), that convolution is commutative means that $\mathbf{a} * \mathbf{b}=\mathbf{b} * \mathbf{a}$, for any pair of vectors $\mathbf{a}$ and $\mathbf{b}$, where * denotes convolution. The matrix or tensor product are noncommutative, $\mathbf{a} \mathbf{b}^{\mathrm{T}} \neq \mathbf{b} \mathbf{a}^{\mathrm{T}}$. A consequence of this distinction is that a memory model that encodes $\mathbf{a} * \mathbf{b}$ to represent an association of $\mathbf{a}$ with $\mathbf{b}$ has also encoded an association of $\mathbf{b}$ with $\mathbf{a}$, that is to say, the association is symmetric. Whereas a memory model that encodes $\mathbf{a} \mathbf{b}^{\mathrm{T}}$ has encoded an association of $\mathbf{a}$ with $\mathbf{b}$ without encoding the association of $\mathbf{b}$ with $\mathbf{a}$, such that if the model is given $\mathbf{a}$ as a probe the echo will resemble $\mathbf{b}$, whereas if it is given $\mathbf{b}$ as a probe, the echo will not necessarily resemble a.

The decision to model associations between items in memory as symmetric or asymmetric will have consequences for the model's behaviour. However, the decision to use a commutative or non-commutative mathematical operation to represent associations can be made completely independently of the decision to use symmetric or asymmetric associations. The matrix or tensor product can be used to encode symmetric associations by always adding both a $\mathbf{b}^{\mathrm{T}}$ and $\mathbf{b} \mathbf{a}^{\mathrm{T}}$ to memory, for any associated pair of items a and $\mathbf{b}$. Conversely, convolution can be used to encode asymmetric associations by encoding an association of an item a with an item $\mathbf{b}$ as $(\mathbf{P} \mathbf{a}) * \mathbf{b}$, such that $(\mathbf{P} \mathbf{a}) * \mathbf{b} \neq(\mathbf{P} \mathbf{b}) * \mathbf{a}$, where $\mathbf{P}$ is the permutation matrix of a random permutation (Plate, 1995).

As the distinction between commutative and non-commutative operations for encoding associations is immaterial, the loss of information from the compression is the only reason one might prefer the aforementioned matrix or tensor memories to a holographic memory. However, 
combining holographic vectors with MINERVA creates a system that can store arbitrarily complex associations between items and contexts, and retrieve them with fidelity (Jamieson \& Mewhort, 2011), providing a remedy for the holographic vector information loss problem.

\subsection{Multi-vector models}

In multi-vector memory models, memories are stored as a collection of vectors in a table. Multivector models include MINERVA models, the Retrieving Effectively from Memory model (REM; Shiffrin \& Steyvers, 1997) the Generalized Context Model of categorization (GCM; Nosofsky, 1986, 1991), the BEAGLE model of distributional semantics (Jones \& Mewhort, 2007), and variants of BEAGLE, such as the DSHM (Rutledge-Taylor, Kelly, West, \& Pyke, 2014) and HDM (Kelly, Kwok, \& West, 2015) models of memory.

MINERVA and GCM store each experience as a separate vector, such that the number of vectors grows with each additional memory trace stored. Conversely, DSHM, HDM, and BEAGLE store each distinct concept as a separate vector, such that the number of vectors grows only with the addition of new concepts. REM takes a hybrid approach and stores vectors for both individual experiences and individual concepts. REM makes the argument that concepts are frequently revisited experiences, such that vectors in memory exist on a continuum from experience to concept. Unlike REM, MINERVA holds that concepts or categories are emergent from aggregate retrieval across experiences and as such do not require a distinct storage mechanism.

In both MINERVA and GCM, when a probe is presented to memory, each vector in memory is activated according to its similarity to the probe raised to some power. In MINERVA 
2 , similarity is measured by a normalized dot product, which is a measure of the angle between two vectors and ranges from +1 to -1 . The GCM calculates similarity as $e$ to the power of the negative Euclidean distance, which is 1 when the vectors are identical and asymptotically approaches 0 as the vectors move further apart. As similarity in the GCM is always positive, the GCM can raise similarity to even numbered powers without losing the sign of the similarity. In the GCM, similarity is raised to the power of $c$, where $c$ is a sensitivity parameter set by the modeller. Conversely, in MINERVA 2, the exponent is always 3, though some variants of MINERVA dynamically vary the exponent (e.g., Mewhort \& Johns, 2005) or use a larger exponent to minimize noise (e.g., Johns et al., 2016).

The GCM differs from MINERVA in that the GCM is a model of categorization judgements whereas MINERVA is a more general model of memory. MINERVA retrieves from memory an echo, a vector representing an inexact recollection. Conversely, the GCM retrieves from memory the amount of evidence for each possible categorization, computed as the sum of the activations of the exemplars of each category.

MINERVA 2 can be used as a model of category learning (e.g., Hintzman, 1986) and can be made to imitate the GCM. MINERVA 2 can store exemplar-category pairs by concatenating a vector representing an exemplar with a vector representing the corresponding category. Given a probe, MINERVA 2 retrieves an echo that is a sum of the traces in memory, each weighted by their similarity to the probe. The latter half of the echo will be a weighted sum of category vectors. The cosine between the latter half of the echo and each category vector will be the amount of evidence for each category, as in the GCM. 
The REM model (Shiffrin \& Steyvers, 1997) borrows aspects of MINERVA 2. Like MINERVA, a probe contacts all vectors in memory in parallel. Like MINERVA, REM computes a match between a probe and each vector in memory. Unlike MINERVA, the activation of a vector is computed as a likelihood ratio: the probability of the observed similarity given that the probe and the trace are a match divided by the probability of the similarity given that the probe and trace are a mismatch. Computing activation using Bayesian probability allows REM to model a number of list strength effects (Shiffrin \& Steyvers, 1997) that are problematic for MINERA and composite memory models (see Shiffrin, Ratcliff, \& Clark, 1990 for a discussion), though more recent work has suggested alternate approaches to accounting for list strength effects in composite (Johns et al., 2012) and MINERVA models (Jamieson et al., 2016).

BEAGLE (Jones \& Mewhort, 2007) and variants (Rutledge-Taylor et al., 2014; Kelly et al., 2015) are collections of holographic vectors. For each item in the model, there is one holographic vector that is constructed as a sum of all experiences with that item. BEAGLE and variants differ from MINERVA in that these models have only one memory vector per item, such that each item is represented in memory by an aggregate of all experiences with that item.

Another difference between the MINERVA and BEAGLE models is how information is retrieved from memory. BEAGLE is a model of distributional semantics rather than memory per se, and as such, does not have a well-defined retrieval mechanism. Inspired by BEAGLE's account of semantic priming (Jones, Kintsch, \& Mewhort, 2006), the DSHM (Rutledge-Taylor et al., 2014) and HDM (Kelly et al., 2015) memory models retrieve from memory the vector most similar to the probe and compute reaction time as an inverse function of similarity. Conversely, 
in MINERVA, rather than retrieving the vector most similar to the probe, MINERVA retrieves an echo, a sum of all vectors in memory weighted by their similarity to the probe.

The memory vectors stored in BEAGLE, DSHM and HDM can be thought of as analogous to the echoes retrieved from memory by MINERVA. A MINERVA model that uses the MINERVA 2 architecture and the representational assumptions of BEAGLE can generate echoes that closely approximate the memory vectors in BEAGLE.

In BEAGLE and variants, an item is represented by two vectors: an environment vector $\mathbf{e}_{\text {item }}$ that stands for the perceptual characteristics of the item and a memory vector $\mathbf{m}_{\text {item }}$ that stores an aggregate of all experiences with that item. Each individual experience of an item can be represented by a vector $\mathbf{v}_{i}$, for $i=1$ to $m$, where $m$ is the number of instances in the data set (the number of words in a corpus for BEAGLE or the number of stimuli in the experiment for HDM or DSHM). Each $\mathbf{v}_{i}$ is a holographic vector constructed as a sum of convolutions of environment vectors that co-occur with the item in instance $i$. A memory vector is a sum of those instances, $\mathbf{m}_{\text {item }}=\Sigma \mathbf{v}_{i}$ for all $i$ from 1 to $m$ where instance $i$ is an instance of the item.

A MINERVA model can mimic BEAGLE by storing $\mathbf{e}_{i} \mid \mathbf{v}_{i}$ pairs, where $\mid$ indicates concatenation of vectors. If $\mathbf{e}_{i}$ and $\mathbf{v}_{i}$ have a dimensionality of $n$, the concatenated vector $\mathbf{e}_{i} \mid \mathbf{v}_{i}$ has a dimensionality of $2 n$. Given an item as a cue, we can retrieve an echo that approximates a BEAGLE memory vector. Given the probe $\mathbf{e}_{\text {item }} \mid \mathbf{z}$, where $\mathbf{z}$ is a vector of zeros, the model will retrieve an echo $\mathbf{y}_{\text {item, }}$

$$
\mathbf{y}_{\text {item }}=\left(\left(\mathbf{e}_{\text {item }} \mid \mathbf{z}\right) \cdot\left(\mathbf{e}_{1} \mid \mathbf{v}_{1}\right)\right)^{3}\left(\mathbf{e}_{1} \mid \mathbf{v}_{1}\right)+\ldots+\left(\left(\mathbf{e}_{\text {item }} \mid \mathbf{z}\right) \cdot\left(\mathbf{e}_{m} \mid \mathbf{v}_{m}\right)\right)^{3}\left(\mathbf{e}_{m} \mid \mathbf{v}_{m}\right)
$$

If each environment vector $\mathbf{e}_{\text {item }}$ has a Euclidean length of one and is exactly orthogonal to all environment vectors for different items, then the latter half of the echo is exactly a memory 
vector in BEAGLE,

$$
\mathbf{y}_{\text {item }}=\Sigma \mathbf{e}_{i}\left|\mathbf{v}_{i}=\mathbf{e}_{\text {item }}\right| \mathbf{m}_{\text {item }}
$$

for all $i$ from 1 to $m$ where instance $i$ is an instance of the item. If the environment vectors are approximately orthogonal, as they are in BEAGLE, DSHM, and HDM, then the latter half of

$\mathbf{y}_{\text {item }}$ is approximately equal to $\mathbf{m}_{\text {item }}$ due to the introduction of zero mean noise from other traces in memory.

Thus, the GCM model of categorization, the BEAGLE model of distributional semantics, and the DSHM and HDM memory models based on BEAGLE, can all be instantiated within the MINERVA architecture and are compatible with MINERVA theory. However, in practical terms, the BEAGLE model cannot be implemented as a MINERVA model as described, as it would require storing one vector in the memory table for each word in the corpus. The size of a corpus may range from a few million words, to 100 million words (e.g., the British National Corpus), to 1.9 billion words (e.g., Global Web-Based English). Thus, a strict MINERVA instantiation of BEAGLE is not feasibly computable.

\subsection{MINERVA versus vector and matrix models}

MINERVA 2 differs from vector or matrix models in that:

(1) MINERVA 2 associates items by either adding together or concatenating the vectors representing those items, rather than using the tensor-product or convolution.

(2) All traces in the echo of MINERVA 2 are weighted by a normalized dot product rather than by the dot product. 
(3) All traces in the echo of MINERVA 2 are weighted by the cubed similarity to the cue. In a vector or matrix model, the similarity is not raised to an exponent.

The first difference, the use of addition or concatenation to form associations, is not essential. The Holographic Exemplar Model (Jamieson \& Mewhort, 2011) is a MINERVA that uses circular convolution rather than concatenation and thus gains the ability to represent arbitrarily complex associations using vectors of fixed dimensionality. The second difference, that MINERVA uses a normalized dot product, can be addressed by using normalization in vector or matrix models, as we will discuss in the Similarity in MINERVA section.

The third difference is critical. Raising similarity to an exponent of 3 sets the MINERVA models apart from the vector and matrix models. The purpose of this exponent is to make MINERVA nonlinear, as Hintzman explains (1990, p. 116):

This model escapes being just a less efficient version of the vector model by using nonlinearity. In particular, the activation of each hidden unit is a positively accelerated function of its match to the input vector, limiting the number of units that will respond significantly to any input, and thereby reducing noise.

By weighting each episode trace by the cube of its similarity, the traces that are most similar to the cue contribute much more to the echo than traces that have only partial similarity to the cue, or traces that have tiny, incidental similarity to the cue. Cubing similarity weights retrieval in favour of an exact match to a single item in memory over partial matches to several items in memory or slight matches with a very large number of items in memory.

Raising the similarity to any exponent larger than 1 would introduce non-linearity. However, when using even numbered exponents, the sign of the similarity is lost. MINERVA 2 
uses a normalized dot product to measure similarity, which ranges from +1 to -1 . To preserve the sign of the similarity, the MINERVA 2 model uses an exponent 3 rather than 2. Larger odd numbered exponents $(5,7,9, \ldots)$ also preserve the sign of the similarity and can be used to further reduce the amount of noise in the echo (e.g., Johns et al., 2016).

Non-linearity also allows MINERVA to "clean-up" the echo using iterative retrieval (Hintzman, 1986). MINERVA can "clean up" an echo by using the echo as a cue to produce a new echo. With each pass through the memory system, the contribution of the most similar episode traces grows. This process can be repeated until the echo reaches a steady state where it no longer changes, at which point the echo will closely resemble the trace in memory most similar to the cue (as illustrated in Figure 5.2).

The clean-up process also serves as a possible explanation for why we are faster to remember some things than others: echoes formed from frequently occurring and distinctive episode traces reach a steady state more quickly. For example, the number of iterations until the echo reaches a steady state is used to produce response latency predictions in the SOB (Farrell \& Lewandowsky, 2002) and C-SOB (Lewandowsky \& Farrell, 2008) models of memory.

Linear systems require an external clean-up memory. Unless some form of non-linearity is introduced, the echo produced by a holographic vector memory (e.g., TODAM; Murdock, 1993) or, to a lesser extent, a matrix memory (e.g., Humphreys et al., 1989b) is noisy (see Figure 5.2 for illustration) and requires an external memory for items in order to identify which item the echo most resembles (Murdock, 1993).

Raising similarity to an exponent is not the only way to introduce non-linearity. For example, non-linearity can be introduced by passing the output of a network through an 
activation function, such as a binary step function that casts all outputs to 1 or 0 . By restricting the output of the model to binary or bipolar values, one can introduce the necessary non-linearity to iteratively clean up the echo. For example, the SOB model (Farrell \& Lewandowsky; 2002) uses a matrix memory that stores items represented as vectors of $+1 \mathrm{~s}$ and $-1 \mathrm{~s}$. At retrieval, the real-valued output is upper and lower bounded by +1 and -1 , and the model continues to iterate until all values in the echo have reached +1 or -1 .

MINERVA needs to be nonlinear, as it cannot rely on a clean-up memory. MINERVA is a model of both episodic and semantic memory and thus it would "violate the spirit of MINERVA 2" (Hintzman, 1986, p. 416) to have an external store of items (i.e., a separate semantic memory) to clean up the echo.

A disadvantage of MINERVA is that it is a multi-vector memory, which raises the questions of neural plausibility and scalability. How can MINERVA be implemented in neurons if not by growing new neurons for each new experience (see Figure 5.1)? Is MINERVA practical for very long-term learning given that each experience adds another row to the memory table?

\subsection{Scalability of MINERVA}

Applying MINERVA to large scale tasks, such as learning the meaning of words (Kwantes, 2005) or learning how to sound-out written words (Kwantes \& Mewhort, 1999), requires abandoning a key assumption of MINERVA for computational efficiency, namely, that repetition of an item produces multiple traces of that item.

This might seem like an assumption that can be abandoned without affecting the model. If an item is repeated exactly, adding another row to MINERVA's table to represent the second 
occurrence of the item is mathematically equivalent to storing only one trace for that item and doubling that trace's weighting. Instead of adding additional traces, exact repetition of an item can be simulated by increasing the weight of the item's trace. While this approach suffices for modelling many experimental tasks, for real world tasks, exact repetition is an unrealistic assumption. In the words of Heraclitus, you cannot step twice into the same river.

For example, Kwantes and Mewhort's (1999) MINERVA model of sounding-out written words has one vector per word. Each vector stores a prototypical pronunciation of the word, rather than any particular experience of having heard the word pronounced, which would vary depending on context and speaker. BEAGLE (Jones \& Mewhort, 2007) and Kwantes' (2005) MINERVA model, both models of learning the meaning of words, also have one vector per word, such that all individual experiences with how a given word is used in the language are aggregated into a single vector.

For the sake of computational feasibility, as MINERVA models are scaled up, the models shift from storing individual experiences to storing individual concepts. Thus, there are two different kinds of MINERVA model: models that store individual episodes (i.e., episodic memory), and models that store generic knowledge (i.e., semantic memory). Modelling episodic and semantic memory using different assumptions violates the spirit of MINERVA 2. MINERVA 2 is intended as a model of long-term memory that accounts for both semantic and episodic memory as a single, integrated system (Hintzman, 1986). 


\subsection{Similarity in MINERVA}

Given the usefulness of using the cube of the similarity in MINERVA 2, we want to keep this feature in a variant of the model that, like matrix or tensor memories, has a memory store of fixed size. In the next section, we prove that MINERVA is mathematically equivalent to an autoassociative fourth-order tensor memory. But we must preface this proof with a discussion of how similarity is calculated in MINERVA.

MINERVA 2 (Hintzman, 1984) measures similarity between a cue and a memory trace using a normalized dot product,

$$
\frac{\mathbf{x} \cdot \mathbf{v}_{i}}{n-z}
$$

where $\cdot$ denotes the dot product, $\mathbf{x}$ is a vector representing the cue, $\mathbf{v}_{i}$ is a memory trace, $n$ is the dimensionality, and $z$ is the number of dimensions on which both $\mathbf{x}$ and $\mathbf{v}_{i}$ have a value of zero. In MINERVA 2, experiences are represented by vectors of $+1 \mathrm{~s}$ and $-1 \mathrm{~s}$. Random elements of the memory traces are set to zero to simulate memory loss. If forgetting is the only form of loss being simulated, the dot-product would be normalized by $n$, as the cue $\mathbf{x}$ contains no zeroes. However, elements of the cue may be set to zero to represent missing information, whether it is because the cue is incomplete (such as a partially occluded image) or if the cue was presented too briefly to be fully perceived by the subject. Under conditions of both an incomplete cue and forgetting, it is possible for there to be dimensions on which both the cue and the memory trace are zero (i.e., $z>0$ ).

A common technique for measuring the similarity of two vectors, used by some variants of MINERVA (e.g., Jamieson \& Mewhort, 2011) and other models of memory (e.g., Cox et al., 
2011), is to use a normalized dot product computed as the cosine of the angle between the vectors,

$$
\frac{\mathbf{x} \bullet \mathbf{v}_{i}}{\sqrt{\mathbf{x} \bullet \mathbf{x}} \sqrt{\mathbf{v}_{i} \bullet \mathbf{v}_{i}}}
$$

which normalizes the dot product by the magnitudes of the vectors. Dividing the dot product by $n-z$, as Hintzman does (1984), approximates normalizing by the magnitudes for vectors of $+1 \mathrm{~s}$, $-1 \mathrm{~s}$, and $0 \mathrm{~s}$, as used in MINERVA 2.

For the purposes of the following proof, we will assume that MINERVA uses the dot product to measure similarity. Using the dot product is equivalent to using the cosine to measure similarity if all vectors, the cue and memory traces, are normalized to a magnitude of onedoing so merely moves normalization from being part of running the model to part of generating the vectors before running the model, and so has no effect on the model's behaviour.

Conversely, using the dot product is equivalent to Hintzman's (1984) normalized dot product if $z=0$ and all vectors used in the model are normalized beforehand by scaling by $1 / n$. Thus, the following proof of equivalence is approximate rather than exact for MINERVA 2 models that use Hintzman's (1984) original measure of similarity and use both forgetting and an incomplete cue, such that $z>0$.

\subsection{MINERVA as a fourth order tensor}

In what follows, we prove equivalence between the MINERVA 2 model and an auto-associative fourth order tensor memory. To do so, we first prove that a variant of MINERVA that raises similarity to an exponent of 1 is equivalent to an auto-associative second order tensor (i.e., a matrix) memory. Then we prove that a MINERVA that uses an exponent of 2 is equivalent to a 
third order tensor. Finally, we prove that the MINERVA 2 model, which uses an exponent of 3, is equivalent to a fourth order tensor.

MINERVA with an exponent of 1 Consider a variant of MINERVA that uses dot product (denoted by $\bullet$ ) to measure similarity and weights each trace by its similarity raised to the exponent of 1. Each episode trace in memory is represented by a vector $\mathbf{v}_{i}$ where $i=1 \ldots m$ and $m$ is the number of traces in memory. When the model is presented with a cue $\mathbf{x}$, the echo $\mathbf{y}$ is:

$$
\mathbf{y}=\left(\mathbf{x} \bullet \mathbf{v}_{1}\right) \mathbf{v}_{1}+\ldots+\left(\mathbf{x} \bullet \mathbf{v}_{m}\right) \mathbf{v}_{m}
$$

This is equivalent to an auto-associative matrix memory (e.g., Farrell \& Lewandowsky, 2002).

In an auto-associative matrix memory, each episode trace is represented by a vector $\mathbf{v}_{i}$. To store a trace in memory, the trace is associated with itself (hence auto-associative) by taking the outer-product of the vector with itself, $\mathbf{v}_{i} \mathbf{v}_{i}^{T}$, then taking the sum of all the outer-product matrices to create the memory matrix, $\mathbf{M}$ :

$$
\mathbf{M}=\mathbf{v}_{1} \mathbf{v}_{1}^{\mathrm{T}}+\ldots+\mathbf{v}_{m} \mathbf{v}_{m}^{\mathrm{T}}
$$

The echo, $\mathbf{y}$, is the inner-product of the cue and the matrix:

$$
\begin{aligned}
& \mathbf{y}=\mathbf{M x} \\
& \mathbf{y}=\left(\mathbf{v}_{1} \mathbf{v}_{1}{ }^{\mathrm{T}}+\ldots+\mathbf{v}_{m} \mathbf{v}_{m}^{\mathrm{T}}\right) \mathbf{x} \\
& \mathbf{y}=\mathbf{v}_{1} \mathbf{v}_{1}{ }^{\mathrm{T}} \mathbf{x}+\ldots+\mathbf{v}_{m} \mathbf{v}_{m}^{\mathrm{T}} \mathbf{x}
\end{aligned}
$$

Because $\mathbf{v}_{i}{ }^{\mathrm{T}} \mathbf{x}$ is the dot-product of $\mathbf{v}_{i}$ and $\mathbf{x}$ :

$$
\mathbf{y}=\left(\mathbf{x} \cdot \mathbf{v}_{1}\right) \mathbf{v}_{1}+\ldots+\left(\mathbf{x} \cdot \mathbf{v}_{m}\right) \mathbf{v}_{m}
$$

which is identical to the echo from a MINERVA with an exponent of 1 . 
We note that on a recognition task, the behaviour of a MINERVA with an exponent of 1 is equivalent to the behaviour of a composite vector memory, depending on how familiarity is computed. A composite vector memory $\mathbf{m}$ is a sum of item vectors:

$$
\mathbf{m}=\mathbf{v}_{1}+\ldots+\mathbf{v}_{m}
$$

Whether or not an item $\mathbf{x}$ is recognized by $\mathbf{m}$ is a function of the familiarity, $f$, computed as the dot product of $\mathbf{x}$ with $\mathbf{m}$,

$$
f=\mathbf{x} \cdot \mathbf{m}=\left(\mathbf{x} \cdot \mathbf{v}_{1}\right)+\ldots+\left(\mathbf{x} \cdot \mathbf{v}_{m}\right)
$$

In MINERVA 2, familiarity can be computed in one of two ways. These two methods for computing familiarity are common across vector-based models of memory and are referred to as local match and global match (Kahana, Rizzuto, \& Schneider, 2005). When using a global match, familiarity is computed as the similarity between the probe and all of memory. When using local match, familiarity is computed as the similarity between the probe and the echo.

When using global match, Hintzman (1986) computes familiarity as the sum of similarities between the probe and each trace in memory. In MINERVA 2, the familiarity $f$ would be:

$$
f=\left(\mathbf{x} \cdot \mathbf{v}_{1}\right)^{3}+\ldots+\left(\mathbf{x} \cdot \mathbf{v}_{m}\right)^{3}
$$

However, when using a MINERVA with an exponent of 1 , the familiarity would equal to the familiarity computed by a composite vector model:

$$
f=\left(\mathbf{x} \cdot \mathbf{v}_{1}\right)+\ldots+\left(\mathbf{x} \cdot \mathbf{v}_{m}\right)
$$

Thus, on recognition tasks, when computing familiarity as in Hintzman (1986), a MINERVA with an exponent of 1 is equivalent to a composite vector model.

Alternatively, Hintzman (1988, p. 546) suggests that familiarity can be computed using the local match method as the similarity between the echo and the probe. Local match is used in 
some MINERVA 2 models (e.g., Jamieson and Mewhort, 2009). Because computing familiarity as the similarity between the probe and the echo allows for easier comparison to other memory models, it is the approach we adopt in our simulations (see Figures 5.2, 5.3, and 5.6).

For either MINERVA with an exponent of 1 or, equivalently, an auto-associative matrix memory, given an echo,

$$
\mathbf{y}=\left(\mathbf{x}^{\bullet} \mathbf{v}_{1}\right) \mathbf{v}_{1}+\ldots+\left(\mathbf{x} \bullet \mathbf{v}_{m}\right) \mathbf{v}_{m}
$$

we can compute the familiarity of $\mathbf{x}$ as $\mathbf{x} \cdot \mathbf{y}$,

$$
\begin{aligned}
& f=\mathbf{x} \cdot \mathbf{y}=\mathbf{x} \cdot\left(\left(\mathbf{x} \cdot \mathbf{v}_{1}\right) \mathbf{v}_{1}+\ldots+\left(\mathbf{x} \cdot \mathbf{v}_{m}\right) \mathbf{v}_{m}\right) \\
& f=\mathbf{x} \cdot\left(\mathbf{x} \cdot \mathbf{v}_{1}\right) \mathbf{v}_{1}+\ldots+\mathbf{x} \cdot\left(\mathbf{x} \cdot \mathbf{v}_{m}\right) \mathbf{v}_{m} \\
& f=\left(\mathbf{x} \cdot \mathbf{v}_{1}\right)\left(\mathbf{x} \cdot \mathbf{v}_{1}\right)+\ldots+\left(\mathbf{x} \cdot \mathbf{v}_{m}\right)\left(\mathbf{x} \cdot \mathbf{v}_{m}\right) \\
& f=\left(\mathbf{x} \cdot \mathbf{v}_{1}\right)^{2}+\ldots+\left(\mathbf{x} \cdot \mathbf{v}_{m}\right)^{2}
\end{aligned}
$$

which differs from the familiarity in a composite vector model as each term is squared. For MINERVA 2, i.e., a MINERVA with an exponent of 3, computing familiarity in this manner results in each term being raised to the power of four:

$$
f=\left(\mathbf{x} \cdot \mathbf{v}_{1}\right)^{4}+\ldots+\left(\mathbf{x} \cdot \mathbf{v}_{m}\right)^{4}
$$

In general, it seems that the difference between the local match and global match methods for computing familiarity is that if the global match method for the model in question computes familiarity as,

$$
f=\left(\mathbf{x} \bullet \mathbf{v}_{1}\right)^{n}+\ldots+\left(\mathbf{x} \bullet \mathbf{v}_{m}\right)^{n}
$$

then the local match model computes familiarity as,

$$
f=\left(\mathbf{x} \cdot \mathbf{v}_{1}\right)^{n+1}+\ldots+\left(\mathbf{x} \cdot \mathbf{v}_{m}\right)^{n+1}
$$


Thus, these two methods for computing familiarity, the global match between the probe and memory or the local match between probe and the echo, are not as different as they might seem from their initial description. However, the local match can be calculated as a cosine of the probe and the echo (i.e., a normalized dot product), as in Jamieson and Mewhort (2009). Normalizing either the global or local match may produce substantively different behavioural predictions than using the un-normalized match values, as we discuss in Section 10.5.

MINERVA with an exponent of 2 Consider a MINERVA that raises similarity to the exponent of 2:

$$
\mathbf{y}=\left(\mathbf{x} \cdot \mathbf{v}_{1}\right)^{2} \mathbf{v}_{1}+\ldots+\left(\mathbf{x} \cdot \mathbf{v}_{m}\right)^{2} \mathbf{v}_{m}
$$

This variant of MINERVA, as we shall demonstrate, is mathematically equivalent to an autoassociative third order tensor memory. Using the tensor product, denoted by $\otimes$, we can store each trace as $\mathbf{v}_{i} \otimes \mathbf{v}_{i} \otimes \mathbf{v}_{i}$, which is a third order tensor. The memory tensor $\mathbf{M}$ is the sum of the third order tensor outer-products of each episode trace:

$$
\mathbf{M}=\mathbf{v}_{1} \otimes \mathbf{v}_{1} \otimes \mathbf{v}_{1}+\ldots+\mathbf{v}_{m} \otimes \mathbf{v}_{m} \otimes \mathbf{v}_{m}
$$

The echo, $\mathbf{y}$, can be computed from the cue, $\mathbf{x}$, by taking the inner product twice:

$$
\mathbf{y}=(\mathbf{M} \mathbf{x}) \mathbf{x}
$$

If each $\mathbf{v}_{i}$ is a vector of $n$ dimensions, then $\mathbf{M}$ is an $n \times n \times n$ tensor. $\mathbf{M}$ can be thought of as $n$ matrices of $n \times \mathrm{x} n$ dimensions. When we compute the inner-product of $\mathbf{M}$ with the cue $\mathbf{x}$, we compute the inner product of $\mathbf{x}$ with each of those $n$ matrices. This results in $n$ vectors that can be rearranged into a new $n \times n$ matrix. The second inner product with $\mathbf{x}$ then produces a vector, the echo $\mathbf{y}$. 
To illustrate, let us break $\mathbf{M}$ into its components:

$$
\begin{aligned}
& \mathbf{y}=(\mathbf{M} \mathbf{x}) \mathbf{x} \\
& \mathbf{y}=\left(\left(\mathbf{v}_{1} \otimes \mathbf{v}_{1} \otimes \mathbf{v}_{1}+\ldots+\mathbf{v}_{m} \otimes \mathbf{v}_{m} \otimes \mathbf{v}_{m}\right) \mathbf{x}\right) \mathbf{x} \\
& \mathbf{y}=\left(\mathbf{v}_{1} \otimes \mathbf{v}_{1} \otimes \mathbf{v}_{1} \mathbf{x}+\ldots+\mathbf{v}_{m} \otimes \mathbf{v}_{m} \otimes \mathbf{v}_{m} \mathbf{x}\right) \mathbf{x}
\end{aligned}
$$

The tensor product $\mathbf{v}_{i} \otimes \mathbf{v}_{i} \otimes \mathbf{v}_{i}$ can be understood as $n$ matrices, where each matrix is the outerproduct $\mathbf{v}_{i} \mathbf{v}_{i}^{\mathrm{T}}$ weighted by a different element $j$ of $\mathbf{v}_{i}$, for all $j=1 \ldots n$.

$$
\mathbf{v}_{i} \otimes \mathbf{v}_{i} \otimes \mathbf{v}_{i}=\left\{v_{i 1} \mathbf{v}_{i} \mathbf{v}_{i}^{\mathrm{T}}, \ldots, v_{i n} \mathbf{v}_{i} \mathbf{v}_{i}^{\mathrm{T}}\right\}
$$

Taking the inner-product of the cue $\mathbf{x}$ with $\mathbf{v}_{i} \otimes \mathbf{v}_{i} \otimes \mathbf{v}_{i}$, we get $n$ vectors, each weighted by the dot-product of $\mathbf{x}$ with $\mathbf{v}_{i}$ :

$$
\begin{aligned}
& \mathbf{v}_{i} \otimes \mathbf{v}_{i} \otimes \mathbf{v}_{i} \mathbf{x}=\left\{v_{i 1} \mathbf{v}_{i} \mathbf{v}_{i}^{\mathrm{T}} \mathbf{x}, \ldots, v_{i n} \mathbf{v}_{i} \mathbf{v}_{i}^{\mathrm{T}} \mathbf{x}\right\} \\
& \mathbf{v}_{i} \otimes \mathbf{v}_{i} \otimes \mathbf{v}_{i} \mathbf{x}=\left\{v_{i 1}\left(\mathbf{x} \bullet \mathbf{v}_{i}\right) \mathbf{v}_{i}, \ldots, v_{i n}\left(\mathbf{x} \bullet \mathbf{v}_{i}\right) \mathbf{v}_{i}\right\}
\end{aligned}
$$

If we factor out the dot-product of $\mathbf{x}$ and $\mathbf{v}_{i}$, the result is $n$ vectors, or rather, the outer-product matrix of $\mathbf{v}_{i} \mathbf{v}_{i}^{\mathrm{T}}$ :

$$
\begin{aligned}
& \mathbf{v}_{i} \otimes \mathbf{v}_{i} \otimes \mathbf{v}_{i} \mathbf{x}=\left(\mathbf{x} \bullet \mathbf{v}_{i}\right)\left\{v_{i 1} \mathbf{v}_{i}, \ldots, v_{i n} \mathbf{v}_{i}\right\} \\
& \mathbf{v}_{i} \otimes \mathbf{v}_{i} \otimes \mathbf{v}_{i} \mathbf{x}=\left(\mathbf{x} \bullet \mathbf{v}_{i}\right) \mathbf{v}_{i} \mathbf{v}_{i}^{\mathrm{T}}
\end{aligned}
$$

Thus, when we take the outer-product of $\mathbf{x}$ with $\mathbf{M}$, the result is a sum of $m$ matrices $\mathbf{v}_{i} \mathbf{V}_{i}^{\mathrm{T}}$, each matrix weighted by the dot-product of $\mathbf{x}$ with $\mathbf{v}_{i}$.

$$
\begin{aligned}
& \mathbf{y}=\left(\mathbf{v}_{1} \otimes \mathbf{v}_{1} \otimes \mathbf{v}_{1} \mathbf{x}+\ldots+\mathbf{v}_{m} \otimes \mathbf{v}_{m} \otimes \mathbf{v}_{m} \mathbf{x}\right) \mathbf{x} \\
& \mathbf{y}=\left(\left(\mathbf{x} \bullet \mathbf{v}_{1}\right) \mathbf{v}_{1} \mathbf{v}_{1}{ }^{\mathrm{T}}+\ldots+\left(\mathbf{x} \bullet \mathbf{v}_{m}\right) \mathbf{v}_{m} \mathbf{v}_{m}{ }^{\mathrm{T}}\right) \mathbf{x}
\end{aligned}
$$

By then taking the second inner-product with $\mathbf{x}$, we reduce each matrix to a vector weighted by the squared similarity to $\mathbf{x}$, producing an echo like MINERVA with an exponent of 2:

$$
\mathbf{y}=\left(\mathbf{x} \bullet \mathbf{v}_{1}\right) \mathbf{v}_{1} \mathbf{v}_{1}{ }^{\mathrm{T}} \mathbf{x}+\ldots+\left(\mathbf{x} \bullet \mathbf{v}_{m}\right) \mathbf{v}_{m} \mathbf{v}_{m}^{\mathrm{T}} \mathbf{x}
$$




$$
\begin{aligned}
& \mathbf{y}=\left(\mathbf{x} \bullet \mathbf{v}_{1}\right)\left(\mathbf{x} \bullet \mathbf{v}_{1}\right) \mathbf{v}_{1}+\ldots+\left(\mathbf{x} \bullet \mathbf{v}_{m}\right)\left(\mathbf{x} \bullet \mathbf{v}_{m}\right) \mathbf{v}_{m} \\
& \mathbf{y}=\left(\mathbf{x} \bullet \mathbf{v}_{1}\right)^{2} \mathbf{v}_{1}+\ldots+\left(\mathbf{x} \bullet \mathbf{v}_{m}\right)^{2} \mathbf{v}_{m}
\end{aligned}
$$

Thus, an auto-associative third order tensor memory or "memory cube" is equivalent to a MINERVA with an exponent of 2. Humphreys, Bain, and Pike (1989a) use a hetero-associative third-order tensor memory that associates two different items with a context: item 1 x item $2 \mathrm{x}$ context. By contrast, the MINERVA third-order tensor memory associates an item with itself twice: item 1 x item 1 x item 1 .

MINERVA with an exponent of 3 (i.e., MINERVA 2) Given a pair of vectors that both have a magnitude of one, the dot product of those vectors is in the range of +1 to -1 . The dot product is +1 if the vectors are identical, 0 if the vectors are orthogonal, and -1 if one vector is the negation of the other. Thus it is important to preserve the sign of the dot product. By taking the square of the dot product, the sign is lost. For this reason, MINERVA 2 uses an exponent of 3. MINERVA 2 is equivalent to an auto-associative memory implemented as a fourth order tensor. Memory is constructed as a sum of fourth order tensors:

$$
\mathbf{M}=\mathbf{v}_{1} \otimes \mathbf{v}_{1} \otimes \mathbf{v}_{1} \otimes \mathbf{v}_{1}+\ldots+\mathbf{v}_{m} \otimes \mathbf{v}_{m} \otimes \mathbf{v}_{m} \otimes \mathbf{v}_{m}
$$

Given a cue $\mathbf{x}$, an echo $\mathbf{y}$ is computed by taking the inner product three times:

$$
\begin{aligned}
& \mathbf{y}=((\mathbf{M} \mathbf{x}) \mathbf{x}) \mathbf{x} \\
& \mathbf{y}=\left(\left(\left(\mathbf{v}_{1} \otimes \mathbf{v}_{1} \otimes \mathbf{v}_{1} \otimes \mathbf{v}_{1}+\ldots+\mathbf{v}_{m} \otimes \mathbf{v}_{m} \otimes \mathbf{v}_{m} \otimes \mathbf{v}_{m}\right) \mathbf{x}\right) \mathbf{x}\right) \mathbf{x} \\
& \mathbf{y}=\left(\left(\left(\mathbf{x} \bullet \mathbf{v}_{1}\right) \mathbf{v}_{1} \otimes \mathbf{v}_{1} \otimes \mathbf{v}_{1}+\ldots+\left(\mathbf{x} \bullet \mathbf{v}_{1}\right) \mathbf{v}_{m} \otimes \mathbf{v}_{m} \otimes \mathbf{v}_{m}\right) \mathbf{x}\right) \mathbf{x} \\
& \mathbf{y}=\left(\left(\left(\mathbf{x} \bullet \mathbf{v}_{1}\right)^{2} \mathbf{v}_{1} \mathbf{v}_{1}{ }^{\mathrm{T}}+\ldots+\left(\mathbf{x} \bullet \mathbf{v}_{m}\right)^{2} \mathbf{v}_{m} \mathbf{v}_{m}^{\mathrm{T}}\right) \mathbf{x}\right. \\
& \mathbf{y}=\left(\mathbf{x} \bullet \mathbf{v}_{1}\right)^{3} \mathbf{v}_{1}+\ldots+\left(\mathbf{x} \bullet \mathbf{v}_{m}\right)^{3} \mathbf{v}_{m}
\end{aligned}
$$




\subsection{Implications of the memory tesseract}

MINERVA is equivalent to a distributed memory system implemented as an auto-associative fourth order tensor, or memory tesseract. Re-interpreting MINERVA as a memory tesseract changes our understanding of the MINERVA model, and in turn, changes our understanding of what manipulations of the model are permissible.

If MINERVA is understood as a fourth-order tensor, cubing the similarity is not a parameter but a structural feature of the model. Understood as such, it is less permissible to change the value of the exponent. If we understand the GCM (Nosofsky, 1986, 1991) as a MINERVA model restricted to category judgements, then the GCM's $c$ exponent in its activation function must also be understood as a structural feature of the GCM rather than a value that can be changed at whim.

Clark and Gronlund (1996) propose a MINERVA model where information about items and the associations between them is cubed, but information about environmental context is not cubed. Implementing Clark and Gronlund's MINERVA model as a tensor would require two separate data structures: a fourth-order tensor for items and associations, and a matrix memory for contexts. Implemented in this manner, the Clark and Gronlund MINERVA would resemble Lewandowsky and Farrell (2008) C-SOB model, which also has an auto-associative memory for items and a separate matrix memory for context information. Note, though, that the C-SOB autoassociative memory for items is a matrix whereas in the Clark and Gronlund MINERVA it would be a fourth-order tensor. 
The Iterative Resonance Model (IRM; Mewhort \& Johns, 2005) is a variant of MINERVA that models retrieval from memory as an iterative process. On the first iteration, an echo is constructed as a sum of traces, each trace weighted by their similarity to the probe raised to an exponent of one. On each successive iteration, the same probe is used, but the exponent is incremented by 0.5 . By increasing the exponent with each iteration, the echo is increasingly dominated by the trace most similar to the probe. If MINERVA is understood as a tensor, increasing the exponent at each time-step during retrieval corresponds to increasing the order of the tensor, which implies changing the structure of the model during retrieval. As the exponent grows with each time-step, the tensor grows exponentially in size. Thus, the memory tesseract cannot implement the IRM as described.

We suggest that IRM could be implemented using the memory tesseract, but it would need to use a different iterative retrieval mechanism. As shown in Figure 5.2, iterative retrieval can be implemented in MINERVA with a fixed exponent of 3. On the first iteration, the probe is used to retrieve an echo. On the iterations that follow, the echo from the previous iteration is used as a new probe to retrieve a new echo. Across iterations, the echo increasingly approximates the vector in memory most similar to the original probe. This form of iterative retrieval has been used to predict reaction times in other memory models (e.g., Farrell \& Lewandowsky, 2002; Lewandowsky \& Farrell, 2008).

Just as re-interpreting MINERVA as a memory tesseract suggests that certain operations, such as changing the exponent, are impermissible, it also suggests other operations are permissible. The equivalence shows that MINERVA is not different in kind from vector, matrix, tensor, and holographic vector models. Thus, some of the techniques used in these other models 
to capture memory phenomena may be successfully applied to MINERVA. For example, MINERVA is not typically used to simulate primacy and recency effects. However, some of the other vector-based models capture one or both of these effects (e.g., Farrell \& Lewandowsky, 2002; Franklin \& Mewhort, 2015; Murdock, 1993). These models do so by applying weights to the information stored in memory.

To give a concrete example, we can adopt TODAM's (Murdock, 1993) forgetting parameter $\alpha$ and apply it to MINERVA. For each time $t$ when memory is updated with a new episode trace $\mathbf{v}_{t}$, we weight the memory tensor $\mathbf{M}$ by $\alpha$, where $0<\alpha<1$ :

$$
\mathbf{M}_{t}=\alpha \mathbf{M}_{t-1}+\mathbf{v}_{t}
$$

If the forgetting parameter $\alpha$ is less than one, newer memories are weighted more strongly during retrieval, allowing the model to simulate a recency effect. While this technique can be used regardless of whether MINERVA is implemented as a tensor or a table, the equivalence of the table to a tensor shows that these kinds of mathematical operations are permissible by MINERVA's theory.

\subsection{Is the memory tesseract practical?}

Unfortunately, fourth order tensors are very large. For most applications of MINERVA to experimental tasks, the dimensionality $n$ of a vector will be larger than the number of memories $m$ stored in the model. MINERVA, as standardly implemented, is an $m \times n$ table, whereas a memory tesseract is an $n^{4}$ data structure. A typical MINERVA 2 has $10 \leq n \leq 200$. In general, the number of memories stored is smaller than $n$ and much smaller than $n^{3}$. For applications where $m$ $<n^{3}$, the implementation of MINERVA as a table is more efficient. However, for large scale 
applications, such as modelling the lifetime learning of an agent (e.g., Jones \& Mewhort, 2007), where $m>n^{3}$, the fourth order tensor is more space efficient. However, when implemented on a serial computer, the fourth order tensor computes storage in memory $n^{3}$ times more slowly than a memory table. This is because storage in the tensor is a matter of computing a new fourth order tensor to represent the trace and adding it to the memory tensor. The storage computation for a tensor memory is prohibitively slow for large data sets processed on a serial computer.

If negative similarities have no meaning in the model, one could use a third-order tensor instead, equivalent to MINERVA with an exponent of 2. In many MINERVA models, negative similarities occur only when two vectors have approximately zero similarity, but by chance this approximate value is negative. When squaring these small, negative similarities, the sign is lost, but the sign of these approximately zero values is unimportant to the model. Such models could be reimplemented with an exponent of 2 or, equivalently, as a third-order tensor. For large-scale applications of these models, where $m>n^{2}$, the tensor implementation would be more space efficient, though storage is $n^{2}$ times slower on a serial computer. However, for some MINERVA models, negative similarities play an important role in the behaviour of the model, such as MINERVA-AL (Jamieson et al., 2012), which stores the differences between expected and observed values for the purposes of modelling learning.

Alternatively, a holographic approximation to the memory tesseract can be implemented as an $n \times p$ data structure for the $p$ of your choice, as is discussed in the next section. 


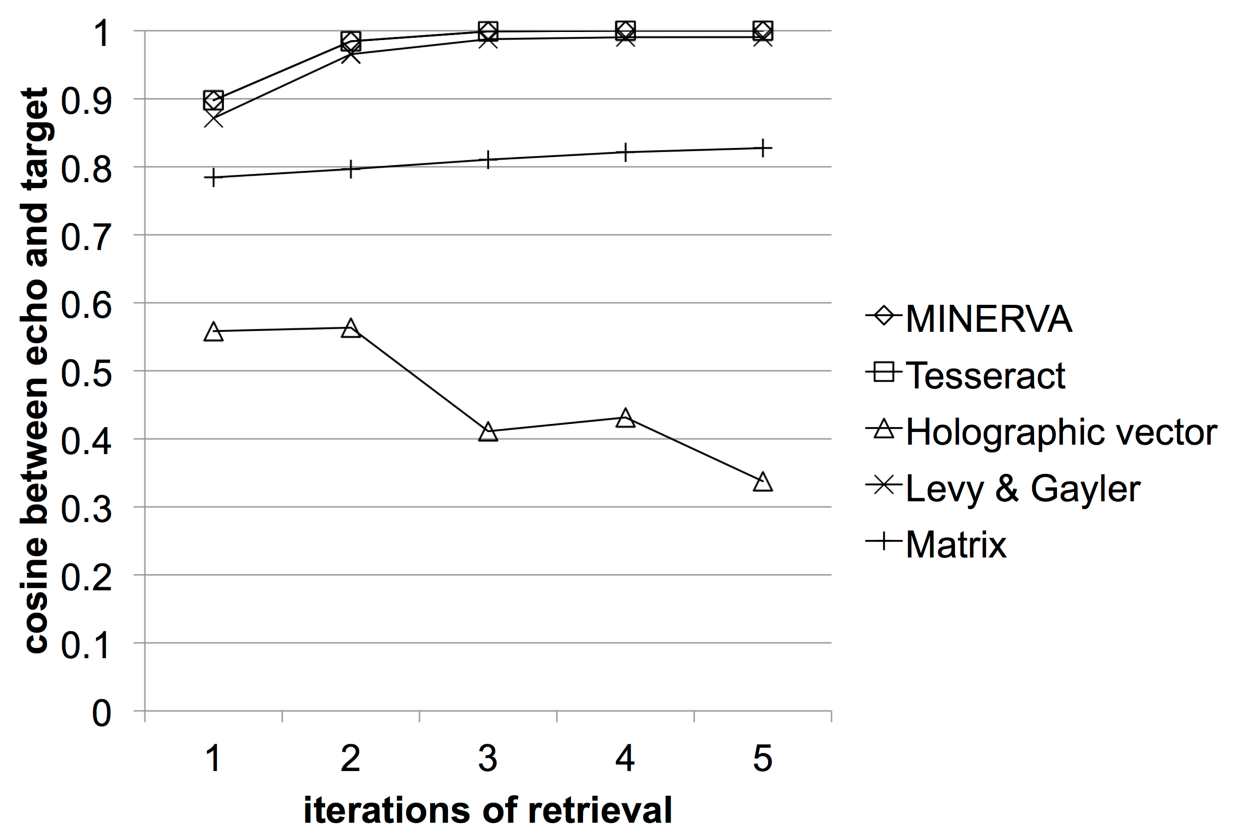

Figure 5.2. Iterative retrieval from five memory models: MINERVA 2, the memory tesseract, an auto-associative holographic vector, a variant of Levy and Gayler's (2009) model that approximates MINERVA 2 / the memory tesseract, and an auto-associative matrix memory. The 64-dimensional vectors $\mathbf{a}$ and $\mathbf{b}$ are stored in memory. On the first iteration, $\mathbf{a}+0.8 \mathbf{b}$, normalized to a magnitude of one, is used to retrieve an echo. On successive iterations, the echo from the previous iteration is used to retrieve a new echo. Cosine is measured between each echo and the target, a. Results averaged over 50 runs of each model.

\subsection{Using holographic vectors rather than tensors}

In a holographic vector memory, trying to clean-up the echo by iteratively using the echo as a cue to retrieve a new echo is like trying to clean a pair of glasses with an oily cloth: the more you try to clean it, the worse it becomes. Holographic vectors use lossy compression. With each pass through the holographic vector memory, the amount of loss, or noise, increases (see the holographic vector model in Figure 5.2).

Yet Levy and Gayler (2009) have demonstrated that it is possible to use a holographic vector system as a clean-up memory (see Levy and Gayler model in Figure 5.2). Levy and Gayler use a lateral inhibition network implemented as a fully distributed vector architecture. Gayler and 
Levy (2009) use their network to model analogical mapping, but we note that it can also be used as a memory system. Indeed, their network is approximately equivalent to a MINERVA 2 model.

To store a trace $\mathrm{v}_{i}$ in Levy and Gayler's model, the trace is associated with itself twice, then each trace is added to the memory vector $\mathbf{m}$ :

$$
\mathbf{m}=\mathbf{v}_{1} * \mathbf{v}_{1} * \mathbf{v}_{1}+\ldots+\mathbf{v}_{m} * \mathbf{v}_{m} * \mathbf{v}_{m}
$$

where * is a binding operation used to form associations. Levy and Gayler (2009) use MAP codes (Gayler, 2003), which use element-wise multiplication as a binding operation. We choose to use holographic reduced representations instead. In holographic reduced representations (Plate, 1995), binding uses circular convolution and unbinding uses circular correlation. Given a cue, $\mathbf{x}$, we can unbind, denoted by \#, to recover an echo:

$$
\begin{aligned}
& \mathbf{y}=\mathbf{x} \#(\mathbf{x} \# \mathbf{m}) \\
& \mathbf{y}=\mathbf{x} \#\left(\mathbf{x} \#\left(\mathbf{v}_{1} * \mathbf{v}_{1} * \mathbf{v}_{1}+\ldots+\mathbf{v}_{m} * \mathbf{v}_{m} * \mathbf{v}_{m}\right)\right)
\end{aligned}
$$

Unbinding is such that given a bound pair, $\mathbf{v}_{i} * \mathbf{v}_{j}$,

$$
\mathbf{x} \# \mathbf{v}_{i}^{*} \mathbf{v}_{j}=\left(\mathbf{x} \bullet \mathbf{v}_{i}\right) \mathbf{v}_{j}+\boldsymbol{\eta}
$$

where $\boldsymbol{\eta}$ is a vector of noise with a mean of zero and a normal distribution (Plate, 1995). Thus:

$$
\begin{aligned}
& \mathbf{y}=\mathbf{x} \#\left(\mathbf{x} \#\left(\mathbf{v}_{1} * \mathbf{v}_{1} * \mathbf{v}_{1}+\ldots+\mathbf{v}_{m} * \mathbf{v}_{m} * \mathbf{v}_{m}\right)\right) \\
& \mathbf{y}=\mathbf{x} \#\left(\left(\mathbf{x} \bullet \mathbf{v}_{1}\right) \mathbf{v}_{1} * \mathbf{v}_{1}+\ldots+\left(\mathbf{x} \bullet \mathbf{v}_{m}\right) \mathbf{v}_{m} * \mathbf{v}_{m}+\boldsymbol{\eta}\right) \\
& \mathbf{y}=\left(\mathbf{x} \bullet \mathbf{v}_{1}\right)^{2} \mathbf{v}_{1}+\ldots+\left(\mathbf{x} \bullet \mathbf{v}_{m}\right)^{2} \mathbf{v}_{m}+\boldsymbol{\eta}
\end{aligned}
$$

However, if we wish to imitate MINERVA 2 as closely as possible (and preserve the sign of the similarity) we need to add another association to Levy and Gayler's model. We compute the memory vector, $\mathbf{m}$, and unbind the echo, $\mathbf{y}$, as:

$$
\mathbf{m}=\mathbf{v}_{1} * \mathbf{v}_{1} * \mathbf{v}_{1} * \mathbf{v}_{1}+\ldots+\mathbf{v}_{m} * \mathbf{v}_{m} * \mathbf{v}_{m} * \mathbf{v}_{m}
$$




$$
\begin{aligned}
& \mathbf{y}=\mathbf{x} \#(\mathbf{x} \#(\mathbf{x} \# \mathbf{m})) \\
& \mathbf{y}=\mathbf{x} \#\left(\mathbf{x} \#\left(\mathbf{x} \#\left(\mathbf{v}_{1} * \mathbf{v}_{1} * \mathbf{v}_{1} * \mathbf{v}_{1}+\ldots+\mathbf{v}_{m} * \mathbf{v}_{m}{ }^{*} \mathbf{v}_{m}{ }^{*} \mathbf{v}_{m}\right)\right)\right) \\
& \mathbf{y}=\left(\mathbf{x} \bullet \mathbf{v}_{1}\right)^{3} \mathbf{v}_{1}+\ldots+\left(\mathbf{x} \bullet \mathbf{v}_{m}\right)^{3} \mathbf{v}_{m}+\boldsymbol{\eta}
\end{aligned}
$$

While this allows the most similar traces to the cue to dominate the echo, the noise term threatens to overwhelm the signal. Because holographic vectors use lossy compression, by iterating, the noise will only grow.

Levy and Gayler solve this problem by using random permutations. A random permutation is a random re-ordering of the elements in a vector, like shuffling the cards in a deck. A permutation can be represented by a permutation matrix, a square matrix with an entry of 1 exactly once in each row and column and $0 \mathrm{~s}$ in all other entries. When a vector $\mathbf{v}$ is multiplied by a permutation matrix $\mathbf{P}$, the matrix product $\mathbf{P} \mathbf{v}$ is the permuted vector.

Given three random permutation matrices $\mathbf{P}_{1}, \mathbf{P}_{2}, \mathbf{P}_{3}$, we can permute the vectors as follows:

$$
\mathbf{m}=\left(\mathbf{P}_{1} \mathbf{v}_{1}\right) *\left(\mathbf{P}_{2} \mathbf{v}_{1}\right) *\left(\mathbf{P}_{3} \mathbf{v}_{1}\right) * \mathbf{v}_{1}+\ldots+\left(\mathbf{P}_{1} \mathbf{v}_{m}\right) *\left(\mathbf{P}_{2} \mathbf{v}_{m}\right) *\left(\mathbf{P}_{3} \mathbf{v}_{m}\right) * \mathbf{v}_{m}
$$

To recover an echo, we use the permutations again:

$$
\mathbf{y}=\left(\left(\mathbf{P}_{1} \mathbf{x}\right) *\left(\mathbf{P}_{2} \mathbf{x}\right) *\left(\mathbf{P}_{3} \mathbf{x}\right)\right) \# \mathbf{m}
$$

To eliminate the noise, Levy and Gayler use hundreds of such vector memories in parallel, each of which uses its own pair, or in our variant, triple, of permutations. Let $p$ be the number of vector memories being used, then the echo $\mathbf{y}$ is the sum of echoes for each $p$ :

$$
\mathbf{y}=\mathbf{y}_{1}+\ldots+\mathbf{y}_{p}
$$

where for each $\mathbf{y}_{j}, j=1 \ldots p$,

$$
\mathbf{y}_{j}=\left(\left(\mathbf{P}_{j, 1} \mathbf{x}\right) *\left(\mathbf{P}_{j, 2} \mathbf{x}\right) *\left(\mathbf{P}_{j, 3} \mathbf{x}\right)\right) \# \mathbf{m}_{j}
$$


such that:

$$
\mathbf{y}=\left(\left(\mathbf{P}_{1,1} \mathbf{x}\right) *\left(\mathbf{P}_{1,2} \mathbf{x}\right) *\left(\mathbf{P}_{1,3} \mathbf{x}\right)\right) \# \mathbf{m}_{1}+\ldots+\left(\left(\mathbf{P}_{p, 1} \mathbf{x}\right) *\left(\mathbf{P}_{p, 2} \mathbf{x}\right) *\left(\mathbf{P}_{p, 3} \mathbf{x}\right)\right) \# \mathbf{m}_{p}
$$

Because each echo $\mathbf{y}_{j}$ is produced using a different triple of permutations, each echo's noise term will be different. Because the noise in each echo is different, a different part of the signal is preserved in each echo. By taking the sum of all these echoes, we average across them to get a close approximation to the true signal. When averaging across a set of measurements with uncorrelated error, as the number of measurements approaches infinity, the statistical error on the mean approaches zero and the mean approaches the true value. In this case, as the number of memory vectors $p$ increases to infinity, the mean echo approaches the exact value of the echo from a standard MINERVA 2. The parameter $p$ thus gives the modeller the ability to control the reliability of the model as an approximation to MINERVA 2.

With large enough $p$, we minimize the noise sufficiently that we can clean up the echo by iterating (see Figure 5.3). We divide the sum of all the echoes, $\mathbf{y}$, by its magnitude to normalize, and then use it as the new cue. By varying the number of memory vectors operating in parallel $p$, we can manipulate how quickly and how reliably the echo is cleaned up by iterating.

The number of iterations to clean up the echo is a measure of the time the model takes to perform a memory retrieval. For this reason, the number of iterations is an ideal candidate as a predictor for human reaction time. Iterations-to-clean-up-the-echo is used to predict reaction times in the SOB (Farrell \& Lewandowsky, 2002) and C-SOB (Lewandowsky \& Farrell, 2008) models of serial recall. Being able to map a measure of the model's processing time onto human reaction time helps to provide evidence for strong equivalence (Pylyshyn, 1989) between what the model is doing and what the mind is doing. 


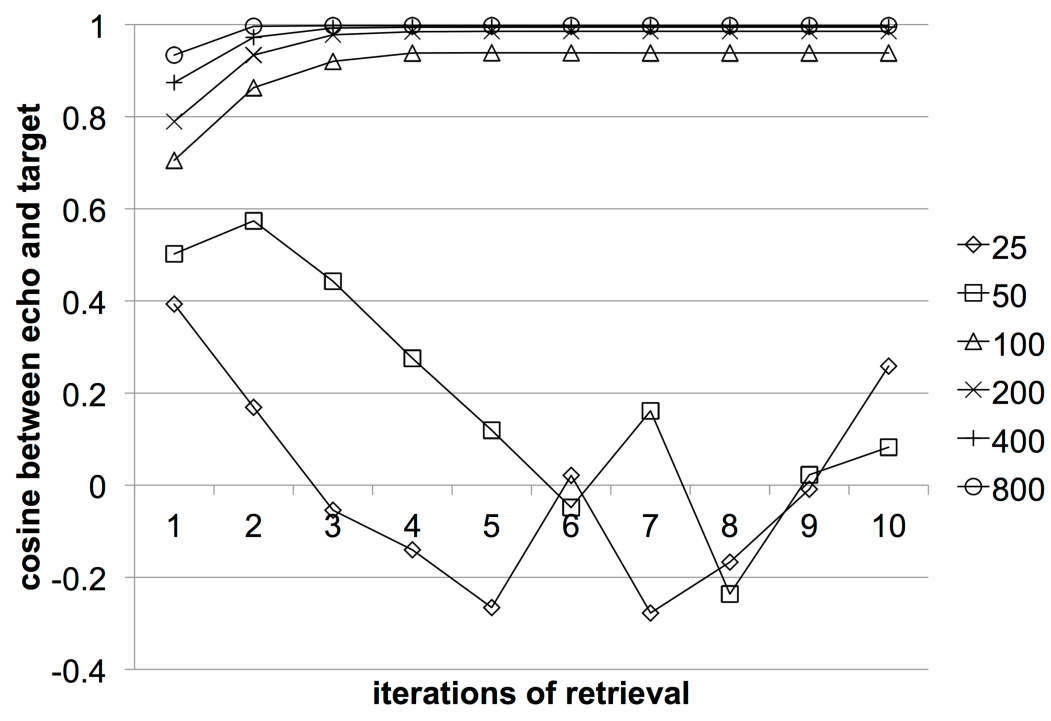

Figure 5.3. Iterative retrieval from the holographic approximation to the tesseract. As the number of memory vectors, $p$, increases from 25 to 800, the model more reliably and more rapidly cleans-up the echo. The 64-dimensional vectors $\mathbf{a}$ and $\mathbf{b}$ are stored in memory, $\mathbf{c}$ adds noise to the probe. On the first iteration, $\mathbf{a}+0.8 \mathbf{b}+\mathbf{c}$, normalized to a magnitude of one, is used to retrieve an echo. On successive iterations, the echo from the previous iteration is used to retrieve a new echo. Cosine is measured between each echo and the target, a. Results are from a single run of each model.

The number of iterations to clean up the echo in MINERVA 2 may be too few to provide sufficient granularity to map well onto human reaction times. As Hintzman (1986, p. 416) notes, for a typical experimental task, after three to four iterations, the echo will reach a steady state where it is identical to a trace in memory (see Figure 5.2). If we instead use Levy and Gayler's model, by adjusting $p$ we can vary the number of iterations that the model takes to clean up the echo (see Figure 5.3). Using smaller $p$, the model takes, typically, longer to converge to a steady state than an iterated MINERVA 2, such that the iterations map onto smaller units of human reaction time. Thus, the lateral inhibition network, when coupled with a suitable decision mechanism to end retrieval, may provide more fine-grained reaction time predictions than an iterated MINERVA 2. 
The lateral inhibition network is also more tractable than a fourth order tensor for large $n$ (i.e., more space efficient for $p<n^{3}$ and more time efficient for $p \log n<n^{3}$ ). The lateral inhibition network is also more space efficient than the table implementation for $p<m$ and more time efficient on retrieval for $p \log n<m$ but is $p \log n$ times slower at storing information in memory on a serial computer.

\subsection{Neural implementation of the holographic model}

As we have shown, MINERVA 2 is approximately equivalent to a modified version of the Levy and Gayler (2009) model. The naïve neural implementation of MINERVA commits us to the claim that a new neuron is grown for each new episode trace (Figure 5.1). However, MINERVA 2, implemented as the Levy and Gayler model, can be constructed as a biologically plausible neural model where the number of neurons is constant with respect to the number of episode traces. That such a model can be constructed serves as a proof that MINERVA 2 is not committed to the existence of the infamous grandmother cell — a neuron singly dedicated to representing a particular experience of one's grandmother.

Eliasmith’s (2013) Neural Engineering Framework ${ }^{1}$ provides a system for implementing linear algebra computations (e.g., circular convolution and permutation) in networks of biologically realistic model neurons. The Neural Engineering Framework is the basis for what is currently the largest functional brain model, the Semantic Pointer Architecture Unified Network (SPAUN; Eliasmith et al., 2012). By functional, we mean that SPAUN is the largest brain model that can perform cognitive tasks. For example, SPAUN can complete Raven's progressive

\footnotetext{
1 Models that use the Neural Engineering Framework can be constructed using the NENGO software package. For download instructions see http://www.nengo.ca/download.
} 
matrices, a simple pattern recognition task sometimes used as a test of IQ (Rasmussen \& Eliasmith, 2011). Knowledge in SPAUN is represented using holographic vectors.

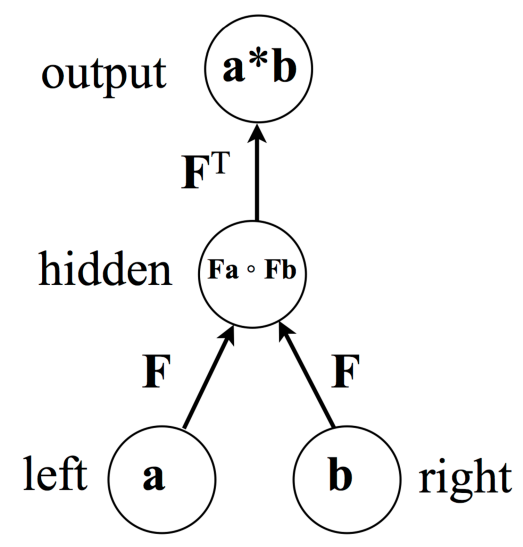

Figure 5.4. A biologically plausible neural network for computing circular convolution, implemented in the Neural Engineering Framework. Based on Figure 4.2 in Eliasmith (2013, p. 129). Left, right, hidden, and output are neural ensembles. The patterns of activation of left and right represent the vectors $\mathbf{a}$ and $\mathbf{b}$. Connections between the neural ensembles perform the Fourier transform $\mathbf{F}$ and its inverse $\mathbf{F}^{\mathrm{T}}$.

Using the Neural Engineering Framework, circular convolution can be computed in a two-layer feed-forward neural network constructed from biologically realistic model neurons (Eliasmith, 2013, p. 128; see Figure 5.4). The circular convolution * of vectors $\mathbf{a}$ and $\mathbf{b}$ can be computed as:

$$
\mathbf{a}^{*} \mathbf{b}=\mathbf{F}^{\mathrm{T}}(\mathbf{F} \mathbf{a} \circ \mathbf{F} \mathbf{b})
$$

where $\circ$ is element-wise multiplication, $\mathbf{F}$ is the matrix for the Fourier transform and $\mathbf{F}^{\mathrm{T}}$ is the transpose of that matrix, which computes the inverse Fourier transform. In other words, circular convolution is equivalent to element-wise multiplication in the frequency domain. We can compute circular convolution efficiently by using the Fourier transform to translate the vectors 
into the frequency domain, computing the element-wise multiplication, and then use the inverse Fourier transform to convert back.

Figure 5.4 is a network that computes circular convolution. The network first computes the Fourier transform of the left and right operands. The left and right operands are represented as patterns of neural activation and the connectivity from the left and right neural ensembles to the hidden layer computes the Fourier transforms of the vectors. The connectivity from the hidden layer to the output computes the element-wise multiplication of $\mathbf{F} \mathbf{a}$ and $\mathbf{F} \mathbf{b}$ and the inverse Fourier transform $\mathbf{F}^{\mathrm{T}}$, which will result in a pattern of activation at the output ensemble that corresponds to $\mathbf{a}^{*} \mathbf{b}$.

The network in Figure 5.4 can also be used to compute circular correlation. The permutation matrix $\mathbf{P}_{\text {inv }}$ is such that if $\mathbf{a}=\left\{a_{1}, a_{2}, \ldots a_{n-1}, a_{n}\right\}$, then $\mathbf{P}_{\mathbf{i n v}} \mathbf{a}=\left\{a_{1}, a_{n}, a_{n-1}, \ldots a_{3}\right.$, $\left.a_{2}\right\}$. $\mathbf{P}_{\text {inv }} \mathbf{a}$ is the approximate inverse of $\mathbf{a}$ with respect to circular convolution, such that:

$$
\mathbf{P}_{\text {inv }} \mathbf{a} *(\mathbf{a} * \mathbf{b})=\mathbf{a} \#(\mathbf{a} * \mathbf{b})=(\mathbf{a} \bullet \mathbf{a}) \mathbf{b}+\boldsymbol{\eta}
$$

where $\boldsymbol{\eta}$ is a vector of noise with a mean of zero and a normal distribution (Plate, 1995). Just as subtracting is the same as adding a negative, circular correlation is the same as convolving with the approximate inverse of a vector. Thus, if we instantiated the vectors $\mathbf{P}_{\text {inv }} \mathbf{a}$ and $\mathbf{a} * \mathbf{b}$ in the left and right ensembles of neurons in the network in Figure 5.4, the vector produced in the output ensemble would be a noisy approximation of $\mathbf{b}$. 


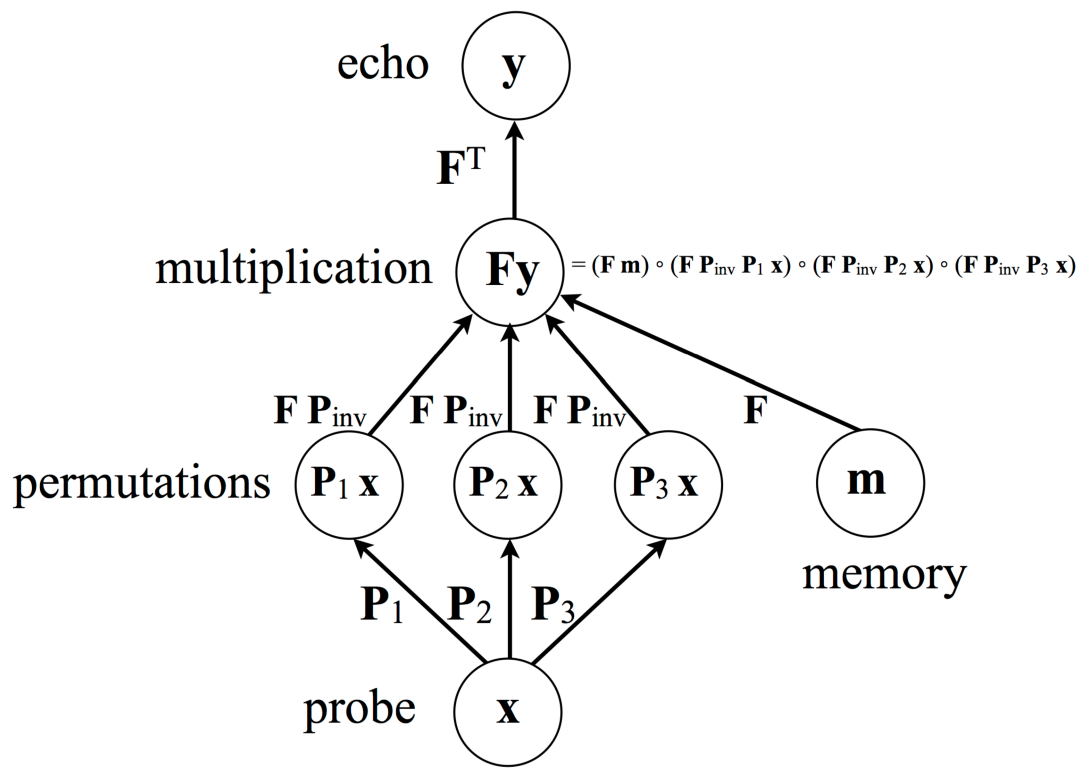

Figure 5.5. A neural model of MINERVA 2, implemented in the Neural Engineering Framework (Eliasmith, 2013). MINERVA 2 is realized as a holographic lateral inhibition network based on Levy and Gayler's (2009) model.

Using the Neural Engineering Framework, we can implement MINERVA 2, understood as the Levy and Gayler (2009) model, as a network of biologically realistic model neurons. The model is shown in Figure 5.5. The memory vector $\mathbf{m}$ is defined as in the previous section as:

$$
\mathbf{m}=\left(\mathbf{P}_{1} \mathbf{v}_{1}\right) *\left(\mathbf{P}_{2} \mathbf{v}_{1}\right) *\left(\mathbf{P}_{3} \mathbf{v}_{1}\right) * \mathbf{v}_{1}+\ldots+\left(\mathbf{P}_{1} \mathbf{v}_{m}\right) *\left(\mathbf{P}_{2} \mathbf{v}_{m}\right) *\left(\mathbf{P}_{3} \mathbf{v}_{m}\right) * \mathbf{v}_{m}
$$

Given the probe $\mathbf{x}$, the echo $\mathbf{y}$ will be a noisy approximation of the echo in a MINERVA model:

$$
\mathbf{y}=\left(\mathbf{x} \cdot \mathbf{v}_{1}\right)^{3} \mathbf{v}_{1}+\ldots+\left(\mathbf{x} \cdot \mathbf{v}_{m}\right)^{3} \mathbf{v}_{m}+\boldsymbol{\eta}
$$

In the network shown in Figure 5.5, the memory vector $\mathbf{m}$ is an input to the model in the same way that the probe $\mathbf{x}$ is an input to the model. This set up is convenient for modelling, as it allows m to be changed without needing to re-compute the neural connectivity of the network.

However, it is likely more biologically realistic for $\mathbf{m}$ to be hard-wired in the connectivity from the multiplication neural ensemble to the echo neural ensemble. 
In Figure 5.5, we see that given a probe $\mathbf{x}$ on the probe ensemble the model passes $\mathbf{x}$ forward to three ensembles of neurons. The connectivity between the probe ensemble and the three permutation ensembles multiply $\mathbf{x}$ by the permutation matrices $\mathbf{P}_{1}, \mathbf{P}_{2}$, and $\mathbf{P}_{3}$, respectively. The connections from the permutation ensembles converge on a single, large multiplication ensemble. The connections from the permutation ensembles to the multiplication ensemble multiply the vector by $\mathbf{F} \mathbf{P}_{\text {inv }}$, the product of the Fourier transform and permutation for the approximate inverse. The connections from the memory ensemble also compute the Fourier transform. The multiplication ensemble is larger than the other neural ensembles, as it needs to represent a vector of dimensionality $4 n$, where $n$ is the dimensionality of $\mathbf{x}$ and $\mathbf{m}$. The multiplication represents the vector formed from concatenating the four vectors $\mathbf{F} \mathbf{m}, \mathbf{F} \mathbf{P}_{\text {inv }} \mathbf{P}_{1} \mathbf{x}$, $\mathbf{F} \mathbf{P}_{\text {inv }} \mathbf{P}_{2} \mathbf{x}$, and $\mathbf{F} \mathbf{P}_{\text {inv }} \mathbf{P}_{3} \mathbf{x}$. As all of these vectors have been Fourier transformed, we can compute the circular convolution of these four vectors as their element-wise multiplication. The connectivity from the multiplication ensemble to the echo ensemble computes the element-wise multiplication of the four vectors to create a new $n$ dimensional vector, $\mathbf{F} \mathbf{y}$, then multiplies $\mathbf{F ~} \mathbf{y}$ by the inverse Fourier transform $\mathbf{F}^{\mathrm{T}}$ to produce the echo $\mathbf{y}$.

We do not have evidence that networks like the network in Figure 5.5 exist in the brain. However, the network serves as an existence proof that MINERVA 2 can be plausibly realized by biological neurons. Unlike the network in Figure 5.1, the network in Figure 5.5 does not commit us to growing new neurons for each new episode trace. Instead, the network can store arbitrarily many episodes in distributed representations across a fixed number of neurons.

As shown in Figure 5.3, to eliminate noise from the echo, we need to average across the echoes from hundreds of networks like the network in Figure 5.5. The networks pool their echoes 
onto a shared neural structure, which is then recurrently connected back to the networks, feeding the echo back into the individual networks to further clean up the echo. Each neural ensemble has tens of simulated neurons per dimension of the vector that the ensemble represents. As simulating hundreds of these networks at a biological level of detail requires a prohibitively large amount of computational power, all simulations described in the next section use vectors rather than neurons.

\subsection{Simulations}

To illustrate the behaviour of the models, we perform a series of simulations. In these simulations, we see that MINERVA 2 and the memory tesseract behave exactly identically, and the Levy and Gayler model (2009) closely approximates the behaviour of MINERVA 2 and the tesseract. These simulations also illustrate how these models differ from the holographic vector and matrix memory models.

All simulations for Figures 5.2, 5.3, and 5.6 were conducted using 64 dimensional vectors with values sampled randomly from a normal distribution with a mean of zero and a variance of 1/64. Each vector was then normalized to have a mean of exactly zero and a magnitude of exactly one. For the purpose of comparison, all models used the same three vectors: a, b, and c. All models had vectors a and $\mathbf{b}$ stored in memory, whereas $\mathbf{c}$ was used as a source of noise.

The MINERVA model used in all simulations is identical to Hintzman's (1984) MINERVA 2 except that it operates on vectors of normally distributed rather than binary values and correspondingly uses the cosine to measure similarity. The memory tesseract is an autoassociative fourth order tensor. Referred to as Levy and Gayler in Figures 5.2 and 5.6, the 
holographic approximation to the memory tesseract is a variant of Levy and Gayler's (2009) lateral inhibition network which, as described in the previous section, differs from Levy and Gayler's original model in that it uses holographic reduced representations (Plate, 1995) and convolves items stored in memory with themselves three times (rather than twice) in order to imitate the behaviour of MINERVA 2 and the memory tesseract. In Figures 5.2 and 5.6, the model uses $p=400$ memory vectors in parallel and averages across the 400 retrieved vectors to produce an echo. The matrix memory in Figures 5.2 and 5.6 is an auto-associative second order tensor. The holographic vector model in Figure 5.2 is a compressed version of the matrix memory that uses convolution rather than the outer product to form associations.

Figure 5.2 illustrates iterative retrieval from five memory models: MINERVA 2, the memory tesseract (behaviour is identical to MINERVA 2), the holographic approximation to the memory tesseract, and a matrix memory. On the first iteration, the probe $\mathbf{a}+0.8 \mathbf{b}$, normalized to a magnitude of one, is used to retrieve an echo. On successive iterations, the echo from the previous iteration is used to retrieve a new echo. Cosine is measured between each echo and the target, a. Results are averaged over 50 runs of each model. For each run of the five models, a different $\mathbf{a}, \mathbf{b}$, and $\mathbf{c}$ are randomly generated. All models use the same $50 \mathbf{a}, \mathbf{b}$, and $\mathbf{c}$.

In Figure 5.2, we can see that MINERVA 2, the memory tesseract, and the Levy and Gayler model all rapidly clean up the probe $\mathbf{a}+0.8 \mathbf{b}$, nearly perfectly reproducing $\mathbf{a}$ by iteration 3 or 4. The matrix memory grows to resemble a slightly more across successive iterations. Conversely, the echo of the holographic vector memory degrades across iterations. Figure 5.2 illustrates that MINERVA 2 and equivalent (or approximately equivalent) models, do not require an external clean-up memory, whereas a holographic vector memory does. 
Figure 5.3 illustrates iterative retrieval from the holographic approximation to the tesseract with a varying number of memory vectors $p$. As the number of memory vectors, $p$, increases from 25 to 800, the model more reliably and more rapidly cleans-up the echo. On the first iteration, the probe $\mathbf{a}+0.8 \mathbf{b}+\mathbf{c}$, normalized to a magnitude of one, is used to retrieve an echo. On successive iterations, the echo from the previous iteration is used to retrieve a new echo. Cosine is measured between each echo and the target, a. Results are from a single run of each model, with all models run on the same set of vectors $\mathbf{a}, \mathbf{b}$, and $\mathbf{c}$. We chose not to average across runs because the average behaviour of the model is not representative of the actual behaviour of the model, particularly for small values of $p$ (see $p=25$ and $p=50$ in Figure 5.3).

In Figure 5.6, we present the results of a simulation of four memory models alongside each other: MINERVA, the memory tesseract, the holographic approximation to the memory tesseract, and a matrix memory. In Figure 5.6, all models were presented with four probes: $\mathbf{a}+\mathbf{c}, \mathbf{a}$ $+\mathbf{b}, \mathbf{a}+2 \mathbf{b}$, and $-\mathbf{a}$, with results shown in Figure 5.6 (i), (ii), (iii), and (iv) respectively. Given a probe, each model retrieved an echo. Figure 5.6 shows the cosine similarity that each echo has with the vectors $\mathbf{a}, \mathbf{b}, \mathbf{c}$, and the probe that elicited the echo. Results are averaged across 10 different randomly generated vectors $\mathbf{a}, \mathbf{b}$, and $\mathbf{c}$.

In Figure 5.6, we see that the behaviour of MINERVA and the memory tesseract model is exactly identical, as expected given the previously presented proof of mathematical equivalence. The behaviour of all four models is approximately the same for probes (i), (ii), and (iv). In Figure 5.6 (i), given the probe $\mathbf{a}+\mathbf{c}$, the models return an echo that is nearly identical to a and does not contain the unstudied item c. In Figure 5.6 (ii), given a probe $\mathbf{a}+\mathbf{b}$, that is an equal mix of two studied items, all models return an echo identical, or nearly identical, to $\mathbf{a}+\mathbf{b}$. The echoes are 
caught between the two attractors, $\mathbf{a}$ and $\mathbf{b}$. In Figure 5.6 (iv), we see that given the probe $-\mathbf{a}$ all models produce an echo identical to, or nearly identical to, -a. This demonstrates that all of the models preserve the sign of the cosine, as we would expect given that all of the models use an odd-numbered exponent (an exponent of 1 for the matrix memory and an exponent of 3 for the other models).

(i) Probe a+c

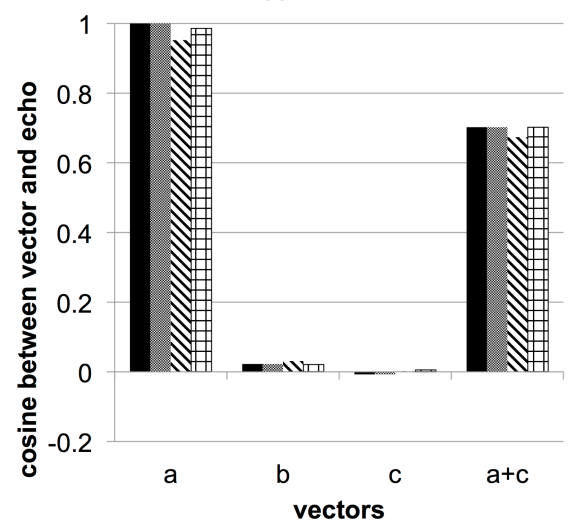

(iii) Probe $\mathbf{a}+2 \mathrm{~b}$

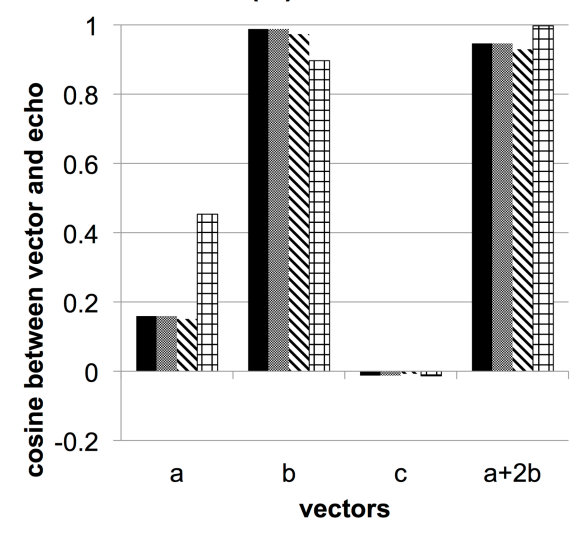

(ii) Probe $a+b$

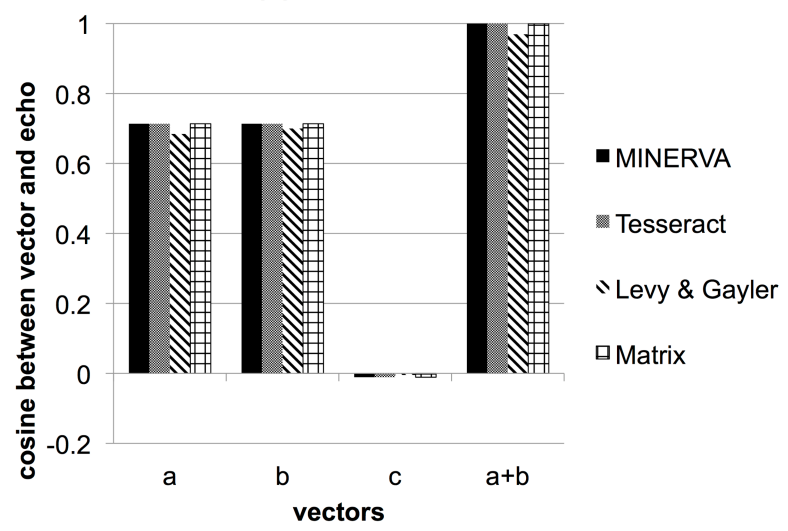

(iv) Probe -a

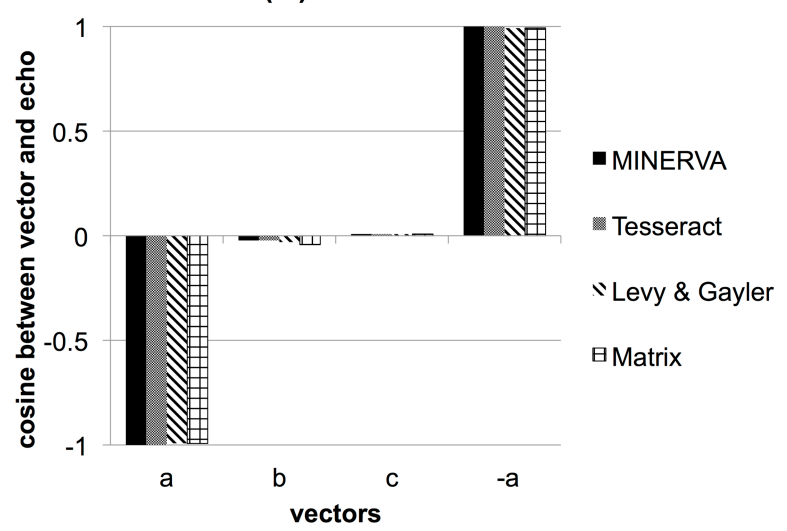

Figure 5.6. A comparison of four memory models: MINERVA, the memory tesseract, the holographic approximation to the memory tesseract, and an auto-associative matrix memory. Four different vectors (probes) are presented to all models: (i) $\mathbf{a}+\mathbf{c}$, (ii) $\mathbf{a}+\mathbf{b}$, (iii) $\mathbf{a}+2 \mathbf{b}$, (iv) $\mathbf{- a}$. Four corresponding vectors (echoes) are retrieved from each model. Plotted is the cosine between each echo and the vectors $\mathbf{a}, \mathbf{b}, \mathbf{c}$, and the probe that elicited the echo. 
Figure 5.6 (iii) demonstrates the difference in behaviour between the (linear) matrix memory and the other (non-linear) models. Given the probe $\mathbf{a}+2 \mathbf{b}$, the matrix memory produces an echo nearly identical to the probe $(\operatorname{cosine}=1.00)$. Conversely, MINERVA 2 and the memory tesseract produce an echo which is nearly identical to $\mathbf{b}(\operatorname{cosine}=0.99)$ and only weakly similar to $\mathbf{a}(\operatorname{cosine}=0.16)$. The behaviour of the holographic model closely approximates MINERVA 2 and the memory tesseract, but with added noise.

\subsection{Approximating the Exemplar Production Model}

To provide a larger-scale demonstration of the memory tesseract, we replicate a simulation result from Johns et al. (2016). Johns et al. demonstrate that a MINERVA model trained on exemplar sentences can infer the syntactic structure of novel sentences. Johns et al. name their model the Exemplar Production Model (EPM).

EPM represents each sentence as a pair of vectors. One vector represents the sentences as an unordered set of words. The other vector represents the words as an ordered sequence. EPM stores pairs of these $n$ dimensional vectors as concatenated vectors of $2 n$ dimensions. Given a probe that represents an unordered set of words, EPM can retrieve an echo, the latter half of which indicates the order of the words according to the model's experience. EPM is trained on a study set of 1000 to 125000 sentences and then tested on 200 novel sentences. Johns et al. use sentences of three to seven words.

Johns et al. represent each word as a vector of 1024 dimensions. An unordered representation of a sentence is a sum of the vectors that represent the words in the sentence. An ordered representation of a sentence is a holographic vector, a sum of convolutions and 
permutations of vectors representing words and positions in the sentence. Each trace stored in the model is a 2048 dimensional concatenation of an ordered and unordered representation. In the largest case, when EPM is trained on 125000 sentences, EPM's memory is a $125000 \times 2048$ dimensional matrix.

Using the holographic approximation to the memory tesseract, we replicate Johns et al.'s simulation of syntactic inference from 125000 seven word sentences. To create the study set, we sampled 125000 seven word sentences from a corpus of novels selected from Project Gutenberg$^{2}$. The test sets from Johns et al. can be downloaded from Johns' website ${ }^{3}$. Due to differences in how contractions are represented in the corpus versus the test set (e.g., is haven't two words or one?), we removed 40 sentences with contractions from the test set. We used the remaining 160 seven word sentences as the test set.

For seven word sentences and a study set of 125000 sentences, Johns et al. report that EPM finds the correct ordering of words for $52 \%$ of the sentences in the test set. Notably, EPM uses an exponent of 9 instead of 3. Running EPM on our 125000 word study set and the 160 sentence test set, we get 65\% correct using 1024 dimensional vectors for words (2048 for traces) and an exponent of 9 (the same parameters used by Johns et al.).

We find that after 10 iterations to clean up the echo, performance decreases to $53 \%$ correct, which suggests that with this data set and parameters, iterating is introducing noise into the echo rather than cleaning up. We also find that if we decrease the exponent to 3, EPM gets only $16 \%$ correct, and after 10 iterations, only $5 \%$ correct. This suggests that MINERVA 2's canonical exponent of 3 is insufficient when large quantities of data are stored in memory.

\footnotetext{
${ }^{2}$ http://www.gutenberg.org

${ }^{3}$ http://btjohns.com/experience_sents.zip
} 


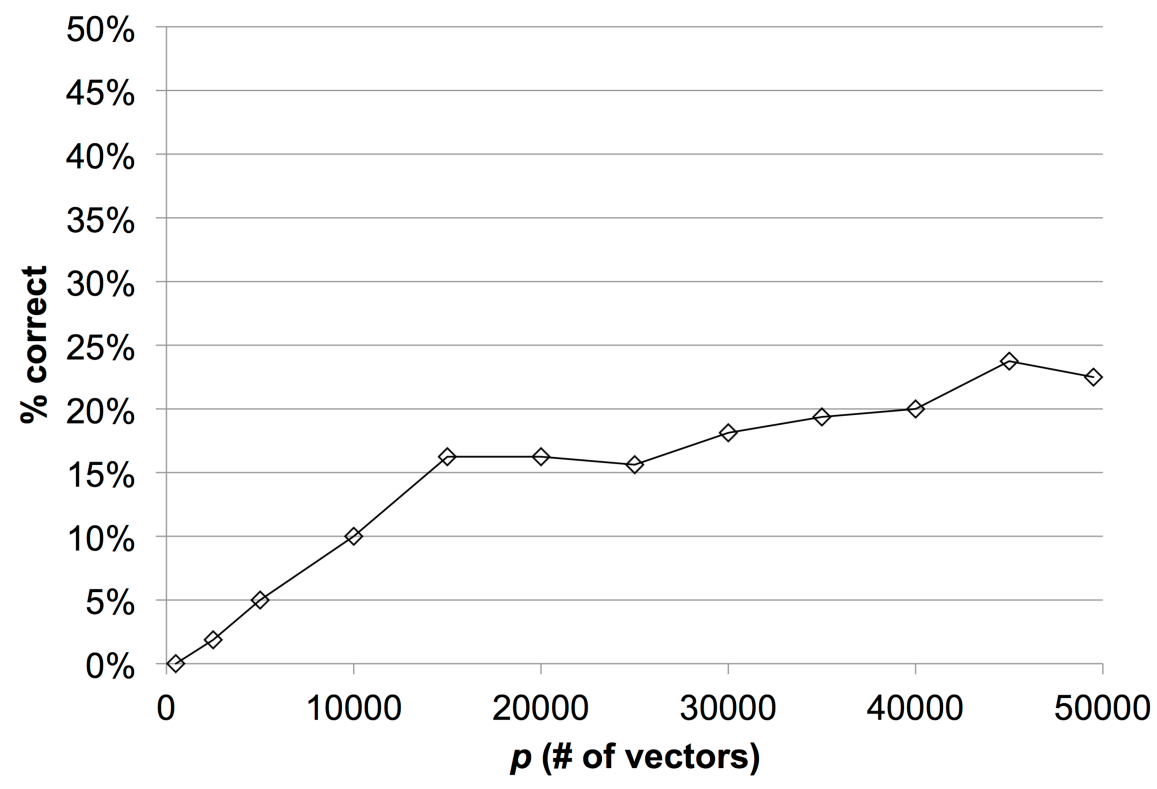

Figure 5.7. Percentage of test sentences ordered correctly by the holographic approximation to EPM as a function of the number of memory vectors, $p$.

EPM is equivalent to a tenth order tensor, a data structure of $2048^{10}$ values. A holographic vector implementation approximates that tensor, compressing $2048^{10}$ values into a vector of 2048 values. We choose to use a variant of EPM with an exponent of 5 instead of 9 to reduce the amount of compression. With an exponent of 5, the holographic vectors compress $2048^{5}$ into 2048. With an exponent of 5, we find that EPM gets $42 \%$ correct. We simulate the holographic approximation to EPM with up to $p=50000$ vectors and get, at best, $24 \%$ correct on the test items (see Figure 5.7).

The model is judged to have selected the correct ordering when the vector representing the correct ordering is more similar to the echo than the vectors representing any other possible ordering. For a seven-word sentence, there are $7 !=5040$ different orderings of the words in the sentence. These 5040 orderings are ranked from most similar to the echo to least. If the correct 
ordering is assigned a rank of one, then the model is judged to be correct on that test item. The mean and median ranks provide a more sensitive measure of the model's performance. With a power of 5, EPM's echoes have a mean rank of 35.4 and a median rank of 2. For the holographic approximation to EPM, at 50000 vectors, the mean rank is 224.5 and the median rank is 9 . Performance of the holographic model seems to approach an asymptote as the number of memory vectors $p$ increases, such that there is diminishing improvement in performance as more vectors are added (see Figure 5.8).

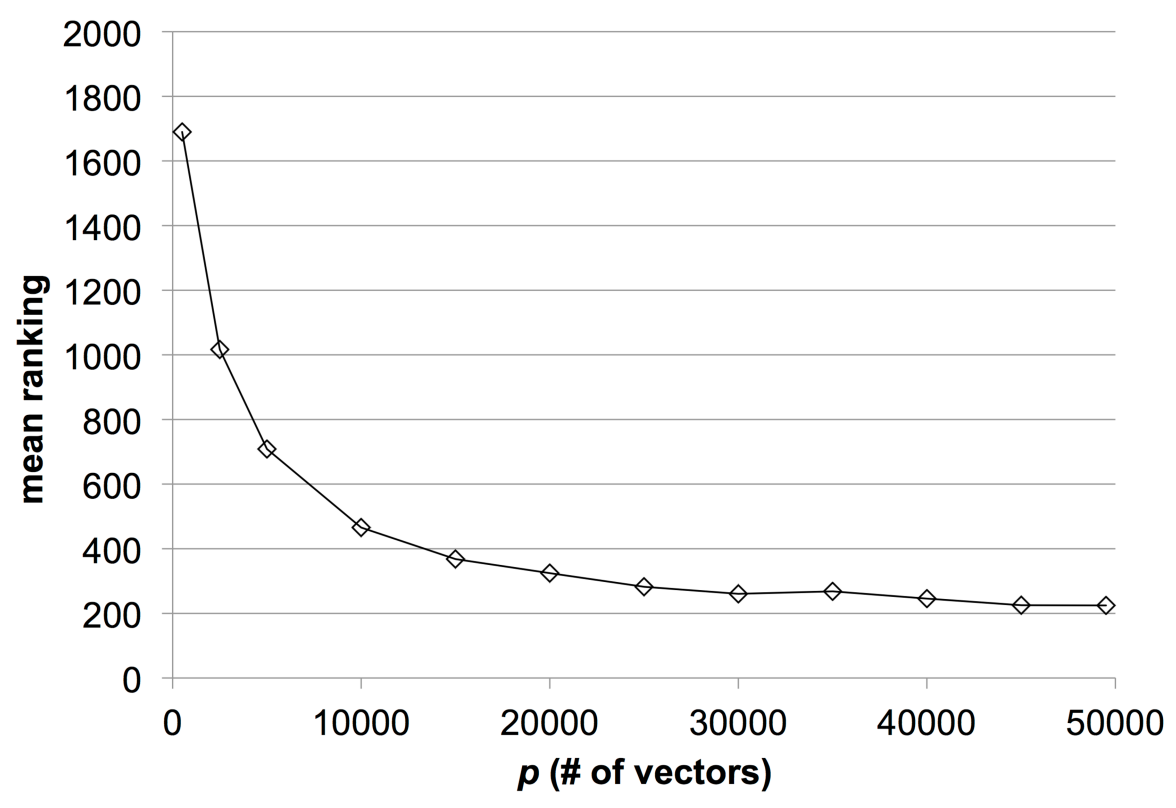

Figure 5.8. Mean ranking of the correct ordering of words in test sentences. Performance of the holographic approximation to EPM as a function of the number of memory vectors, $p$.

This simulation demonstrates that larger scale MINERVA models can be re-implemented as the holographic approximation. However, representing memory traces as distributed across a set of memory vectors introduces additional noise, particularly when compressing and storing the memory traces across a set of memory vectors fewer than the number of memory traces. For 
applications where fine discrimination is critical (such as discriminating between 5040 highly similar alternatives), this added noise might not be inconsequential to performance of the model.

Additionally, the holographic approximation is impractical to implement on a serial computer. On a serial computer, storing a memory trace across 50000 memory vectors takes 50 000 times longer than storing a memory trace in MINERVA. MINERVA's implementation as a table is preferable assuming a serial computer with unlimited storage.

This simulation does, however, demonstrate that MINERVA can be re-implemented as a massively parallel memory system with a fixed amount of storage. As the brain is also a massively parallel computer with limited storage, this demonstration lends support to MINERVA's validity as a theory of the memory algorithms implemented in the brain.

The holographic approximation may also be a practical implementation of MINERVA for large-scale modelling applications that use neuromorphic computers. Neuromorphic computers include the SpiNNaker architecture (Furber, Galluppi, Temple, \& Plana, 2014), which has been used for simulating neural models of learning and memory (Knight, Voelker, Mundy, Eliasmith $\&$ Furber, in press).

\subsection{Discussion}

According to Popper's (1959) falsificationism, science progresses by seeking evidence against theories and then rejecting those theories once that evidence is found. Lakatos (1976) refines the Popperian model of science, noting that while individual models and hypotheses may be refuted, scientists operate within research programs that have a core set of assumptions that are rarely revised. Computational theories of memory can be understood as Lakatosian programs, with core 
sets of assumptions that are not usually changed by the empirical refutation of an instantiation of the model. As Clark and Gronlund (1996, p. 40) note:

First, each of the [memory] models should be viewed as a framework within which specific models can be proposed. These frameworks evolve under the pressure of empirical data, and, although specific versions of models may be falsified by data, it is rare that the entire framework will be falsified.

Each computational memory model is a framework that evolves to meet the demands of capturing new empirical phenomena. The mathematical and computational assumptions held in common across these models can be understood as a higher-level framework that captures what researchers believe to be true of human memory.

Hintzman's MINERVA 2 model of memory is demonstrably false. MINERVA 2 has been falsified repeatedly by various experimental phenomena. But the MINERVA theoretical framework is not falsified by these demonstrations. Rather, the MINERVA framework evolves.

For example, Mewhort and Johns (2005) show that MINERVA 2 cannot account for response times in the correct rejection of a foil. To address this failing of the model, Mewhort and Johns propose the Iterative Resonance Model, a variant of MINERVA that incorporates a more detailed account of the temporal course of retrieval and a more elaborate decision mechanism for rejecting foils. Likewise, Jamieson et al. (2012) augment MINERVA with an error-correction learning mechanism to model associative learning phenomena. Thanks to the development of variants on Hintzman's MINERVA 2, the MINERVA theoretical framework continues to make scientific progress. 
The MINERVA theoretical framework is defined by a set of key assumptions. We address two questions about these key assumptions that affect the validity of the framework as a whole: (1) Hintzman's (1990) network model of MINERVA requires a new node to be added to the network for each new experience (see Figure 5.1). Is it possible for the MINERVA architecture to be realized in the brain without making the unrealistic assumption that a new neuron needs to be grown for each new experience?

(2) Is it computationally feasible to scale MINERVA to large-scale tasks without abandoning the assumption of one memory trace per experience?

To address these questions, we examine the mathematical structure of MINERVA and demonstrate that MINERVA is exactly equivalent to a composite auto-associative memory implemented as a fourth order tensor and approximately equivalent to a holographic vector memory that represents a compressed fourth order tensor. We term this fourth order tensor memory system a 'memory tesseract'.

We show that, compressed into a holographic vector, the memory tesseract can be realized by a neural network constructed from biologically realistic simulated neurons. MINERVA, as described by Hintzman (1986), is a massively parallel architecture, but with localist storage that grows linearly with the number of stored experiences. Understood as a memory tesseract compressed into a holographic vector, MINERVA is a massively parallel architecture, but with distributed storage that is fixed in scale with respect to the number of stored experiences. While prohibitive to simulate on a serial computer, the holographic approximation to MINERVA demonstrates that MINERVA can be scaled to arbitrarily large-scale tasks if we assume a massively parallel realization, as Hintzman (1986) is committed to, and as 
would be plausibly found in the brain.

This work contributes to developing a unified mathematical and computational basis for modelling memory. At the heart of memory theory is the Hebbian learning rule: neurons that fire together wire together. If the activation patterns of two groups of connected neurons can be represented by a pair of vectors, then the outer-product of those vectors describes the connections that will form between the neurons, represented as a matrix of connection weights. Abstracting away from the neural details, Smolensky (1990) notes that, in general, an association between $k$ items can be represented as a tensor of order $k$. We see this observation reflected in the memory models in the literature. Composite vector memories are tensors of order one that store single items. Matrix memories are tensors of order two that store pairs of representations. '3D' matrix memories are tensors of order three that store triples of representations. Collectively, we can refer to these memory models as tensor memories.

Holographic vectors (Plate, 1995) and the related vector-symbolic architectures (see Kelly et al., 2013 for review) are tensors compressed into vectors. A holographic vector of fixed dimensionality can encode associations between $k$ representations for arbitrary $k$. Holographic vectors thus provide a computationally efficient basis for modelling memory for arbitrarily complex associations. Collectively, we can refer to vector-symbolic / holographic vector models and tensor models as composite memory models. All share the same Hebbian learning underpinning, although abstracted away from the details of biological neurons.

In the literature, multi-vector (or separate storage) memory models have been regarded as distinct from composite memory models (e.g., Clark and Gronlund, 1996). By proving that MINERVA is equivalent to a fourth order tensor memory, we show that these two classes of 
models are not different in kind. Additionally, we show that large-scale 'semantic' memory models such as BEAGLE and smaller-scale 'episodic' memory models such as MINERVA are also not different in kind. Thus, the models discussed in this chapter can be understood as part of a single mathematical framework. We would expect that, for the most part, advances made for any particular one of these memory models could be translated into an advance for any other of these memory models. Thus, the profusion of memory models in the literature may not pose as much difficulty for developing a unified model of human memory as it might initially seem.

An important question in the development of a unified model of human memory will be settling the issue of representation. We have noted that different (tensor) models of memory adopt different structures (different orders of tensor) to model different tasks. Holographic vectors provide a way of building a memory model with an efficiently computable structure that is invariant with respect to changes in the task demands. But this does not solve the problem that the representation scheme used changes from task to task. Memory models do not explain how these representations arise, which allows the modeller to assume whatever representations are most convenient for modelling the given experimental task.

To some extent the ad hoc approach to modelling representation is justifiable, as humans encode information in different tasks differently so as to best meet the demands of the task (Hintzman, 2016). But to advance closer to a complete theoretical understanding of human memory, memory models need to be integrated with a theory of representation, and hence, perception. Some work has been done towards this goal — the SPAUN brain model (Eliasmith et al., 2012) incorporates both perception and memory_-but much more work needs to be done in integrating perception and memory if we are to achieve a full understanding of either. 


\subsection{Conclusion}

We demonstrate that the influential MINERVA 2 (Hintzman, 1984) model is mathematically equivalent to an auto-associative fourth order tensor memory system, or memory tesseract. We further show that this is approximately equivalent to a variant of the holographic lateral inhibition network proposed by Levy and Gayler (2009). These demonstrations have three theoretical implications:

(1) Viewing MINERVA 2 and its variants (collectively, MINERVA models) as a fourth order tensor clarifies the relationship between MINERVA and third order, second order (i.e., matrix), first order (i.e., composite vector), and compressed tensor (i.e., holographic vector) memory models, allowing us to move toward a unified basis for models of memory.

(2) Because MINERVA can be implemented as a fully distributed memory system that is invariant in scale with respect to the number of experiences stored in the model, MINERVA can, in principle, be scaled up to model arbitrarily long-term learning. However, simulating MINERVA as a tensor or holographic vector is most feasible on a massively parallel computer.

(3) A naïve neural interpretation of MINERVA might suggest that a new neuron is grown for each new experience, corresponding to the addition of another row to MINERVA's memory table. Understood as a memory tesseract, MINERVA is fully distributed across neural connectivity, and memories can be added by changing the connectivity without requiring additional neural resources. Eliasmith's (2013) Neural Engineering Framework (NEF) provides a system for translating linear algebra computations (e.g., convolution, permutation) into spiking neuron models. MINERVA can be implemented as a neural model using the NEF. 


\section{Chapter 6 Proof of Concept Neural Implementation of MINERVA}

I demonstrate that MINERVA 2 (Hintzman, 1984) can be accurately approximated by the behaviour of realistic spiking neurons using the Neural Engineering Framework (NEF; Eliasmith, 2013) and Neural Engineering Objects (NENGO) software. MINERVA 2 is a model of long-term memory that can account for a wide variety of behavioural phenomena and continues to be used by memory researchers today. Implementing MINERVA 2 in spiking neurons provides evidence that the MINERVA 2 model is neurally plausible. Additionally, this work suggests that MINERVA 2 would be a suitable candidate for modelling long-term memory in SPAUN, the world's largest functional brain model. I demonstrate that MINERVA 2 can be implemented in NENGO as both a recurrent and non-recurrent model, requiring about 7000 neurons for 16 dimensional vectors. To use this model to make experimental predictions will require integration with a control system, such as SPAUN's basal ganglia model.

\subsection{The Semantic Pointer Unified Architecture}

The Semantic Pointer Architecture Unified Network (SPAUN; Eliasmith, 2013) is the world's largest functional brain model. While not the largest brain model, it is the largest model that performs cognitive tasks. For example, SPAUN can complete Raven's progressive matrices, a simple pattern recognition task sometimes used as a test of IQ (Rasmussen \& Eliasmith, 2011).

SPAUN is built using the Neural Engineering Framework (NEF; Eliasmith, 2013). NEF is a theory of how functions are implemented in the brain. NEF has been implemented by Eliasmith's lab as the Neural Engineering Objects (NENGO) software for creating 
neurocognitive models. SPAUN integrates several NENGO models of specific tasks and cognitive functions into a single model of the brain.

SPAUN is a computational cognitive architecture: a system that models human cognition by presenting a formal description of the algorithms that underlie all human cognition. Ideally, a cognitive architecture describes all of the algorithms believed to be necessary to model human cognition, but in practice, all cognitive architectures are incomplete. SPAUN is a relatively new cognitive architecture, and so there is much that still needs to be added to SPAUN. SPAUN is a model of a few select brain areas and is far from being a model of the entire brain.

Though SPAUN lacks a model of long-term memory, SPAUN is not without memory systems. SPAUN has active memory, that is, data held in neural buffers. A neural buffer consists of a recurrent neural loop that allows a pattern of activation to persist across time. SPAUN's active memory is used to store small quantities of data, such as a list of digits, for later retrieval. SPAUN's basal ganglia (Stewart, Bekolay, \& Eliasmith, 2012) also serves as a sort of memory system. The basal ganglia model is like a production system. The neural connectivity in the basal ganglia encodes a set of condition-action pairs. When a pattern of activation is passed into the basal ganglia model, the condition that most closely matches the pattern of activation is selected, and the action associated with that condition is executed by the basal ganglia model. The basal ganglia model is an example of what I term here a passive memory: data is stored in the structure of the neural connectivity rather than in the activity of the neurons.

The basal ganglia model is not intended as a generic memory store. For one, the basal ganglia model is a localist memory store: each production is instantiated by a distinct neural structure. This model would suggest that there are relatively small number of productions, at 
least in comparison to the number of individual memories that make up a human lifetime of experiences. Secondly, the basal ganglia model acts too rapidly to account for human reaction times in memory recall tasks, though the basal ganglia model's speed is appropriate for modelling executive control.

SPAUN does not yet have a passive memory store for episodic or semantic memories. A MINERVA memory model is a possible candidate.

\subsection{Implementing MINERVA in NENGO}

I have implemented the basic functionality of MINERVA using the Neural Engineering Objects (NENGO) software.

The straightforward way to implement MINERVA in NENGO is to implement retrieval from memory in MINERVA as a function in the Python programming language, then create a set of neural connections in NENGO that are generated to approximate the MINERVA retrieval function. In NENGO, this is as simple as setting function=minerva when coding the neural connection.

\subsection{Testing the MINERVA Model}

There are limitations to the ability of NENGO's neurons to approximate a given arbitrary function. For functions that are higher-order polynomials, more neurons are required. For instance, to achieve the same amount of error, a first order polynomial function requires about 64 neurons, whereas a fourth order polynomial function requires about 1024 neurons (Eliasmith, n.d.). Because MINERVA cubes the cosines between the cue and each episode trace, MINERVA 
requires a large number of neurons. Through trial and error, I have found that a small MINERVA memory model that uses 16-dimensional vectors to represent memory episodes requires around 7000 neurons to work reliably. In Kelly, Mewhort, and West (2014) I demonstrate that MINERVA can be understood as a distributed, associative memory. When understood as such, MINERVA is a fourth order tensor, a data structure of $n^{4}$ dimensions, where $n$ is the dimensionality of the vectors stored. Given that $16^{4}$ is 65536 , it is perhaps less surprising that NENGO requires as many as 7000 neurons to model a 16-dimensional MINERVA.

To test the model, I first consider a 4-dimensional MINERVA with 4000 neurons that stores three orthogonal vectors: $\mathbf{a}=[1,0,0,0], \mathbf{b}=[0,1,0,0]$, and $\mathbf{c}=[0,0,1,0]$. A fourth vector, $\mathbf{d}=[0,0,0,1]$ serves as a control. Typically, MINERVA uses random vectors, but for this test I use orthogonal vectors instead to eliminate random similarities between vectors as a source of noise. I cue the model with the vector $\mathbf{q}=\mathbf{c}+0.5 \mathbf{b}=[0,0.5,1,0]$.

I test three variants of MINERVA: MINERVA with an exponent of one (i.e., a linear variant of MINERVA), MINERVA with an exponent of two, and MINERVA with an exponent of three (i.e., standard MINERVA). For each variant of MINERVA, I compare two NENGO models: a neural model that approximates MINERVA, and a model that computes MINERVA directly instead of using neurons (by using direct mode in NENGO). By comparing these versions of the model, I show that the NENGO neural model reproduces the effect of increasing the exponent.

\subsection{Results}

For the linear MINERVA model, the echo, e, is identical to the cue, $\mathbf{q}$, i.e., $\operatorname{cosine}(\mathbf{e}, \mathbf{q})=1$.

$$
\mathbf{e}=\operatorname{cosine}(\mathbf{q}, \mathbf{a}) \mathbf{a}+\operatorname{cosine}(\mathbf{q}, \mathbf{b}) \mathbf{b}+\operatorname{cosine}(\mathbf{q}, \mathbf{c}) \mathbf{c}
$$




$$
\begin{aligned}
& \mathbf{e}=[0,0.4472,0.8944,0] \\
& \mathbf{e}=0.8944 \mathbf{q}
\end{aligned}
$$

As shown in Figure 6.1, the neural model can reproduce the behaviour of MINERVA with an exponent of one. For MINERVA with an exponent of two, the echo is calculated as:

$$
\begin{aligned}
& \mathbf{e}=\operatorname{cosine}(\mathbf{q}, \mathbf{a})^{2} \mathbf{a}+\operatorname{cosine}(\mathbf{q}, \mathbf{b})^{2} \mathbf{b}+\operatorname{cosine}(\mathbf{q}, \mathbf{c})^{2} \mathbf{c} \\
& \mathbf{e}=[0,0.2000,0.8000,0]
\end{aligned}
$$

The higher exponent emphasizes the contribution of $\mathbf{c}$ over $\mathbf{b}$ in the echo. Figure 6.2 shows that the neural model again reproduces the desired behaviour. Finally, in standard MINERVA, the echo is computed with an exponent of three:

$$
\begin{aligned}
& \mathbf{e}=\operatorname{cosine}(\mathbf{q}, \mathbf{a})^{2} \mathbf{a}+\operatorname{cosine}(\mathbf{q}, \mathbf{b})^{2} \mathbf{b}+\operatorname{cosine}(\mathbf{q}, \mathbf{c})^{2} \mathbf{c} \\
& \mathbf{e}=[0,0.0894,0.7155,0]
\end{aligned}
$$

Figure 6.3 shows that the neural model successfully approximates the behaviour of the standard MINERVA model.
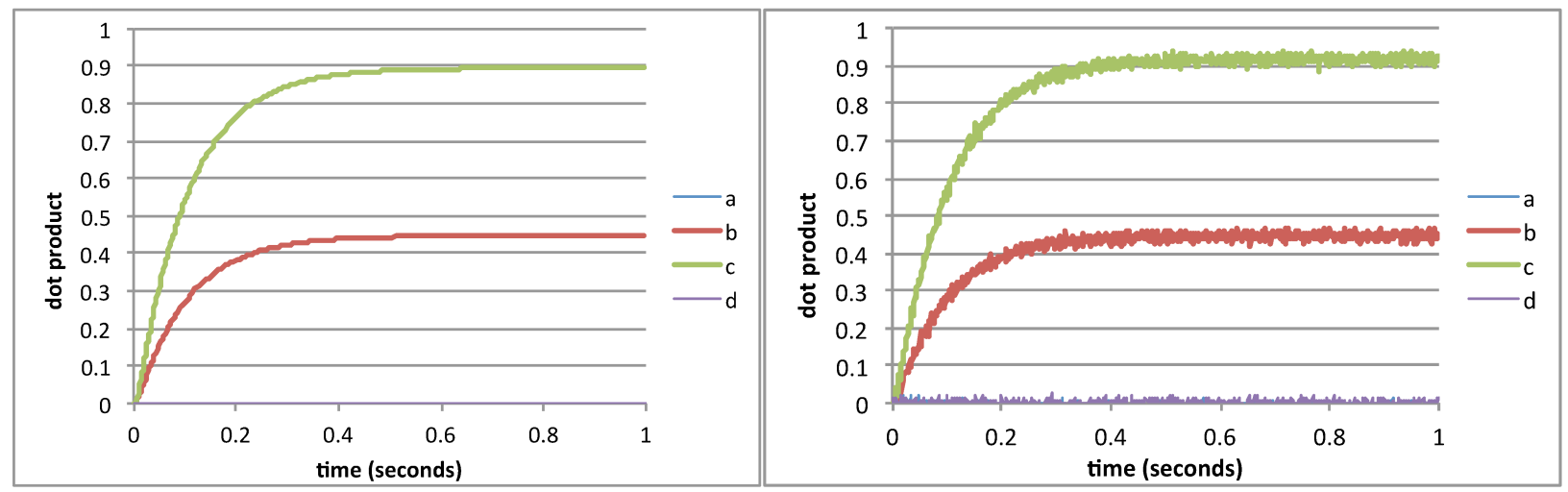

Figure 6.1. Echo from MINERVA with an exponent of 1. Direct (left) and neural model (right). 


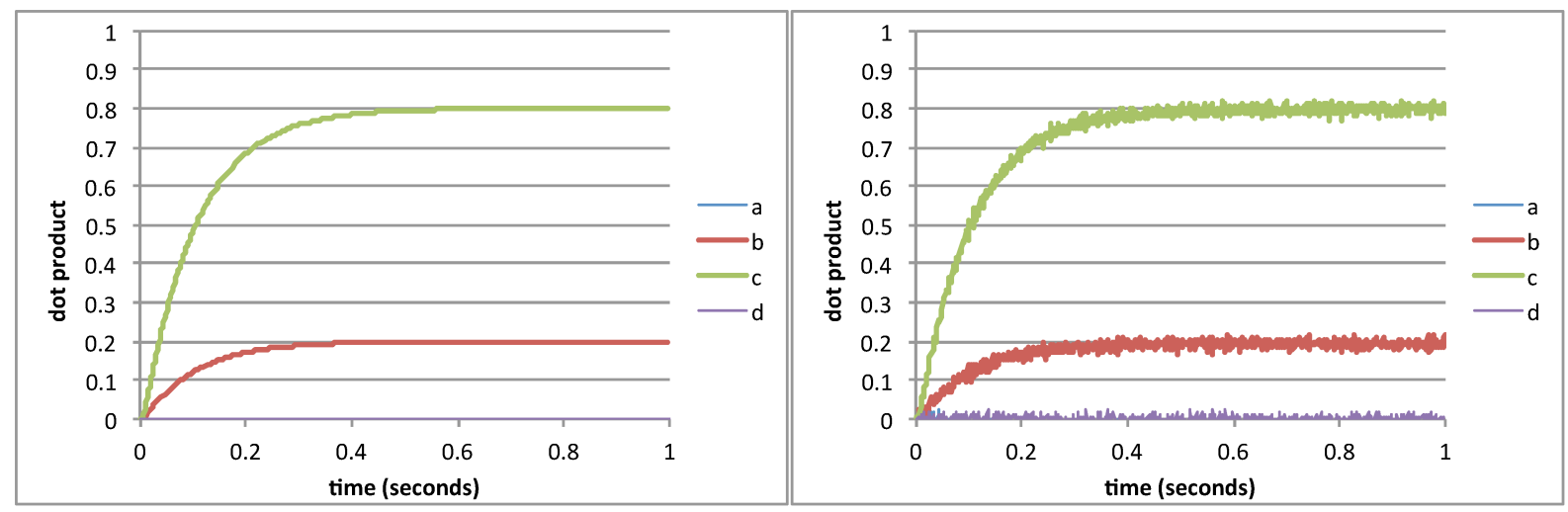

Figure 6.2. Echo from MINERVA with an exponent of 2. Direct (left) and neural model (right).

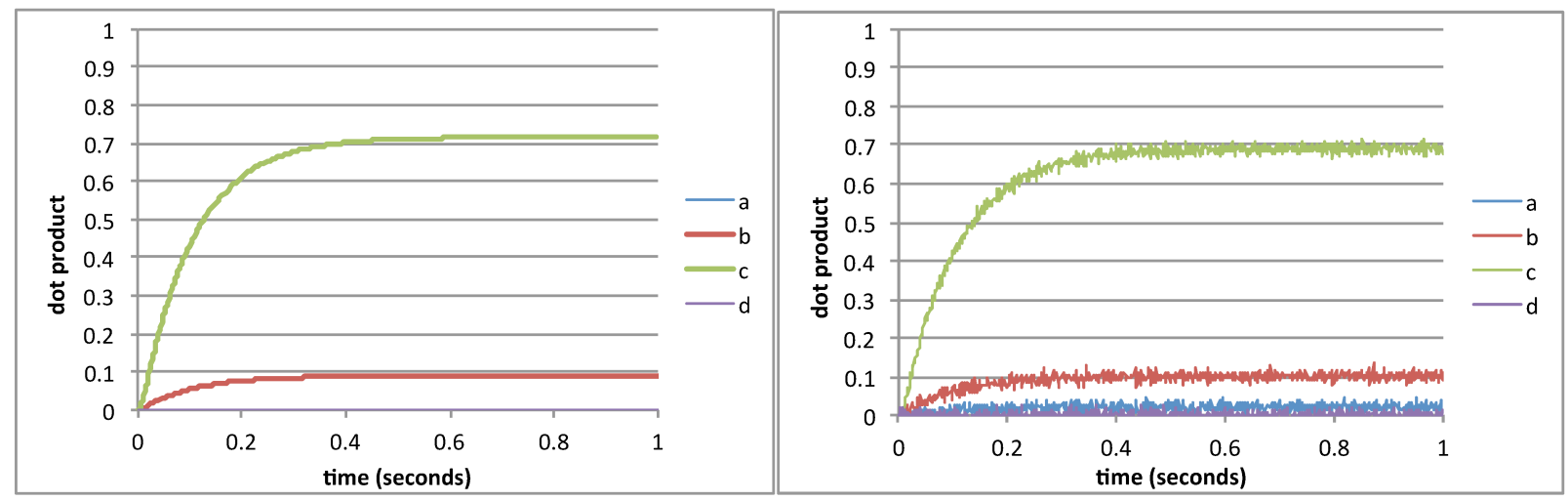

Figure 6.3. Echo from MINERVA with an exponent of 3. Direct (left) and neural model (right).

\subsection{Recurrent MINERVA in NENGO}

In NENGO, the echo can can be passed through MINERVA repeatedly by creating a recurrent set of connections from the output ensemble to the input ensemble. I set the connections from the output ensemble to the input ensemble to also compute MINERVA, such that a signal passing through the input ensemble to the output ensemble and back again passes through MINERVA twice.

The recurrent network is illustrated in Figure 6.4. The cue is passed from the stimulus node to the direct (top part of the network) and neural (bottom of the part of the network) versions of the model. Each model consists of two ensembles. The recurrent connections 
between the input and output ensembles compute the MINERVA model. The pattern of activation at the both of the output ensembles are continuously written to a file.

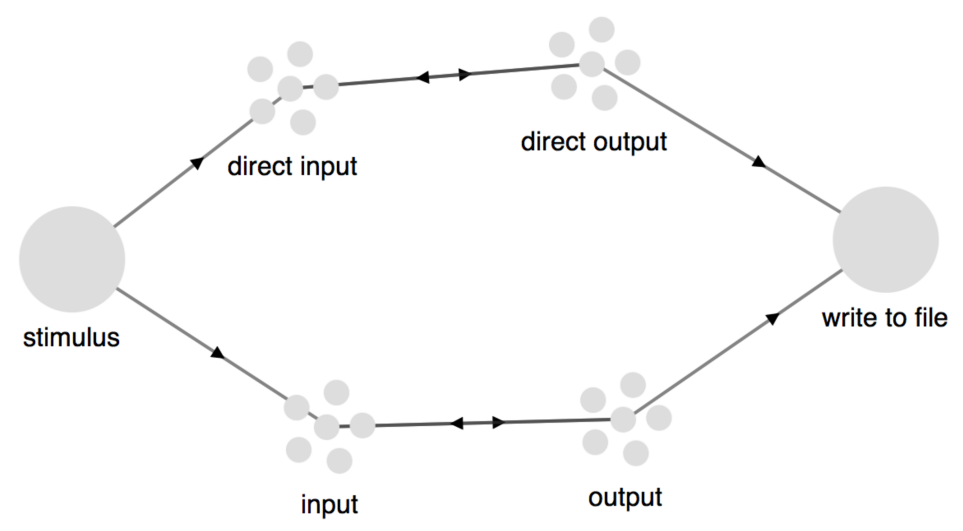

Figure 6.4. The direct (top) and neural (bottom) recurrent MINERVA models in NENGO.

To test the recurrent version of the model, I use random vectors of 16 dimensions and neural ensembles with 7000 neurons each. As before, MINERVA stores three vectors, a, $\mathbf{b}$, and $\mathbf{c}$, but these vectors are random and only approximately orthogonal (cosines between vectors are set to be less than 0.05). The cue is, as before, $0.5 \mathbf{b}+1.0 \mathbf{c}$. The cue is presented to the model for 0.3 seconds and then removed.

As shown in Figure 6.5, the NENGO implementation of MINERVA is an effective cleanup memory. The vector $\mathbf{c}$, the strongest signal in the cue, rapidly dominates, and the competing signal $\mathbf{b}$ is diminished. Conversely, as shown in Figure 6.6, a recurrent, linear variant of MINERVA (i.e., with an exponent of 1) is pointless, as passing the cue through a linear variant of MINERVA merely reproduces the cue's 2:1 ratio of $\mathbf{c}$ to $\mathbf{b}$, no clean-up occurs. 


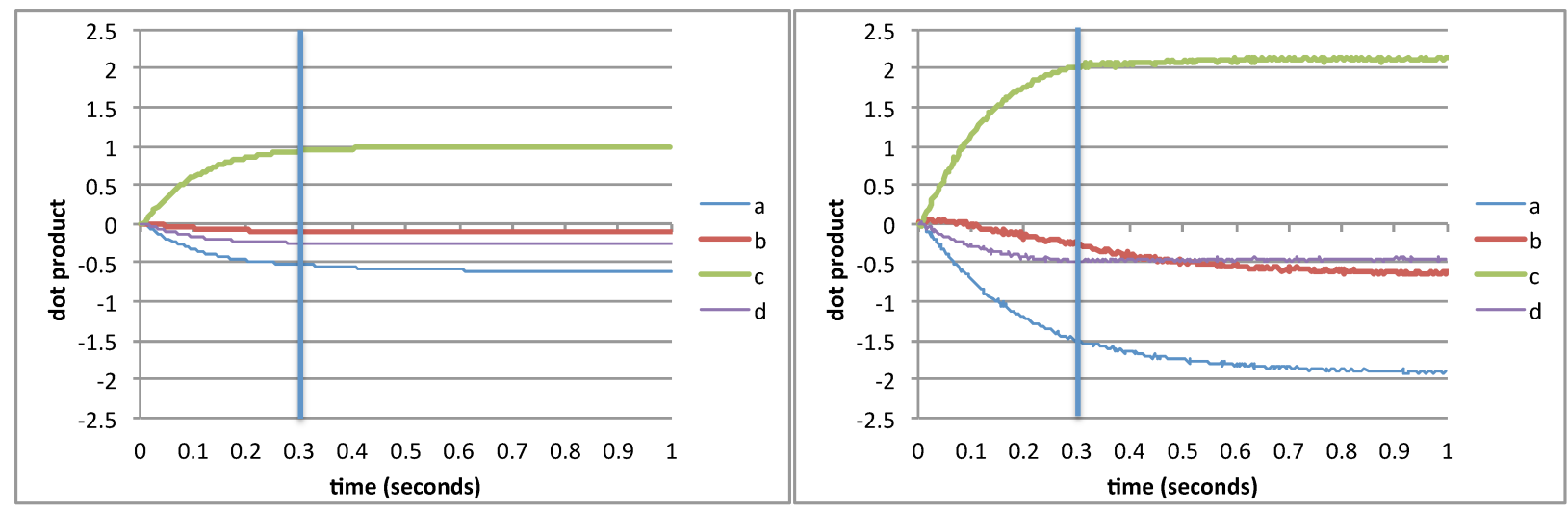

Figure 6.5. Echo from the recurrent MINERVA model with an exponent of 3 (standard model). Direct model (left) and neural model (right). Vertical Bar at 0.3 seconds indicates removal of the stimulus.

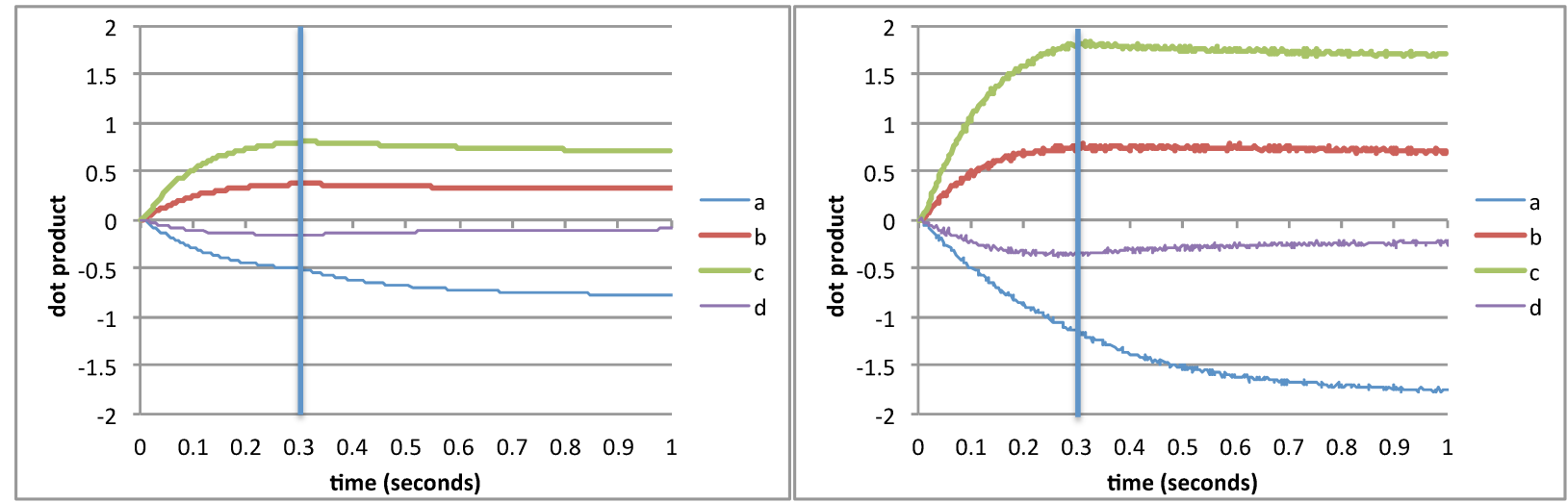

Figure 6.6. Echo from the recurrent MINERVA model with an exponent of 1 (linear model). Direct model (left) and neural model (right). Bar at 0.3 seconds indicates removal of the stimulus.

\subsection{Discussion}

The preceding results demonstrate that:

(1) Hintzman's (1984) MINERVA 2 memory model can be accurately approximated by a realistic, spiking neuron model constructed using the NENGO platform.

(2) The MINERVA 2 memory model can be implemented as a recurrent neural network in NENGO, allowing the model to act as a "clean up" memory. That is to say, the similarity 
of the echo to the most similar item in memory can be improved by recurrently passing the echo through the network.

To use this model to perform a memory task it will be necessary to connect the model to a control system that passes the model stimuli and uses the echoes from the model to respond. The control system will need a way to clear the memory buffer to perform a new retrieval. A simple way to do this might be to have the neural MINERVA model strongly favour external input over the signals from its own recurrent connections. If that approach is used, it will be necessary to have the control system turn off external input to allow the model to perform recurrent clean-up of the echo unimpeded.

I believe it is possible to use this model, when connected to a suitable control system, to generate accuracy and reaction time data for memory tasks, but it may not be straightforward to do so. MINERVA typically uses the cosine between the cue and the echo to make accuracy and reaction time predictions, but the model is vague about how this is done. The Iterative Resonance Model (IRM; Mewhort \& Johns, 2005) hypothesizes that resonance is an iterated process that progressively retrieves from memory a clearer and more accurate representation. The process continues to iterate until a decision is made. The faster the echo reaches clarity the faster a decision can be made. While the iterative-resonance model proposes a time-varying process (namely, resonance) to account for reaction time differences, the precise mechanism proposed by the model is implausible. To increase the clarity, with each iteration IRM increments the exponent. In a neural model, incrementing the exponent is impossible as it would require changing the neural connectivity and increasing the number of neurons with each time step of the 
retrieval process. A neural, recurrent MINERVA may provide part of the time-varying process necessary for making reaction time predictions, but this has not been tested.

One matter of concern is the fact the NENGO model uses 7000 neurons for a conservative 16 dimensions. To reduce the necessary neural resources, one could use an exponent of 2 rather than 3. By using an exponent of 2, however, the sign of the similarity is lost. As long as all negative similarities in the task domain are near zero, a MINERVA model with an exponent of 2 should be a workable alternative. There is also another way to create a neural implementation of MINERVA that may be more efficient for high dimensional vectors.

\subsection{Holographic Approximation to MINERVA}

As I discuss in the previous chapter, an approximation to MINERVA can be implemented using holographic vectors. The downside is that the holographic vector approximation to MINERVA is a noisy approximation. In order for the model to work at all, it's necessary to average the outputs across hundreds of holographic approximations to MINERVA to cancel out the noise.

Retrieval in MINERVA is equivalent to computing the inner-product of a fourth-order tensor. Understood as a tensor, MINERVA is an $n^{4}$ data structure. Conversely, the holographic approximation to MINERVA is a $p n$ data structure for a large $p$ (e.g., $p=500$ for $n=512$ ), such that the memory system is more reliable larger $p$. For $n^{3}>p$, the holographic approximation is more computationally efficient than the tensor implementation. If the NENGO model behaves like the tensor implementation of MINERVA in that the number of neurons required increases exponentially with the number of dimensions, it may be preferable to use a NENGO implementation of the holographic approximation to the MINERVA model. 


\subsection{Conclusions}

Hintzman's (1984) MINERVA 2 memory model can be accurately approximated by a spiking neuron model implemented in the Neural Engineering Framework (NEF) using the Neural Engineering Objects (NENGO) software. I demonstrate performance of a NENGO implementation of MINERVA 2's retrieval function. I find that both non-recurrent and recurrent versions of the model work correctly when allocated enough neurons. For 16 dimensional vectors, I found by trial and error that around 7000 neurons were necessary. Reducing the exponent of MINERVA to 2 or using the holographic approximation to MINERVA may provide means of implementing MINERVA in NENGO using fewer neurons.

This work presents evidence that MINERVA 2 is a neurally plausible model of human memory and suggests MINERVA 2 as a candidate for modelling long-term memory in a neurocognitive brain model such as SPAUN.

The MINERVA 2 model implemented in NENGO has not been tested against human behaviour in any of the paradigms that MINERVA 2 has an established ability to model in the literature. Using the NENGO model of MINERVA to make predictions on experimental memory tasks would require integrating the memory model with an appropriate control system, such as SPAUN's basal ganglia model. 


\section{Chapter 7 The Fan Effect and ACT-R}

We present Holographic Declarative Memory (HDM), a new memory module for ACT-R and alternative to ACT-R's Declarative Memory (DM). ACT-R is a widely used cognitive architecture that models many different aspects of cognition, but is limited by its use of symbols to represent concepts or stimuli. HDM replaces the symbols with holographic vectors.

Holographic vectors retain the expressive power of symbols but have a similarity metric, allowing for shades of meaning, fault tolerance, and lossy compression. The purpose of HDM is to enhance ACT-R's ability to learn associations, learn over the long-term, and store large quantities of data. To demonstrate HDM, we fit performance of an ACT-R model that uses HDM to a benchmark memory task, the fan effect. We analyze how HDM produces the fan effect and how HDM relates to the standard DM model of the fan effect. We find that the vector algebra of HDM estimates conditional probabilities in a manner similar to quantum models of human probability judgement.

\subsection{Introduction}

Computational cognitive architectures provide the formal, unified theories of cognition necessary for cognitive scientists to achieve an understanding of the mind. ACT-R (Anderson \& Lebiere, 1998) is a widely used cognitive architecture that can model diverse aspects of cognition. As an integrated architecture, ACT-R is a good choice for modelling complex tasks. However, the ACTR Declarative Memory system (DM) was designed for modelling the results of psychology experiments and as such presents certain limitations for modelling complex, real world 
behaviour. In what follows, we present Holographic Declarative Memory (HDM), a new module for the ACT-R cognitive architecture that addresses some of DM's limitations. To help establish that HDM can provide the same functionality as ACT-R's DM, we have modelled the fan effect task (Anderson, 1974), analyzed how HDM generates the fan effect, and used this analysis to compare the HDM and DM models.

Holographic Declarative Memory (HDM) replaces ACT-R's symbols with holographic vectors. Holographic vectors retain the expressive power of symbols but have a similarity metric, allowing for shades of meaning, fault tolerance, and lossy compression of stored information.

HDM is based on BEAGLE (Jones \& Mewhort, 2007), a learning algorithm that models how people abstract the meaning of words from their lifetime language experience, and DSHM (Rutledge-Taylor, Kelly, West, \& Pyke, 2014), a model that uses a similar approach to BEAGLE but re-purposes and extends the algorithm as a general memory model. HDM is implemented for Python ACT-R and the code for both Python ACT-R and HDM are available through GitHub ${ }^{4}$. Our intent with HDM is to replicate the basic functionality of DM and provide new capabilities.

First, we provide an introduction to holographic models of memory and the fan effect. Next, we detail Anderson and Reder's (1999) ACT-R model of the fan effect. We then describe HDM and the ACT-R HDM model of the fan effect. We contribute a novel analysis of how holographic models produce the fan effect and relate to Anderson and Reder's model. Finally, we outline future work.

\footnotetext{
${ }^{4}$ A Python ACT-R distribution with HDM included can be downloaded from $<$ https://github.com/MatthewAKelly/ ccmsuite $>$ and the fan effect model, which requires Python ACT-R and HDM, can be downloaded from <https:// github.com/MatthewAKelly/faneffect>. A guide to using Python ACT-R can be found at $<$ https://sites.google.com/ site/pythonactr/>.
} 


\subsection{Holographic Models of Memory}

First proposed by Longuet-Higgins (1968) and Gabor (1969), a holographic memory is a type of computational associative memory based on the mathematics of holography. Holographic memory has been of interest to cognitive psychologists because of the following:

(1) Associative memories are content-addressable, allowing for retrieval without search.

(2) Holographic memories compactly store complicated, recursive relations between ideas.

(3) Holographic memories have "lossy" storage, useful for modelling human forgetting. Cognitive models based on holographic memory can explain and predict a variety of human memory phenomena, such as the serial position curve in free recall (Franklin \& Mewhort, 2015). Holographic memory has also been used to model analogical reasoning (Plate, 2000; Eliasmith \& Thagard, 2001) and how humans perform simple problem-solving tasks such as playing rocks, paper, scissors (DSHM; Rutledge-Taylor et al., 2014) or solving Raven's progressive matrices (Eliasmith, 2013). Knowledge in SPAUN, the world's largest functional brain model (Eliasmith, 2013), is represented using holographic memory.

ACT-R DM is not designed for modelling tasks that involve large databases, such as language comprehension. Conversely, BEAGLE (Jones \& Mewhort, 2007) and DSHM (Rutledge-Taylor, Vellino, \& West, 2008) are holographic models that have been used, respectively, to infer word meanings from a corpus and to infer patterns of movie preferences from a database of user movie scores. 
Holographic memory models have also been previously used to model the fan effect. Specifically, Dynamically Structured Holographic Memory (DSHM; Rutledge-Taylor et al., 2014; Rutledge-Taylor, Pyke, West, \& Lang, 2010) has been used to model two versions of the fan effect task.

Though HDM is based on DSHM, the HDM module for ACT-R differs sufficiently from DSHM that it is worth demonstrating that HDM can, in fact, model the fan effect task. The differences between HDM and DSHM stem from HDM's integration into ACT-R. As a module for ACT-R, HDM makes commitments as to the cognitive structure that the memory system is situated in. To interface with ACT-R, HDM commits to a particular way of encoding information and to a particular way of calculating reaction times that are distinct from the DSHM model.

\subsection{Fan Effect}

The fan effect task (Anderson, 1974) is a recognition memory task. During the study phase of the task, participants memorize a set of sentences that vary on some number of dimensions. In the original fan effect task (Anderson, 1974), each sentence is of the form "the person is in the location" where the person and location vary (e.g., "the hippie is in the park").

Once the participants have the sentences memorized, they are given a recognition task. In the recognition task, some sentences are from the study set (targets), and some sentences are novel combinations of the people and locations from the study set (foils). Participants are instructed to identify as quickly as possible which combinations of person and location were in the study set and which were not. 
The fan of a concept is the number of different sentences in the study set that contained that concept. For example, if "the hippie is in the park" is the only sentence in the study set that mentions the hippie, then hippie has a fan of one. If participants learn that there are four people in the park during the study phase, then park has a fan of four.

The fan effect refers to the finding that participants are slower to recognize or reject sentences that contain concepts that have a higher fan. The more people in the park, the slower participants are to decide if the phrase "hippie is in the park" was in the study set. Likewise, if participants learn that the hippie is in several different locations, they are slower to decide if the hippie was in a particular location.

The fan effect illustrates a fundamental principle of human memory: the availability of a piece of information in memory with respect to a cue is a function of the probability of that piece of information conditional on the cue. If the participants learn four facts about the park, then given the cue park, each of those facts have only one chance in four of being the relevant fact to retrieve. The retrieval time from memory will reflect that one in four chance. Conversely, if the participants know only one fact about the park, given the cue park, retrieval time will be rapid, reflecting the $100 \%$ chance that the fact will be relevant.

\subsection{ACT-R's Declarative Memory (DM)}

In ACT-R, knowledge is represented in Declarative Memory (DM) as lists of slot:value pairs called chunks. Each slot is a task-relevant dimension of the stimulus, such as "colour" or "location". For example, a red square could be described by the chunk "colour:red shape:square". In the fan effect task, each sentence is represented by a chunk, e.g., 
"person:hippie place:park". In Python ACT-R, chunks can also be ordered lists of values without slots, "red square" or "hippie park". When the slots are omitted from a chunk, the order of the values in the chunk is used as the organizing principle.

Each chunk in DM has an activation. According to Anderson's (1991) rational analysis, the activation of a chunk in memory is an estimate of the likelihood of the information in the chunk being useful in the current situation. Given a cue that describes the current situation, ACT$\mathrm{R}$ retrieves the chunk in DM with the highest activation. Activation is a sum of a base level activation and a measure of the similarity between the chunk and the cue. Base level activation is a measure of how frequently and how recently the chunk has been used. For a chunk $i$, the activation of that chunk, $A_{i}$, is

$$
A_{i}=B_{i}+\sum_{j=1}^{n} W_{j} S_{j i}
$$

where $B_{i}$ is the baseline activation of the chunk, $n$ is the number of slot-value pairs in the cue, $W_{j}$ is the attention paid to slot-value pair $j$ of the cue, and each $S_{j i}$ is an association strength: a measure of the probability that chunk $i$ is relevant given that the cue contains slot-value pair $j$.

DM can be understood by analogy to a hydraulic system. Activation is like water and connections between cues and chunks are like pipes. Activation spreads from the cue to the chunks in DM. Chunks with stronger associations to the cue receive more activation. The chunk that receives the most activation is selected and retrieved from memory. The time, $T$, to retrieve a chunk, $i$, is a function of the chunk's activation, $A_{i}$, and two fitting parameters $I$ and $F$, 


$$
\text { (7.2) } \quad T=I+F e^{-A_{i}}
$$

The higher the activation, the shorter the retrieval time.

Although ACT-R has a mechanism for learning the association strengths, this has not been tested with the fan effect. Instead, each $S_{j i}$ for chunk $i$ and slot-value $j$ is

$$
S_{j i}=\mathrm{S}+\ln (\mathrm{P}(i \mid j))
$$

where $S$ is a fitting parameter and $\mathrm{P}(i \mid j)$ is the probability that chunk $i$ will be useful given the presence of the concept $j$ in the cue. In the fan effect, the chunk $i$ might be "hippie park" and $j$ might be park. If there are four people in the park then park has a fan of four. The probability that "hippie park" is the correct chunk given park is then $1 / 4$ or, more generally, $1 / f$ where $f$ is the fan.

In the fan effect task, the experimental design is supposed to control for frequency and recency effects, and so the ACT-R model of the fan effect assumes all chunks have the same baseline activation, $B_{i}$, and thus baseline activation can be removed from the equation. In Anderson and Reder's (1999) ACT-R model of the fan effect, reaction time for correctly identifying a target as belonging to the study set is calculated in milliseconds with the parameters $S=1.45, W_{j}=1 / 3, I=845$, and $F=613$. Using these parameters and simplifying Equations 7.1 and 7.2, we find that the target retrieval time for the fan effect model works out to be a function of the product of the person and place's fans: 


$$
T=233\left(f_{\text {person }} f_{\text {place }}\right)^{1 / 3}+845
$$

where $f_{\text {person }}$ is the person's fan and $f_{\text {place }}$ is the place's fan. This model provides a good fit to participant reaction times to targets in the fan effect task, $r=0.95$ (see Figure 7.1).

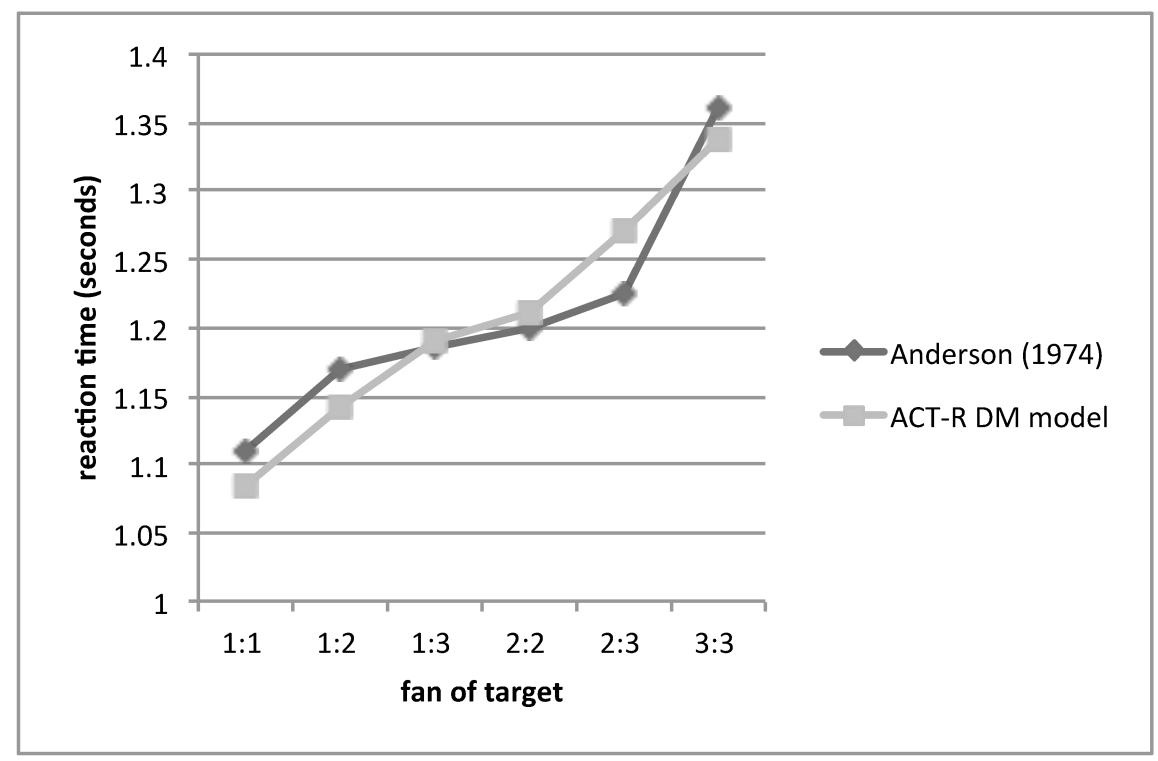

Figure 7.1. Real versus simulated reaction times for the fan effect from Anderson's (1974) data and Anderson and Reder's (1999) ACT-R DM model.

\subsection{Holographic Declarative Memory (HDM)}

In Anderson and Reder's (1999) ACT-R DM model of the fan effect, it is necessary to set the correct association strength $S_{j i}$ for each concept $j$ and chunk $i$. However, Holographic Declarative Memory (HDM) produces the fan effect by learning the study set. The association strengths are not explicitly programmed. The studied items are presented to HDM as ACT-R chunks. HDM uses holographic reduced representations (Plate, 1995), a technique for instantiating and 
manipulating symbolic structure in high-dimensional vectors. To interface with ACT-R, HDM translates chunks into vectors, and vectors into chunks.

In HDM, a value is represented by a vector of $n$ numbers randomly sampled from a normal distribution. These randomly generated vectors are referred to as environment vectors. Any two vectors chosen at random in a high dimensional space will tend to be approximately orthogonal. In HDM, angles indicate degrees of similarity. Orthogonality indicates complete dissimilarity. If we wanted to represent values with intrinsic similarity (e.g., brother and sister) we could choose non-orthogonal vectors, but for the purposes of modelling the fan experiment, we assume that the persons and locations are dissimilar.

In HDM, a slot is represented by a random permutation: a randomly selected reordering of a vector's elements. A slot-value pair is represented by reordering the elements of the value vector by the slot permutation.

Information storage in HDM is based on BEAGLE (Jones \& Mewhort, 2007) and DSHM (Rutledge-Taylor et al., 2014). HDM is a concept-based memory system. Rather than storing chunks per se, HDM stores relationships between concepts, i.e., the values from an ACT-R chunk. Each concept is represented by two vectors: an environment vector $\mathbf{e}_{\text {concept }}$ that represents the percept of that concept, and a memory vector $\mathbf{m}_{\text {concept }}$ that stores the relationship between that concept and other concepts.

As information storage in HDM differs from DM, so too does the process of retrieval. To recall from DM, DM is given a retrieval cue that is a description of a chunk and DM retrieves a chunk that matches that description. Conversely, in HDM, a cue is a question, represented by a vector, and HDM retrieves the concept that best answers that question. 


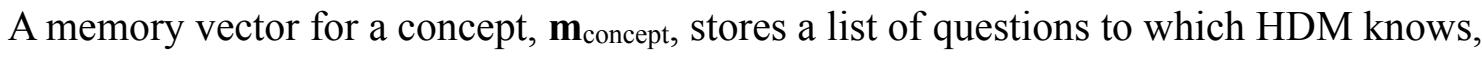
from experience, that the concept is a candidate answer. When cued, that is, posed a question, HDM selects the memory vector with the greatest similarity to the cue and gives as answer the concept represented by that memory vector.

In Python ACT-R, a cue may contain the value question mark, '?', to indicate a 'wildcard', that is, an unknown value. DM can retrieve more than one unknown value at a time because it is retrieving a complete chunk. Whereas in HDM, each unknown value requires a separate retrieval because HDM retrieves a value rather than a chunk (though we are open to the possibility that these retrievals could be performed in parallel). A chunk used as a cue for recall in HDM must contain exactly one '?' to indicate the concept (i.e., value) to be retrieved.

\subsection{Memory Encoding and Recall with Slots}

In HDM, there are two ways to structure knowledge corresponding to the two kinds of chunk in Python ACT-R: lists of values or unordered lists of slot-value pairs. We first discuss storing unordered slots-value pairs in HDM.

To store in HDM the chunk "colour:red shape:square size:large", we update the memory vector for each concept in the chunk: $\mathbf{m}_{\text {red }}, \mathbf{m}_{\text {square, }}$ and $\mathbf{m}_{\text {large. }}$ To update the memory vector for red, $\mathbf{m}_{\text {red, }}$ we need to construct a vector representing the relationship between the concept red and all other concepts in the chunk and then add that vector to $\mathbf{m}_{\text {red. }}$ In other words, we need to describe the set of questions for which red is an appropriate answer given "colour:red shape:square size:large" and add those questions to $\mathbf{m}_{\text {red. }}$ Those questions are "What colour is 
it?", "What colour is the large thing?", "What colour is the square?" and "What colour is the large square?".

The question "What colour is it?" can be represented by the chunk "colour:?", "What colour is the large thing?" by the chunk "colour:? size:large", and "What colour is the large square?" by "colour:? size:large shape:square".

When the cue is translated into a vector, the '?' becomes the placeholder (Jones \& Mewhort, 2007). The placeholder, denoted by $\boldsymbol{\Phi}$, is a vector used to encode all associations and thus serves as a universal retrieval cue. The placeholder is randomly generated like an environment vector. Using the placeholder, the cue "colour:?" is translated into the vector qcolour:? $=\left(\mathbf{P}_{\text {colour }} \boldsymbol{\Phi}\right)$, where $\mathbf{P}_{\text {colour }}$ is the permutation representing the slot colour.

In holographic reduced representations (Plate, 1995), there are two ways of combining a pair of vectors to create a new vector: + vector addition and * circular convolution. An association between concepts is represented by convolving together the environment vectors representing those concepts. Addition is used to superimpose vectors representing separate information into a single vector.

The vector that represents the question "colour:? size:large" is $\left(\mathbf{P}_{\text {colour }} \boldsymbol{\Phi}\right) *\left(\mathbf{P}_{\text {size }} \mathbf{e}_{\text {large }}\right)$, i.e., the placeholder permuted by colour and convolved with large permuted by size. This vector will only match the memory vectors of concepts that are colours associated with large objects.

By default, HDM allows for partial matching of cues to concepts in memory. To do so, HDM translates each cue into a set of questions: the question explicitly specified by the cue and all less specific variants of that question. The cue "colour:? size:large shape:square" is translated 
into a sum of vectors representing "colour:? size:large shape:square" and also "colour:? size:large", "colour:? shape:square”, and “colour:?", calculated as follows:

$$
\begin{aligned}
& \mathbf{q}_{\text {colour:? size:large shape:square }}= \\
& \quad\left(\mathbf{P}_{\text {colour }} \boldsymbol{\Phi}\right) \\
& +\left(\mathbf{P}_{\text {colour }} \boldsymbol{\Phi}\right) *\left(\mathbf{P}_{\text {size }} \mathbf{e}_{\text {large }}\right) \\
& +\left(\mathbf{P}_{\text {colour }} \boldsymbol{\Phi}\right) *\left(\mathbf{P}_{\text {shape }} \mathbf{e}_{\text {square }}\right) \\
& +\left(\mathbf{P}_{\text {colour }} \boldsymbol{\Phi}\right) *\left(\mathbf{P}_{\text {shape }} \mathbf{e}_{\text {square }}\right) *\left(\mathbf{P}_{\text {size }} \mathbf{e}_{\text {large }}\right)
\end{aligned}
$$

Cues are used both to retrieve from memory and to add new knowledge to memory. When the chunk "colour:red size:large shape:square" is added to memory, HDM updates $\mathbf{m}_{\text {red, }}, \mathbf{m}_{\text {square, }}$ and $\mathbf{m}_{\text {large }}$ as follows:

$$
\begin{aligned}
& \Delta \mathbf{m}_{\text {red }}=\mathbf{q}_{\text {colour:? size:large shape:square }} \\
& \Delta \mathbf{m}_{\text {square }}=\mathbf{q}_{\text {colour:red size:large shape: }} \\
& \Delta \mathbf{m}_{\text {large }}=\mathbf{q}_{\text {colour:red size:? shape:square }}
\end{aligned}
$$

Given a retrieval cue, HDM selects the memory vector with the greatest similarity to the cue's vector and the cue's chunk is returned to ACT-R with the '?' substituted for the concept that the memory vector represents.

Similarity is measured by the cosine of the angle between vectors, which can be calculated as: 


$$
\operatorname{cosine}(\mathbf{q}, \mathbf{m})=(\mathbf{q} \cdot \mathbf{m}) /\left((\mathbf{q} \cdot \mathbf{m})^{0.5}(\mathbf{q} \cdot \mathbf{m})^{0.5}\right)
$$

where $\mathbf{q}$ is a cue vector, $\mathbf{m}$ is a memory vector, and $\bullet$ is the dot product. The cosine is the dot product normalized by the magnitudes of the vectors. A cosine of 1 means the vectors are identical and 0 means they are completely dissimilar. HDM uses DM's retrieval time equation (Equation 7.2), but calculates activation as similarity measured by the cosine.

\subsection{Vectors without Slots}

Without slots, relationships are indicated by the order of the values in the chunk. Convolution is commutative, $\mathbf{a} * \mathbf{b}=\mathbf{b} * \mathbf{a}$, so the order is not preserved. To preserve the order we use $\mathbf{P}_{\text {before, }}$ a random permutation indicating that a vector occurred before another a vector. To add the chunk "large red square" to memory, we would update $\mathbf{m}_{\text {red }}, \mathbf{m}_{\text {square}}$, and $\mathbf{m}_{\text {large. }}$ We would update $\mathbf{m}_{\text {red }}$ as follows:

$$
\begin{aligned}
\Delta \mathbf{m}_{\text {red }}= & \left(\mathbf{P}_{\text {before }} \mathbf{e}_{\text {large }}\right) * \boldsymbol{\Phi} \\
& +\left(\mathbf{P}_{\text {before }} \boldsymbol{\Phi}\right) * \mathbf{e}_{\text {square }} \\
& +\left(\mathbf{P}_{\text {before }}\left(\left(\mathbf{P}_{\text {before }} \mathbf{e}_{\text {large }}\right) * \boldsymbol{\Phi}\right) * \mathbf{e}_{\text {square }}\right.
\end{aligned}
$$

which adds "large ?", "? square" and "large ? square" to the memory of the concept of red. 


\subsection{Recognition with Holographic Declarative Memory}

In the DM model of the fan effect, the activation of a chunk is calculated as a weighted sum of the association strengths of the chunk's constituent concepts. In HDM, association strengths are measured by vector cosine, so we can calculate that activation in HDM as a weighted sum of cosines.

When determining whether HDM recognizes a cue, the cue chunk must contain no unspecified values '?'. For each value in the cue, HDM creates a new cue with that value substituted for '?', performing one retrieval for each value in the original cue. Activation is calculated as the mean of the cosines between each of these cues and the memory vector of the concept that was substituted out to create the cue. This method for calculating activations in the fan effect has been used before by DSHM (Rutledge-Taylor et al., 2014; Rutledge-Taylor, Pyke, West, \& Lang, 2010).

In the fan effect task, for the cue "hippie park", HDM does two retrievals, "hippie ?" and

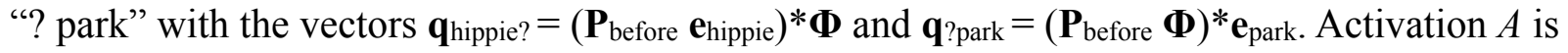
calculated as:

$$
A=0.5 \operatorname{cosine}\left(\mathbf{q}_{\text {hippie? }}, \mathbf{m}_{\text {park }}\right)+0.5 \operatorname{cosine}\left(\mathbf{q} \text { ?park }, \mathbf{m}_{\text {hippie }}\right)
$$

\subsection{The HDM Model of the Fan Effect}

We ran the HDM model of the fan effect task 20 times, simulating 20 virtual participants, and averaged across runs. Because each run uses a different set of random vectors, the cosines and reaction times vary randomly with each run. Anderson's (1974) experiment had 18 participants. 
The model fits the human participant data reported by Anderson (1974) with a correlation of $r=$ 0.91 (see Figure 7.2). The fit was obtained using the exact same values for the fitting parameters as Anderson and Reder's (1999) ACT-R fan effect model. The only change was to compute activation as a mean of cosines, as described in the previous section.

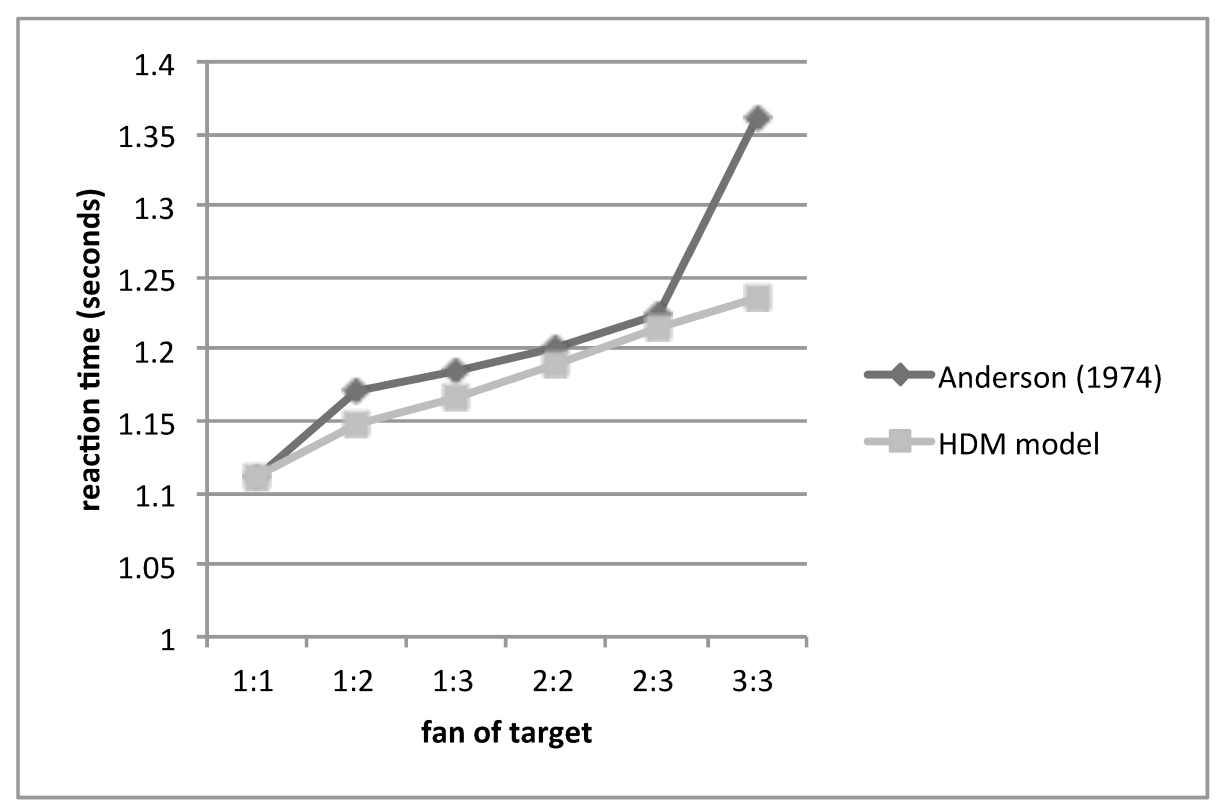

Figure 7.2. Real versus simulated reaction times for the fan effect from Anderson's (1974) data and the HDM model.

Anderson and Reder's (1999) model and the HDM model are strongly correlated, $r=$ 0.99. While there are slight differences in the predictions made by the two models, both the DM and HDM models are within the range of human variability for performance on this task. These results show that HDM replicates DM's ability to model the fan effect, but HDM does so in a radically different way: by measuring the cosine between vectors in a high-dimensional space.

Why does the cosine model the fan effect so well? The cosine acts as an estimate of the conditional probabilities that the Anderson and Reder's (1999) fan effect model uses to compute 
association strengths. The memory vector for a concept keeps a fuzzy count of the number of times that concept has co-occurred with each other concept. Taking the dot product of the cue with a memory vector gives you an estimate of the frequency with which that cue has been added to that memory vector, that is, the number of times the relationships described in that cue have occurred with that concept. The cosine is a dot product normalized by the magnitudes of the vector, which in this case, is a frequency normalized by the total number of instances, that is to say, the cosine is roughly the probability.

We can imagine all vectors in HDM as points on a $n$-dimensional hypersphere. For the HDM fan effect model, we used 256 dimensions, but for the sake of visualization, imagine a 3dimensional sphere.

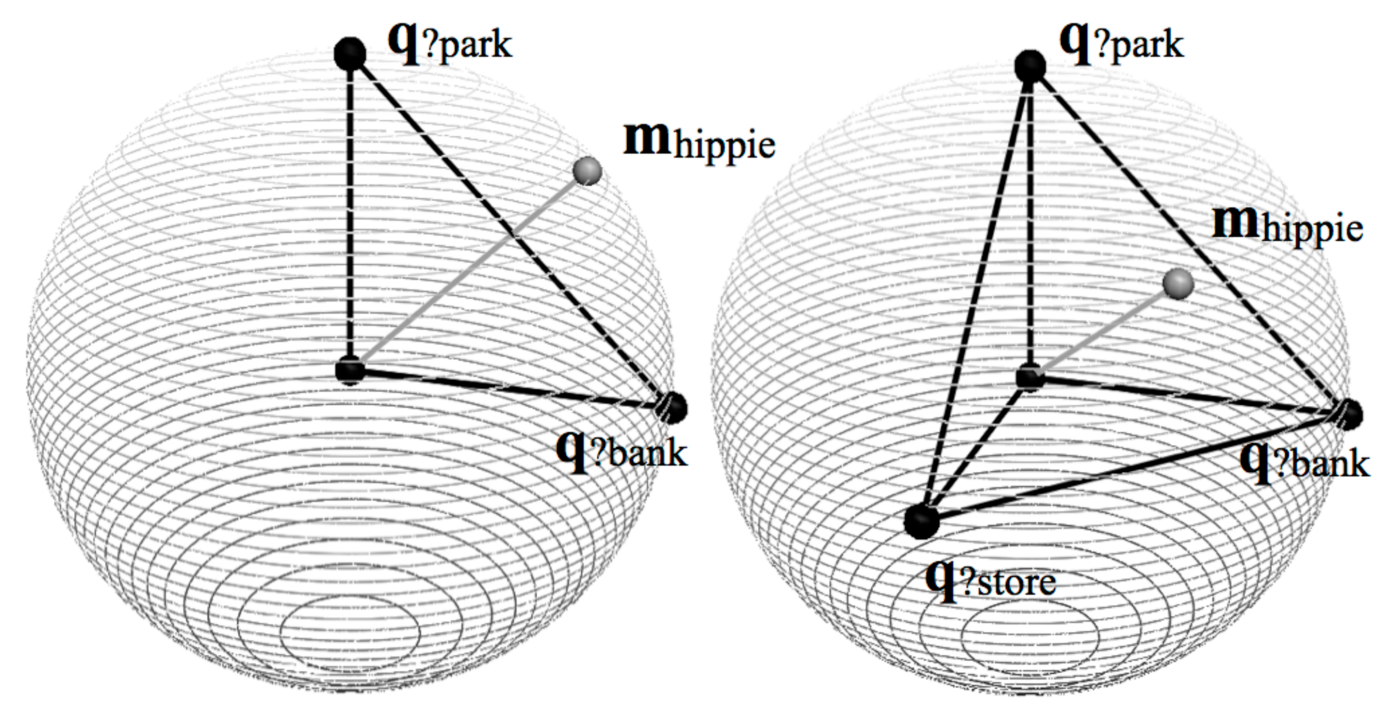

Figure 7.3. $\mathbf{m}_{\text {hippie }}$ with a fan of 2 (left) or 3 (right).

Let us first consider a fan of one. Suppose the model has learned only one fact about the hippie, namely, the "hippie is in the park". After learning this fact, the memory vector for hippie 
will be $\mathbf{m}_{\text {hippie }}=\left(\mathbf{P}_{\text {before }} \boldsymbol{\Phi}\right) * \mathbf{e}_{\text {park. }}$. The model is later given the cue "the hippie is in the park" during the recognition phase. To test for recognition, we take the cosine of $\mathbf{m}_{\text {hippie }}$ with the cue $\mathbf{q}_{\text {? }}$ park $=\left(\mathbf{P}_{\text {before }} \boldsymbol{\Phi}\right) * \mathbf{e}_{\text {park. }}$. As $\mathbf{m}_{\text {hippie }}=\mathbf{q}_{\text {?park }}$ the angle between the cue and the memory vector is zero, the distance between them on the surface of the hyper-sphere is zero, and the cosine is 1.00 .

Let us consider a fan of two. If the model knows "hippie is in the park" and "hippie is in the bank", then $\mathbf{m}_{\text {hippie }}$ is the sum of the park cue $\mathbf{q}$ ?park and the bank cue $\mathbf{q}$ ?bank,

$$
\mathbf{m}_{\text {hippie }}=\left(\mathbf{P}_{\text {before }} \boldsymbol{\Phi}\right) * \mathbf{e}_{\text {park }}+\left(\mathbf{P}_{\text {before }} \boldsymbol{\Phi}\right)^{*} \mathbf{e}_{\text {bank }}
$$

In high dimensional spaces, randomly chosen vectors are approximately orthogonal to each other. Let us assume that the cues $\mathbf{q}_{\text {?bank }}$ and $\mathbf{q}_{\text {?park }}$ are perfectly orthogonal. As illustrated in the left half of Figure 7.3, on the surface of the hypersphere, $\mathbf{m}_{\text {hippie }}$ will be halfway between the two cues at a $45^{\circ}$ angle. The cosine is 0.71 .

Let us consider a fan of three. As illustrated in the right half of Figure 7.3, if the model knows that the hippie is in the bank, park, and store, $\mathbf{m}_{\text {hippie }}$ will be at an equidistant point on the hypersphere between the cues for bank, park, and store. In a fan of three, $\mathbf{m}_{\text {hippie }}$ is further from all the cues than in a fan of two. The angle from $\mathbf{m}_{\text {hippie }}$ to any cue is $55^{\circ}$ and the cosine is 0.58 .

Where $f$ is the fan, the cosine between a cue and a memory vector is $f^{-1 / 2}$ if the vectors are perfectly orthogonal, or approximates $f^{-1 / 2}$ for the random vectors used by HDM. Thus HDM predicts that as the fan increases, the cosine decreases, but by diminishing amounts with each increase in fan. As the fan approaches infinity, the cosine approaches zero. HDM makes the 
intuitive prediction that increases in the fan has a steadily diminishing effect on reaction time, such that knowing 100 facts about the hippie is not appreciably different from knowing 101.

The cosine in HDM approximates the square-root of the probability only when the events are equiprobable. For $n$ events with frequencies $v_{1}$ to $v_{n}$, the cosine of event $i$ is

$$
\text { cosine }=\frac{v_{i}}{\sqrt{v_{1}^{2}+\ldots+v_{i}^{2}+\ldots v_{n}^{2}}}
$$

When given events of unequal probabilities, HDM will behave as if the most frequent events are disproportionately likely and the least frequent events are disproportionately unlikely. This is a testable and possibly erroneous prediction of HDM. The quantum probability model of human judgements (Busemeyer, Pothos, Franco, \& Trueblood, 2011) also uses vector algebra to calculate probabilities, but uses the square-roots of the frequencies, then squares the cosine, such that Equation 7.3 is equal to classical probability. Using the square-roots of the frequencies is not possible for HDM as it requires HDM to know a priori how frequently each event will occur.

On the basis of this analysis, we note that HDM can be made to mimic the behaviour of Anderson and Reder's (1999) ACT-R DM model of the fan effect exactly if, instead of substituting the activation for a mean of cosines, we use ACT-R DM's activation equation, but substitute the $S_{j i}$ term for the square of the cosine. As long as the events are equiprobable, the square of the cosine will be exactly $\mathrm{P}(i \mid j)$. 


\subsection{Future Work and Applications of HDM}

We have presented in this chapter an HDM model of the fan effect and compared it to Anderson and Reder's (1999) DM model of the fan effect. However, we have only discussed fitting to the reaction time of targets, sentences presented at the recognition phase that occurred in the study set. Anderson and Reder's (1999) model for foils, sentences that were not in the study set, fails on a variant of the fan effect task (West, Pyke, Rutledge-Taylor, \& Lang, 2010). As the foil is difficult to model, we leave developing an HDM model of the foil for future research.

At present, HDM does not model recency effects, that is, more recent information is not recalled better than less recent information. However, other holographic models in the literature (e.g., Franklin \& Mewhort, 2015; Murdock 1993) can account for recency effects, so such a mechanism could be incorporated into the model.

At present, interfacing with ACT-R chunks imposes an information bottleneck on HDM. Detailed sensory information cannot be feasibly stored in ACT-R chunks, but can be stored in holographic vectors (Kelly, Blostein, \& Mewhort, 2013). Reimplementing the entirety of ACT-R as a holographic system would improve ACT-R's ability to interface with real world environments and to match situations to procedures. Some of that work has already been done: A holographic model similar to ACT-R's procedural memory system already exists as the basal ganglia model of the SPAUN brain model (Eliasmith, 2013; Stewart, Bekolay, \& Eliasmith, 2012). However, a holographic procedural memory consistent with HDM and ACT-R would necessarily differ from SPAUN's to meet the demands of integration with a different architecture.

HDM is a powerful tool for cognitive modellers because it inherits the abilities of holographic models such as BEAGLE (Jones \& Mewhort, 2007) and DSHM (Rutledge-Taylor, 
Vellino, \& West, 2008) to store large quantities of data in memory and use it to make intelligent predictions in knowledge-heavy tasks. In the next chapter, we show that HDM can be used to model a difficult but small-scale decision-making task. In the future, HDM could be applied to a large-scale, knowledge-driven decision-making task.

\subsection{Conclusion}

We present a new module for ACT-R, Holographic Declarative Memory (HDM). We substitute HDM for DM in the ACT-R model of the fan effect and find that without changing any parameters HDM provides a good fit to the fan effect. We present an analysis that allows us to specify the mathematical relationship between the DM and HDM models of the fan effect.

We find that HDM accounts for the fan effect because the vector algebra of the model computes an estimate of the conditional probability of the target given the probe. HDM implements a general principle of cognition: the availability of a piece of information in memory is an estimate of its likelihood to be relevant to the current situation. Thus, HDM can be understood as a vector implementation of Anderson's (1991) rational analysis of memory. This analysis shows that HDM is closely related to quantum models of human probability judgements (Busemeyer et al., 2011), which also use vector algebra to model cognitive probability estimates. Because HDM models the fan effect using general memory principles, we expect HDM to do well modelling other memory tasks.

HDM, by virtue of being a holographic model, has a number of capabilities for which DM is less suited, such as analogical or case-based reasoning, learning associations between concepts without having association strengths set by the modeller, and performing tasks that 
require large amounts of knowledge. We hope that by integrating a holographic memory model into ACT-R, we can bring the capabilities of vector space modelling into the ACT-R research community and enhance the capability of the ACT-R cognitive architecture to model human cognition. In turn, we hope to use ACT-R to provide a platform for integrating models of memory with other cognitive systems for the sake of developing better cognitive models. 


\section{Chapter 8 Procedural Learning and Decision Making}

The ability to detect and predict patterns is an integral part of memory models and an important part of decision making. However, decision making also requires the ability to evaluate states as more or less desirous in order to motivate the decisions. We apply HDM to the decision-making task of Walsh and Anderson (2011). By initializing memory to a state of optimism and by making the model sensitive to dependencies between non-consecutive events, we find that the model is able to learn the task at a rate similar to humans.

\subsection{Introduction}

Here we apply HDM to a binary decision-making task where the model must learn from delayed, probabilistic feedback.

While memory models can be powerful tools for detecting structure within data and making predictions from experience, memory models are generally only used to make very simple decisions, such as deciding whether a stimulus is familiar or novel in a familiarity task, or when to 'give up' trying to recall more items in a free recall task.

Conversely, cognitive architectures such as ACT-R and Soar (Laird, 2012) are designed to make complicated sequences of decisions to solve problems. ACT-R uses two systems: a procedural memory and a declarative memory. Procedural memory consists of condition-action rules weighted by utility scores. Declarative memory consists of chunks of associated data weighted by activation values. 
Both the activation of a chunk in declarative memory and the utility of a condition-action rule in procedural memory can be understood as estimates of the usefulness of the chunk or rule. According to Anderson's (1991) rational analysis, the activation of a chunk in memory is an estimate of the likelihood of the information in the chunk being useful in the current situation. Likewise, a production rule's utility is an estimate of the usefulness of performing the rule's action. However, these two kinds of usefulness are quite distinct. For example, it is very useful to know that touching a hot object will burn you, but it is not very useful to touch a hot object. Thus the knowledge of the consequences of touching a hot object will have a high activation in declarative memory but the utility of touching hot objects as a rule in procedural memory will be low.

More generally, the probability of an event is distinct from the utility of the event. Thus, while computational models of human memory are well-equipped to estimate probabilities, they are not designed for decision making because they do not estimate utility.

Adapting HDM to decision making is attractive because the complexity and intelligence of human decision making is thanks, at least in part, to the immense quantity of experiential knowledge that humans draw upon. A HDM decision-making model may be able to scale up to decision-making tasks that require a great deal of knowledge.

In what follows, we model human performance in a sequential decision task. This task has already been modelled as a utility learning task by Walsh and Anderson (2011) using temporal difference learning models that can be incorporated into the ACT-R procedural memory. We instead model the task as a prediction task using HDM. 
Our approach to modeling decision making is related to the way DSHM models play Paper, Rock, Scissors (Rutledge-Taylor et al., 2014) and is conceptually similar to ACT-R models that use declarative, rather than procedural, memory to make decisions (e.g., Lebiere, Gonzalez, \& Martin, 2007).

\subsection{Experiment}

We summarize the pertinent details of the Walsh and Anderson (2011) experimental task here. Figure 8.1 illustrates the structure of a single trial of the experiment. Boxes are states in the experiment. Arrows indicate transitions. Thicker arrows indicate more probable transitions. Unlabelled arrows have a probability of $100 \%$.

In each trial of the experiment, participants make two choices that affect their chance of receiving positive feedback at the end of the trial. At the start of each trial, participants were presented with their first choice: a pair of letters, one on the left of the screen and one on the right. By pressing the corresponding arrow key, participants selected either the left or right letter. After their selection, a cue shape flashed onto the screen for over a second. Participants then selected a second letter from a different pair of letters. After their second choice, one of two symbols appeared indicating either positive or negative feedback. This ended the trial after which a new trial would begin.

The position of a letter on either the right or left side of the screen was randomized from one trial to the next, ensuring that participants could not rely on learning a pattern of motor responses to perform the task. 
Additionally, the particular letters, shapes, and symbols used in the task were randomized across participants to control for any effect of the symbols themselves.

However, for the purposes of discussing the task, we will use the letters $\mathbf{R}$ and $\mathbf{J}$ to represent the first binary choice, $\mathbf{I}$ and $\checkmark$ to represent the pair of shapes used as cues, $\mathbf{T}$ and $\mathbf{V}$ to represent the second binary choice, and we will refer to the pair of symbols that represent positive and negative feedback as good and bad.

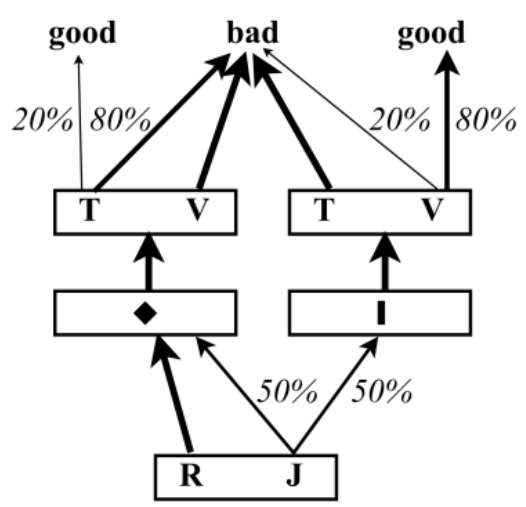

Figure 8.1. Experimental states and transition probabilities. Adapted from Figure 9.2 in Walsh \& Anderson (2011).

As illustrated in Figure 8.1, there are three distinct decisions to be made by the participant. For each decision, there is a correct choice, that is, a choice that maximizes the chance of receiving positive feedback. Between $\mathbf{R}$ and $\mathbf{J}, \mathbf{J}$ is correct. After $\bullet, \mathbf{T}$ is correct, whereas after $\mathbf{I}, \mathbf{V}$ is correct.

Walsh and Anderson (2011) measured participant response accuracy after 200 and 400 trials. Additionally, Walsh and Anderson used scalp-recorded event-related potential (ERP) to detect feedback-related negativity, a hypothesized neural correlate of prediction error. We 
attempt to model only response accuracy. We believe that prediction error, or surprise, could be modeled using an HDM-like model, as we discuss in Chapter 10.

\subsection{The Model}

Presentation of the experimental task is simplified for the model. In this simplified format for the experiment, there are nine distinct symbols. There is a symbol that indicates the start of a trial (which we will refer to as start), two letters that represent the first binary choice ( $\mathbf{R}$ and $\mathbf{J}$ ), a

pair of shapes used as cues ( $\mathbf{I}$ and $\bullet$ ), a second pair of letters used to represent the second binary choice ( $\mathbf{T}$ and $\mathbf{V}$ ) and a pair of items, good and bad, to represent positive and negative feedback.

At the beginning of the experiment, the model generates an item for each of the nine symbols used in the experiment. In the model runs discussed in this chapter, each vector has 1024 dimensions.

The memory vectors for each item are initialized, in a non-standard manner, to a state of optimism, such that the model initially believes that any symbol could be followed by good. Noise is added to the memory vectors to create random variation in the initial choices made by the simulated participants. The initialization of the memory vectors is explained in detail under subheading Initializing memory to a state of optimism. The memory vectors are used to make decisions and are updated after every iteration over the course of the experiment.

The experiment is run for 400 trials. Each trial consists of five iterations as illustrated in Table 8.1. On the first iteration in a trial, the start symbol appears and the model must select $\mathbf{R}$ or $\mathbf{J}$. On the second iteration, the selected letter appears. On the third iteration, a cue appears 
(either $\bullet$ or $\mathbf{I}$ ) and the model must select either $\mathbf{T}$ or $\mathbf{V}$. On the fourth iteration, the selected letter appears. On the fifth iteration, either good or bad appears and then a new trial begins.

The model keeps track of what symbols have occurred so far in the current trial and the order in which those symbols occurred. This information can be understood as being held in a working memory buffer, but how this information is held is not a theoretical commitment of the model.

Table 8.1. The symbols presented to the model and choices available at each iteration in a trial.

\begin{tabular}{lccccc} 
Iteration & 1 & 2 & 3 & 4 & 5 \\
\hline Presented & start & $\mathbf{R}$ or $\mathbf{J}$ & $\bullet$ or $\mathbf{T}$ & $\mathbf{T}$ or $\mathbf{V}$ & $\begin{array}{c}\text { good } \\
\text { or bad }\end{array}$ \\
Choices & $\mathbf{R}$ or $\mathbf{J}$ & & $\mathbf{T}$ or $\mathbf{V}$ & &
\end{tabular}

On each iteration of the experiment, the model updates the memory vectors corresponding to each symbol that has appeared so far in the current trial. If, on the third iteration of a trial, the symbols presented so far are "start $\mathbf{J} \diamond$ ", then, on that iteration, the model will update the memory vectors for start, $\mathbf{J}$, and $\diamond$. If the model selects $\mathbf{T}$, then on the fourth iteration it will be presented the letter $\mathbf{T}$, and the memory vectors for start, $\mathbf{J}, \diamond$, and $\mathbf{T}$ will be updated. Memory vectors are updated with observed relationships between symbols, as detailed in Updating memory using open n-grams.

Decisions are made by selecting the choice that has the greatest similarity to the probe vector. The probe vector is constructed to ask the question, "Of the choices available, which choice is more likely to be followed by good?". This is detailed in Probing memory. 
Gradually, the model learns that for each choice, there is a better alternative. Learning happens for each of the three choices at roughly the same rate as human participants performing the task, as illustrated in the next section, Comparing the model to human performance.

Developing this model required addressing two questions that have not been previously addressed in DSHM or BEAGLE models on which HDM is based, namely, how to model motivation and how to learn dependencies between non-consecutive events. The question of modeling motivation is addressed in Representing positive and negative feedback and in Initializing memory to a state of optimism. The question of learning dependencies between nonconsecutive events is addressed in Updating memory using open n-grams.

\subsection{Comparing the model to human performance}

Walsh and Anderson (2011) had 13 participants in their experiment. Each participant performed two blocks of 400 trials. Walsh and Anderson present data aggregated across the 26 experimental blocks. Figure 8.2 depicts mean response accuracy with standard error indicated by error bars.

For the purposes of comparison, we average across 26 runs of the model. On each run, the model performs 400 trials. Comparing Figures 8.2 and 8.3, we see that performance of the model roughly mirrors human performance. Performance for both humans and the model is better in the latter half of the trials. Both humans and the model learn that $\mathbf{V}$ is the correct choice after $\mathbf{I}$ with relative ease, learn that $\mathbf{J}$ is a better choice than $\mathbf{R}$ more slowly, and have the most difficulty learning that $\mathbf{T}$ is the correct choice after $\bullet$.

The reliability with which the model learns to choose $\mathbf{T}$ after $\checkmark$ varies with a parameter, the optimism coefficient. This parameter is explained in the section Initializing memory to a state 
of optimism. The parameter encourages exploration, but if the value is too high, the model tends to explore too much and insufficiently exploit knowledge of the task gained by this exploration.

Figure 8.3 shows results for an optimism coefficient of 30 .

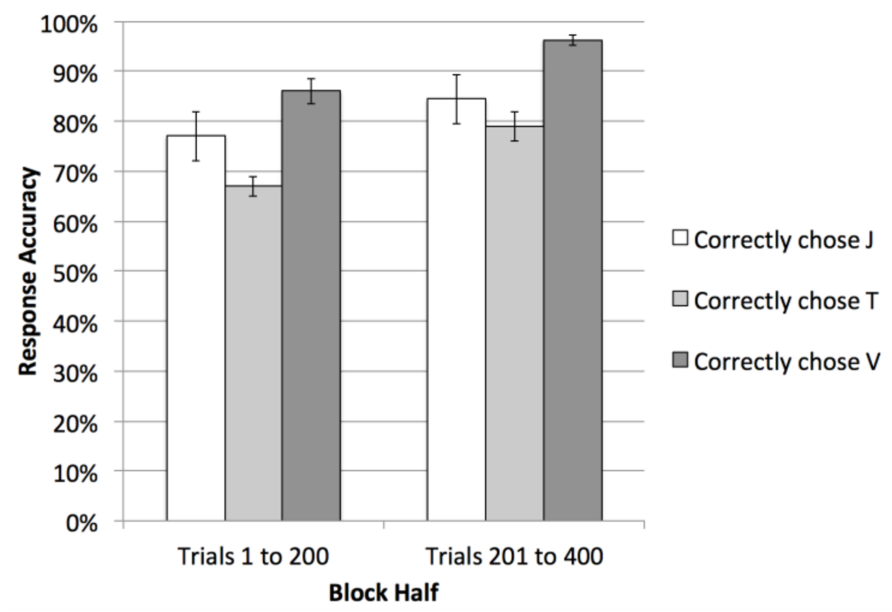

Figure 8.2. Response accuracy for human participants. Adapted from Figure 9.3 in Walsh \& Anderson (2011).

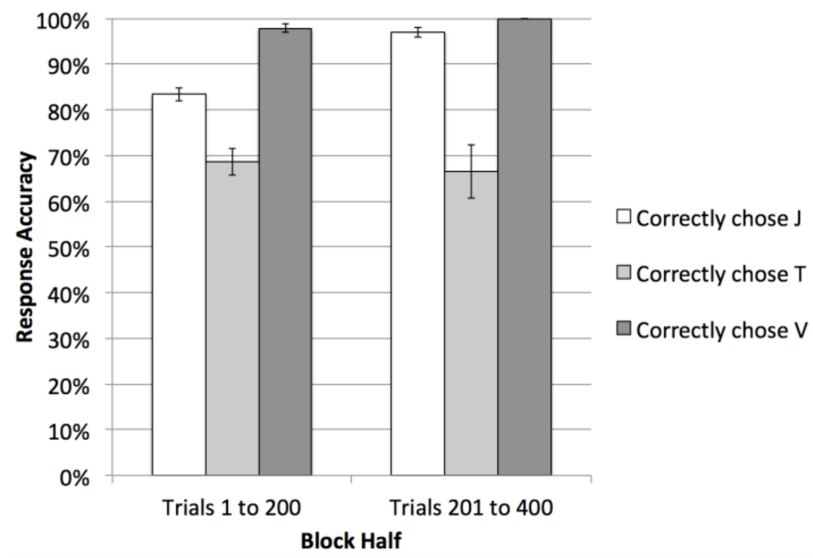

Figure 8.3. Response accuracy for the model.

While the model captures the overall pattern of human performance, there are two noteworthy differences. First, the model often learns to do the task nearly perfectly, whereas human 
participants do not tend to do so. The model's rate of correctly choosing $\mathbf{V}$ after $\mathbf{I}$ reaches ceiling in the first block half and so does not improve significantly in the second block half. However, the model's accuracy can be impaired by using vectors with far fewer dimensions.

Second, the model's mean rate of correctly choosing $\mathbf{T}$ after $\downarrow$ does not increase in the second block half. Improving the model's performance after $\bullet$ may require implementing an attentional mechanism to allow the model to learn that the relationship between $\checkmark$ and $\mathbf{T}$ is critical to the feedback received and thus needs to be weighted more heavily. Implementing an attentional mechanism is discussed in Probing memory.

\subsection{Representing positive and negative feedback}

In the experimental task, participants receive positive or negative feedback at the end of each trial. Positive feedback and negative feedback take the form of arbitrary symbols that the participant has been told indicate "1 point gained" or "no points gained" respectively. Participants in Walsh and Anderson's (2011) experiment were promised $\$ 1.00$ for every 50 points they gained.

Our best model makes no attempt to represent positive and negative feedback as being associated with positive or negative feelings. The model makes choices that maximize the likelihood of getting good to appear at the end of the trial. The model avoids choices that are likely to cause bad to appear simply because those choices are less likely to cause good to appear.

We have explored modeling the bad symbol as an aversive stimulus. This is implemented by setting the environment vector of bad to be the negation of the environment vector of good. 
We find that when bad is represented this way, the model becomes, as one might expect, risk averse and reluctant to explore possibilities. As a result, when bad is the negation of good, the model never learns that $\mathbf{T}$ yields a $20 \%$ chance of $\operatorname{good}$ after the cue $\bullet$. Instead, the model chooses $\mathbf{V}$ regardless of which cue has been presented because it quickly learns that $\mathbf{V}$ often yields good and is reluctant to try its chances with $\mathbf{T}$. We speculate that if humans were subjected to an aversive stimulus (e.g., an unpleasant image) every time they received negative feedback in this task, we might observe an impairment in problem solving performance similar to when bad is modelled as the negation of good.

Conversely, representing bad as a neutral stimulus encourages the model to try selecting options that have frequently yielded bad, which, when combined with optimism, leads to a more thorough exploration of the choices available within the task, allowing the model to better understand the consequences of its choices and discover the optimal pattern of decisions (see Figure 8.3).

The contrast between how the model behaves when bad is modelled as aversive versus neutral is consistent with broaden-and-build theory of positive emotions, which holds that positive emotions encourage exploration by broadening the repertoire of actions considered, whereas negative emotions narrow that repertoire (Fredrickson, 2001).

\subsection{Initializing memory to a state of optimism}

At the start of the experiment, memory vectors are initialized to a state of optimism. For all symbols in the task (except for good and bad), the model initially believes that symbol may later be followed by good. 
The purpose of optimism is to motivate the model to select untried (or infrequently tried) choices. Without optimism, the model would simply repeat the same choices over and over again, as the model has no reason to believe that the other options would be any better.

Lebiere et al. (2007) also encountered this problem in modeling decision-making and likewise decided to initialize their ACT-R model to a state of optimism.

Making bad an aversive stimulus is an alternative to optimism that motivates the model to avoid choices that yield bad. However, there are two disadvantages to this approach: (1) Human participants are not punished for incorrect choices: they are rewarded for correct choices, and so such a model is performing a different task than the task that humans are asked to perform, and (2) either with or without optimism, variants of the model that are punished for incorrect choices fail to learn that $\mathbf{T}$ is the correct choice after the cue, as discussed in the previous section.

Without optimism, the memory vector for a symbol is initialized to be equal to the environment vector for that symbol. This serves the purpose of adding random noise such that the initial decisions made by the model vary from one simulated participant (i.e., run of the model) to the next.

With optimism, an expression meaning "this is followed by good" is added to the memory vector when it is initialized. For any symbol $s$,

$$
\mathbf{m}_{s}=\mathbf{e}_{s}+a\left(\left(\mathbf{P}_{\text {before }} \boldsymbol{\Phi}\right) * \mathbf{e}_{\text {good }}\right)
$$


where $a$ is the optimism coefficient, a scalar value and parameter of the model. Figure 8.3 shows performance of the model for $a=30$.

\subsection{Updating memory using open $n$-grams}

In the experiment, the choice $\mathbf{J}$ is more likely to lead to good than the choice $\mathbf{R}$. However, there are two intervening events between feedback and the initial choice: the appearance of a cue and a second decision. In human memory, is a direct association formed between the first choice and the feedback at the end of the trial? Or do people rely on a chain of associations, learning that $\mathbf{J}$ leads to a particular cue, which in turn leads to a choice, which in turn leads to an increased probability of positive feedback?

With our model we have assumed that people form a direct association between their initial choice and the feedback received at the end of the trial. The memory update scheme used is a modification of the scheme used by BEAGLE (Jones \& Mewhort, 2007) and inspired by Hannagan, Dupoux, and Christophe (2011).

Hannagan et al. (2011) considered a variety of models for how letter position in words is encoded in memory and found that the encoding scheme, unconstrained open bigrams, provided a good model of the kinds of errors people typically make in spelling. In this scheme, all pairs of letters within a word are associated with each other, even pairs of letters that are not next to each other in the word.

In our scheme, at each iteration of a trial, all symbols presented so far in a trial are associated with each other and all groups of symbols are associated with each other, even if the symbols do not occur consecutively. 
For example, on the final iteration of a trial "start $\mathbf{R} \bullet \mathbf{T} \operatorname{good}$ " the memory vector for $\mathbf{R}$ is updated with,

$$
\begin{array}{lc}
\left(\mathbf{P}_{\text {before }} \boldsymbol{\Phi}\right) * \mathbf{e}_{\text {good }} & \text { i.e., "this came before } \mathbf{g o o d} " \\
+\left(\mathbf{P}_{\text {before }}\left(\mathbf{P}_{\text {before }} \boldsymbol{\Phi}\right) * \mathbf{e}_{\bullet}\right) * \mathbf{e}_{\text {good }} & \text { i.e., "this came before } \bullet \mathbf{g o o d} " \\
+\left(\mathbf{P}_{\text {before }}\left(\mathbf{P}_{\text {before }} \boldsymbol{\Phi}\right) * \mathbf{e}_{\mathbf{T}}\right) * \mathbf{e}_{\text {good }} & \text { i.e., "this came before } \mathbf{T} \mathbf{g o o d} " \\
+\left(\mathbf{P}_{\text {before }}\left(\mathbf{P}_{\text {before }}\left(\mathbf{P}_{\text {before }} \boldsymbol{\Phi}\right) * \mathbf{e}_{\diamond}\right) * \mathbf{e}_{\mathbf{T}}\right) * \mathbf{e}_{\text {good }} & \text { i.e., "this came before } \bullet \mathbf{T} \mathbf{g o o d} "
\end{array}
$$

$\mathbf{R}$ is also updated with "this came after start and came before good", "this came after start and came before $\diamond$ good", etc.

\subsection{Probing memory}

The probe vector is constructed to ask memory the question "Given what happened in the trial so far, of the choices available, which choice is more likely to be followed by good?". The probe vector is the sum of:

$$
\begin{aligned}
& \left(\mathbf{P}_{\text {before }} \mathbf{\Phi}\right) * \mathbf{e}_{\text {good }} \\
& +\left(\mathbf{P}_{\text {before }}\left(\mathbf{P}_{\text {before }} \mathbf{e}_{a}\right) * \boldsymbol{\Phi}\right) * \mathbf{e}_{\text {good }} \\
& +\left(\mathbf{P}_{\text {before }}\left(\mathbf{P}_{\text {before }}\left(\mathbf{P}_{\text {before }} \mathbf{e}_{b}\right) * \mathbf{e}_{a}\right) * \boldsymbol{\Phi}\right) * \mathbf{e}_{\text {good }} \\
& \ldots \text { etc. ... }
\end{aligned}
$$

i.e., this came before good; this came before good and after $a$; this came before good and after $b$ 
and $a$; etc. where $a$ is the symbol presented on this iteration, $b$ is the symbol presented on the previous iteration, and so on, back to the beginning of the current trial. For the first decision in a trial, the probe vector is simply:

$$
\left(\mathbf{P}_{\text {before }} \boldsymbol{\Phi}\right) * \mathbf{e}_{\text {good }}+\left(\mathbf{P}_{\text {before }}\left(\left(\mathbf{P}_{\text {before }} \mathbf{e}_{\text {start }}\right) * \boldsymbol{\Phi}\right)\right) * \mathbf{e}_{\text {good }}
$$

i.e, this came before good; this came after start and before good. For the second decision, the probe contains four terms, i.e.,

$$
\begin{aligned}
& \left(\mathbf{P}_{\text {before }} \boldsymbol{\Phi}\right) * \mathbf{e}_{\text {good }} \\
& +\left(\mathbf{P}_{\text {before }}\left(\mathbf{P}_{\text {before }} \mathbf{e} \text { or } \mathbf{I}\right) * \boldsymbol{\Phi}\right) * \mathbf{e}_{\text {good }} \\
& +\left(\mathbf{P}_{\text {before }}\left(\left(\mathbf{P}_{\text {before }} \mathbf{e}_{\mathbf{R} \text { or } \mathbf{J}}\right) * \mathbf{e} \text { or } \mathbf{I}\right) * \boldsymbol{\Phi}\right) * \mathbf{e}_{\text {good }} \\
& +\left(\mathbf{P}_{\text {before }}\left(\mathbf{P}_{\text {before }}\left(\mathbf{P}_{\text {before }}\left(\mathbf{P}_{\text {before }} \mathbf{e}_{\text {start }}\right) * \mathbf{e}_{\mathbf{R} \text { or } \mathbf{J}}\right) * \mathbf{e} \bullet \text { or } \mathbf{l}\right) * \boldsymbol{\Phi}\right) * \mathbf{e}_{\text {good }}
\end{aligned}
$$

Decisions are made by measuring the similarity, as calculated by vector cosine, between the probe vector and the memory vectors of the two available choices. The model selects as its choice the symbol corresponding to the memory vector with the highest similarity.

Note that for the purposes of this particular model, the only symbols that need to have memory vectors are the choice symbols, represented here as $\mathbf{R}, \mathbf{J}, \mathbf{T}$, and $\mathbf{V}$. However, it is a theoretical or architectural commitment of HDM that all perceptual stimuli have an associated memory vector. 
The probe vector is constructed to ask a distinct question for each term that comprises it. For the first decision, those questions are "Which of $\mathbf{R}$ or $\mathbf{J}$ is most likely to lead to $\mathbf{g o o d}$ ?" and "Given that the start symbol was just seen, which of $\mathbf{R}$ or $\mathbf{J}$ is most likely to lead to $\mathbf{g o o d}$ ?". For the second decision, four questions are posed by the probe: "Which of $\mathbf{T}$ or $\mathbf{V}$ is most likely to lead to good?", "Given the cue shape that was just seen, which of $\mathbf{T}$ or $\mathbf{V}$ is most likely to lead to good?", "Given the cue shape that was just seen and the previous decision that was made, which of $\mathbf{T}$ or $\mathbf{V}$ is most likely to lead to good?", and "Given the cue shape that was just seen, the previous decision that was made, and the fact that the start symbol was seen before that, which of $\mathbf{T}$ or $\mathbf{V}$ is most likely to lead to good?". The similarity of the probe vector to a particular choice's memory vector is an approximate estimate of the probability that choice will lead to good made on the basis of the model's experiences and optimism.

For example, in this experiment, $\mathbf{V}$ is, overall, very likely to be followed by good, but if $\mathbf{V}$ is preceded by the cue or the choice $\mathbf{R}, \mathbf{V}$ will not be followed by good. The one question in the probe that is answered with "very likely" for $\mathbf{V}$ is answered with "not likely at all" by the other three questions, and as a result, when preceded with the cue, the model rightly estimates that the chances of $\mathbf{V}$ being followed good is very low and as a result the model may correctly select $\mathbf{T}$ in those circumstances instead.

One could introduce into the model an attentional mechanism that weights the terms of the probe. The appropriate value of these weights could be gradually learned by the model as it discovers which relationships are reliable and informative predictors and which relationships are not reliable or not useful predictors. Such a mechanism has not been implemented in this model. 
Walsh and Anderson (2011) informed participants before the task "that the initial choice affected which cue appeared, and that the final choice depended only on the cue that appeared". Given this information, one might assume that participants only attended to two relationships:

1. The relationship between the initial choice, the cue shape that appears, and the feedback at the end of a trial, and

2. The relationship between the cue, the final choice, and the feedback received.

In our model, we instead make the following assumptions:

1. Participants may not have fully absorbed the clues provided by the instructions,

2. Attention and recall is, to a degree, automatic such that participants will pay attention to and be affected by things that they may know are irrelevant to the task, and that

3. The rapid pace of the experiment prevents participants from reasoning particularly carefully about the task and the decisions they are making.

Given these assumptions, we believe our model is a reasonable approximation to participant behaviour.

\subsection{Discussion}

The success of HDM as a model of the delayed-feedback learning task demonstrates that HDM can be applied to decision-making tasks that have been typically understood as utility learning tasks handled by a distinct procedural memory system. Indeed, converging evidence from the modeling literature suggests that procedural and declarative memory may not be so different (Humphreys, Bain, \& Pike, 1989a; Jamieson, Crump, \& Hannah, 2012; Jamieson \& Mewhort, 2011). 
While Walsh and Anderson (2011) have already modelled the task using temporal difference learning models that can be incorporated into the ACT-R production system, our model adopts an approach that is importantly different in two ways. First, as in Lebiere et al. (2007), decisions are made on the basis of generalizing from learned instances. Two, HDM is vector symbolic rather than symbolic.

Vector-symbolic memory systems can account for noise and generalization easily as part of the model's mechanisms and can efficiently and effectively model memory systems that contain very large quantities of data (Jones \& Mewhort, 2007). These advantages motivate us to extend the reach of HDM to decision-making.

In modelling human performance in this task, we addressed two novel questions for HDM/DSHM/BEAGLE models: (1) how to make the model sensitive to dependencies between non-consecutive events and (2) how to motivate the model to explore untried or infrequently tried choices and exploit choices associated with positive feedback. We accomplish the former by storing associations between non-consecutive events in memory. The latter we accomplish by initializing memory to a state of optimism, which encourages the model to explore.

There are some differences between the model's behaviour and the observed human behaviour, but our decision-making model is very simple, much simpler than what human participants are doing in the task. The model does not form, test, and reject hypotheses, nor is it curious, nor does it grow bored. Walsh and Anderson (2011) argue that the neural data from the experiment suggest a central role for surprise (or prediction error) in the task. Modelling surprise, attention, curiosity, or hypothesis testing, all provide routes forward for improving on this model. 


\section{Chapter 9 Memory for Abstract Associations}

How do humans learn the syntax and semantics of words from language experience? How does the mind discover abstract relationships between concepts? Computational models of distributional semantics (e.g., Landauer \& Dumais, 1997; Burgess \& Lund, 1997; Kwantes, 2005; Griffiths, Steyvers, \& Tenenbaum, 2007; Jones \& Mewhort, 2007) can analyze a corpus to derive representations of word meanings in terms of each word's relationship to all other words in the corpus. While these models are sensitive to topic (e.g., tiger and stripes) and synonymy (e.g., soar and fly), the models have limited sensitivity to part of speech (e.g., book and shirt are both nouns). By augmenting a holographic model of semantic memory (Jones \& Mewhort, 2007) with additional layers of representations, we demonstrate that sensitivity to syntax relies on exploiting higher-order associations between words. Our hierarchical holographic memory model bridges the gap between models of distributional semantics and unsupervised part-ofspeech induction algorithms (e.g., Christodoulopoulos, Goldwater, and Steedman, 2010), providing evidence that semantics and syntax exist on a continuum and emerge from a unitary cognitive system.

\subsection{Overview}

In what follows, we first define orders of association. Orders of association is a measure of the degree of separation between words or concepts, akin to the Erdős number (Goffman, 1969) or the Kevin Bacon (Fass, Ginelli, \& Turtle, 1996) number, but for human memory and language instead of films or research papers. We then briefly discuss the computational models of 
distributional semantics in the literature, before turning our attention to the BEAGLE model specifically (Jones \& Mewhort, 2007). We construct a Hierarchical Holographic Model by adding additional layers of representations to BEAGLE.

In Simulation 1, we run the Hierarchical Holographic Model on a simple artificial data set. We demonstrate that BEAGLE, corresponding to the first layer of the Hierarchical Holographic Model, is sensitive to second-order associations, but not third-order associations. Conversely, the Hierarchical Holographic Model is sensitive to third-order associations, and with the addition of more layers, can be made sensitive to associations of arbitrarily high order.

In Simulation 2, given sentences from a simple artificial grammar, we demonstrate that higher layers of the model develop increasingly well defined clusters corresponding to the syntactic categories of the grammar. In Simulation 3, with a more complex artificial data set, we demonstrate that higher layers of the model both allow the model to discover highly abstract associations and to suppress spurious similarities between concepts detected by the first layer (i.e., the BEAGLE model).

In Simulation 4 we run the hierarchical model on a collection of 15 children's novels. We find that higher layers of the model suppress semantically spurious similarities between words detected by the first layer (e.g., your and India). We find that, in particular, the higher layers vastly improve clustering for the syntactic category of determiners (e.g., she, there, my, your) as well as for some subtle syntactic/semantic categories such as directions (e.g., back, up, away). For example, the model does not discover that home is a direction until the fourth layer of the model. The higher layers also particularly reinforce the relationships between characters in the novels, verbs (e.g., get and have, had and were), and dialogue tags (e.g. said and answered). 
This work serves as both a preliminary demonstration of the importance of orders of association to language analysis as well as a proof of concept of Hierarchical Holographic Model.

\subsection{Orders of Association}

Saussure (1916) defines two types of relationships between words: paradigmatic and syntagmatic. A syntagmatic relationship is the syntactic relationship a word has with other words that surround it. A paradigmatic relationship is when a pair of words can be substituted for each other. Building on Saussure, we define the term order of association as a measure of the degree of separation of two words in an agent's language experience.

Measuring degrees of separation is the basis of the "Six Degrees of Kevin Bacon" parlour game (Fass, Ginelli, \& Turtle, 1996). In the game, players try to find the fewest number of steps away an actor is from having made a film with prolific film actor Kevin Bacon. The number of steps is referred to as an actor's Bacon number. Kevin Bacon has a Bacon number of zero. Anyone who has appeared in a film with Kevin Bacon has a Bacon number of one. For all other actors, if they have appeared in a film with an actor with a Bacon number of $n$, their Bacon number is $n+1$.

Less popular at parties but more popular at universities is the Erdös number (Goffman, 1969), the measure of how many steps away a researcher is from having co-authored a paper with prolific mathematician Paul Erdös. Measuring degrees of separation be applied not just to actors or researchers, but to words. Though when analyzing language, the frequency with which two words appear at given degrees of separation is more informative than the minimum distance. 
First order association is when two words appear together. In the sentence "eagles soar over trees", the words eagles and trees have first order association. Words with strong first order association (i.e., frequently appear close together) are often related in topic. For example, the words tiger and stripes have strong first order association.

Second order association is when two words appear with the same words. In the sentences "airplanes soar through skies" and "airplanes fly through skies", soar and fly have second order association. Words with strong second order association are often synonyms, or have a paradigmatic relationship in Saussure's terms.

Third order association is when two words appear with words that appear with the same words. Given the sentences in Table 9.1, the words eagles and birds have neither first nor second order association, but do have third order association.

Table 9.1. Artificial data set for Simulation 1.

\begin{tabular}{l}
\hline Sentences \\
\hline eagles soar over trees \\
birds fly above forest \\
airplanes soar through skies \\
airplanes fly through skies \\
airplanes glide through skies \\
dishes are over plates \\
dishes are above plates \\
dishes are atop plates \\
squirrels live in trees \\
squirrels live in forest \\
squirrels live in woods \\
\hline
\end{tabular}


One can keep abstracting to higher-level orders of association indefinitely. At sufficiently higher orders of association, all words are related to all other words. Sensitivity to increasingly higher-order associations allows one to identify increasingly abstract relationships between items, such as syntactic categories. We hypothesize that to properly capture the syntagmatic relationships between words in the English language, it is necessary for a cognitive model to be sensitive to at least third-order associations.

We can broaden our scope beyond language and define order of association as the degree of separation between any two items in an agent's life experience. Sensitivity to higher orders of association has applications to case-based reasoning. In case-based reasoning, agents use their knowledge of solutions to known problems to solve new problems. Successful case-based reasoning requires identifying potentially highly abstract commonalities between the current problem and previous, solved problems in order to select an appropriate approach to solving the problem.

For example, suppose you believe that a burglar has entered your house and you want something to defend yourself with. You know that a baseball bat would be a good weapon, but you look around and see that you don't have one. You do have a skateboard though, and it's enough like a baseball bat that you could use it instead. As easy as it is for people to make this substitution, identifying that skateboard is an appropriate substitute for a baseball bat requires a fairly abstract understanding of the relationship between the two items. Identifying such abstract relationships may require a cognitive model sensitive to higher-order associations. 


\subsection{Distributional Models of Semantics}

According to the philosopher Wittgenstein, "the meaning of a word is its use in language" (Wittgenstein, 1958, Sect. 43), or as the linguist Firth put it, "You shall know a word by the company it keeps" (Firth, 1957). Computational models of distributional semantics put this theory to the test by analyzing the relationships between words in a corpus to derive representations of each word's meaning.

Computational models of distributional semantics in the literature, such as LSA (Landauer \& Dumais, 1997), HAL (Burgess \& Lund, 1997), MINERVA 2 (Kwantes, 2005), the Topics Model (Griffiths, Steyvers, \& Tenenbaum, 2007), and BEAGLE (Jones \& Mewhort, 2007), are sensitive to only first and second-order associations. Jones and Mewhort observe clusters of vectors in semantic space that seem to correspond, roughly, to part of speech of information. Such clusters are suggestive of higher order associations, though BEAGLE does not exploit these higher order associations.

Kwantes (2005) demonstrates that the MINERVA 2 model of episodic memory (Hintzman, 1984) can be used to abstract a semantic representation from a term $\mathrm{x}$ document matrix. In a term $\mathrm{x}$ document matrix, each row is a term (or word), each column is a document in the corpus, and each entry in the matrix is the frequency with which the word appears in the given document. In the MINERVA 2 model, the process of memory retrieval produces an echo, a novel aggregate of individual experiences. When using a term $\mathrm{x}$ document matrix as the memories traces stored in a MINERVA 2 model, the echoes retrieved from memory are sensitive to higher orders of association and thus can serve as semantic representations (Kwantes, 2005).

According to Hintzman (1986), when we retrieve a memory it is always, to some degree, a generalization. A perception is stored in great detail, and on retrieval, we abstract away from 
detail to discover categories of perceptions based our history of perceptual experience. The MINERVA 2 model (Hintzman, 1984, 1986; Kwantes, 2005) demonstrates that the concepts and categories of a semantic memory system can be understood as emergent phenomena that arise from aggregating over the memory traces of individual episodes. These aggregates, or echoes, may themselves be, in turn, stored in memory, creating semantic memory traces. The question then arises, what are the effects of aggregating across echoes? What properties do the echoes of echoes have?

As we argue in Chapter 5, the semantic representations in the BEAGLE model of distributional semantics (Jones \& Mewhort, 2007) can be understood as the echoes that emerge from a MINERVA model. In other words, the units of BEAGLE are emergent from the units of MINERVA. We ask, what happens if we aggregate across the units of a BEAGLE model to create new units? And then aggregate across those units to create new units? And so on?

Inspired by Kwantes (2005), we have developed a model capable of detecting associations of arbitrarily high order. Using BEAGLE (Jones \& Mewhort, 2007; but see also Kelly, Kwok, \& West, 2015; Rutledge-Taylor, Kelly, West, \& Pyke, 2014, for variants on the model), a holographic model of semantic memory, as a basis, we present a hierarchical model that layers BEAGLE models on top of each other. The vector outputs of one layer serve as the vector inputs to the next layer. The model roughly resembles a deep neural network in structure, but unlike a neural network, the model does not use stochastic gradient descent and the data is not subject to dimensional reduction by higher layers of the model. 


\subsection{The BEAGLE Model}

In Jones and Mewhort's (2007) BEAGLE model of semantic memory, each word is represented by two vectors: an environment vector that represents the percept of a word and a memory vector that represents the concept of a word.

An environment vector (denoted by e) stands for what a word looks like in writing or sounds like when spoken. In our simulations, environment vectors are randomly generated by sampling values from a Gaussian distribution with a mean of zero and a variance of $1 / n$, where $n$ is the dimensionality of the vectors. Alternatively, instead of using random numbers, environment vectors can be generated to accurately represent the phonemes or graphemes that compose the words, as in Cox, Kachergis, Recchia, and Jones (2011). Incorporating the phonemes or graphemes has the potential to improve performance by allowing the model to be sensitive to a word's morphology (e.g., cat and cats or skate and skating have shared morphology indicating closely related meaning). Unsupervised part of speech tagging algorithms that incorporate morphology tend to out-perform those that do not (Christodoulopoulos, Goldwater, and Steedman, 2010).

Memory vectors (denoted by $\mathbf{m}$ ) represent the associations a word has with other words. Memory vectors are constructed as the model reads the corpus. The memory vectors are holographic in that they use circular convolution (denoted by *) to compactly encode associations between words (Plate, 1995). Given a sentence, for each word in the sentence, vectors representing all sequences of words in the sentence (or grams) that include the target word are summed together and added to the target word's memory vector. 
For example, given the sentence, "eagles soar over trees", we update the memory vectors for each word in the sentence: eagles, soar, over, and trees. Each memory vector is updated with a sum of grams. For example, the memory vector for the word soar, $\mathbf{m}_{\text {soar }}$, is updated with the bigrams "eagles soar" and "soar over", the trigrams "eagles soar over" and "soar over trees", and the tetragram "eagles soar over trees".

Each gram is constructed as a convolution of the environment vectors of the constituent words, except for the target word, which is represented by the placeholder vector (denoted by Ф). The placeholder vector is randomly generated and serves as a universal retrieval cue. With the placeholder substituted for the target word, each gram can be understood as a question to which the target word is the answer. So, rather than adding a representation of "eagles soar over" in $\mathbf{m}_{\text {soar, }}$ we instead add "eagles ? over", i.e., "What was the word that appeared between eagles and over?". Each memory vector can be understood as the sum of all questions to which that memory vector's word is an appropriate answer.

For example, given "eagles soar over trees", we update $\mathbf{m}_{\text {soar }}$ as follows:

$$
\begin{aligned}
\mathbf{m}_{\text {soar, t+1 }}=\mathbf{m}_{\text {soar, }}+\mathbf{P}_{\text {before }}\left(\mathbf{e}_{\text {eagles }}\right) * \boldsymbol{\Phi}+\mathbf{P}_{\text {before }}(\boldsymbol{\Phi}) * \mathbf{e}_{\text {over }}+ \\
\mathbf{P}_{\text {before }}\left(\mathbf{P}_{\text {before }}\left(\mathbf{e}_{\text {eagles }}\right) * \boldsymbol{\Phi}\right) * \mathbf{e}_{\text {over }}+\mathbf{P}_{\text {before }}\left(\mathbf{P}_{\text {before }}(\boldsymbol{\Phi}) * \mathbf{e}_{\text {over }}\right) * \mathbf{e}_{\text {trees }}+ \\
\mathbf{P}_{\text {before }}\left(\mathbf{P}_{\text {before }}\left(\mathbf{P}_{\text {before }}\left(\mathbf{e}_{\text {eagles }}\right) * \boldsymbol{\Phi}\right) * \mathbf{e}_{\text {over }}\right) * \mathbf{e}_{\text {trees }}
\end{aligned}
$$

i.e., to $\mathbf{m}_{\text {soar }}$ we add "eagles ?", "? over", "eagles ? over", "? over trees", and "eagles ? over trees", where $t$ is the current time step, and $\mathbf{P}_{\text {before }}$ is a randomly generated permutation matrix used to indicate that a word occurred earlier in the sequence. 


\subsection{Hierarchical Holographic Model}

The hierarchical holographic model is a series of BEAGLE models, such that the memory vectors of one model serves as the environment vectors for the next model. Layer 1 is a standard BEAGLE model with randomly generated environment vectors. Once Layer 1 has been run on a corpus, Layer 2 is initialized with Layer 1's memory vectors as its environment vectors. Layer 2 is run on the corpus to generate a new set of memory vectors, which in turn are used as the environment vectors for the next layer, and so on, for as many layers as desired.

To use the memory vectors of a previous layer as the memory vectors for the next layer, it is necessary to normalize and randomly permute the vectors (Kelly, Blostein, and Mewhort, 2013). For layer $l+1$, and all words $i$, the environment vectors for that layer are:

$$
\mathbf{e}_{i, l+1}=\mathbf{P}_{\text {group }}\left(\frac{\mathbf{m}_{i, l}}{\sqrt{\mathbf{m}_{i, l} \bullet \mathbf{m}_{i, l}}}\right)
$$

where $\mathbf{P}_{\text {group }}$ is a random permutation used to transform memory vectors into environment vectors and $\bullet$ is the dot product.

Running each layer of the model on the corpus sequentially, such that a higher layer is run only once the lower layer is finished, is cognitively implausible but computationally desirable. For the sake of cognitive plausibility, the layers could instead be run in parallel, such that a higher layer uses as perceptual vectors permuted and normalized versions of what the lower layer memory vectors are at any particular moment. Running the layers in parallel would make the higher layer vectors noisier as the lower layer memory vectors used to construct them are in a state of flux. Running layers in parallel would increase the amount of experiences necessary to construct good representations at the higher layers. Thus, for the sake of providing a 
clear demonstration of what the model can do, we have decided to run the layers of the model sequentially rather than in parallel.

To demonstrate how the hierarchical holographic model behaves, we have run three simulations. In Simulation 1, using a small artificial data set, we demonstrate that the model can detect third-order associations. In Simulation 2, we demonstrate that the model can detect the syntactic categories of a simple artificial grammar. In Simulation 4, we run the model on an English language corpus and demonstrate that the model exploits higher-order associations at higher layers to refine its representations of the relationships between words.

\subsection{Simulation 1: Results}

Higher layers of the model are sensitive to higher orders of association (Figure 9.1), as demonstrated by an artificial data set (Table 9.1). The memory vectors for words with second order association, such as soar and fly, are close on Layer 1 (see Table 9.2, cosine $=0.44$ ) and draw closer in higher layers (Layer 4 , cosine $=0.71$ ). Whereas eagle and bird, which have only third order association, are distant on Layer 1 (see Figure 9.1, cosine $=-0.08$ ) but are closer on Layer $2(\operatorname{cosine}=0.16)$ and draw closer still in higher layers (Layer 4 , cosine $=0.47)$. Figure 9.1 shows cosine distances between 256 dimensional vectors, the distances compressed to 3 dimensions by multi-dimensional scaling. 

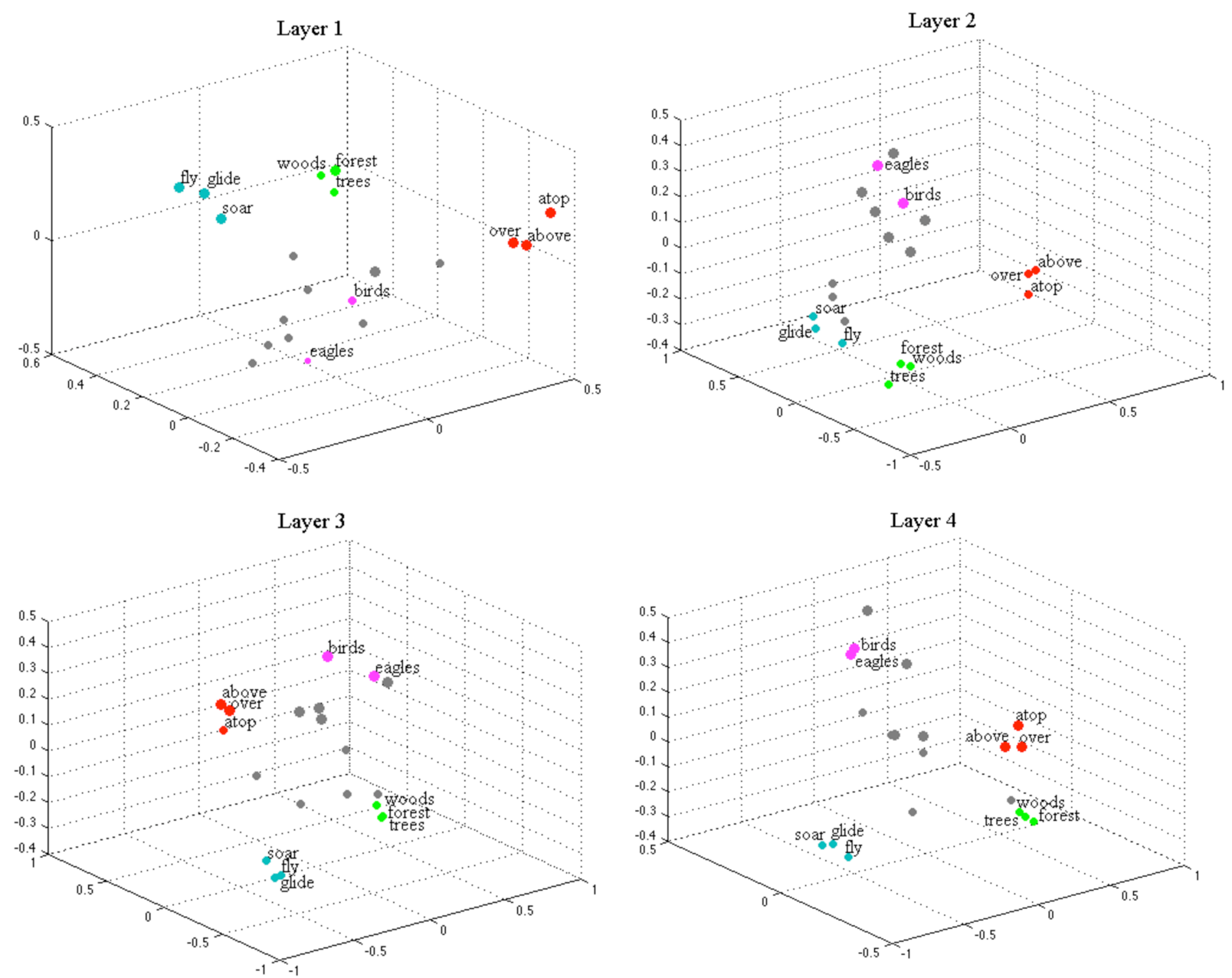

Figure 9.1. Cosine distances between memory vectors for Simulation 1, Layers 1 to 4, compressed to 3 dimensions using multi-dimensional scaling.

Table 9.2. Cosine similarities between pairs of memory vectors in Simulation 1.

\begin{tabular}{lllll}
\hline Layer & $\operatorname{cosine}\left(\mathbf{m}_{\text {eagles }}, \mathbf{m}_{\text {birds }}\right)$ & $\operatorname{cosine}\left(\mathbf{m}_{\text {soar }}, \mathbf{m}_{\text {fly }}\right)$ & $\operatorname{cosine}\left(\mathbf{m}_{\text {over }}, \mathbf{m}_{\text {above }}\right)$ & $\operatorname{cosine}\left(\mathbf{m}_{\text {trees }}, \mathbf{m}_{\text {forest }}\right)$ \\
\hline $\mathbf{1}$ & -0.08 & 0.44 & 0.46 & 0.41 \\
$\mathbf{2}$ & 0.16 & 0.55 & 0.56 & 0.56 \\
$\mathbf{3}$ & 0.38 & 0.56 & 0.69 & 0.61 \\
$\mathbf{4}$ & 0.47 & 0.71 & 0.68 & 0.74 \\
\hline
\end{tabular}




\subsection{Simulation 1: Discussion}

Simulation 1 provides a simple example of the effect of the higher layers. Each memory vector at Layer 1 is constructed as a sum of convolutions of environment vectors. As such, the memory vectors at Layer 1 encode first order associations with respect to the environment vectors, measuring the frequency with which each word co-occurs with other words and sequences of words. The cosines between memory vectors are a measure of second-order association, the degree to which the two words co-occur with the same words. The algorithm that produces Layer 1 transforms data that captures first-order association (co-occurrence) into data that captures second-order associations. The algorithm is a step, and by repeating that algorithm to produce higher layers, we can build a staircase.

Layer 1 of the model has no ability to detect third-order associations. A pair of words with third-order association, but not first or second, do not appear together in the same sentence and do not co-occur with the same words. As such, the memory vectors for a pair of words with only third-order association will be constructed from disjoint sets of environment vectors. In

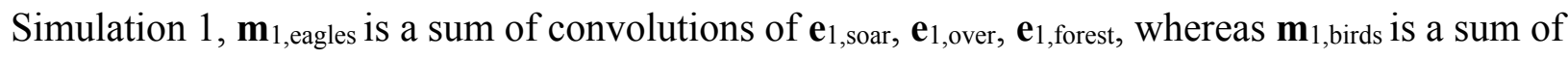
convolutions of $\mathbf{e}_{1, f \mathrm{fy}}, \mathbf{e}_{1, \text { above }}, \mathbf{e}_{1, \text { trees. }}$. As the environment vectors are approximately orthogonal, the memory vectors constructed from them will also be approximately orthogonal. As a result, $\mathbf{m}_{1, \text { eagles }}$ and $\mathbf{m}_{1, \text { birds }}$ are approximately orthogonal on Layer $1(\operatorname{cosine}=-0.08)$.

But at higher layers of the model, the environment vectors are no longer orthogonal. The environment vectors for Layer 2 are the memory vectors for Layer 1 . As a result, $\mathbf{e}_{2, \text { soar }}$ is similar

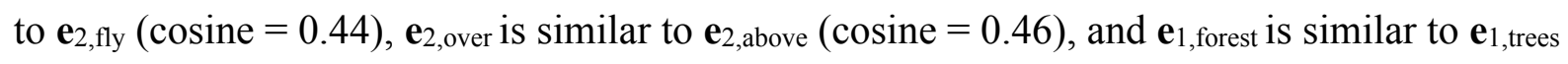
(cosine $=0.41$; see Table 9.2). Even though $\mathbf{m}_{2, \text { birds }}$ and $\mathbf{m}_{2, \text { eagles }}$ are still constructed from disjoint 
sets of environment vectors, because the vectors that the memory vectors are constructed from are similar, the memory vectors themselves are somewhat similar $(\operatorname{cosine}=0.16)$. As the Layer 2 memory vectors for the pairs soar and fly, above and over, and forest and trees are more similar at Layer 2 than at Layer 1 (see Table 9.2), the environment vectors for them at Layer 3 will be more similar, which will further drive up the similarity between eagles and birds, such that by Layer 4, the memory vectors for eagles and birds have strong similarity (cosine $=0.47$ ).

\subsection{Simulation 2}

The purpose of Simulation 2 is to provide a clean demonstration of the hierarchical holographic model's ability to detect syntactic categories. We use a simple artificial grammar, where all sentences are sequences of the form $A B C D$, where $A, B, C$, and $D$ are categories, each with 5 possible values (e.g., $A=\left\{a_{1}, a_{2}, a_{3}, a_{4}, a_{5}\right\}$ ). Given all 625 possible sentences in this grammar, all words have first-order association with words belonging to a different category (e.g., $a_{3}$ and $c_{1}$ ) and second-order association with all words belonging to the same category (e.g., $a_{3}$ and $a_{1}$ ). However, instead of using all 625 sentences, we gave the model a random subset of 15 sentences.

Figure 9.2 shows a run of the model on a random 15 sentences generated by the grammar. As in Figure 9.1, Figure 9.2 shows cosine distances between 256 dimensional vectors, the distances compressed to 3 dimensions by multi-dimensional scaling. In the set of 15 sentences for Figure 9.2, 7\% of word pairs had third-order associations, but no first or second-order associations (i.e., 7\% of words never appeared together in a sentence and never appeared in sentences that shared any words with sentences that the other word appeared in). In Figure 9.2, we see that with higher layers of the model, the four categories form increasingly distinct 
clusters. At Layer 1, the mean within category cosine is 0.18 with a standard deviation of 0.14 , and between categories the mean cosine is 0.00 with a standard deviation of 0.06 . At Layer 4 , the mean within category cosine is 0.61 with a standard deviation of 0.18 , and between categories the mean cosine is 0.04 with a standard deviation of 0.08 .
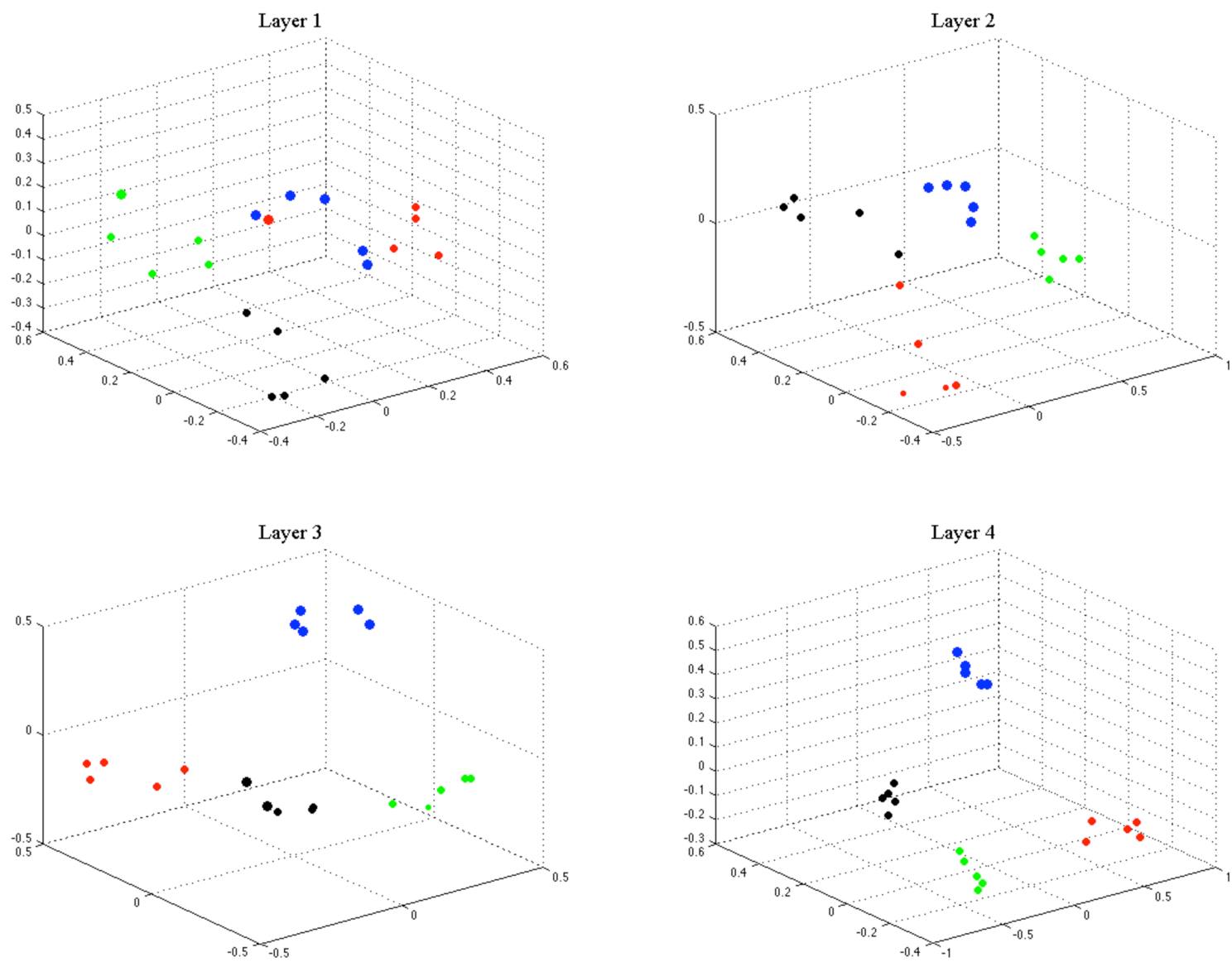

Figure 9.2. Cosine distances between memory vectors for Simulation 2, Layers 1 to 4, compressed to 3 dimensions using multi-dimensional scaling.

\subsection{Simulation 3: Method}

The purpose of Simulation 3 is two-fold. First, we intend to demonstrate how the model performs on an artificial data set that is more complex than in Simulations 1 and 2, but still 
simpler than natural language, allowing us to analyze its performance more easily than in Simulation 4.

Second, we wish to suggest uses of the model beyond language. The hierarchical holographic model is not a model of language cognition per se, but rather a model of memory organized around concepts. While these concepts may be referred to by words, these concepts are not necessarily words. DSHM (Rutledge-Taylor et al., 2014) and HDM (Kelly, Kwok, \& West, 2015) extend the BEAGLE model to non-linguistic tasks, such as the fan-effect memory task, the problem size effect in math cognition, and learning iterated decision-making tasks such as rock-paper-scissors. The hierarchical holographic model is an extension of BEAGLE to capture higher orders of association between concepts, and likewise can be extended to nonlinguistic tasks.

For Simulation 3 we imagine a world populated by strange animals with strange habits. The model represents what an agent knows about the world, and we explore what inferences about commonalities the agent is able to make at higher layers of the model. Perhaps our agent is a hapless adventurer in a foreign and magical land with only the scribblings in a cryptic bestiary to guide them.

The artificial data set for Simulation 3 is still language-like in form and is, in its essential structure, very similar to Simulation 2 , though with a more complex and difficult grammar. The data set consists of 100 randomly generated facts about animals. There are 20 animals and 5 facts about each animal. Each animal is identified by a region, colouring and a type. Each fact is of the form "[region] [colouring] [type] [relation] [object]", e.g., the "eastern green cerastes spits 
venom" or the "northern pinstriped jaculus eats leucrota" (see Table 9.3). The names of the animal types are drawn from medieval bestiaries.

Table 9.3. Artificial data set for Simulation 1.

\begin{tabular}{|c|c|}
\hline Western Grey Catoblepas & Western Tusked Amphisbaena \\
\hline $\begin{array}{l}\text { - has wings } \\
\text { - has four legs } \\
\text { - spits fire } \\
\text { - spits goo } \\
\text { - is related to the monocerus }\end{array}$ & $\begin{array}{l}\text { - makes honey } \\
\text { - makes songs } \\
\text { - builds nests } \\
\text { - avoids the yale } \\
\text { - is related to the jaculus }\end{array}$ \\
\hline
\end{tabular}

\subsection{Simulation 3: Results}

Figure 9.3 plots cosine distances between concepts in Simulation 3, concepts represented by 256 dimensional vectors and distances compressed to two dimensions. Colours indicate concept category (animal type, region, colouring, habitat, anatomical features, etc.). Clustering for the categories is weaker than in Simulation 2, but the clustering does improve across layers.

At Layer 1 , the within category mean cosine is 0.09 with a standard deviation of 0.15 , and the between categories mean cosine is 0.00 with a standard deviation of 0.07 . By Layer 4 , in Figure 9.3, we can see that type of animal occupies the lower-left corner of the plot, animal products (e.g., milk, honey, wool) occupy the upper-left, anatomical features (e.g., wings, eyes, fins) occupy the upper-right, and colourings and relations occupy the lower-right. By Layer 4, we find a corresponding increase in within-category cosine $($ mean $=0.18$, standard deviation $=0.21)$ whereas between-category cosine remains low $($ mean $=0.02$, standard deviation $=0.08)$. 

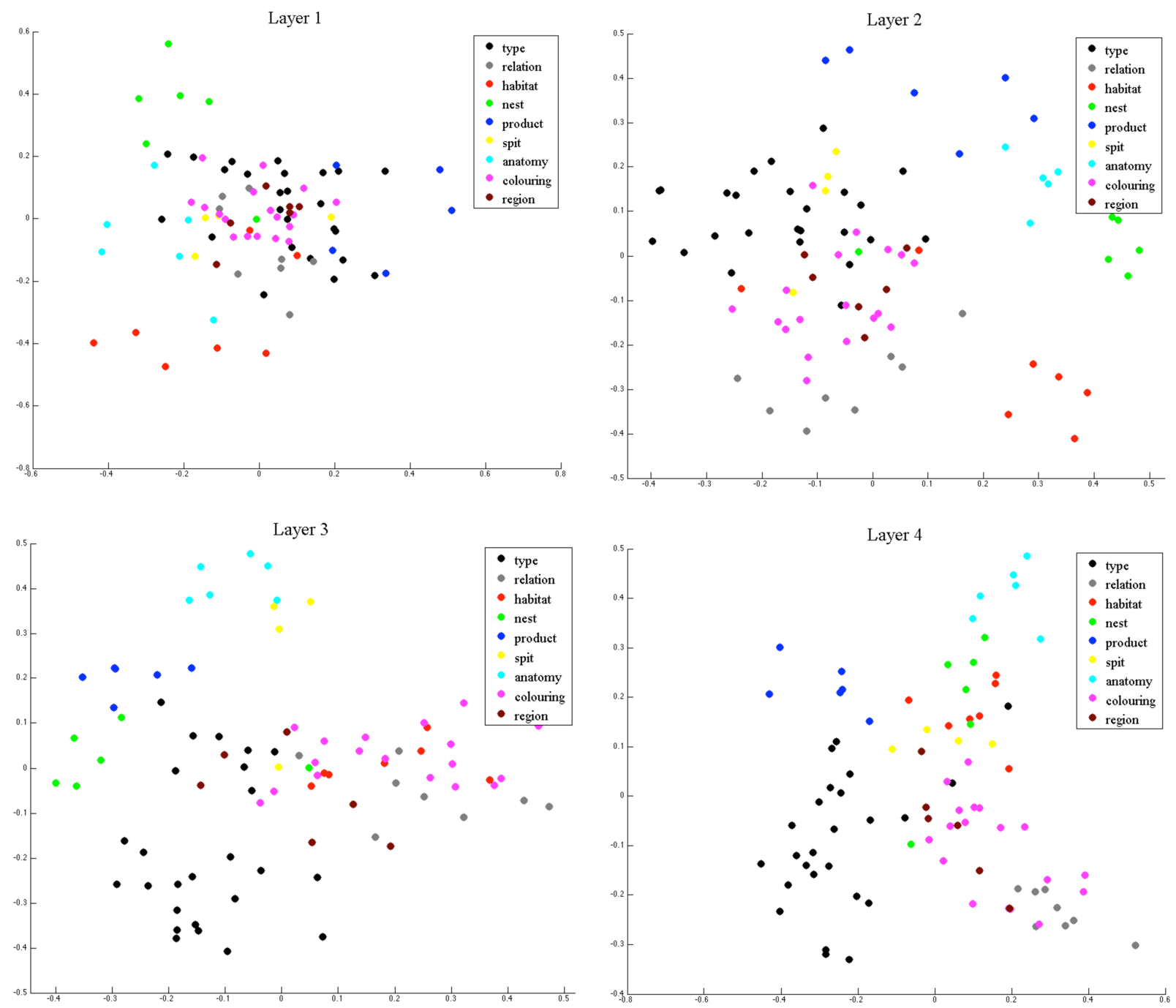

Figure 9.3. Cosine distances between memory vectors for Simulation 3, Layers 1 to 4, compressed to 2 dimensions using multi-dimensional scaling.

Is the model usefully exploiting the higher-order associations in the Simulation 3 data set? We selected pairs of concepts from the 100 pairs of concepts with the highest cosines on Layer 1 (Figure 9.4) or Layer 4 (Figure 9.5). Open marker and solid line indicates the five pairs that increased in similarity the most from Layer 1 to Layer 4. Filled marker and dotted line indicates the five pairs that increased in similarity the least from Layer 1 to Layer 4. 


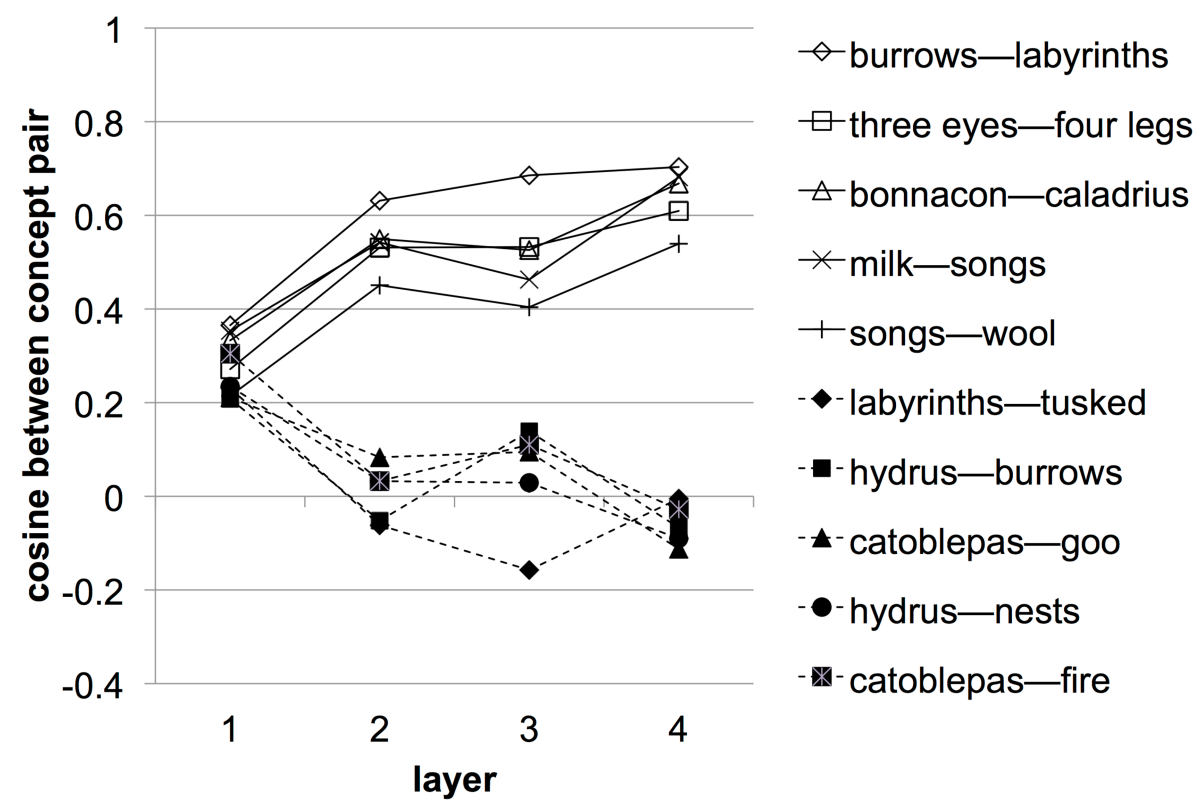

Figure 9.4. Cosine similarities between memory vector pairs across layers for Simulation 3. Pairs are selected from the top 100 most similar pairs on Layer 1. Open marker and solid line indicates the 5 pairs that increased in similarity the most from Layer 1 to Layer 4 . Filled marker and dotted line indicates the 5 pairs that increased in similarity the least from Layer 1 to Layer 4.

In Figure 9.4, we see that similarity between within-category pairs is strengthened by higher layers of the model. Similarity is increased between animal products (milk, song, and wool), animal nests (labyrinths and burrows), anatomical features (eyes and legs), and the animals themselves (bonnacon and caladrius). Conversely, the cosines of between-category pairs that were among the top 100 at Layer 1 are suppressed at higher layers.

In Figure 9.5, we see that of the top 100 pairs on Layer 4, the five pairs that increased the least from Layer 1 to Layer 4 are within-category pairs that already had strong similarity at Layer 1 (goo and fire are both things that the animals spit, and gems and songs are both animal products). This demonstrates that strong relationships correctly discovered by Layer 1 are maintained at higher layers. 


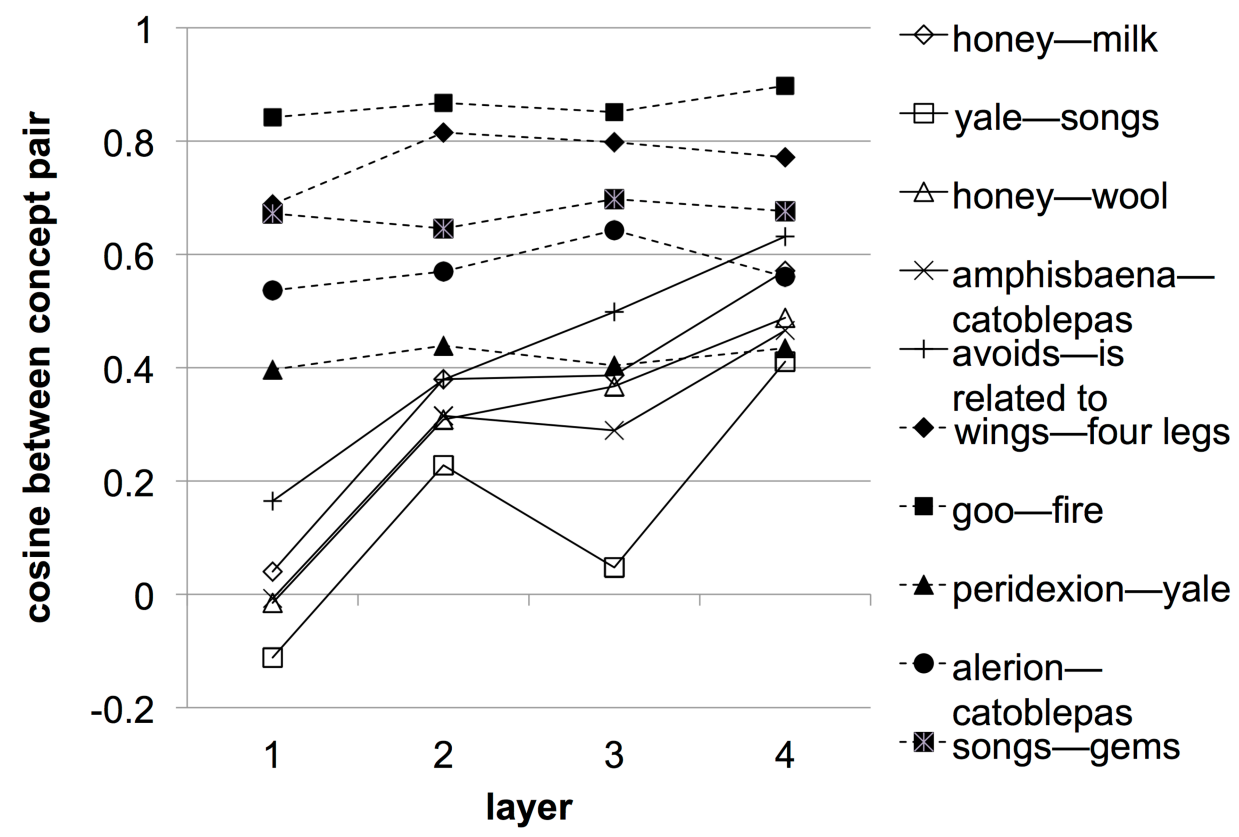

Figure 9.5. Cosine similarities between memory vector pairs across layers for Simulation 3. Pairs are selected from the top 100 most similar pairs on Layer 1. Open marker and solid line indicates the 5 pairs that increased in similarity the most from Layer 1 to Layer 4. Filled marker and dotted line indicates the 5 pairs that increased in similarity the least from Layer 1 to Layer 4.

In Figure 9.5, the five pairs that increased the most from Layer 1 to Layer 4 demonstrate that higher layers are discovering relationships between concepts that are entirely absent at Layer 1. Four out of five are within-category pairs: honey, milk, and wool are all animal products and amphisbaena and catoblepas are both animal types. The between category pair yale and songs is erroneous, but a result of the structure of the randomly generated data set. The yale appears only in two facts as an object to be avoided. No facts have the yale as a subject. That yale and songs develop a relationship at higher layers of the model is likely due to the fact that they are both objects of animal behaviour, specifically the behaviours avoiding the yale and making songs. This is an abstract, syntactic association. The pair perixion and yale are also present in Figure 9.5 
and have a more or less constant similarity strength across the layers. Like the yale, the perixion only appears in two facts as an object to be avoided.
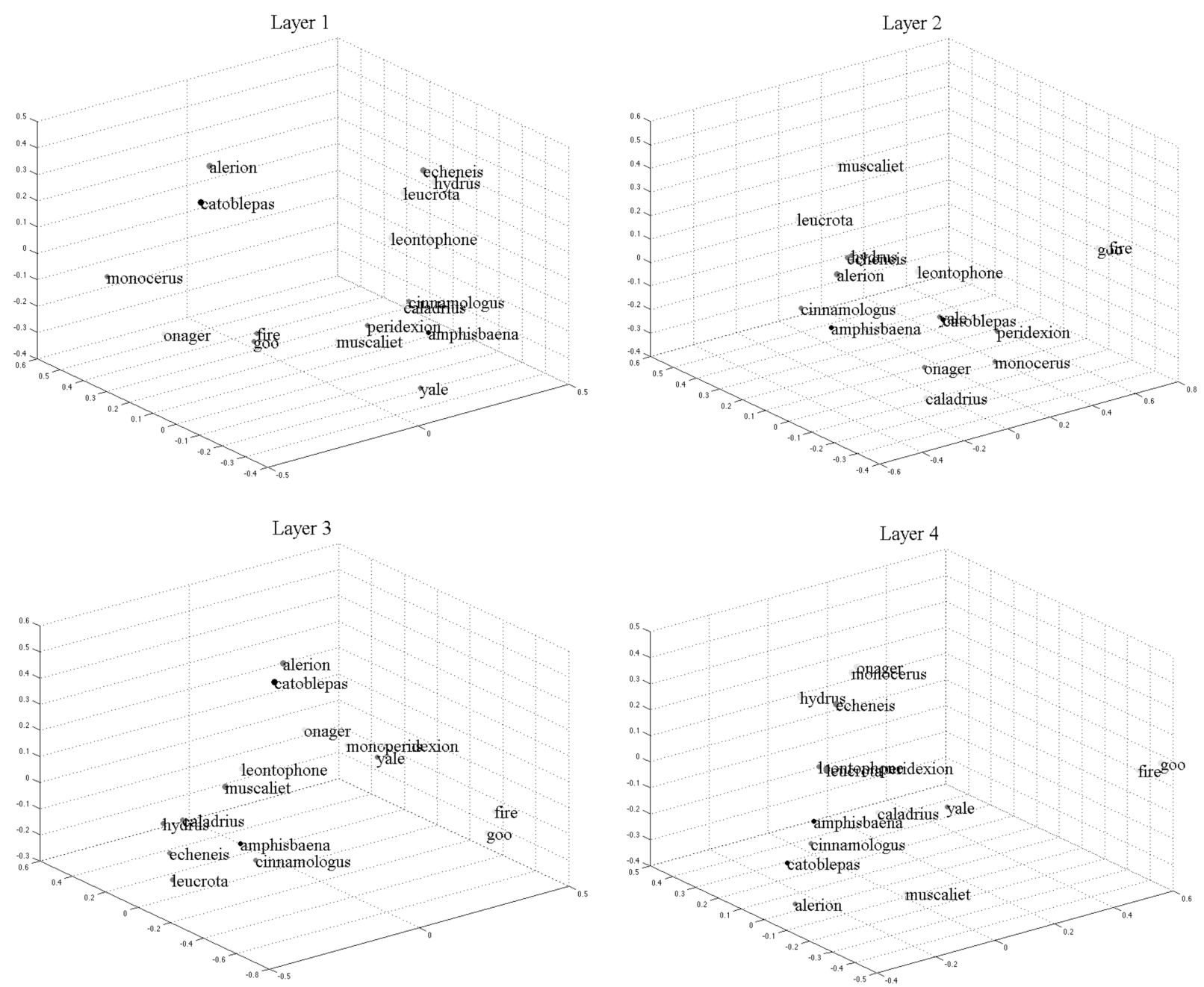

Figure 9.6. Cosine distances for target animals amphisbaena and catoblepas and their five nearest neighbours at each layer. Markers indicate target (black), neighbour (grey), and not a neighbour (light grey). Compressed to 3 dimensions using multi-dimensional scaling.

In Figure 9.6, we examine the amphisbaena and catoblepas pair from Figure 9.5 in more detail, plotting them alongside their five nearest neighbours at each layer of the model. At Layer 1 , the amphisbaena and catoblepas are wholly dissimilar (cosine $=-0.01)$, as we would expect 
given the very different set of facts the model knows about the two animals (see Table 9.3). The amphisbaena and the catoblepas are both related to other animals, the monocerus and the jaculus respectively, but otherwise the facts about the two animals have nothing in common. By Layer 4, the model has discovered that the amphisbaena and the catoblepas are both animals, clustering them together in space with most of the other animals. At Layer 1, the catoblepas, which spits fire and goo (Table 9.3), is near-neighbours to fire and goo. By Layer 4, fire and goo have been pushed away in the space and are no long near neighbours to any of the animals (except for the yale, which is something of a special case).

\subsection{Simulation 3: Discussion}

The results of Simulation 3 suggest that higher layers of the model generally strengthen withincategory cosines while suppressing between-category cosines (see Figure 9.4) as a result of progressive reorganization of the space into categorial clusters as they are discovered by the model (see Figures 9.3 and 9.6). Additionally, higher layers of the model are able to discover new relationships in the data absent at Layer 1 (see Figure 9.5).

\subsection{Simulation 4}

We ran the model on 15 out-of-copyright books from the The Bobbsey Twins series of children's novels, available through Project Gutenberg. The corpus consists of 441476 words and 9062 unique words occurring in 34399 sentences with a maximum sentence length of 81 words. The model was run using 256 dimensional vectors and four layers. The model read the corpus one sentence at a time. Within each sentence, the model used a moving window of 21 words, 10 
words to the left and right of a target word. Within that window, all grams, from bigrams up to 21-grams the width of the window, are encoded as convolutions of environment vectors and summed into the target word's memory vector.

Table 9.4. Top 10 word-pairs with the highest cosines on each layer in Simulation 4.

\begin{tabular}{lllllrll}
\hline Layer 1 & \multicolumn{3}{c}{ Layer 2 } & \multicolumn{3}{c}{ Layer 3 } & \multicolumn{2}{c}{ Layer 4 } & \\
\hline Mrs. Mr. & 0.94 & Mrs. Mr. & 0.96 & he she & 0.99 & he she & 1.00 \\
laugh smile & 0.88 & he she & 0.95 & Nan Bert & 0.98 & Nan Bert & 0.99 \\
in into & 0.85 & Nan Bert & 0.94 & on in & 0.98 & on in & 0.99 \\
in from & 0.84 & can can't & 0.93 & asked said & 0.98 & he they & 0.99 \\
on in & 0.83 & asked said & 0.93 & Mrs. Mr. & 0.98 & Freddie Nan & 0.99 \\
guess hope & 0.83 & asked cried & 0.92 & exclaimed cried 0.97 & Nan Harry & 0.99 \\
'll can & 0.82 & 'll can & 0.92 & in from & 0.97 & Freddie Harry & 0.99 \\
in of & 0.82 & 'll can't & 0.92 & Freddie Bert & 0.97 & my your & 0.99 \\
on from & 0.80 & don't didn't & 0.92 & he they & 0.97 & in at & 0.99 \\
wish guess & 0.80 & exclaimed cried 0.91 & is was & 0.97 & on at & 0.99 \\
\hline
\end{tabular}

For the purposes of our analysis, at each layer of the model we looked at the 100 word pairs with the highest cosines. We find that all layers of the model produce intuitive results (see Table 9.4 for the top 10 word pairs on each layer). The cosines at higher layers have larger means and larger standard deviations (Table 9.5), as we would expect with tighter clustering of words at higher layers as seen in Simulation 2.

We find that higher layers of the model exploit higher-order associations to strengthen semantically and syntactically correct relationships only weakly present in Layer 1 (see Figure 9.7) and to suppress erroneously strong relationships present in Layer 1 (see Figure 9, the relationship of Chicago and Meadowbrook to our). 
Table 9.5. Cosine means and standard deviations for each layer in Simulation 4.

\begin{tabular}{lll}
\hline Layer & mean cosine & standard deviation \\
\hline $\mathbf{1}$ & 0.01 & 0.07 \\
$\mathbf{2}$ & 0.03 & 0.08 \\
$\mathbf{3}$ & 0.09 & 0.12 \\
$\mathbf{4}$ & 0.18 & 0.17 \\
\hline
\end{tabular}

In Figure 9.7, we examine the effect of the four layers of the model. Open marker and solid line indicates the 5 word pairs that increased in similarity the most from Layer 1 to Layer 4, word pairs selected from the top 100 word pairs on Layer 4. Filled marker and dotted line indicates the 5 word pairs that increased in similarity the least from Layer 1 to Layer 4, word pairs selected from the top 100 word pairs on Layer 1.
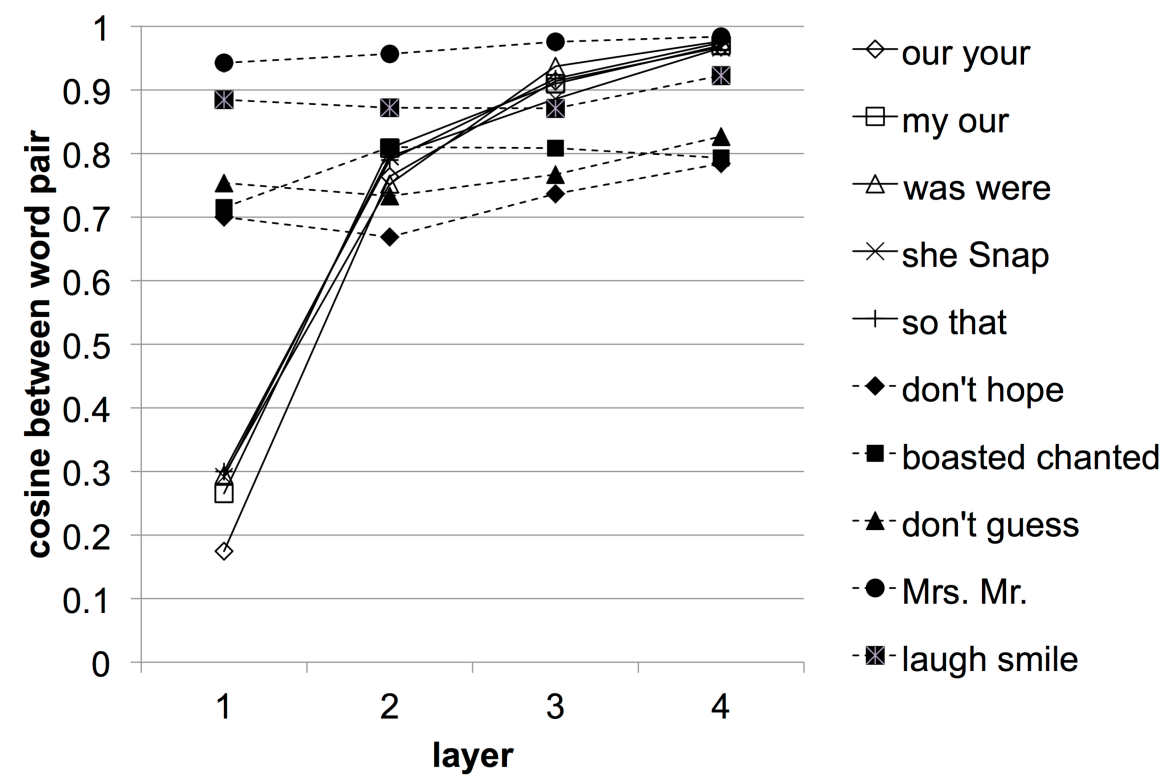

Figure 9.7. Cosine similarities between word pairs across layers in Simulation 4. Open marker and solid line indicates the 5 pairs that increased in similarity the most from Layer 1 to Layer 4, selected from the top 100 most similar pairs on Layer 4. Filled marker and dotted line indicates the 5 pairs that increased in similarity the least from Layer 1 to Layer 4, selected from the top 100 most similar pairs on Layer 1. 
From the contrast between the open marker and filled marker word pairs in Figure 9.7 we can see that the higher layers differentially effect word pairs. The filled marker word pairs remain relatively constant across the layers whereas we see a dramatic increase in similarity for the open marker pairs. For example, the possessive determiners our and your increase from a cosine of 0.17 at Layer 1 to 0.97 at Layer 4. Conversely, word pairs such as the verbs laugh and smile $($ Layer 1 cosine $=0.88$, Layer 4 cosine $=0.92)$ or the titles Mr. and Mrs. $($ Layer 1 cosine $=$ 0.94, Layer 4 cosine $=0.98$ ) are already highly similar at Layer 1 and so increase little across layers. We can see that the fact that the filled marker word pairs remain mostly constant across the layers is not merely due to a ceiling effect. For example, the pair of verbs don't and hope, moderately similar at Layer $1(\operatorname{cosine}=0.70)$, do not increase at Layer $2(\operatorname{cosine}=0.67)$ and only moderately increase at higher layers (Layer 4 , cosine $=0.78$ ).

The word pair she and Snap merits explanation. Snap is the name of the Bobbsey Twins' pet dog. Snap is male. Because Snap is male, Snap has a stronger relationship to he (cosine $=$ $0.37)$ at Layer 1 than she $(\operatorname{cosine}=0.30)$, though neither relationship is particularly strong. By Layer 2, the model discovers proper names can be substituted for pronouns and Snap now has a strong relationship to both he and she (cosine $=0.80$ for both). At Layer 1 , he and she are strongly related $(\operatorname{cosine}=0.74)$, but by Layer 2 the two pronouns become close to identical $(\operatorname{cosine}=0.95)$ and by Layer 4 they are indistinguishable $(\operatorname{cosine}=1.00)$. Thus, as he and she are nearly identical at higher layers, Snap is necessarily closely related to both pronouns at higher layers. However, because Snap is more closely related to he at Layer 1, the relationship between 
she and Snap increases more across the layers, which is why it is among the 5 word pairs that increase the most from Layer 1 to Layer 4 (as selected from the top 100 word pairs at Layer 4).

From Figure 9.7 we can also see that the higher layers of the model seem to have a diminishing ability to detect additional, useful semantic or syntactic information. For the five word pairs that increase the most across the layers (open markers), we see a dramatic increase from Layers 1 to 2, a modest increase from Layers 2 to 3, and a slight increase to Layer 4.

That higher layers provide a diminishing advantage is supported by Figure 9.8, in which we examine the effect of each layer. For Layers 2 to 4 , we select the 5 word pairs from the top 100 of that layer that increased the most from the previous layer. For contrast, we also select the 5 word pairs from the top 100 of the previous layer that decreased the most by the target layer.

In Figure 9.8 (i) we see a dramatic increase for the open-marker word pairs (the determiners his, my, your, her, their, and the verbs could might and will) from Layer 1 to Layer 2, which provides evidence that the model is indeed exploiting third order associations to gain additional word knowledge. For the closed marker word pairs in Figure 9.8 (i), the ones that change the least from Layer 1 to 2, we see that some of them are slightly inhibited by the Layer 2 associations, before increasing at higher layers. The increase of the closed marker at higher layers (e.g., the increase of bit and few at from Layer 3 to Layer 4) suggest that associations higher than three are being exploited. However, the (open marker) word pairs that increase the most from Layer 2 to 3 or Layer 3 to 4 in Figures 9.8 (ii) and (iii) (e.g., Snap and Snoop, the Bobbsey Twins' pet dog and cat) are all word pairs that increased significantly from Layer 1 to 2, suggesting that for the most part, Layers 3 and 4 are merely reinforcing word pairs already discovered at Layers 1 and 2. 


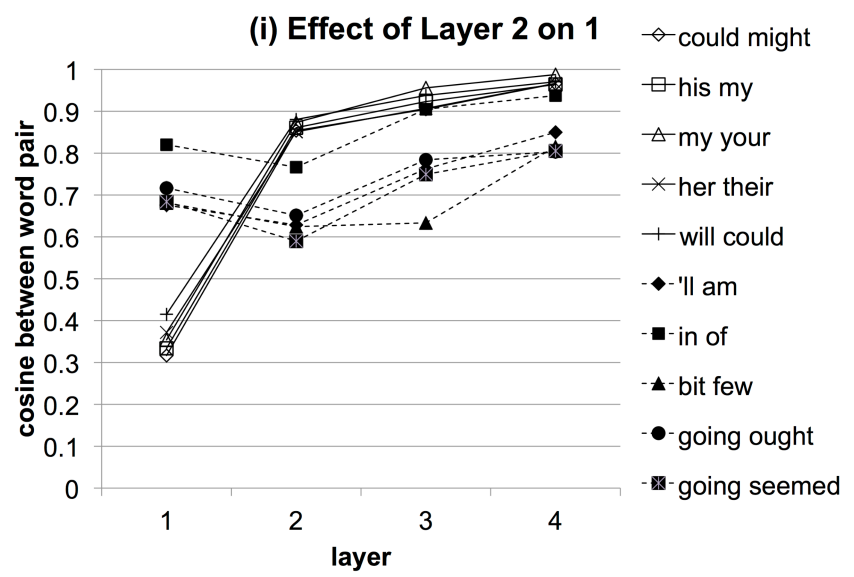

(ii) Effect of Layer 3 on $2 \rightarrow$ with at

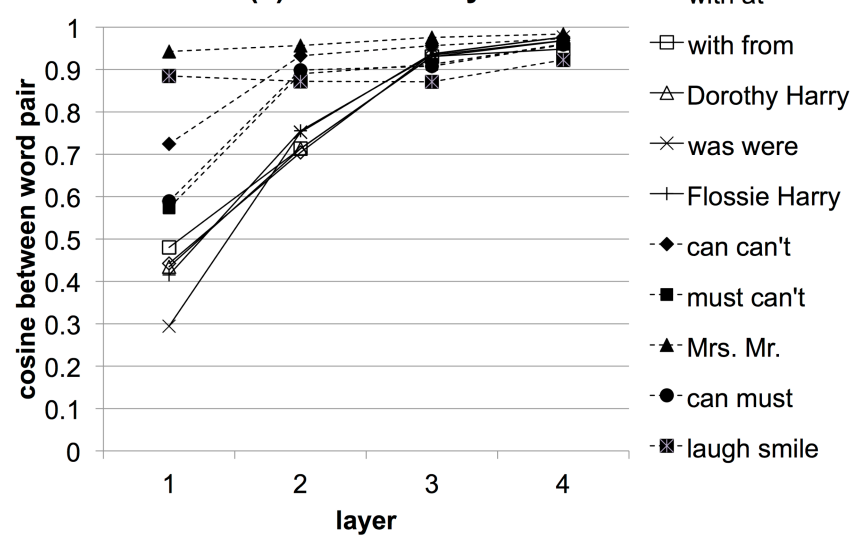

(iii) Effect of Layer 4 on $3 \diamond$ Snap Snoop

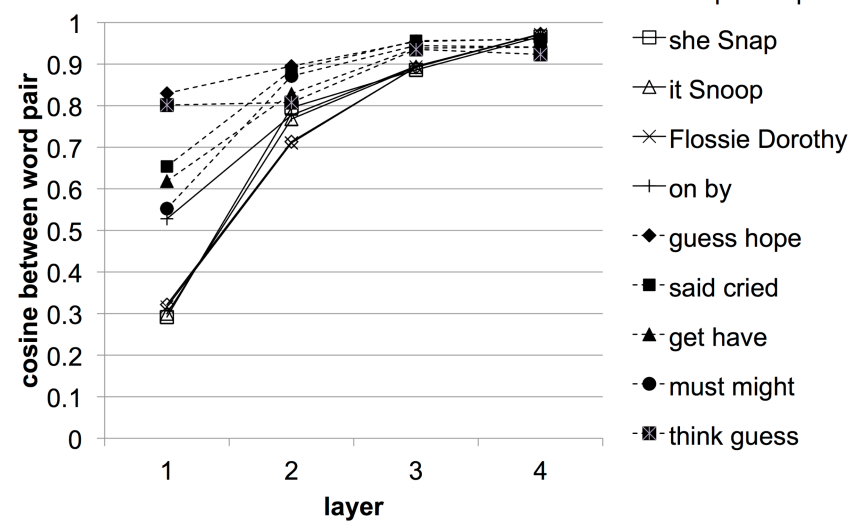

Figure 9.8. Cosine similarities between word pairs across layers in Simulation 4. Word pairs selected to show the effect of each layer of the model. For each layer after the first, we select the 5 word pairs from the top 100 of that layer that increased the most from the previous layer. For contrast, we also select the 5 word pairs from the top 100 of the previous layer that decreased the most by the target layer. 

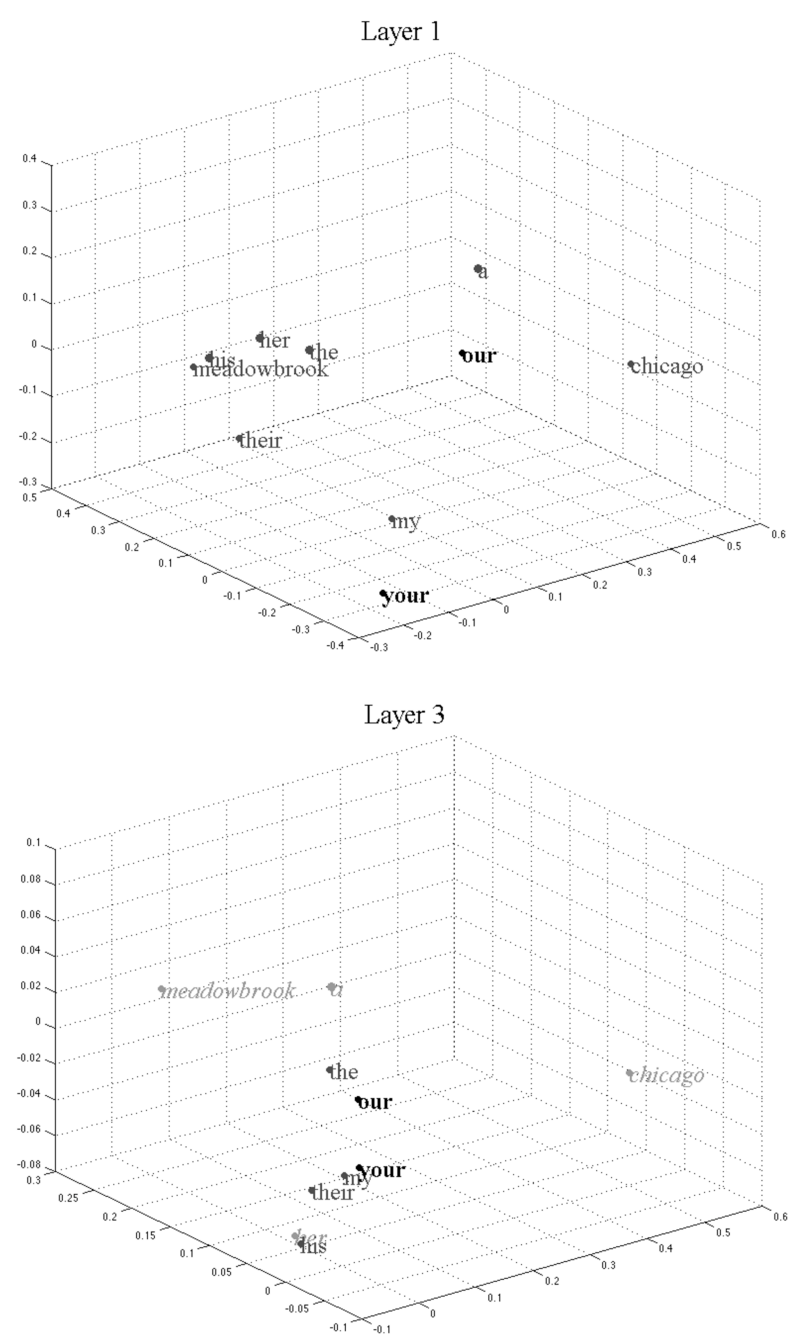

Layer 2

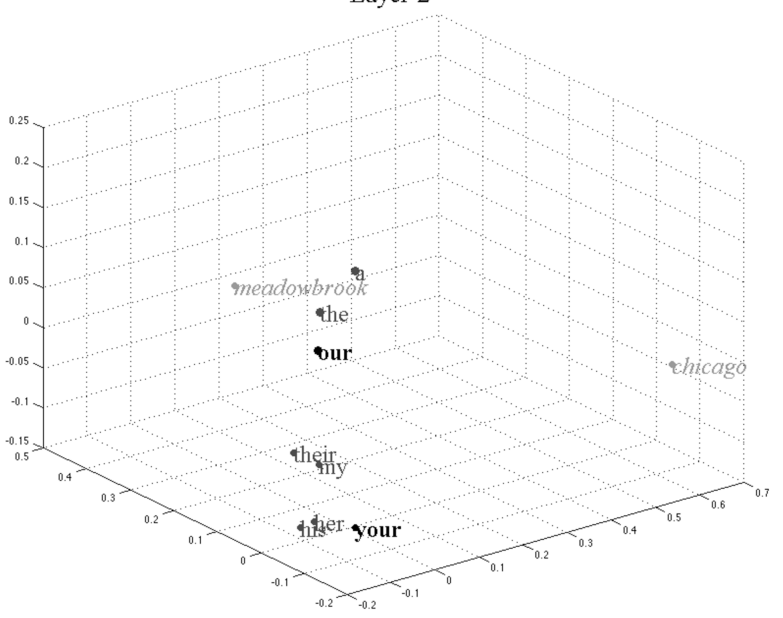

Layer 4

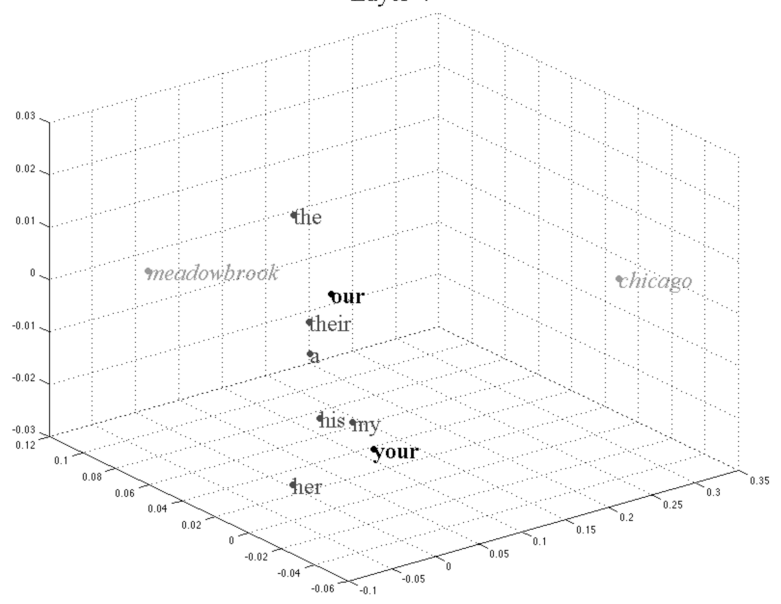

Figure 9.9. Cosine distances for the target words our and your and their five nearest neighbours at each layer in Simulation 4. Label and markers indicate target (black bold), neighbour (grey), and not a neighbour (light grey italics). Compressed to 3 dimensions using multi-dimensional scaling.

In Figure 9, we plot the cosine distances between the possessive determiners our, your, and each of their five nearest neighbours at each layer. Our and your are indicated with black markers and bold text, their neighbours at each layer are indicated with grey markers, and words 
that are not neighbours of my and your at the given layer are indicated with light grey markers and italic text. At Layer 1, our and your are erroneously near neighbours to the cities of Chicago and Meadowbrook. By Layer 2, Chicago and Meadowbrook are no longer near neighbours. The remaining near neighbours are all determiners (a, the, our, their, my your, his, her). Higher layers have little further effect.
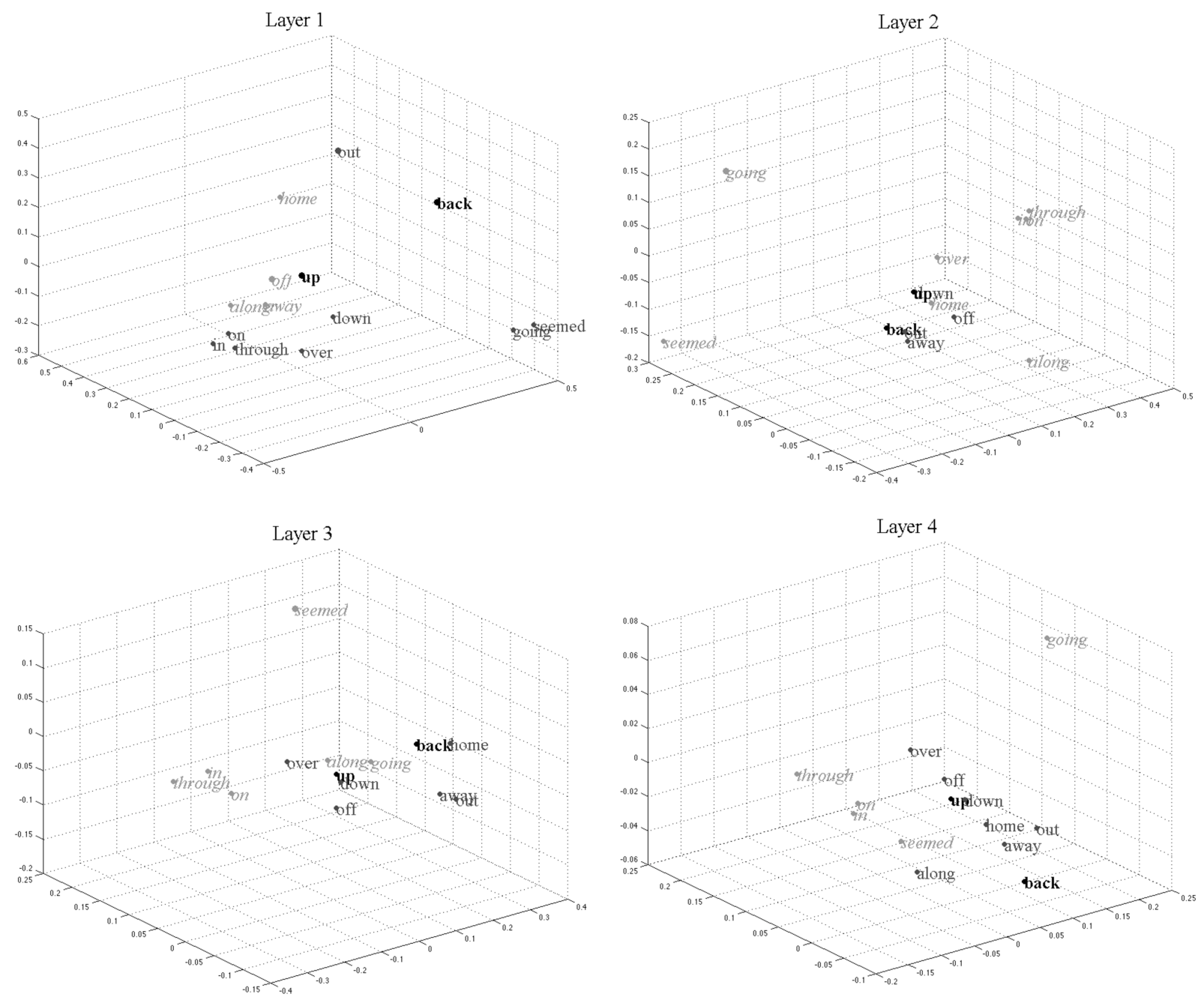

Figure 9.10. Cosine distances for the target words back and up and their five nearest neighbours at each layer in Simulation 4. Label and markers indicate target (black bold), neighbour (grey), and not a neighbour (light grey italics). Compressed to 3 dimensions using multi-dimensional scaling. 
In Figure 9.10 we plot the cosines distances between the directions back and $u p$ and their five nearest neighbours at each layer. At Layer 1, back is near the verbs going and seemed, though is also near the directions down and out. By Layer 2, back and up form a cluster of directions that excludes the verbs from Layer 1. At Layers 3 and Layer 4, the model respectively learns that home and along can also be understood as directions.

\subsection{General Discussion}

The memories of humans are dense jungles, populated by all of the things we have encountered in our lives and thought were important enough to keep in the verdant glades of our mind. To find anything in our memory, we must traverse paths between the things that are important to us. These paths are trampled down into mud by use but are in constant danger of becoming completely overgrown.

The jungle metaphor isn't perfect, but it conveys the core insights of current computational memory models. To retrieve an item from memory, there must be a path to the item from the current stimulus (the retrieval cue). These pathways, called associations, are strengthened by use and weakened by disuse (either through decay or interference, depending on the theory of memory).

The Hierarchical Holographic Model is a memory system that develops shortcuts, more direct paths between related items, at higher layers of the model. For example, for the data set in Simulation 1 (see Table 9.1), to get from birds to eagles using just co-occurrence information requires stepping from birds to fly, fly to skies, skies to soar, and soar to eagles. Taking such a circuitous route through the jungles of memory, we are likely to get lost and never reach our 
destination. The Hierarchical Holographic Model hypothesizes that humans develop shortcut associations between indirectly related items in memory. By increasing the number of layers in the Hierarchical Holographic Model, we can detect associations of arbitrarily high order, that is to say, develop shortcuts been arbitrarily indirectly related items in memory. These shortcut associations may play a critical role in the human ability to understand the world.

In our simulations we have shown that the Hierarchical Holographic Model exploits higher-order associations that the BEAGLE (Jones \& Mewhort, 2007) model of semantic memory cannot. Furthermore, by running the model on a collection of children's novels, we show that exploiting these higher order associations is useful for the purposes of detecting semantic and part of speech information in words.

Chomsky (1956) gave the sentence "Colorless green ideas sleep furiously" as an example of a sentence that is grammatically correct but meaningless. That such sentences can be constructed seems to demonstrate that syntax and semantics are distinct from each other. Chomsky intended the sentence as an argument against statistical models of speech. Unless the sentence "Colorless green ideas sleep furiously" was part of the statistical model's training corpus, the model would neither be able to generate that sentence nor determine that the sentence is grammatical.

Chomsky's observation is certainly true of BEAGLE, the first level of the Hierarchical Holographic Model, which is constrained to first and second order associations. However, we hypothesize that higher levels of the Hierarchical Holographic Model would be able to identify that "Colorless green ideas sleep furiously" is a valid sentence. Even though the model will never have encountered the sentence before, it will have encountered sentences that were likewise 
constructed as "adjective adjective noun verb adverb". At higher layers, the representations for words of the same part of speech will look increasingly alike, such that at high layers of the model, "Colorless green ideas sleep furiously" should look like a familiar sentence. However, testing this hypothesis would require running the Hierarchical Holographic Model on a larger dataset than 15 children's novels. But even given the relatively constrained language experience provided by those novels, the Hierarchical Holographic Model provides evidence that semantics and syntax exist on a continuum and emerge from a unitary cognitive system.

We find evidence that sensitivity to part of speech information, such as the knowledge that our and your are both determiners or was and were are both forms of the same verb, is gained by exploiting higher order associations (see Figure 9.7). We hypothesize that part of speech information, that is to say, how words are used syntactically, is governed by higher-order associations. At first order association, we can detect related topic (e.g., tigers and stripes). At second order association we can detect synonyms (e.g., Mr. and Mrs.). At high orders, we can detect syntactic classes, such as determiners (e.g., our and your). Thus, "purely" syntactic and "purely" semantic associations between words are not different in kind, but differ only in the order of association, that is, the degree of separation between the words in language.

To our knowledge, analyzing the relationships between words in terms of their degrees of separation is a novel approach in psycholinguistics. However, we do not intend orders of association as a construct for language analysis, but as a construct for analyzing the relationships between concepts in memory. We believe that the capacity of the Hierarchical Holographic Model to exploit higher-order associations can be usefully applied to other domains, such as case-based reasoning and analogical reasoning. 
We refer to the layers of representations in the Hierarchical Holographic Model as layers because we were inspired by deep learning neural networks. However, we do not claim that these layers are distinct neural structures. These layers are representations, virtual mental constructs, rather than physical biological constructs. The layers of the model could all be represented within a single fully distributed neural structure. As such there is also no necessary limit to the number of such layers that could exist in the mind, as they are not physical constructs.

\subsection{Conclusion}

We define orders of association as the degree of separation between concepts in memory or words in language. We observe that words with strong first order association are related in topic and words with strong second order association have a strong degree of synonymy. We hypothesize that sensitivity to "purely" syntactic relationships is not different in kind from sensitivity to semantic relationships, but rather relies on exploiting greater degrees of separation between words. We note that computational models of distributional semantics in the literature are only sensitive to first and second order associations. We present a new model of distributional semantics, the Hierarchical Holographic Model, an extension of the BEAGLE (Jones and Mewhort, 2007) model of semantic memory. The Hierarchical Holographic Model is a series of BEAGLE models, such that the vector output of one model feeds in as the vector input to the next model. On simple artificial data sets, we demonstrate that higher layers of the model are sensitive to higher orders of association and improve on the ability of the BEAGLE model to detect categorical relationships. On a data set of 15 children's novels, we demonstrate that higher layers of the model detect syntactic and semantic relationships that the BEAGLE 
model fails to detect. The Hierarchical Holographic Model is not intended as a language model but as a model of memory with the ability to detect more abstract associations. The present work is a proof of concept of the utility of orders of association as a theoretical construct and the Hierarchical Holographic Model as an extension of the BEAGLE model. Exploring further applications of the Hierarchical Holographic Model to language or other domains, such as case based or analogical reasoning, is a matter for future work. 


\section{Chapter 10 Memory Model Framework}

We present further analysis of existing memory models, examining in depth the distinction between hetero- and auto-associative models, the distinction between the concept-based knowledge organization of BEAGLE / HDM / DSHM versus the instance-based knowledge organization of all other memory models, and the question of how to generate decisions and timing from the outputs of memory models. On the basis of this analysis, we propose a theoretical framework that characterizes memory modelling in terms of seven key decisions: (1) choice of data structure, (2) choice of associative architecture, (3) choice of knowledge representation scheme, (4) choice of learning rule, (5) choice of knowledge organization, (6) choice of decision response criteria, and (7) choice of representation scheme.

This framework is both descriptive and proscriptive: we intend to both describe the state of the literature and outline what we believe is the space of possibilities that will be most fruitful in the development of future memory models.

\subsection{Towards Unification}

In Chapter 4, we classified vector-based memory models in the literature as belonging to one of three classes: many-to-one, one-to-one, and many-to-many. In many-to-one models, the memory model stores all experiences as a composite, superimposed on a single data structure in a fully distributed manner. In one-to-one models, the memory model stores each experience individually, as if it were a box on a shelf in a giant warehouse. In many-to-many models, concepts are stored individually and can be understood as points on a hyper-sphere, shifting on 
the surface of a globe of memory and meaning, moving closer or further away from the other concepts on the basis of experience.

In Chapter 5, we presented a proof of equivalence between MINERVA 2 (a one-to-one model) and a Hebbian auto-associative memory implemented as a fourth-order tensor (a manyto-one model). This a proof of equivalence holds true for MINERVA 2 and a number of MINERVA 2 variants. Here we introduced the term memory tesseract to refer to (1) a fourthorder tensor memory system, but also (2) a holographic vector compressed approximation to a fourth-order tensor memory system.

In Chapters 7 and 8, we demonstrated the performance of HDM, a many-to-many model, on the fan effect task and an iterated sequential decision-making task. As we argued in Section 5.7 and as we will discuss in more detail in this chapter, HDM can be understood as a model within our theoretical framework, which is to say, it can be re-implemented as a MINERVA model, or equivalently, a fourth-order tensor memory system, or a holographic approximation thereof.

However, in our arguments that the many-to-one, one-to-one, and many-to-many memory models can all be realized in a single architecture, we have focused on the choice of data structure, but we have glossed over four other important distinctions that we will now discuss: hetero-associative versus auto-associative, error-less versus error-driven learning, the difference between concept-based and instance-based memory and how this affects predictions for the fan effect task, and how the model generates response time predictions. 


\subsection{Hetero-Associative versus Auto-Associative}

In a hetero-associative memory, associations are formed between two different items. In an autoassociative memory, an item forms associations with itself. The proof in Chapter 5 shows that the one-to-one MINERVA 2 model is mathematically equivalent to a many-to-one auto-associative fourth order tensor. This does not show that MINERVA 2 is equivalent in behaviour to the oneto-one models in the literature, as many of those models in the literature are hetero-associative.

This means that in MINERVA, a cue always retrieves a similar memory, or combination of similar memories, and new information is a matter of remembering more detail, whereas in other models, a cue can retrieve completely dissimilar memories that have become associated with that cue through experience.

In practice, the distinction between hetero- and auto-associative makes little difference. Given a pair of items to remember, $\mathbf{x}$ and $\mathbf{y}$, and then tested with the item $\mathbf{x}$, a MINERVA model will retrieve an echo that contains both $\mathbf{x}$ and $\mathbf{y}$. A hetero-associative memory given $\mathbf{x}$ simply retrieves $\mathbf{y}$.

However, modelling a recognition task with an auto-associative memory is more straightforward than with a hetero-associative memory. To test if an auto-associative memory system has studied a pair $\mathbf{x} \mathbf{y}$, one presents the auto-associative memory with the cue $\mathbf{x} \mathbf{y}$, and if that pair is present in memory, the model will retrieve $\mathbf{x} \mathbf{y}$. Performing a recognition task with a hetero-associative memory is not as simple.

HDM behaves like a hetero-associative memory. In Chapter 7, we used HDM to perform a recognition task, specifically, the fan effect task. In order to model a recognition task, HDM converts a cue $\mathbf{a} \mathbf{b}$ into a pair of probes: $\mathbf{a}$ ? and $\mathbf{?} \mathbf{b}$ which, if $\mathbf{a} \mathbf{b}$ is a studied pair, will 
respectively retrieve $\mathbf{b}$ and $\mathbf{a}$. But how should the response from each of the two probes be combined to produce a response from the model? In Chapter 7, we take the mean of the two cosines: the cosine between $\mathbf{b}$ and the response to the probe $\mathbf{a}$ ?, and the cosine between $\mathbf{a}$ and the response to the probe $\mathbf{?}$ b. This may not be the best approach.

Humphreys et al. (1989b) compared the pair recognition behaviour of the MINERVA 2 (Hintzman, 1986), Search of Associative Memory (SAM; Raaijmakers \& Shiffrin, 1981; Gillund \& Shiffrin, 1984), and TODAM (Murdock, 1982) models of memory, as well as Humphreys et al.'s own matrix model. Table 10.1 is a modified version of the table that appears in Humphreys et al. (1989b), extended to include ACT-R, HDM, and the Holographic Exemplar Model (HEM; Jamieson \& Mewhort, 2011).

Table 10.1. Comparison across memory models of matching strengths in a recognition task.

\begin{tabular}{lcccccc}
\hline $\begin{array}{l}\text { Cue } \\
\text { Pair }\end{array}$ & MINERVA 2 & $\begin{array}{c}\text { Matrix or } \\
\text { TODAM }\end{array}$ & SAM & ACT-R & HEM & HDM \\
\hline $\mathbf{x}_{i} \mathbf{y}_{i}$ & $(2 \alpha)^{3}-(2 \beta)^{3}$ & $\alpha^{2}-\beta^{2}$ & $2 a b-2 d^{2}$ & $2 s-2 u$ & $\left(2 \alpha+\alpha^{2}\right)^{3}-\left(2 \beta+\beta^{2}\right)^{3}$ & $2 \alpha$ \\
$\mathbf{x}_{i} \mathbf{y}_{j}$ & $2(\alpha+\beta)^{3}-2(2 \beta)^{3}$ & $2 \alpha \beta-2 \beta^{2}$ & $2(a d+b d)-4 d^{2}$ & $s-u$ & $2(\alpha+\beta+\alpha \beta)^{3}-2\left(2 \beta+\beta^{2}\right)^{3}$ & $2 \beta$ \\
$\mathbf{x}_{i} \mathbf{z}$ & $(\alpha+\beta)^{3}-(2 \beta)^{3}$ & $\alpha \beta-\beta^{2}$ & $(a d+b d)-2 d^{2}$ & $(s-u) / 2$ & $(\alpha+\beta+\alpha \beta)^{3}-\left(2 \beta+\beta^{2}\right)^{3}$ & $\beta$ \\
\hline
\end{tabular}

The analysis in Table 10.1 simplifies the math of the models to capture the essential details of interest. Each memory model has studied a set of $m$ pairs $\mathbf{x}_{i} \mathbf{y}_{i}$ for all $i$ from 1 to $m$. Table 10.1 shows the "matching strength" elicited from each model when presented three different cues: one of the studied pairs $\mathbf{x}_{i} \mathbf{y}_{i}$, an unstudied pair of studied items $\mathbf{x}_{i} \mathbf{y}_{j}, i \neq j$, and a studied item paired with an unstudied item $\mathbf{x}_{i} \mathbf{z}$. All matching strengths in Table 10.1 are expressed as a difference: the matching strength for a pair of unstudied items $\mathbf{w} \mathbf{z}$ is subtracted 
from the matching strength of for each of the three cues. The matching strength for a completely novel cue $\mathbf{w} \mathbf{z}$ acts as a baseline. Subtracting it out simplifies the matching strength expressions by eliminating any terms that are common to all cues, such as baseline activation in ACT-R (see Chapter 7). Thus the matching strengths presented are, to an extent, a measure of the ability of the model to discriminate between a target $\mathbf{x}_{i} \mathbf{y}_{i}$ and foils $\mathbf{x}_{i} \mathbf{y}_{j}, \mathbf{x}_{i} \mathbf{z}$, and $\mathbf{w} \mathbf{z}$.

In Table 10.1, for all vector-based models, $\alpha$ is the mean similarity of matching vectors and $\beta$ is the mean similarity of mismatching vectors. The value of $\alpha$ is around 1 under ideal conditions (minimal interference and good encoding) and $\beta$ should be around 0 .

MINERVA 2 and HEM For MINERVA 2 and HEM, the "matching strength" is the echo intensity for the cue minus the echo intensity for $\mathbf{w} \mathbf{z}$. Echo intensity is commonly used in MINERVA 2 and related models as a measure of familiarity for the purpose of making recognition judgements. Echo intensity, is not, however, the only approach (see Chapter 5 for discussion). Recognition judgements are sometimes made on the basis of the cosine similarity between the vectors for the echo and the probe. Using that technique, the content of the echo rather than the intensity is what matters. But the echo intensity and echo content are calculated in a very similar way, such that Table 10.1 also provides insight into the content of the echo for MINERVA 2 and HEM.

For MINERVA 2, given the cue $\mathbf{x}_{i} \mathbf{y}_{i}$ the echo is dominated by the matching trace $\mathbf{x}_{i} \mathbf{y}_{i}$, which is weighted by $(2 \alpha)^{3}$. Given the cue $\mathbf{x}_{i} \mathbf{y}_{j}$ the echo is dominated by the traces $\mathbf{x}_{i} \mathbf{y}_{i}$ and $\mathbf{x}_{j} \mathbf{y}_{j}$, each weighted by $(\alpha+\beta)^{3}$. Given the cue $\mathbf{x}_{i} \mathbf{z}$ the echo is dominated by the trace $\mathbf{x}_{i} \mathbf{y}_{i}$ weighted by 
$(\alpha+\beta)^{3}$. As such, for the three cues in Table 10.1, the echo for the target cue $\mathbf{x}_{i} \mathbf{y}_{i}$ will be the most similar to the cue and the echo for the foil cue $\mathbf{x}_{i} \mathbf{z}$ will be the least similar to the cue.

The behaviour of HEM's echo is similar to MINERVA 2. However, HEM's traces are constructed differently. MINERVA 2 represents an association between pairs of items by concatenating the two vectors representing the items. HEM represents an association between two items using a combination of addition and convolution. The pair $\mathbf{x}_{i} \mathbf{y}_{i}$ is represented as $\mathbf{x}_{i}+\mathbf{y}_{i}$ $+\mathbf{x}_{i} * \mathbf{y}_{i}$. As a result of using both superposition and binding to construct associations, HEM's matching strength is both a sum and a product of matches: $2 \alpha+\alpha^{2}$ when the cue matches a pair in memory, $2 \beta+\beta^{2}$ where the cue is a complete mismatch, and $\alpha+\beta+\alpha \beta$ in the case of a partial match. HEM's matching strength / familiarity is like a sum of the familiarity values for the autoassociative MINERVA model and the hetero-associative Matrix model.

Matrix and TODAM To measure matching strength for the matrix or TODAM models, a representation of the cue is created by binding the representations of the two items in the pair. For TODAM, this is done using convolution, $\mathbf{x}_{i}^{*} \mathbf{y}_{i}$, whereas for the matrix model the outerproduct is used, $\mathbf{x}_{i} \mathbf{y}_{i}^{\mathrm{T}}$. The matching strength is the similarity, as measured by the dot product, between the cue and TODAM's memory vector or the Humphrey et al. (1989b) memory matrix. TODAM and the matrix model are hetero-associative memories, so, instead of doing a retrieval or a pair of retrievals, measuring the similarity between the cue and the memory vector or matrix serves as a workaround for doing recognition.

Note that TODAM also stores representations of items individually using addition, and not just in convolved pairs, i.e., $\mathbf{x}_{i}+\mathbf{y}_{i}+\mathbf{x}_{i} * \mathbf{y}_{i}$, such that behaviour of the full TODAM model 
may more closely resemble that of HEM. Stored representations of individual items is referred to as item information and stored representations of associations between items, such as pairs, is referred to as associative information (Murdock, 1993). This distinction is present in a number of memory systems, such as TODAM (Murdock, 1993), HEM (Jamieson \& Mewhort, 2011), and others (e.g., Franklin \& Mewhort, 2015).

HDM The matching strength for HDM is the sum of the cosines for the two probes, that is to say, the sum of:

(1) the cosine between the memory vector for one item $\mathbf{m}_{x}$ and the vector for the cue to retrieve that item, $\mathbf{q} ?$, and

(2) the cosine of the memory vector for the other item $\mathbf{m}_{y}$ and the cue to retrieve that other item $\mathbf{q}_{x}$.

In other words, the familiarity $f$ is:

$$
f=\operatorname{cosine}\left(\mathbf{m}_{x}, \mathbf{q}_{? y}\right)+\operatorname{cosine}\left(\mathbf{m}_{y}, \mathbf{q}_{x ?}\right)
$$

The cosines for any unstudied items will be exactly zero in the current version of the model. The matching strengths in Table 10.1 are expressed as a difference between the response to a cue and the response to a pair of unstudied items. Because the cosine for unstudied items is zero, all matching strengths for HDM are subtracted by zero.

The behaviour of HDM on foils (that is, unstudied pairs) was not tested against human experimental data in Chapter 7 and is, at best, questionable as a model of human behaviour. In defence of HDM, Mewhort and Johns (2005) argue that contradiction rather than matching strength is the basis for decisions, and so the matching strengths for foils may not be relevant. 
Nonetheless, HDM's present lack of a tested model for foils is a reason to prefer to adopt a more tried and true model of memory, such as MINERVA or HEM.

The main issue of HDM may be the fact that it lacks item information. HDM is a concept-based memory system: all data is stored as relationships between concepts. Conversely, the other memory systems in Table 10.1 are instance-based: all data is stored as individual experiences. As such, all data stored in HDM is intrinsically associative information, all memories are defined as relationships between items. HDM has no difficulty in rejecting novel pairs of studied items, as what it stores are the pairs rather than the studied items. Conversely, from human performance, as measured by both accuracy and response latency, foil difficulty can be ranked as $\mathbf{x}_{i} \mathbf{y}_{j}>\mathbf{x}_{i} \mathbf{z}>\mathbf{w} \mathbf{z}$, that is, pairs that include studied items are more difficult to reject as foils than pairs that do not include studied items. HDM, at present, does not distinguish between these different types of foils, as it lacks item information.

ACT-R For ACT-R, "signal strength" is the difference between the level of activation of the retrieved chunk and the level of activation of a chunk unrelated to the cue. The strength of association between matching items is $s$ and the strength of association between mismatching items is $u$, such that $s$ and $u$ are analogous to $\alpha$ and $\beta$ in the vector models.

It is unclear whether ACT-R can distinguish between a $\mathbf{x}_{i} \mathbf{y}_{j}$ foil and a $\mathbf{x}_{i} \mathbf{Z}$ foil. According to Anderson and Reder's (1999) model of the fan effect task, given a foil, half of the time ACT-R retrieves the chunk most similar to one of the two items in the pair, and half of the time ACT-R retrieves the chunk most similar to the other item in the pair. Thus, we have assumed that half of the time, ACT-R retrieves an unrelated chunk given the probe $\mathbf{x}_{i} \mathbf{z}$ and half of the time ACT-R 
retrieves $\mathbf{x}_{i} \mathbf{y}_{i}$. We average across these two matching strengths to get $(s-u) / 2$. Under these assumptions, ACT-R most resembles the MINERVA 2 model in its predictions.

SAM For SAM, $a$ is the strength of association between an item and itself, $b$ is the strength of association between an item and its pair, and $d$ is the pre-experimental background association strengths. The $a$ and $b$ in SAM are equivalent to $\alpha$ in the vector models and $d$ is equivalent to $\beta$. SAM essentially behaves like a description of a matrix or TODAM model, though with the ability to independently modulate self-strength and pair-strength, a feature which is also a property of Holographic Exemplar Model (HEM; Jamieson \& Mewhort, 2011), a hybrid of the holographic vector models and MINERVA.

Superposition versus Binding TODAM, HDM, SAM, and the matrix model are the hetero-associative models. MINERVA 2, ACT-R, and HEM are the auto-associative models. The impact of this distinction on matching strength is easiest to see from a comparison of the MINERVA 2 and matrix models in Table 10.1. In MINERVA 2, a matching strength is a sum: $2 \alpha$ if it's a complete match, $\alpha+\beta$ if it's a partial match, $2 \beta$ if it's a complete mismatch. In the matrix model, a matching strength is a product: $\alpha^{2}$ if it's a complete match, $\alpha \beta$ if it's a partial match, $\beta^{2}$ if it's a complete mismatch. This difference in matching strength arises from the choice of using either a binding operation, such as convolution or the outer-product, or a superposition operation, such as vector addition or concatenation. For hetero-associative models, a binding operation is used, and matching strengths are a product. For auto-associative models, a superposition operation is used, and matching strengths are a sum. Which fits human data better? 
It's worth noting that in ACT-R, activation, which is what the matching strength in Table 10.1 is based on, is a sum, as in other auto-associative models like MINERVA. However, ACT-R response latencies are a function of the product, as shown in Section 7.4. This is due to a mathematical trick: reaction time is $T=I+F e^{-A}$ where and $I$ and $F$ are fitting parameters and $A$ is the activation. By setting the association strengths to be the natural logarithm of the desired values, the sum becomes a product: $e^{\ln (x)+\ln (y)}=x y$. Thus, in terms of its reaction time predictions, ACT-R behaves like a hetero-associative model.

In the ACT-R model of the fan effect, reaction time is a function of the product of the fans of the items rather than the sum. This is supported by human data: the min effect is the finding that in the fan effect, a 1:3 probe (i.e., a probe where one item of the pair occurs once in the study set and the other occurs three times) is faster to make recognition judgements on than a 2:2 probe, even though the sum of their fans is the same (Anderson \& Reder, 1999). Conversely, the products are $1 \times 3=3$ and $2 \times 2=4$.

Pairs represented as a superposition of their items provides item information and pairs represented as a binding of their items provides associative information. There is some theoretical consensus that both types of information are necessary: both types are present in TODAM (Murdock, 1993), HEM (Jamieson \& Mewhort, 2011), and other models (e.g., Franklin \& Mewhort, 2015). We suggest that a unified memory model would need to incorporate both kinds of information. 


\subsection{Errorless versus Error-driven Learning}

In our claim in Chapter 8, that HDM can account for the type of learning and memory generally attributed to a procedural memory system, we glossed over a substantive difference between ACT-R's declarative and procedural memory systems. ACT-R's declarative memory uses errorless learning: associations between things in the model's environment are added to the declarative memory store as chunks of information. Conversely, ACT-R's procedural memory uses error-driven learning, that is, reinforcement learning techniques. Walsh and Anderson's (2011) model of the decision-making task described in Chapter 7 used the procedural memory system to learn the task. Specifically, they used temporal difference learning, a learning technique that makes predictions, then adjusts the model that made those predictions based on the difference between what was predicted to happen and what actually happened.

MINERVA-AL is a variant of MINERVA that uses reinforcement learning. MINERVAAL makes and corrects predictions to capture numerous associative learning phenomena from both the animal and human learning literature (Jamieson, Crump, \& Hannah, 2012). MINERVAAL demonstrates that error-driven learning does not require a distinct memory architecture, as aside from how it learns, MINERVA-AL is otherwise a standard MINERVA model.

Are both errorless and error-driven learning rules required to model human memory? Or can human memory be modelled using error-driven learning alone? If both types of learning are needed, how do they relate to each other, and how does the model select which type of learning to use?

We take MINERVA-AL and our work with HDM in Chapter 8 as evidence that a distinct procedural memory system or error-driven learning store is unnecessary. It is possible that 
errorless and error-driven learning are two learning strategies employed in different circumstances by a unitary memory system. Exploring through simulations and modelling the distinction between error-driven versus errorless learning falls beyond the scope of this thesis, but we discuss the distinction here for the sake of completeness in our survey and analysis of the memory models in the literature and the hurdles that must be overcome en route to a unified theory of memory.

\subsection{Concept-based versus instance-based models and the placeholder vector}

Let us compare the Holographic Exemplar Model (HEM; Jamieson \& Mewhort, 2011) with the

BEAGLE / DSHM / HDM models. There are strong similarities between these models. Both

HEM and the BEAGLE variants use holographic vectors to represent knowledge. Both HEM and BEAGLE are multi-vector models. There are, however, three important differences: HEM uses the MINERVA retrieval mechanism, aggregating across all vectors to construct an echo. Conversely, BEAGLE does not have a retrieval mechanism. HDM and DSHM select the nearest memory vector(s) to the probe for recall or use a mean of the distance to the nearest memory vectors for matching strength / familiarity in recognition.

(2) HEM stores one vector per episode or event whereas BEAGLE stores one vector per concept or item. For this reason, we refer to HEM as a one-to-one model and BEAGLE and variants as many-to-many models. HEM stores one vector for one episode, whereas in BEAGLE and variants each episode is distributed across many vectors, and each vector stores many episodes. 
HEM structures knowledge in terms of episodes and the relationships between items within an episode / instance, whereas BEAGLE and variants structure knowledge in terms of items / concepts and the relationship each item has to all other items. We thus refer to BEAGLE / DSHM / HDM as concept-based models and all other memory models, including HEM, as instance-based models.

With regard to the first distinction, there may be good reason to prefer the MINERVA retrieval mechanism over the HDM / DSHM retrieval mechanisms, as we discussed in the previous section. Fortunately, it is not difficult to implement a version of HDM / DSHM / BEAGLE that uses MINERVA's retrieval mechanism. For example, Chubala, Johns, Jamieson, and Mewhort (2016) use a MINERVA model loaded with BEAGLE vectors to emulate human performance on an implicit learning task. The model uses echo intensity calculated by aggregating across BEAGLE vectors to discriminate between targets (words that conform to the implicit rule) and foils (words that violate the implicit rule). In the next section, we demonstrate performance on the fan effect for an HDM model reimplemented in the MINERVA architecture.

With regard to the second distinction, as we argued in Chapter 5, the many-to-one, oneto-one, and many-to-many data structures can all be understood as instantiations of the same memory theory, where a one-to-one is exactly equivalent in behaviour to a many-to-one model, and a many-to-many can be understood as a collection of the echoes from one-to-one or many-toone models. The critical difference between these models is practical. On a serial computer, for small scale tasks a one-to-one may be more computationally efficient than a many-to-one model and for large scale tasks a many-to-many model may be more computationally efficient. 
This leaves the third distinction between HEM and the BEAGLE / DSHM / HDM models. In HEM and other MINERVA models, knowledge is encoded as descriptions of episodes. In BEAGLE and variants, knowledge is encoded as relative to a concept. In BEAGLE and variants, knowledge about an item can be understood as the sum of all questions to which that item is the answer. This can be accomplished by using the placeholder vector, though there are other ways of encoding knowledge as relative to an item (e.g., Sahlgren et al., 2008). The placeholder vector serves as the question mark for the questions stored in memory, or to put it another way, the blank to be filled in by the item in question.

Using a placeholder vector allows a memory system to more easily identify higher-order associations (see Chapter 9). Consider, for example, the sentences "airplanes fly through skies" and "airplanes soar through skies". Using a MINERVA model without using the placeholder vector, to note that $f l y$ and soar are similar, we need to cue memory with " _ fly _ _", retrieve "airplanes fly through skies", cue memory with "airplanes_through skies", and then retrieve "soar" from the resulting mish-mash of the two sentences in the echo. When using the placeholder vector, the memorial representations of soar and fly will be highly similar, such that once you have retrieved the memory vector for soar, it's a simple manner to retrieve the memory vector for $f l y$.

The biggest difference is one of emphasis: A concept-based memory system is designed to learn about the items, whereas an instance-based memory system is designed to record and retrieve experiences. However, these two classes of models do seem to produce different predictions for human behaviour, as we discuss in the next section. 


\subsection{HDM, MINERVA and the Fan Effect}

In Chapter 7, we compared performance of HDM and ACT-R DM on the fan effect. Here we extend that comparison to include MINERVA 2. We find that MINERVA 2 predicts the opposite of the fan effect result. According to MINERVA 2, as fan increases, recognizing the target should become easier rather than harder.

As our purpose here is to compare models to each other rather than to human data, we do not use response time predictions. Translating the result produced by each model into a response time involves a number of fitting parameters that can obscure the similarities and differences between models. For this reason, we compare the matching strengths of the models on the fan effect task, as in Section 10.2.

For MINERVA 2, there are two ways to compute familiarity: (1) echo intensity and (2) similarity between the echo and the probe. As we discussed in Chapter 5, these two methods are, mathematically, almost identical. What does make a drastic difference is whether or not the matching strength is normalized. Typically, echo intensity is not normalized whereas the similarity between the echo and the probe is normalized. To make a pure comparison, we compute familiarity as the similarity between the probe and the echo, and compare a MINERVA 2 model that normalizes the similarity (i.e., computes similarity as the cosine) versus a MINERVA 2 that does not (i.e., computes similarity as the dot product).

Figure 10.1 shows simulations for HDM and four different MINERVA models. All models use the same set of vectors. Vectors have 2048 dimensions. Values for the vectors are sampled from a normal distribution with a mean of zero and a variance of $1 / 2048$. The vectors are then normalized to have a mean of exactly zero and a magnitude of exactly one. 


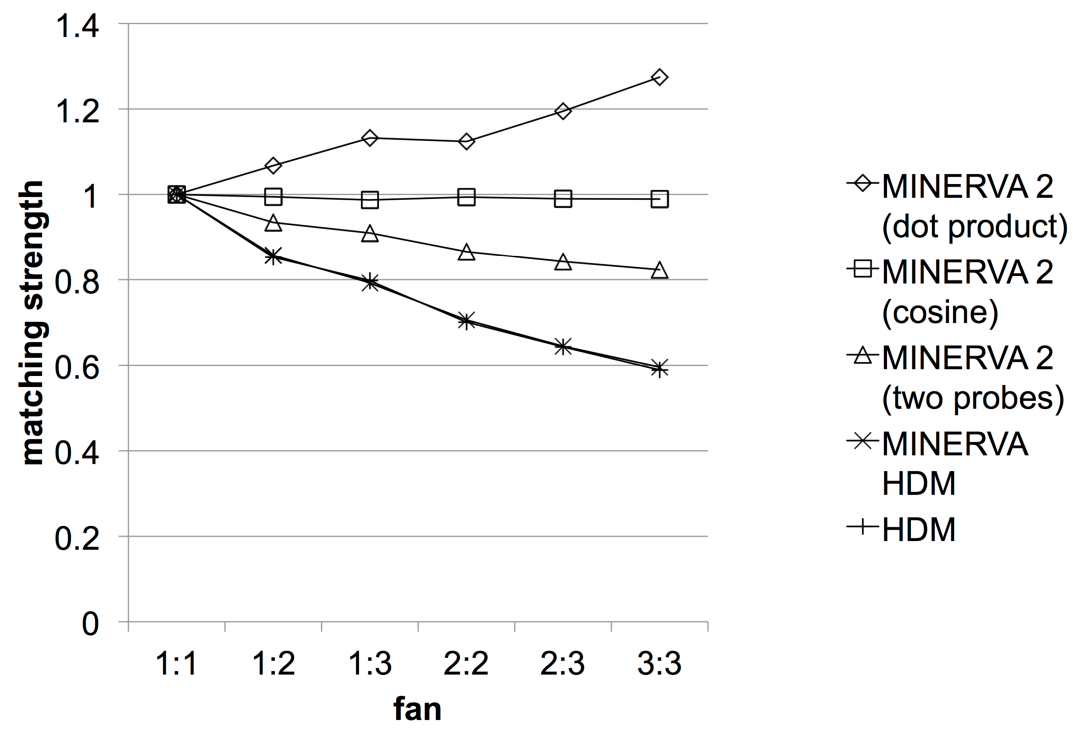

Figure 10.1. Simulated performance of memory models on the fan effect, $n=2048$.

We find that, as in Chapter 7, HDM predicts lower matching strength with larger fan. Conversely, the MINERVA 2 that uses the dot product (no normalizing) predicts higher matching strength with larger fan. This prediction is not wrong exactly, as it makes intuitive sense and would be correct in other experiments. If we understand the matching strength as a familiarity score, the $3: 3$ test item is going to be very familiar, as we have seen both of the words in the test item three times each in the study set. MINERVA 2 is a successful model of recognition tasks where judgements can be made on the basis of familiarity. However, for the fan effect task, participants cannot discern targets test items from foils on the basis of familiarity. Participants need to recall the studied pair to confirm or reject the test item.

The MINERVA 2 that uses the dot product can be understood as the standard MINERVA 2 model for recognition. The MINERVA 2 that use the vector cosine (normalized dot product) can be understood as the standard model for recall. By normalizing the matching strength, we 
eliminate the effect of increased familiarity. Unfortunately, this does not produce the fan effect result. From the simulation, the matching strength looks to be constant across the different fans. Why does MINERVA 2 fail to predict the fan effect? The failure of MINERVA 2 to predict the fan effect is not an inherent property of the MINERVA architecture. We re-implement HDM in a MINERVA architecture to create MINERVA HDM and find that HDM and MINERVA HDM make the same prediction (see Figure 10.1), with minute differences between the two models due to noise.

In MINERVA HDM, for each pair of items in the study set, $\mathbf{x}_{i} \mathbf{y}_{i}$ for $i=1$ to $m$, where $m$ is the number of studied items, we add to memory two traces, one for item $\mathbf{x}_{i}$ and one for item $\mathbf{y}_{i}$,

$$
\mathbf{M}_{i}=\mathbf{M}_{i-1}+\left(\mathbf{x}_{i} \mid \boldsymbol{\Phi} * \mathbf{y}_{i}\right)+\left(\mathbf{y}_{i} \mid \mathbf{x}_{i} * \boldsymbol{\Phi}\right)
$$

where $\mid$ is concatenation, $\mathbf{\Phi}$ is the placeholder vector, and $\mathbf{M}$ is the memory table or tesseract. Given a test item $\mathbf{x}_{j} \mathbf{y}_{j}$ in HDM, MINERVA HDM recalls two echoes, one for the probe $\left(\mathbf{z} \mid \mathbf{x}_{j}\right.$ * $\boldsymbol{\Phi})$, i.e., "what was paired with $\mathbf{x}_{j}$ ?", and one for the probe $\left(\mathbf{z} \mid \mathbf{\Phi} * \mathbf{y}_{i}\right)$, i.e., "what was paired with $\mathbf{y}_{j}$ ?" where $\mathbf{z}$ is a vector of $n$ zeros, $n=2048$ for the simulations in Figure 10.1. The cosine is then computed between first half of each echo and the correct answers to those questions: $\mathbf{y}_{j}$ and $\mathbf{x}_{j}$ respectively.

Is HDM's (and MINERVA HDM's) success at modelling the task a property of its concept-based knowledge organization, or is it a property of the fact that HDM makes two recalls instead of one? To test this, we simulated a variant of MINERVA 2 that makes two recalls instead of one. Given the test item $\mathbf{x}_{j} \mathbf{y}_{j}$, for the first recall, the model uses the probe $\left(\mathbf{x}_{j} \mid \mathbf{z}\right)$ and, for the second, the model uses the probe $\left(\mathbf{z} \mid \mathbf{y}_{i}\right)$. Matching strength is the mean of the cosine between each echo and the test item $\mathbf{x}_{j} \mathbf{y}_{j}$. We find that the MINERVA 2 that makes two recalls 
correctly predicts that for higher fans, matching strength will be lower (see Figure 10.1). Thus, we can infer that HDM's success at the task is a property of making two separate recalls.

To model the fan effect task, the model must make two separate recalls. Given the strong mathematical similarities between the memory models, we can expect this result to generalize to many of the other memory models. If the models are correct, this means that when given a test item $\mathbf{x}_{j} \mathbf{y}_{j}$, human participants choose either $\mathbf{x}_{j}$ or $\mathbf{y}_{j}$ and use that item as a cue to memory. What the participant recalls from memory is then compared against the other item, either $\mathbf{y}_{j}$ or $\mathbf{x}_{j}$, and if it is a match, the participant decides that the pair appeared in the study set. It could be that any individual participant makes only one of these two possible recalls and the fact that response times is a function of the fans of both $\mathbf{x}_{j}$ and $\mathbf{y}_{j}$ is an artifact of averaging across participants.

That participant are doing a recall for each item does not seem immediately implausible. Kwok (2016) and Kwok, West and Kelly (2015) argue that evidence from more complex variants of the fan effect tasks support the hypothesis that participants only use one of the available cues at a time to make a retrieval, even when it would seem to be beneficial to use both cues.

If individual response times are a function of the fan of only one item in each test pair, we should expect to find two clusters of response times for each test pair with unequal fans: one cluster that is a function of the smaller fan and one cluster that is a function of the larger fan. Testing this empirical prediction of the two retrievals model is a matter for future work.

However, this account does raise the question, why wouldn't humans exploit both items in a pair as cues to memory? In MINERVA 2, why does the fan effect disappear when both items are used simultaneously in the probe? 
Table 10.2. Matching strength for models of the fan effect task, assuming ortogonal vectors.

\begin{tabular}{|c|c|c|c|c|}
\hline $\begin{array}{l}\text { MINERVA } 2 \\
\text { (dot product) }\end{array}$ & $\begin{array}{l}\text { MINERVA } 2 \\
\text { (cosine) }\end{array}$ & $\begin{array}{l}\text { MINERVA } 2 \\
\text { (two probes) }\end{array}$ & HDM & ACT-R DM \\
\hline \multirow[t]{2}{*}{$1+\frac{f_{1}+f_{2}-2}{16}$} & $1+\frac{f_{1}+f_{2}-2}{16}$ & $\sqrt{\frac{f_{1}+1}{2 f_{1}}}+\sqrt{\frac{f_{2}+1}{2 f_{2}}}$ & $\frac{1}{\sqrt{f_{1}}}+\frac{1}{\sqrt{f_{2}}}$ & $\ln \left(\frac{1}{f_{1}}\right)+\ln \left(\frac{1}{f_{2}}\right)+2.9$ \\
\hline & $\frac{96+15 f_{1}+15 f_{2}+f_{1}^{2}+f_{2}^{2}}{128}$ & 2 & 2 & 3 \\
\hline
\end{tabular}

To gain a deeper understanding of the behaviour of the models, we perform a mathematical analysis. In Chapter 7, we found that in HDM, a test item with a fan of $f$ produces a cosine of $f^{-0.5}$, i.e., one over the root of the fan. To compute matching strength in HDM, we take the mean of the cosines for the two items in the pair. We extend our mathematical analysis in Chapter 7 of ACT-R DM and HDM to include the MINERVA models in Figure 10.1.

When using approximately orthogonal random vectors, using a smaller dimensionality or larger study set increases noise, but does not systematically bias the matching strength. If we assume perfectly orthogonal vectors, the matching strength for a test pair that was in the study set is given by the equations in Table 10.2, where $f_{1}$ and $f_{2}$ are the fans of the first and second items in the test pair. MINERVA HDM is identical to HDM in matching strength and so is not given a separate entry in the table. Figure 10.2 uses the equations in Table 10.2 to compute the "true" noise-free matching strengths of the models. The calculated matching strengths for the MINERVA and HDM models are nearly identical to the simulated strengths in Figure 10.1.

From the equations in Table 10.2, we see that the matching strengths for MINERVA 2 (dot product) grows linearly with the sum of the fans. As noted before, this would not be an 
unreasonable prediction if we were modelling a task where people make judgements on the basis of a vague sense of familiarity.

Now that we can compute the exact values for the matching strengths for MINERVA 2 (cosine), we find that the model is not constant across different fans. In particular, the model predicts that the most difficult test items is the $1: 3$ fan, with a matching strength of 0.988 , in almost invisible contrast to the 0.990 or more matching strength of the other test items.

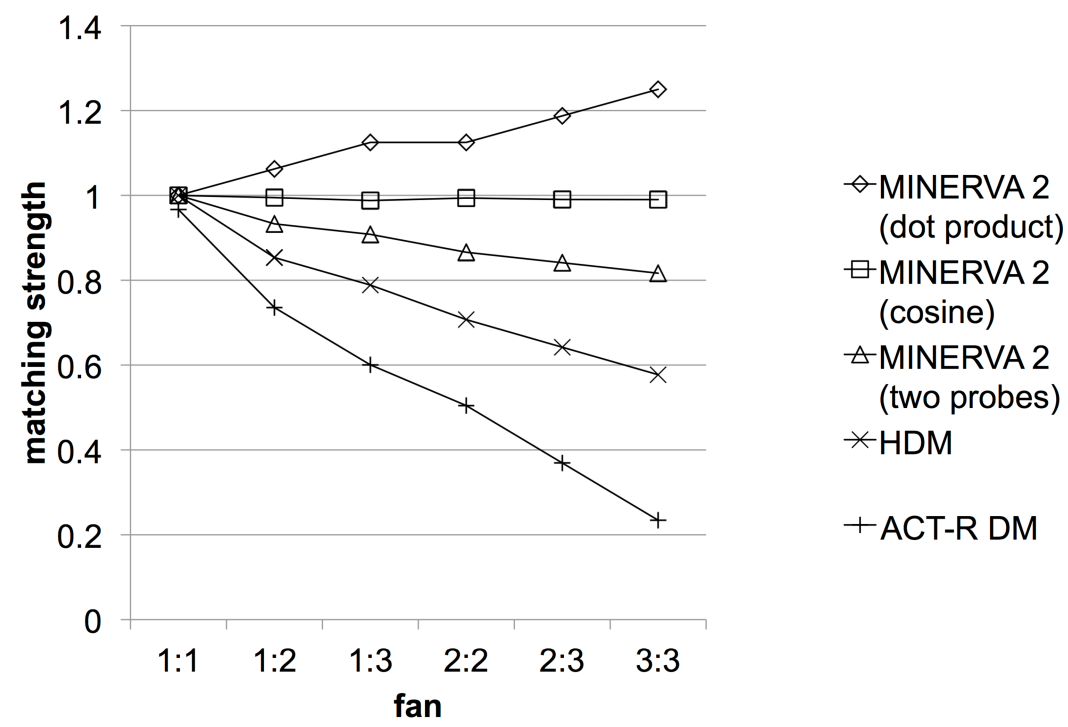

Figure 10.2. Analytic performance of memory models on the fan effect, computed from equations in Table 10.2. Assumes perfectly orthogonal vectors for MINERVA and HDM.

Why is matching strength in MINERVA 2 (cosine) lowest for the 1:3 fan rather than the 3:3 fan? A test item that was in the study set and has a 1:3 fan activates the traces for three studied items in memory: the target item and two lures. Let's say the test item is the personlocation pair hippie-church and that in the study set hippie occurs thrice and church once. In the study set is the pair hippie-church as well as hippie-bank and hippie-park. Given the test item hippie-church the pairs hippie-park, hippie-bank, and hippie-church are activated in memory. The 
echo retrieved will be the sum of these three memory traces, plus some noise. The person part of the echo will be clearly hippie, but the location part of the echo will be a muddled mess of park, bank, and church, lowering the similarity of the probe to the echo overall.

Conversely, for a studied test item with a 3:3 fan, the echo is a composite of five memory traces: the target and four lures. However, due to the interaction between between these memory traces, the echo is clearer than for the 1:3 fan. Let's say that the test item is hippie-park, and in the study set are the items hippie-park, hippie-church, and hippie-bank, as well as captain-park and fireman-park. All of these traces are activated by the probe hippie-park. In the echo, the person will be strongly hippie and weakly captain and fireman, and the location will be strongly park and weakly church and bank. As such, the probe hippie-park will strongly resemble the echo.

So, for MINERVA, the additional items activated in memory in the 3:3 fan compared to the 1:3 fan serve only to reinforce the presence of the target in the echo, which improves similarity to the probe. Conversely, in people, having more lures in memory to sort through makes recall of the target harder.

Thus, the reason that doing two separate recalls fixes the MINERVA 2 model of the fan effect is because it eliminates the mutual reinforcement between the two items in the test pair that makes the 3:3 test pair easier than the 1:3 test pair. If the MINERVA 2 model is correct, participants strategically adopt a strategy of making two recalls because the nature of the foils in the test set make it so that they cannot rely on judgements of familiarity elicited by a single holistic recall. 
Our original intent with comparing MINERVA 2 to HDM on the fan effect task was to examine differences in the behavioural predictions of the concept-based versus instance-based models discussed in the last section. This result could be taken as adjudicating in favour of concept-based models. However, whenever tackling such broad theoretical questions, one should rarely expect to achieve a decisive demonstration for or against from a single result. Within the scheme of either concept-based or instance-based memory models there is an infinite space of different possible models. As already demonstrated, MINERVA 2 can be made to do this task by using two separate recalls. By diagnosing the source of the problem, the mutual reinforcement from the two items, it may be possible to propose yet other methods of getting MINERVA 2 to do the task.

In summary, concept-based memory models (BEAGLE / DSHM / HDM) and instancebased memory models (MINERVA and others) make different predictions on the fan effect task. From close analysis, we discover that this is due to HDM and DSHM using two probes to make a retrieval from memory, whereas for MINERVA and similar models, using the two items in the pair as a single probe produces a mutual reinforcement effect that eliminates the fan effect. If MINERVA uses two probes instead of one, MINERVA predicts the fan effect.

\subsection{Generating Response Time Predictions}

Cognitive models live and die by their ability to model and predict human experimental data.

Much of the experimental data collected is reaction time data. How long does it take the model to recall something? How long does it take for the model to decide if a cue is familiar or unfamiliar? 
In our proposed memory tesseract model, retrieval occurs over a series of iterations as the echo is used as a new cue, which in turn, retrieves a clearer echo. Alternatively, if the memory tesseract is implemented as a neural model, as in Chapter 6, the model is a recurrent network that operates in continuous time rather than in discrete iterations. Either way, over time, discrete or continuous, the model retrieves progressively clearer echoes from memory.

Time to clean up the echo is not the only candidate for the time variant process that accounts for variability in response time in memory tasks. The Iterative Resonance Model (Mewhort \& Johns, 2005) suggests sharpening, a process of incrementally increasing the exponent in a MINERVA model. However, as we argue in Chapter 5, if we understand MINERVA as a memory tesseract, changing the exponent is not a permissible operation. It also seems counter-intuitive to suggest that memory has a sharpness dial that can be adjusted at will. Why would the dial ever be set at less than perfectly sharp?

Cox and Shiffrin (2012), who use a variant of the REM (Shiffrin \& Steyvers, 1997), suggest sampling of features from the probe as the time variant process. Again, this seems counter-intuitive, as it suggests that we are slow to recall some things because we are slow to perceive them correctly. For these reasons, we prefer time to clean up the echo as the time variant process.

At some point, a decision needs to be made on the basis of this retrieved information and the model produces a response. In an actual brain, this decision to act is made by whatever part of the brain needs to use the retrieved information. In a memory model, the only part of the brain being modelled is the memory system itself. As such, the decision is made by a set of criteria that act as a stand-in for those other systems, which are not modelled. As a result, these criteria are a 
somewhat artificial imposition on the model, magic parameters that turn a theory of memory into a system that produces reaction time data.

In principle, integration into a larger cognitive system could help absolve memory models of their need for somewhat arbitrary criteria. ACT-R, unfortunately, treats time to retrieve information from memory as something that is purely internal to the memory model, rather than arising from the interaction between the memory retrieval process and an external decision process.

In a recognition task, the decision that a cue is familiar can be modelled by measuring the similarity between the cue and the echo retrieved in response to the cue. The decision that a cue is unfamiliar has, however, proven more difficult to model. Mewhort and Johns (2005) argue that decisions to reject a cue as unfamiliar is dependent not on similarity but on contradiction. Both Mewhort and Johns's IRM model and Johns, Jones, and Mewhort's (2012) RSS model use contradiction to reject foils, though the two models do not agree on how contradiction ought to be measured.

Part of the reason that criterion setting remains a black art in memory modelling is because it is heavily influenced by the particulars of the task and the strategy that the experimental participant uses to complete the task. Rutledge-Taylor et al. (2014) make an analogy between rejecting foils and cooking popcorn. When cooking popcorn, you learn roughly how much time passes between one kernel popping and the next. If more than the expected amount of time has passed since the last pop, you may judge that the popcorn is finished. Likewise, participants may learn how long it usually takes for them to recollect something in a 
memory task. If they have failed to recollect the cue in about that period of time, they may judge that the cue is a foil.

In short, reaction times in a memory model are a Gordian knot that tangles together participant strategy, the passage of time, and the quantity and quality of the information being retrieved from memory. By necessity, this aspect of memory models may remain ad hoc for quite some time.

\subsection{Framework For Computational Models of Human Memory}

In vector-based models of memory, a to-be-remembered item is represented as a vector. This is convenient because it allows items to be treated as complex entities with internal features and also allows for errors and fuzzy composites of items to be constructed. Vectors are also amenable to implementation in neural models. There are thus good reasons to prefer to use vectors as a means of representing items in memory.

In much of our discussion of vector-based memory models, we have focused on the MINERVA 2 model and, more broadly, the MINERVA class of memory models. Hintzman (1986, p. 411) summarizes MINERVA 2's key assumptions:

(1) only episode traces are stored in memory,

(2) repetition produces multiple traces of an item,

(3) a retrieval cue contacts all traces simultaneously,

(4) each trace is activated according to similarity to the cue,

(5) all traces respond in parallel, retrieved information reflects their summed output.

Through mathematical, comparative analysis of memory models, we have found that most of the memory models can be understood as instantiations of this framework. Memory models that 
violate these assumptions, such as the many-to-many models HDM (Kelly et al., 2015), DSHM (Rutledge-Taylor et al., 2014), and REM (Shiffrin \& Steyvers, 1997) can be understood as higher level descriptions that can be translated into a model that adheres to this framework, as argued in Chapter 5. ACT-R DM (Anderson \& Lebiere, 1999) in turn, can be understood as a higher-level description of HDM, as argued in Chapter 7.

Within this framework of assumptions, there are seven important decisions to be made:

(1) Choice of data structure: MINERVA memory table, a second, third, or fourth order tensor, a the holographic approximation thereof based on Levy and Gayler's model (2009), or a BEAGLE / DSHM / HDM memory table.

(2) Choice of association: Hetero-association or auto-association.

(3) Choice of representation scheme: How vectors are constructed and combined.

(4) Choice of learning rule: Errorless Hebbian learning, or prediction error-driven learning.

(5) Choice of knowledge organization: To use a placeholder vector or not?

(6) Choice of time variant process: Is it the echo or the probe that determines time?

(7) Choice of response decision criteria: At what point does the model respond?

We discuss each of the seven decisions in what follows.

1. Choice of data structure The choice of data structure is largely pragmatic. The MINERVA memory table is mathematically equivalent to the auto-associative fourth order tensor memory (i.e., memory tesseract). If the number of memory traces $m$ is less than the cube of the vector dimensionality $n$, that is, $m<n^{3}$, then the memory table is more efficient. For $m>n^{3}$ the fourth order tensor is more space efficient and more efficient for retrieval from memory, but $n^{3}$ 
times slower for storage. If the sign of the similarity between vectors is unimportant (i.e., negative similarities are not used in the model) one could use a third order memory tensor instead, in which case the third order tensor (i.e., memory cube) is more space efficient and more time efficient for retrieval for $m>n^{2}$, though $n^{2}$ times slower for storage. If one is willing to forego the nonlinearity introduced by the exponent altogether (i.e., use an exponent of one), a matrix implementation is more space efficient and retrieval time efficient for $m>n$ but is $n$ times slower to implement storage.

The holographic approximation is approximately equivalent to either the table or tensor implementation. The holographic approximation uses $p$ memory vectors and is more space efficient for $p<m$ and more time efficient for retrieval for $p \log (n)<m$, but is $p \log (n)$ less time efficient for storage. Alternatively, for large $m$ it may be more efficient to use a memory table implementation of a many-to-many model such as HDM, where $m$ is reduced from the number of experiences that the model has had to the number of unique concepts in its experience.

All of these time efficiency considerations assume a serial computer. In a neural model, storage and retrieval would be implemented in constant time. Space efficiency considerations remain an issue, as higher order tensors may require more neurons (see Chapter 6).

How a new memory is added depends on the choice of data structure. If the memory system is HDM, the experience vector is added to the memory vectors of each constituent concept. If the memory system is a MINERVA table, the experience vector is instantiated as a new row in the table. If the memory system is a tensor, then $\mathbf{M}_{t+1}=\mathbf{M}_{t}+\mathbf{x}$, where $\mathbf{M}$ is the memory tensor and $\mathbf{x}$ is the vector for the experience, and $t$ is the time. If memory system is the 
holographic approximation to the tesseract, then $\mathbf{m}_{i, t+1}=\mathbf{m}_{i, t}+\left(\mathbf{P}_{i, 1} \mathbf{x}\right) *\left(\mathbf{P}_{i, 2} \mathbf{x}\right) *\left(\mathbf{P}_{i, 3} \mathbf{x}\right) * \mathbf{x}$ for all $1<i<p$, where $p$ is the number of memory vectors.

To produce reaction times, we need the memory system to produce time varying responses. To facilitate this, the model should be recurrent. A disadvantage of the table or the tensor is that the echo rapidly stabilizes, such that, in an iterated version of the model, given a typical experimental task, the echo will stabilize after one or two iterations, which makes for a rather uninteresting reaction time graph.

In the holographic approximation to the memory tesseract, the echo stabilizes more slowly, and the process is more error prone, which may allow for a model that is better able to capture human response time and accuracy data.

2. Choice of association The choice between a hetero-associative model and an autoassociative model is a false one. The correct answer is both hetero-associations and autoassociations are useful tools for constructing a memory model. The choice can be made at two levels:

(1) at the level of the architecture of the model

(2) at the level of the representations of the model.

At the level of representation, both auto-associations and hetero-associations can be encoded in a vector by using concatenation, convolution, etc. Both forms of association can and should be used to represent a stimulus as appropriate for the task.

At the level of the architecture of the model, we note, as discussed earlier in the chapter, that recall can be implemented by either a hetero-associative architecture or by an auto- 
associative architecture that encodes hetero-associative representations. There is no difference between cueing with $a$ and recalling $b$ in a hetero-associative model versus cueing with an incomplete stimulus $a$ and retrieving a completed stimulus $a b$ in an auto-associative model.

How a hetero-associative architecture performs a recognition task is less well defined than for an auto-associative architecture. This is because recognition is fundamentally a task of giving the model a cue and seeing if you get something like that cue back again.

In neural terms, a hetero-associative architecture is a set of connections between two neural groups that translates a signal into another signal. As such, hetero-associative architectures are pervasive in the brain, though they are arguably not memory systems at all so much as they are translation systems. Conversely, an auto-associative architecture is a recurrent neural loop that acts as an error-correcting buffer for holding a standing pattern of neural activation, as illustrated in Chapter 6.

3. Choice of representation scheme Vector-symbolic architectures provide a means by which memory models can store representations of arbitrarily complex associations between items. We have focused our discussion on holographic reduced representations (Plate, 1995), or holographic vectors, but the choice of a particular vector-symbolic architectures (see Table 2.1) is of little consequence to the behaviour of the model. This choice is at what we refer to as the sub-symbolic level (Chapter 2). At the symbolic level, we have to choose how these vectors are combined to create more complex representations (Chapter 4). The representation scheme used greatly influences the behaviour of the model. Unfortunately, there is a great deal of variability in the representations across models, indicating a lack of a consistent theory of representation. 
The HEM, HDM, BEAGLE, and DSHM models all use the same basic idea: given a set of co-occurring items represented as vectors, add to memory all combinations of them (all $n$ grams or open $n$-grams) or a random subset of all combinations to model degraded performance.

The vectors representing individual items are randomly generated by the models, but ought to be generated according to their semantic (as in Chapter 9) or perceptual features. Ultimately, to develop a proper theory representation, memory models need to be integrated with perceptual models.

4. Choice of learning rule In errorless learning, a vector representing an experience, $\mathbf{x}$, is simply added to memory when the model has that experience. In error-driven learning, the memory system is only updated when something surprising happens. That is to say, the memory makes a prediction $\mathbf{e}$ on the basis of prior experience $\mathbf{p}$, and memory is updated by the difference between the prediction and the experience. For example, when probed with $\mathbf{p}$, the MINERVAAL (Jamieson, Crump, \& Hannah, 2012) model retrieves echo e from memory, and is then updated with the vector $\mathbf{x}-\mathbf{e}$, the difference between expectation $\mathbf{e}$ and the actual experience $\mathbf{x}$.

5. Choice of knowledge organization When not using a placeholder vector, memory is updated as in the Holographic Exemplar Model (HEM; Jamieson \& Mewhort, 2011). In HEM, when an experience is added to memory, the experience is represented as a set of discrete items, which in turn are represented by vectors. An experience vector is constructed as a sum of the vectors representing individual items, pairs of items, triples, and so on. For example, the vector $\mathbf{x}$ 
for an experience composed of three unordered items represented by the vectors $\mathbf{a}, \mathbf{b}$, and $\mathbf{c}$ would be calculated as:

$$
\mathbf{x}=\mathbf{a}+\mathbf{b}+\mathbf{c}+\mathbf{a}^{*} \mathbf{b}+\mathbf{a}^{*} \mathbf{c}+\mathbf{b}^{*} \mathbf{c}+\mathbf{a}^{*} \mathbf{b}^{*} \mathbf{c}
$$

The order of the items can also be preserved by using the permutation $\mathbf{P}_{\text {before, }}$ as in Chapters 7, 8 and 9. Poor encoding can be simulated by a random sample of the individual items, pairs, triples, etc. rather than a complete set. The experience vector is then added to the memory system. When using a placeholder, the knowledge representation scheme is as described in Chapters 7, 8, and 9. Memory is updated once for each item that composes the experience. For example, given an experience composed of the items $a, b$, and $c$, represented by the environment vectors $\mathbf{e}_{\mathrm{a}}, \mathbf{e}_{\mathrm{b}}$, and $\mathbf{e}_{\mathrm{c}}$ respectively, to update memory with what we have learned about $a$ we would construct the cue vector $\mathbf{q}$ ?bc,

$$
\mathbf{q}_{? \mathrm{bc}}=\boldsymbol{\Phi}^{*} \mathbf{e}_{\mathrm{b}}+\boldsymbol{\Phi}^{*} \mathbf{e}_{\mathrm{c}}+\boldsymbol{\Phi}^{*} \mathbf{e}_{\mathrm{b}} * \mathbf{e}_{\mathrm{c}}
$$

where $\boldsymbol{\Phi}$ is the placeholder vector. In a many-to-many architecture, memory is updated by adding $\mathbf{q}_{\text {?bc }}$ to the memory vector for $a, \mathbf{m}_{\mathrm{a}}$. In a one-to-one or many-to-one architecture, memory $\mathbf{M}$ is updated by adding $\mathbf{e}_{\mathrm{a}} \mid \mathbf{q}$ ?bc where $\mid$ is vector concatenation. The environment vectors, in turn, can be compositional representations. For example, if the items are words, the environment vectors could represent orthography or phonology, as in Cox et al. (2011) or lower-level semantic representations, as in Chapter 9. If the items are sensory stimuli, the environment vectors could represent features of those stimuli, as in Kelly et al., (2013).

6. Choice of time variant process Why are people faster to remember somethings and slower to remember other things? Memory retrieval is a time variant process. But what is taking 
up all that time? There are three candidates in the literature: time to clean up the echo (Farrell \& Lewandowsky, 2002), sharpening the echo (Mewhort \& Johns, 2005), and sampling features from the probe (Cox \& Shiffrin, 2012). We prefer time to clean up the echo as it emerges naturally from a recurrent neural model of memory.

7. Choice of response decision criteria Cognitive models live and die by their ability to model and predict human experimental data. Much of the experimental data collected is reaction time data. How long does it take the model to recall something? How long does it take for the model to decide if a cue is familiar or unfamiliar? Reaction times in a memory model are a Gordian knot that tangles together participant strategy, the passage of time, and the quantity and quality of the information being retrieved from memory. By necessity, this aspect of memory models may remain ad hoc for quite some time.

In principle, integration into a larger cognitive system could help absolve memory models of their need for somewhat arbitrary criteria. ACT-R, unfortunately, treats time to retrieve information from memory as something that is purely internal to the memory model, rather than arising from the interaction between the memory retrieval process and an external decision process. The development of better cognitive architectures may eventually resolve this issue.

\subsection{Proposal for Future Models of Memory}

Here we summarize our preferred answers to each of the seven decisions in our framework, and thus outline what we feel to be the most fruitful direction for the development of future models:

(1) Given the demonstrations of equivalence made in this thesis, the choice of data structure is not a theoretical distinction but rather a pragmatic one. When implementing a neural 
model or a very large scale model, a many-to-one model is preferable. For small scale models, a one-to-one model is preferable. For large scale models, a many-to-many model is preferable.

(2) A memory model may constitute a hybrid of both hetero-associative and auto-associative representations and architectures.

(3) Vector-symbolic architectures (VSAs) such as holographic reduced representations provide a powerful representation scheme for memory models. However, while VSAs provide a means of combining vectors into arbitrarily complex representations, they do not provide a theory of how those vectors should be combined or how those vectors are generated. BEAGLE (Jones \& Mewhort, 2007) and HEM (Jamieson \& Mewhort, 2011) provide two different, though highly similar, theories of how vectors representing individual items should be combined to form memory traces. While the vectors are typically randomly generated for convenience, the vectors should be generated as outputs from a lower level memory model (as in Chapter 9) or as outputs from a perceptual system. Integration of memory models with perceptual models may be necessary if research is to move toward the development of a coherent theory of representation.

(4) We hypothesize that prediction error driven learning may be able to account for all learning and memory and so should be adopted in all future models until evidence to the contrary is found.

(5) Encoding knowledge as relative to concepts (concept-based) as in BEAGLE (Jones \& Mewhort, 2007) and HDM (Chapters 7 and 8) is an alternative to encoding structure within events (instance-based) that should be explored in more models. It is an open 
research question whether or not concept-based knowledge structures can emerge from instance-based knowledge structures or if both types of knowledge are necessary.

(6) We prefer time to clean up the echo as the main process that accounts for variations in human response times for memory tasks.

(7) We hold that response decision criteria are an artifact of modelling memory separate from other cognitive functions. Response times are emergent from the interactions of memory with perception, action, and the participant's meta-cognitive strategy for the experimental task, and, as such, are not easy to model. Better integration of memory models with cognitive architectures is necessary if researchers are to move away from ad hoc criterion setting.

\subsection{Conclusion}

We conduct a comparative analysis of the matching strengths of hetero-associative and autoassociative models as well as concept-based and instance-based models. We find that MINERVA 2 cannot account for the fan effect task discussed in Chapter 7 and that this may be indicative of a deep flaw in the MINERVA 2 model, though it is a flaw that could be remedied in a number of ways. We propose a theoretical framework for current and future models of memory. The framework consists of Hintzman's key assumptions and a set of seven key decisions. On the basis of this framework, we sketch a course of development for future memory models. 


\section{Chapter 11 Conclusion}

Imagine that memory is a cardboard box filled to the brim with things that are important to you. In fact, imagine that it has Important stuff! scribbled on the side of it in black marker, triple underlined. But at any particular moment, only one thing in the box is important to you: the thing you need right now. How do you find it?

Some early models of memory suggested that finding something in memory was a serial search process (e.g., Tulving, 1976). When you need to find something in the box, you go through the contents of the box one item at a time until you find the thing you need. If the thing you are looking for is not in the box, you will have to search the entire box to find that out. However, in recognition tasks, people make recognition judgements very quickly. In particular, people make judgements that a test item wasn't in the study set too quickly for an exhaustive serial search model to be correct (Atkinson \& Juola, 1974).

Other early models of memory suggested that items in memory can be accessed directly (e.g., Kintsch, 1970). If the things in the box are carefully organized, you can just grab the item you need without searching. However, direct access models have trouble accounting for the effects of other items on the recognition of a given item (see Clark \& Gronlund, 1996, for a review). That said, ACT-R DM (Anderson \& Lebiere, 1999) is a direct access model that is able to account for such effects in recognition tasks, such as the fan effect task (Anderson \& Reder, 1999).

In global matching models, what memory retrieves is an aggregate across all experiences. To find something in the box, you turn the box upside down, dump its contents on the floor, and 
then rifle through the jumbled mess to try to find the thing you need. Global matching models are able to account for the speed of retrieval, like direct access models, but also the influence of other items on retrieval, like serial search models.

Mathematical analysis of the global matching models (Clark \& Gronlund, 1996; Hintzman, 1990; Humphreys et al., 1989b; Kahana et al., 2005), including MINERVA 2 (Hintzman, 1984), CHARM (Eich, 1982), TODAM (Murdock, 1993), the Matrix model (Humphreys et al., 1989b), and SAM (Gillund \& Shiffrin, 1984) found strong similarities between the models. However, global matching models that stored all memory as a composite were viewed as a different class from global matching models that stored memories as separate vectors (Clark \& Gronlund, 1996; Hintzman, 1990).

Building on those earlier analyses of memory models, we classify current vector-based memory models into three categories: many-to-one, one-to-one, and many-to-many (Chapter 4). In many-to-one models, many experiences are summed together to be stored as one composite, such that all items are represented as distributed across a shared set of units. In one-to-one models, there is a one-to-one relationship between the number of experiences the model has had and the the number of vectors stored in memory. In many-to-many models, each experience is represented across many memory vectors, and each memory vector represents many experiences. Many-to-one models include Hebbian neural networks, tensor models that generalize Hebbian learning from associations between pairs to associations between arbitrarily many items, and vector-symbolic architectures, such as a holographic vectors, that compress tensors into vectors of fixed dimensionality. Vector-symbolic architectures provide a powerful formalism 
for representing knowledge in memory models that confers advantages over both conventional symbolic models, such as ACT-R, and conventional neural network models (Chapters 2 and 3).

Many-to-one models can be implemented as neural models and, because they are invariant in size with respect to the number of experiences, can be scaled to arbitrarily long-term learning. Conversely, it is not clear that this is the case for many-to-many and one-to-one models. With an interest in addressing the question of the validity of many-to-many and one-to-one models, as well as with an interest of moving toward theoretical unification, we conduct an analysis of these three classes of model.

We find that these three classes of models are not different in kind (Chapter 5). In particular, we prove that the one-to-one model MINERVA 2 (Hintzman, 1984) is exactly mathematically equivalent to a many-to-one composite model, an auto-associative memory implemented as a fourth-order tensor. MINERVA 2 is also approximately equivalent to a fourthorder tensor compressed into a holographic vector. Additionally, many-to-many models such as BEAGLE (Jones \& Mewhort, 2007) or HDM can be understood as collections of the echoes retrieved from a one-to-one or many-to-one model. Thus, these three classes of model can be understood as different computational descriptions of the same underlying theoretical framework. As such, many-to-many and one-to-one models can be scaled up to arbitrarily longterm learning and implemented as a biologically realistic neural model (Chapters 5 and 6).

With the goal of advancing toward a unified theory of human memory, we propose a theoretical framework for characterizing computational memory models in terms of the key assumptions of global matching models, as set out by Hintzman (1986), and a set of seven critical decisions of theory and implementation (Chapter 10). These seven decisions are: (1) 
choice of data structure (many-to-one, one-to-one, many-to-many), (2) choice of hetero- or autoassociative architecture, (3) choice of representation scheme, (4) choice of learning rule, (5) choice of knowledge organization, (6) choice of time variant process, and (7) choice of response decision criteria.

The theoretical framework we propose allows for memory to be modelled at multiple levels of analysis, from the level of individual, biological neurons (Chapters 5 and 6), to the level of messages passed between groups of those neurons (many-to-one models), to the level of the events processed by memory (one-to-one models), to the level of the concepts which emerge as aggregates across those events (many-to-many models), and on up, to the arbitrarily abstract concepts that emerge from aggregating across concepts (Chapter 9). Furthermore, we find that estimates of Bayesian probability arise from the vector algebra of the model (Chapter 7), such models based on Bayesian probability, such as ACT-R DM (Anderson \& Reder, 1999), can be understood as higher-level descriptions of our vector-based theoretical framework.

With the aim of advancing toward a unified theory of cognition as a whole, we demonstrate integration of a model within this framework, HDM, into the commonly used cognitive architecture, ACT-R (Chapter 7). We demonstrate that HDM can account for tasks performed by both ACT-R's declarative memory system (Chapter 7) and ACTR's procedural memory system (Chapter 8).

Simon (1969) famously argued that to account for the complexity of human behaviour we need to account for the influence of two environments: the external environment of the world and the internal environment of memory. We aim to move towards an account of memory that can be implemented as a realistic neural model, can account for diverse experimental memory 
phenomena, can detect arbitrarily abstract associations in its environment, can be scaled up to arbitrarily long-term learning, and can be integrated into a cognitive architecture. Thus we feel that the memory framework we propose is an important step towards a working model of the human mind.

\subsection{Overview of Contributions}

\section{Chapter 1 Introduction}

-We present a taxonomic classification of memory models.

\section{Chapter 2 Vectors to Symbols: The Sub-Symbolic Level}

-We characterize computational models of cognition in terms of two levels: symbolic and sub-symbolic. Design decisions are made at both levels.

- We provide an introduction to vector-symbolic architectures (VSAs), which provide a sub-symbolic scheme for knowledge representation.

\section{Chapter 3 Addressing Criticisms of Connectionist and Symbolic Models}

-We review the arguments in the literature against traditional connectionist (neural network) or symbolic models of cognition and in favour of vector-symbolic architectures. VSAs combine the qualities of connectionist and symbolic models.

-We note that whether VSAs can be applied to problem domains that cannot be trivially discretized into symbols remains an open question.

\section{Chapter 4 Symbols to Cognition: The Symbolic Level}

-We characterize the symbolic level of VSAs in terms of cognitive processes, in particular how information is represented, stored, and retrieved. 
-We classify memory models in the literature as many-to-one, one-to-one, and many-tomany according to their implementation of these processes.

- On the basis of our analysis, we suggest means for theoretical unification of existent models.

\section{Chapter 5 The Memory Tesseract}

- We conduct a wide review and comparative analysis of vector-based computational memory models in the literature.

-We show that "local match" and "global match" recognition models (Clark \& Gronlund, 1996) differ only in the exponent used to weight the dot products of the memory traces with the probe.

-We prove exact mathematical equivalence between a one-to-one model, MINERA 2 (Hintzman, 1984), and a many-to-one model, an auto-associative composite memory implemented as a fourth order tensor, or memory tesseract.

- We additionally prove that MINERVA 2 is approximately equivalent to an autoassociative fourth order tensor compressed into a holographic vector.

-We outline a neural model of MINERVA 2 as holographic vector. The neural model is a set of parallel recurrent neural circuits constructed out of biologically realistic simulated neurons using the Neural Engineering Framework (Eliasmith, 2013).

- We show that the memory vectors of a many-to-many model, BEAGLE (Jones \& Mewhort, 2007), approximate the echoes of a MINERVA model. 
- Thus, we demonstrate that many-to-one, one-to-one, and many-to-many models can all be understood as instantiations of the same theoretical framework, and one-to-one and many-to-many can be implemented as many-to-one models.

- Thus, we demonstrate that one-to-one, and many-to-many models can be plausibly implemented in neurons.

- Given that many-to-one models can be scaled to arbitrarily long-term tasks due to the fact that they are invariant in scale with respect to the number of traces stored, we have thus demonstrated that one-to-one and many-to-many can also be scaled to arbitrarily long-term tasks re-implemented in a many-to-one architecture.

\section{Chapter 6 Proof of Concept Neural Implementation of MINERVA}

- We demonstrate that MINERVA 2, understood as a fourth order tensor, can be implemented as a recurrent neural circuit constructed out of biologically realistic simulated neurons using the Neural Engineering Framework (Eliasmith, 2013).

\section{Chapter 7 The Fan Effect and ACT-R}

-We present Holographic Declarative Memory (HDM), a many-to-many model of memory based on DSHM (Rutledge-Taylor et al., 2014) and BEAGLE (Jones \& Mewhort, 2007).

- Holographic Declarative Memory is integrated into the ACT-R (Anderson \& Lebiere, 1999) cognitive architecture as a module.

- We use HDM to model the fan effect task (Anderson, 1974), and without adjusting any parameters, we achieve a fit to human performance comparable to ACT-R's declarative memory module (DM). 
-We analyze the ACT-R DM model of the fan effect task (Anderson \& Reder, 1999) and determine that ACT-R DM's performance is a function of the product of the fans with slope, power, and intercept determined by fitting parameters.

- We analyze the HDM model of the task and determine that the vector algebra of HDM estimates the Bayesian probability that an item in memory is relevant given the probe.

- Thus, HDM is an implementation of Anderson's (1991) rational analysis, is closely related to quantum models of human probability judgement (Busemeyer et al., 2011) and can be understood as a lower level description of the associative mechanism in ACT-R DM, which uses Bayesian probability to compute association strengths.

\section{Chapter 8 Procedural Learning and Decision Making}

- We demonstrate that HDM can model human performance in a procedural memory and decision making task, specifically the task described by Walsh and Anderson (2011).

- Thus, we propose that HDM can be used as a substitute for both ACT-R's declarative memory and procedural memory systems.

\section{Chapter 9 Memory for Abstract Associations}

- We define orders of association as the degree of separation between concepts in memory or words in language.

- We propose orders of association as a tool for the analysis of the structures of natural language.

-We develop the Hierarchical Holographic Model, a model of memory that is sensitive to arbitrarily high orders of association. The model consists of a series of BEAGLE / 
DHSM / HDM models, where each higher layer uses the concepts constructed by the lower layer as its basic units.

- We demonstrate performance of the model on artificial data and a small corpus of 15 children's novels.

-We find that the higher layers of the model are able to exploit higher-order associations to gain additional semantic and syntactic information.

\section{Chapter 10 Memory Model Framework}

-We conduct a comparative analysis of hetero-associative (e.g., TODAM; Murdock, 1993) and auto-associative memory models (e.g., MINERVA).

- We conduct a comparative analysis of concept-based (e.g., HDM) and instance-based memory models (e.g., MINERVA).

- We compare performance of MINERVA and HDM on the fan effect task (Anderson, 1974) and find that MINERVA 2 does not predict the fan effect.

-We demonstrate HDM can be re-implemented as a MINERVA model with no effect to HDM's behavioural predictions on the fan effect task.

- We demonstrate that a MINERVA 2 model that uses two probes (as HDM does) replicates the fan effect.

-We analyze the mathematics of MINERVA 2's vector algebra to determine why MINERVA 2 does not predict the fan effect.

- On the basis of this analysis, we suggest that the failure of MINERVA 2 on the fan effect is indicative of a wider problem with the MINERVA 2 model. 
- We suggest that MINERVA 2 may be able to predict the fan effect if made sensitive to the strength / variance of the weighted means retrieved from memory in the echo.

- We discuss the difficulties of generating response decisions and response times from memory models in a theoretically justified manner.

- On the basis of our comparative analysis of memory models, we present a framework for computational models of memory, consisting of a set of common assumptions and seven decisions.

-Within this framework, we outline the space of what we believe will be the most fruitful approaches to developing future computational models of human memory. 


\section{References}

Anderson, J. A. (1973). A theory for the recognition of items from short memorized lists. Psychological Review, 80, 417-438. doi: 10.1037/h0035486

Anderson, J. R. (1974). Retrieval of propositional information from long-term memory. Cognitive Psychology, 6, 451-474. doi: 10.1016/0010-0285(74)90021-8

Anderson, J. R. (1991). The place of cognitive architectures in a rational analysis. In K. Van Len (Ed.), Architectures for Intelligence. Hillsdale, NJ: Erlbaum.

Anderson, J. R. \& Lebiere, C. (1998). The Atomic Components of Thought. Mahwah, NJ: Lawrence Erlbaum Associates.

Anderson, J. R. \& Reder, L. M. (1999). The fan effect: New results and new theories. Journal of Experimental Psychology: General, 128, 186-197. doi=10.1.1.139.8243

Atkinson, R. C., \& Juola, J. F. (1974). Search and decision processes in recognition memory. In D. H. Krantz, R. C. Atkinson, R. D. Luce, \& P. Suppes (Eds.), Contemporary developments in mathematical psychology: Vol. 1. Learning, memory, and thinking (pp. 243-293). San Francisco: W. H. Freeman.

Brooks, R. A. (1991). Intelligence without representation. Artificial Intelligence, 47, 139-159.

Burgess, C., \& Lund, K. (1997). Modelling parsing constraints with high-dimensional context space. Language and Cognitive Processes, 12, 177-210.

Busemeyer, J. R., Pothos, E. M., Franco, R., \& Trueblood, J. (2011). A quantum theoretical explanation for probability judgement errors. Psychological Review, 118, 193-218. doi: $10.1037 / \mathrm{a} 0022542$ 
Chomsky, N. (1956). Three Models for the Description of Language. IRE Transactions on Information Theory 2, 113-124. doi:10.1109/TIT.1956.1056813

Christodoulopoulos, C., Goldwater, S., \& Steedman, M. (2010). Two decades of unsupervised POS induction: How far have we come? In Proceedings of the 2010 Conference on Empirical Methods in Natural Language Processing (pp. 575-584). Association for Computational Linguistics.

Chubala, C. M., Johns, B. T., Jamieson, R. K., \& Mewhort, D. J. K. (2016). Applying an exemplar model to an implicit rule-learning task: Implicit learning of semantic structure. The Quarterly Journal of Experimental Psychology, 69, 1049-1055. doi: $10.1080 / 17470218.2015 .1130068$

Clark, A. (2001). Symbol systems (Chapter 2). Mindware: An Introduction to the Philosophy of Cognitive Science, 27-38. Oxford University Press.

Clark S. E. \& Gronlund S. D. (1996). Global matching models of recognition memory: How the models match the data. Psychonomic Bulletin \& Review, 3, 37-60. doi: 10.3758/ BF03210740

Cox, G. E., Kachergis, G., Recchia, G., \& Jones, M. N. (2011). Towards a Scalable Holographic Representation of Word Form. Behavior Research Methods, 43, 602-615. doi: 10.3758/ s13428-011-0125-5

Cox, G. E., \& Shiffrin, R. M. (2012). Criterion setting and the dynamics of recognition memory. Topics in Cognitive Science, 4, 135-150. doi: 10.1111/j.1756-8765.2011.01177.x 
Dougherty, M. R. P., Gettys, C. F., \& Ogden, E. E. (1999). MINERVA-DM: A memory processes model for judgments of likelihood. Psychological Review, 106, 180-209. doi: 10.1037/0033-295X.106.1.180

Eich, J. M. (1982). A composite holographic associative recall model. Psychological Review, 89, 627-661. doi: 10.1037/0096-3445.119.2.145

Eliasmith, C. (2013). How to build a brain: A neural architecture for biological cognition. Oxford University Press, New York, NY.

Eliasmith, C. (n.d.). Function quality and number of neurons. Retrieved from $<\underline{\mathrm{http}: / /}$ nbviewer.ipython.org/github/ctn-archive/technical-reports/blob/master/Number-ofneurons-for-given-accuracy-of-any-function.ipynb>

Eliasmith, C., Stewart T. C., Choo X., Bekolay T., DeWolf T., Tang Y., Rasmussen, D. (2012). A large-scale model of the functioning brain. Science, 338, 1202-1205. doi: 10.1126/ science. 1225266

Eliasmith, C., \& Thagard, P. (2001). Integrating structure and meaning: a distributed model of analogical mapping. Cognitive Science, 25, 245-286. doi: 10.1016/ S0364-0213(01)00036-2

Farrell, S. \& Lewandowsky, S. (2002). An endogenous distributed model of ordering in serial recall. Psychonomic Bulletin \& Review, 9, 59-79. doi: 10.3758/BF03196257

Fass, C., Ginelli, M. \& Turtle, B. (1996). Six Degrees of Kevin Bacon. Plume Books.

Firth, J. R. (1957). A synopsis of linguistic theory. Studies in linguistic analysis. Oxford: Blackwell. 
Fodor, J. A., \& Pylyshyn, Z. W. (1988). Connectionism and cognitive architecture: a critical analysis. Cognition, 28, 3-71.

Franklin, D. R. J., \& Mewhort, D. J. K. (2015). Memory as a hologram: An analysis of learning and recall. Canadian Journal of Experimental Psychology, 69, 115-135. doi: 10.1037/ cep0000035

Fredrickson, B. L. (2001). The role of positive emotions in positive psychology: The broadenand-build theory of positive psychology. American Psychologist, 56, 218-226. doi: 10.1037/0003-066X.56.3.218

Furber, S. B., Galluppi, F., Temple, S., \& Plana, L. A. (2014). The spinnaker project. Proceedings of the IEEE, 102, 652-665. doi: 10.1109/JPROC.2014.2304638

Gabor, D. (1969). Associative holographic memories. IBM Journal of Research and Development, 13, 156-159. doi: 10.1147/rd.132.0156

Gayler, R. W. (2003) Vector symbolic architectures answer Jackendoff's challenges for cognitive neuroscience. In P. Slezak (Ed.), Proceedings of the Joint International Conference on Cognitive Science (pp. 133-138). Sydney, Australia: University of New South Wales.

Gayler, R. W. \& Levy, S. D. (2009). A distributed basis for analogical mapping. New frontiers in analogy research; Proceedings of the Second International Analogy Conference Analogy 09, 165-174.

Gillund, G., \& Shiffrin R. M. (1984). A retrieval model of both recognition and recall. Psychological Review, 91, 1-67.

Goffman, Casper (1969). And what is your Erdős number? American Mathematical Monthly, 76, 791. doi:10.2307/2317868 
Goldinger, S. D. (1998). Echoes of echoes? An episodic theory of lexical access. Psychological Review, 105, 251-279. doi: 10.1037/0033-295X.105.2.251

Griffiths, T. L., Steyvers, M., \& Tenenbaum, J. B. (2007) Topics in semantic representation. Psychological Review, 114, 211-244. doi: 10.1037/0033-295X.114.2.211

Hannagan, T., Dupoux, E., \& Christophe, A. (2011). Holographic string encoding. Cognitive Science, 35, 79-118. doi: 10.1111/j.1551-6709.2010.01149.x

Hinton, G. E. (1990). Mapping part-whole hierarchies into connec- tionist networks. Artificial Intelligence, 46, 47-76.

Hintzman, D. L. (1984). MINERVA 2: A simulation model of human memory. Behavior Research Methods, Instruments, and Computers, 16, 96-101. doi: 10.3758/BF03202365

Hintzman, D. L. (1986). "Schema abstraction" in multiple-trace memory models. Psychological Review, 93, 411-428. doi: 10.1037/0033-295X.95.4.528

Hintzman, D. L. (1988). Judgments of frequency and recognition memory in a multiple-trace memory model. Psychological Review, 95, 528-551. doi: 10.1037/0033-295X.95.4.528

Hintzman, D. L. (1990). Human learning and memory: Connections and dissociations. Annual Review of Psychology, 41, 109-139. doi: 10.1146/annurev.ps.41.020190.000545

Hintzman, D. L. (2016). Is memory organized by temporal contiguity? Memory \& cognition, 44, 365-375. doi: 10.3758/s13421-015-0573-8

Howard, M. W., \& Kahana, M. J. (2002). A distributed representation of temporal context. Journal of Mathematical Psychology, 46, 269-299. doi: 10.1006/jmps.2001.1388 
Humphreys, M. S., Bain, J. D., \& Pike, R. (1989a). Different ways to cue a coherent memory system: A theory for episodic, semantic, and procedural tasks. Psychological Review, 96, 208-233. doi: 10.1037/0033-295X.96.2.208

Humphreys, M. S., Pike, R., Bain, J. D., \& Tehan, G. (1989b). Global matching: A comparison of the SAM, Minerva II, Matrix, and TODAM models. Journal of Mathematical Psychology, 33, 36-67. doi: 10.1016/0022-2496(89)90003-5

Jackendoff, R. (2002). Foundations of language: Brain, meaning, grammar, evolution. Oxford University Press.

Jamieson, R. K., Crump, M. J. C., \& Hannah, S. D. (2012). An instance theory of associative learning. Learning \& Behavior, 40, 61-82.

Jamieson, R. K., \& Mewhort, D. J. K. (2009). Applying an exemplar model to the artificialgrammar task: Inferring grammaticality from similarity. The Quarterly Journal of Experimental Psychology, 62, 550-575. doi: 10.1080/17470210802055749

Jamieson, R. K., \& Mewhort, D. J. K. (2011). Grammaticality is inferred from global similarity: A reply to kinder (2010). The Quarterly Journal of Experimental Psychology, 64, 209-216.

Jamieson, R. K., Mewhort, D. J. K., \& Hockley, W. E. (2016). A computational account of the production effect: Still playing twenty questions with nature. Canadian Journal of Experimental Psychology, 70, 154-164. doi: 10.1037/cep0000081

Johns, B. T., Jamieson, R. K., Crump, M. J. C., Jones, M. N., \& Mewhort, D. J. K. (in press). The combinatorial power of experience. In Proceedings of the 38th Annual Meeting of the Cognitive Science Society. Austin, TX: Cognitive Science Society. 
Johns, B. T., Jones, M. N., \& Mewhort, D. J. K. (2012). A synchronization account of false recognition. Cognitive psychology, 65, 486-518. doi: 10.1016/j.cogpsych.2012.07.002

Jones, M. N., Kintsch, W., \& Mewhort, D. J. K. (2006). High-dimensional semantic space accounts of priming. Journal of Memory and Language, 55, 534-552. doi: 10.1016/j.jml. 2006.07.003

Jones, M. N., \& Mewhort, D. J. K. (2007). Representing word meaning and order information in a composite holographic lexicon. Psychological Review, 114, 1-37. doi:

10.1037/0033-295X.114.1.1

Kahana, M. J., Rizzuto, D. S., \& Schneider, A. R. (2005). Theoretical correlations and measured correlations: Relating recognition and recall in four distributed memory models. Journal of Experimental Psychology: Learning, Memory, and Cognition, 31, 933-953. doi: 10.1037/0278-7393.31.5.933

Kanerva, P. (1996). Binary spatter-coding of ordered k-tuples. Proceedings of the 1996 International Conference on Artificial Neural Networks, 869-873.

Kelly, M. A. (2010). Advancing the theory and utility of holographic reduced representations (Master's thesis, Queen's University, Canada). ProQuest Dissertations and Theses, retrieved from http://search.proquest.com/docview/853293422

Kelly, M. A., Blostein, D., \& Mewhort, D. J. K. (2013). Encoding structure in holographic reduced representations. Canadian Journal of Experimental Psychology, 67, 79-93. doi: $10.1037 / \mathrm{a} 0030301$

Kelly, M. A., Kwok, K., West, R. L. (2015). Holographic declarative memory and the fan effect: A test case for a new memory model for ACT-R. In N. A. Taatgen, M. K. van Vugt, J. P. 
Borst, \& K. Mehlhorn (Eds.), Proceedings of the 13th International Conference on Cognitive Modeling (pp. 148-153). Groningen, the Netherlands: University of Groningen.

Kelly, M. A., Mewhort, D. J. K., \& West, R. L. (2014). The memory tesseract: Distributed MINERVA and the unification of memory. In Bello, P., Guarini, M., McShane, M., \& Scassellati, B. (Eds.), Proceedings of the 36th Annual Conference of the Cognitive Science Society (pp. 2483-2488). Austin TX: Cognitive Science Society. https:// mindmodeling.org/cogsci2014/papers/431/

Kelly, M. A. \& West, R. L. (2012). From vectors to symbols to cognition: The symbolic and subsymbolic aspects of vector-symbolic cognitive models. In N. Miyake, D. Peebles, \& R. P. Cooper (Eds.), Proceedings of the 34th Annual Conference of the Cognitive Science Society (pp. 1768-1773). Austin, TX: Cognitive Science Society. http:// mindmodeling.org/cogsci2012/papers/0311/

Kelly, M. A. \& West, R. L. (2013). Decision-making in a dynamically structured holographic memory: Learning from delayed feedback. In R. West \& T. Stewart (Eds.), Proceedings of the 12th International Conference on Cognitive Modeling (pp. 47-52). Ottawa, Canada: Carleton University. http://iccm-conference.org/2013-proceedings/papers/0008/ index.html

Kintsch, W. (1970). Models for free recall and recognition. In D. A. Norman (Ed.), Models of human memory. New York: Academic Press.

Knight, J., Voelker, A. R., Mundy, A., Eliasmith, C. \& Furber, S. (in press). Efficient spinnaker simulation of a heteroassociative memory using the neural engineering framework. In The 2016 International Joint Conference on Neural Networks (IJCNN). IEEE. 
Kwantes, P. J. (2005). Using context to build semantics. Psychonomic Bulletin \& Review, 12, 703-710.

Kwantes, P. J., \& Mewhort, D. J. K. (1999). Modeling lexical decision and word naming as a retrieval process. Canadian Journal of Experimental Psychology, 53, 306-315.

Kwok, K. (2016). Complex fan analysis, an extension of ACT-R fan effect model (Ph.D. thesis, Carleton University, Canada). MacOdrum Library Catalogue, retrieved from http:// catalogue.library.carleton.ca/record=b3859381

Kwok, K., West, R. L., \& Kelly, M. (2015). The fan effect in overlapping data sets and logical inference. In D. C. Noelle, R. Dale, A. S. Warlaumont, J. Yoshimi, T. Matlock, C. D. Jennings, \& P. P. Maglio (Eds.), Proceedings of the 37th Annual Conference of the Cognitive Science Society (pp. 1231-1236). Austin TX: Cognitive Science Society.

Laird, J. E. (2012). The Soar Cognitive Architecture. MIT Press, Cambridge, MA.

Lakatos, I. (1976). Falsification and the methodology of scientific research programmes. In Can Theories be Refuted? (pp. 205-259). Springer Netherlands.

Landauer, T. K., \& Dumais, S. T. (1997). A solution to Plato's problem: The latent semantic analysis theory of acquisition, induction, and representation of knowledge. Psychological Review, 104, 211-240.

Langley, P., Laird, J. E., \& Rogers, S. (2009). Cognitive architectures: Research issues and challenges. Cognitive Systems Research, 10, 141-160.

Lebiere, C., Gonzalez, C. \& Martin, M.K. (2007). Instance-based decision making model of repeated binary choice. In Lewis, Polk, \& Laird (Eds.), Proceedings of the 8th International Conference on Cognitive Modeling (pp. 77-82). Ann Arbor, MI. 
Levy, S.D., \& Gayler, R.W. (2009). "Lateral inhibition" in a fully distributed connectionist architecture. In A. Howes, D. Peebles, \& R. Cooper (Eds.), Proceedings of the 9th International Conference on Cognitive Modeling - ICCM 2009 (pp.318-323). Manchester, UK.

Lewandowsky, S. \& Farrell, S. (2008). Short-term memory: New data and a model. In B. H. Ross (Ed.), The psychology of learning and motivation, 49, 1-48. doi: 10.1016/ S0079-7421(08)00001-7

Locke, J. (1690) An essay concerning human understanding.

Longuet-Higgins, H. C. (1968). Holographic Model of Temporal Recall. Nature, 217, 104.doi: $10.1038 / 217104 \mathrm{a} 0$

Mewhort, D. J. K., Johns, E. E. (2000). The extralist-feature effect: Evidence against item matching in short-term recognition memory. Journal of Experimental Psychology: General, 129, 262-284. doi: 10.1037/0096-3445.129.2.262

Mewhort, D. J. K., \& Johns, E. E. (2005). Sharpening the echo: An iterative-resonance model for short-term recognition memory. Memory, 13, 300-307.

Minsky, M. L., \& Papert, S. A. (1969). Perceptrons. Cambridge, MA: MIT Press.

Minton, S. N. (1990). Quantitative results concerning the utility of explanation-based learning. Artificial Intelligence, 42, 363-391.

Murdock, B. B. (1982). A theory for the storage and retrieval of item and associative information. Psychological Review, 89, 609-626. doi: 10.1037/0033-295X.89.6.609 
Murdock, B. B. (1989). Learning in a distributed memory model. In C. Izawa (Ed.), Current Issues in Cognitive Processes: The Tulane Symposium of Cognition. Hillsdale, NJ: Erlbaum.

Murdock, B. B. (1993). TODAM2: a model for the storage and retrieval of item, associative and serial-order information. Psychological Review, 100, 183-203. doi: 10.1037/0033-295X. 100.2.183

Newell, A. (1973). You can't play 20 questions with nature and win. In W. G. Chase (Ed.), Visual information processing. New York: Academic Press.

Nosofsky, R. M. (1986). Attention, similarity and the identification-categorization relationship. Journal of Experimental psychology: General, 115, 39-57. doi:

10.1037/0096-3445.115.1.39

Nosofsky, R. M. (1991). Tests of an exemplar model for relating perceptual classification and recognition memory. Journal of Experimental Psychology: Human Perception and Performance, 17, 3-27. doi: 10.1037/0096-1523.17.1.3

Osth, A. F., \& Dennis, S. (2015). Sources of interference in item and associative recognition memory. Psychological Review, 122, 260-311. doi: 10.1037/a0038692

Plate, T. A. (1995). Holographic reduced representations. IEEE Transactions on Neural Networks, 6, 623-641. doi: 10.1109/72.377968

Plate, T. A. (2000). Analogy retrieval and processing with distributed vector representations. Expert Systems: The International Journal of Knowledge Engineering and Neural Networks, 17, 29-40. doi: 10.1111/1468-0394.00125 
Plate, T. A. (2003). Holographic Reduced Representations. CSLI Lecture Notes Number 150, CSLI Publications, Stanford, CA.

Popper, K. R. (1959). The logic of scientific discovery. London: Hutchinson.

Pylyshyn, Z. (1978). Computational models and empirical constraints. Behavioral and Brain Sciences, 1, 91-99 doi: 10.1017/S0140525X00059793

Pylyshyn, Z. (1989). Computing in Cognitive Science. In Posner, M. (Ed.) Foundations of Cognitive Science. Cambridge: MIT Press.

Raaijmakers, J. G. W., \& Shiffrin, R. M. (1981). Search of associative memory. Psychological Review, 88, 93-134. doi: 10.1037/0033-295X.88.2.93

Rasmussen, D., \& Eliasmith, C. (2011). A neural model of rule generation in inductive reasoning. Topics in Cognitive Science, 3, 140-153. doi: 10.1111/j.1756-8765.2010.01127.x

Rutledge-Taylor, M. F., Kelly M. A., West, R. L., \& Pyke, A. A. (2014). Dynamically structured holographic memory. Biologically Inspired Cognitive Architectures, 9, 9-32. doi: 10.1016/j.bica.2014.06.001

Rutledge-Taylor, M. F., Pyke, A. A., West, R. L., Lang, H. (2010). Modeling a three term fan effect. In D. D. Salvucci \& G. Gunzelmann (Eds.), Proceedings of the 10th International Conference on Cognitive Modeling (pp. 211-216). Philadelphia, PA: Drexel University.

Rutledge-Taylor, M. F., Vellino, A., \& West, R. L. (2008). A holographic associative memory recommender system. In Proceedings of the 3rd International Conference on Digital Information Management (pp. 87-92). London, UK.

Rutledge-Taylor, M. F. \& West, R. L. (2007). MALTA: Enhancing ACT-R with a holographic persistent knowledge store. In D. S. McNamara \& J. G. Trafton (Eds.), Proceedings of the 
29th Annual Conference of the Cognitive Science Society. 1433-1438. Nashville, TN:

Cognitive Science Society.

Sahlgren, M., Holst, A., \& Kanerva, P. (2008). Permutations as a means to encode order in word space. Proceedings of the 30th Annual Conference of the Cognitive Science Society, $1300-1305$.

Saussure, F. (1916). Cours de linguistique générale. C. Bally, A. Sechehaye, \& A. Riedlinger, (Eds.). Lausanne, France: Payot.

Shiffrin, R. M., Ratcliff, R., \& Clark, S. E. (1990). List-strength effect: II. Theoretical mechanisms. Journal of Experimental Psychology: Learning, Memory, and Cognition, 16, 179-195. doi: 10.1037/0278-7393.16.2.179

Shiffrin, R. M., \& Steyvers, M. (1997). A model for recognition memory: REM-retrieving effectively from memory. Psychonomic Bulletin \& Review, 4, 145-166. doi: 10.3758/ BF03209391

Simon, H. A. (1969). The psychology of thinking: Embedding artifice in nature (Chapter 2). In Sciences of the artificial (pp. 23-26). MIT Press.

Smolensky, P. (1990). Tensor product variable binding and the representation of symbolic structures in connectionist systems. Artificial Intelligence, 46, 159-216. doi: $10.1016 / 0004-3702(90) 90007-\mathrm{M}$

Stewart, T. C., Bekolay, T., and Eliasmith, C. (2012) Learning to select actions with spiking neurons in the basal ganglia. Frontiers in Neuroscience, 6:2, 1-14.

Stewart, T. C., \& Eliasmith, C. (2008). Building Production Systems with Realistic Spiking Neurons. In B. C. Love, K. McRae, \& V. M. Sloutsky (Eds.), Proceedings of the 30th 
Annual Meeting of the Cognitive Science Society (pp. 1759-1764). Austin, Texas:

Cognitive Science Society.

Thagard, P., \& Stewart, T. C. (2011). The Aha! experience: Creativity through emergent binding in neural networks. Cognitive Science, 35, 1-33. doi: 10.1111/j.1551-6709.2010.01142.x

Thomas, R. P., Dougherty, M. R., Sprenger, A. M., \& Harbison, J. I. (2008). Diagnostic hypothesis generation and human judgment. Psychological Review, 115, 155-185.

Tulving, E. (1976). Ecphoric processes in recall and recognition. In J. Brown (Ed.), Recall and recognition. London: Wiley.

Tulving, E., \& Watkins, M.J. (1973). Continuity between recall and recognition. American Journal of Psychology, 86, 739-748.

Walsh, M. M., \& Anderson, J. R. (2011). Learning from delayed feedback: Neural responses in temporal credit assignment. Cognitive, Affective, and Behavioral Neuroscience, 11, 131-143. doi: 10.3758/s13415-011-0027-0

West, R. L., Pyke, A. A., Rutledge-Taylor, M. F., \& Lang, H. (2010). Interference and ACT-R:

New evidence from the fan effect. In D. D. Salvucci \& G. Gunzelmann (Eds.), Proceedings of the 10th International Conference on Cognitive Modeling (pp. 277-281). Philadelphia, PA: Drexel University.

Wittgenstein, L. (1958). Philosophical investigations (G. E. M., Anscombe, Trans.). Oxford: Blackwell. 